\title{
SURFACE MICROMACHINED MEMS VARIABLE CAPACITOR WITH TWO- CAVITY FOR ENERGY HARVESTING
}

A Dissertation

Presented to

The Faculty of the Graduate School

At the University of Missouri

In Partial Fulfillment

of the Requirements for the Degree

Doctor of Philosophy

by

JIE LIN

Dr. Mahmoud Almasri, Dissertation Supervisor

December, 2013 
The undersigned, appointed by the dean of the Graduate School, have examined the dissertation entitled

SURFACE MICROMACHINED MEMS VARIABLE CAPACITOR WITH TWOCAVITY FOR ENERGY HARVESTING

Presented by Jie Lin

A candidate for the degree of

Doctor of Philosophy

And hereby certify that, in their opinion, it is worthy of acceptance.

Mahmoud Almasri

Zaichun Feng

Satish Nair

Robert O'Connell 


\section{ACKNOWLEDGEMENTS}

I would like to express my appreciation to my PhD advisor, Dr. Mahmoud Almasri, for giving me the opportunity to work with him, for funding my $\mathrm{PhD}$ study and for providing me with the continuous guidance, constructive feedback and encouragement to complete this dissertation. I am grateful to Dr. Zaichun Feng for his immense help and valuable guidance during my $\mathrm{PhD}$ study. I take this opportunity to thank my committee members, Dr. Satish Nair and Dr. Robert O'Connell for their time and consideration in reading my dissertation and for their continuous help in defining and completing my dissertation.

I would like to extend my thanks to my colleagues Dr. Qi Cheng, Dr. Yifan Wu, Dr. Muhammad Hai, Ghosh Shibajyoti, Nuh Yuksek, Feng Feng, Yushan Chang, Lining Huang and Chen Chen for their help and support during my research. I would also thank Jianxiong Zhu for his valuable help and support in this research project.

I specially want to thank my parents Zhiqiang Lin and Aizhu Chen, my parents in law Yongfu Xie and Yingxia Wu for their encouragement, love and support throughout my life. Finally, I would thank my wife Hui Xie for her love and support all the time during my PhD study. Big thanks to her encouragement and company which keeps me moving forward. 


\section{TABLE OF CONTENTS}

ACKNOWLEDGEMENTS .............................................................................. ii

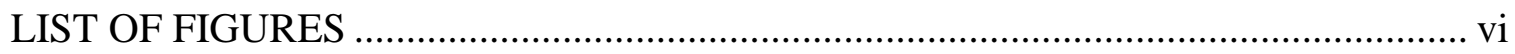

LIST OF TABLES ........................................................................................... xii

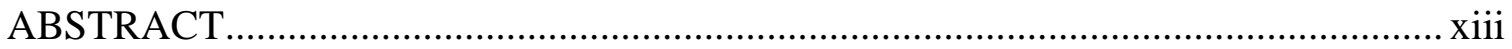

CHAPTER 1 INTRODUCTION AND MOTIVATION …....................................... 1

1.1 Microelectromechanical systems (MEMS) ................................................. 1

1.2 Review of the principles for energy harvesting ........................................... 2

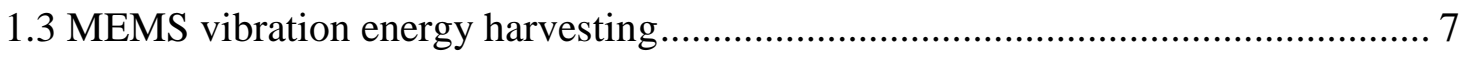

1.4 MEMS electrostatic energy harvester........................................................ 17

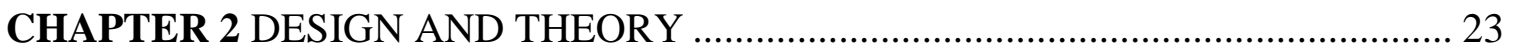

2.1 Designs of the MEMS variable capacitor with dual-cavity for power harvesting.. 23

2.1.1 Design 1: Single MEMS variable capacitor with two-cavity ........................ 23

2.1.2 Design 2: MEMS variable capacitor array with two-cavity ........................... 26

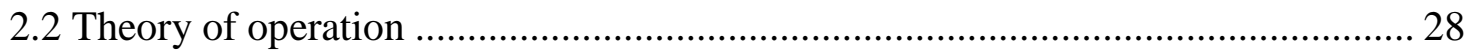

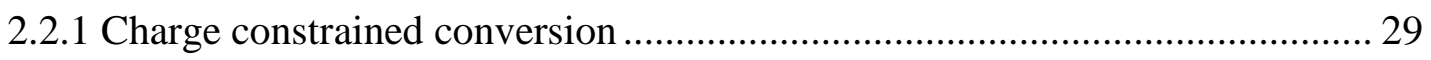

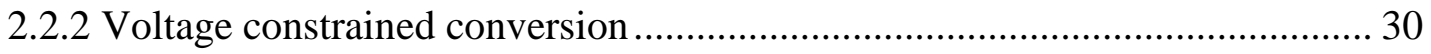

2.2.3 Modified voltage constrained strategy ................................................. 30

2.3 Theoretical background of parallel plates variable capacitor .............................. 32

2.3.1 General model of parallel plates variable capacitor................................... 33

2.3.2 Model of electrical capacitance with dielectric layer................................. 35

2.3.3 Model of electrostatic force of capacitor with dielectric layer ...................... 37

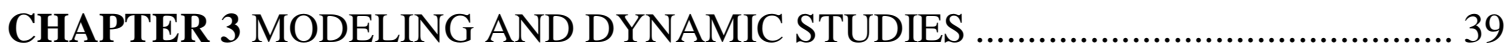




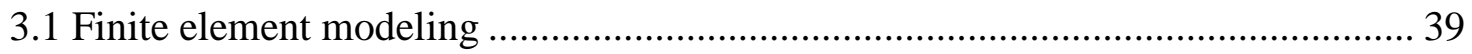

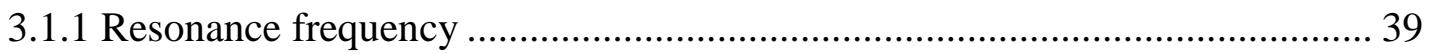

3.1.2 Nonlinear effects of squeeze film air damping, electrostatic force and elastic

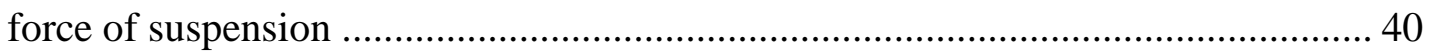

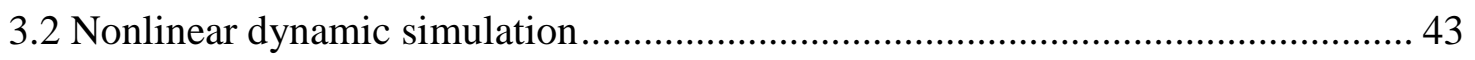

3.2.1 Dynamic simulation of MEMS variable capacitor with single cavity and two-

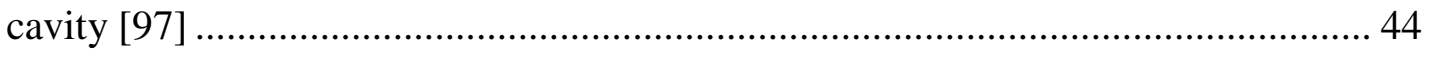

3.2.2 Dynamic simulation of MEMS variable capacitor with two-cavity involving

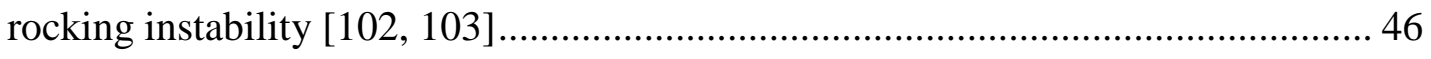

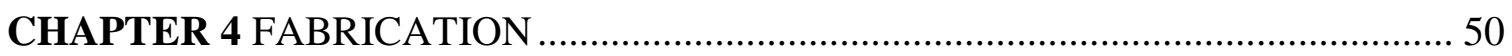

4.1 Microfabrication techniques and materials .......................................................... 50

4.2 Fabrication process of MEMS variable capacitor with two-cavity ......................... 64

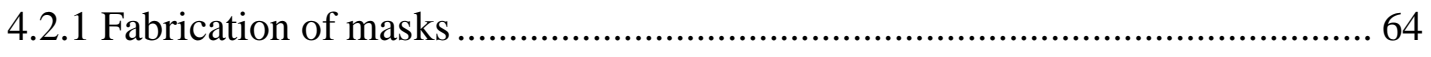

4.2.2 Fabrication of the device with a movable plate and suspension beams thickness

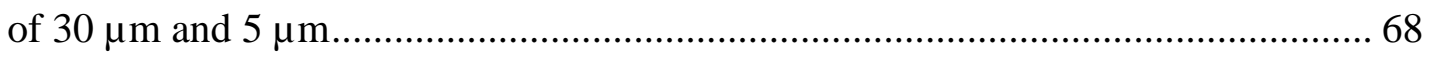

4.2.3 Fabrication of the device with a movable plate and suspension beams thickness

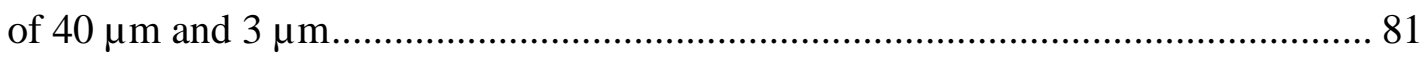

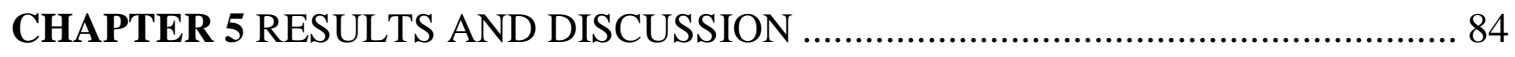

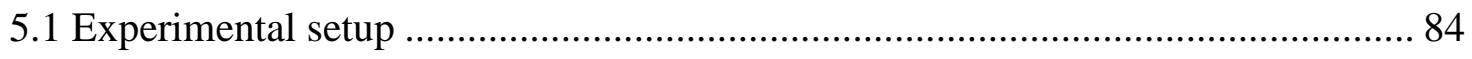

5.2 Frequency sweep testing at fixed excitation amplitude ....................................... 87

5.2.1 Power harvesting devices with a movable plate and suspension beams

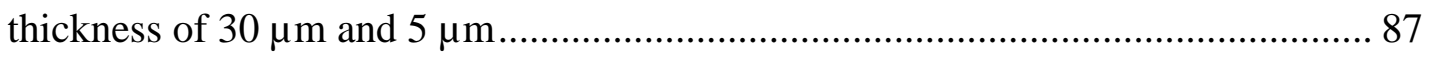


5.2.2 Power harvesting devices with a movable plate and suspension beams

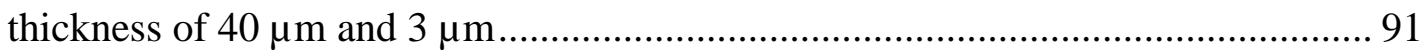

5.2.3 Comparison of the results in frequency sweep testing at fixed excitation

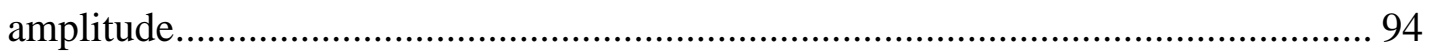

5.3 Excitation sweep testing at the resonance frequency....................................... 94

5.3.1 Power harvesting devices with a movable plate and suspension beams

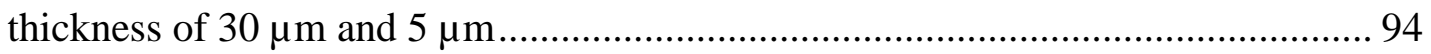

5.3.2 Power harvesting devices with a movable plate and suspension beams

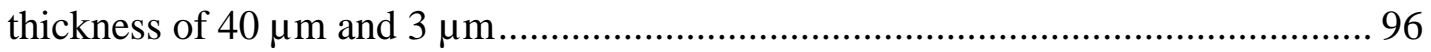

5.4 Frequency sweep testing with increasing excitation................................... 101

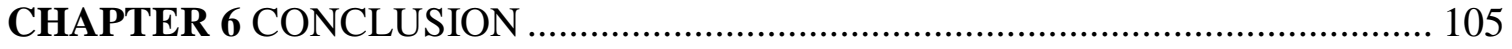

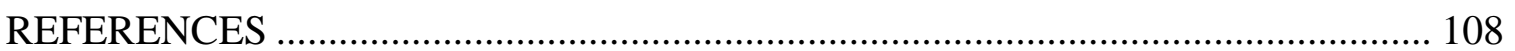

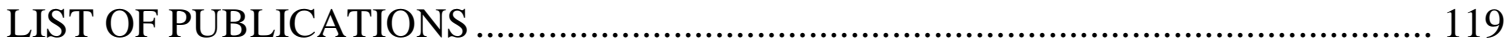

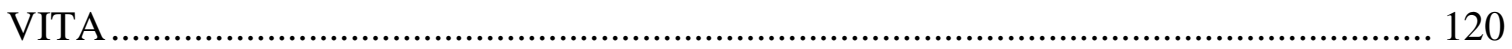




\section{LIST OF FIGURES}

Figure 1.1. A millimeter-size photovoltaic energy harvester reported by Bermejo et al. [30] [31], (a) A processed 4 inches SOI wafer showing the topologies of miniature photovoltaic harvesters with an array of 9, 25, 49 and 169 photovoltaic cells in series, (b) prototype consisting of 25 cells in series photovoltaic module and a micro-battery.......... 4

Figure 1.2. A full-fledged wearable miniaturized thermoelectric generator reported by Hoof et al. [34], (a) photo of micromachined thermopile chips placed on one Euro coin, (b) a SEM micrograph of a rim structure where thousands of thermocouples are fabricated in a daisy chain, (c) close-up view of the micromachined thermocouples........ 6

Figure 1.3. RF energy harvesting, (a) Optimized design of $2.45 \mathrm{GHz}$ microstrip rectenna boards developed by Vullers et al., back and front views [37], (b) wireless charging solution by Powercast: an RF transmitter is plugged into a wall socket, it radiates radio waves to tiny receivers integrated into devices [38]. 7

Figure 1.4. A generic model of vibration energy harvesters [40] 9

Figure 1.5. General prototypes of electromagnetic energy harvester [40]. 11

Figure 1.6. Several electromagnetic MEMS energy harvesters developed by other research groups, (a) an electromagnetic energy harvester [56], (b) a sandwiched electromagnetic energy harvester [57].

Figure 1.7. The piezoelectric effect: voltage induced by mechanical strains in piezoelectric material. (a) 33 mode, (b) 31mode [40]. 14

Figure 1.8. Various piezoelectic vibration energy harvester reported in recent years, (a) a low frequency energy harvester with piezoelectric cantilever reported by Xu et al. [66], (b) a low-frequency meandering piezoelectric vibration energy harvester presented by Berdy et al. [67]. 16

Figure 1.9. Electrostatic generators using three different topologies micromachined variable capacitors, (a) in-plane overlap, (b) in-plane gap closing, and (c) out-of-plane gap closing [71]. 18

Figure 1.10. In-plane and out-of-plane electrostatic energy harvesters, (a) an in-plane MEMS electrostatic transducer using interdigitated comb fingers structure [74], (b) nonlinear curved springs and comb fingers designed for an in-plane MEMS electrostatic energy harvester [80], (c) a low frequency out-of-plane MEMS electrostatic energy harvester using a Si proof mass suspended with polyimide [81, 82].... 19 
Figure 1.11. The most recent reported prototypes of electrostatic MEMS energy harvesters with, (a) nonlinear springs developed by Nguyen et al. [80, 89], (b) an in-plane vibration-driven using electret layer reported by Suzuki et al. [86-88], (c) an integrated electrostatic multi-transducer presented by Cuong and Halvorsen et al. [90, 91] ........... 21

Figure 2.1. 3D Schematic of single MEMS variable capacitor with dual-cavity, (a) front view of the whole device with all components labeled, (b) structure of movable plate, (a) different angle view of the whole device, top substrate is risen up and made transparent for show. 24

Figure 2.2. (a) Dimensions of the perforated movable plate and serpentine suspension beams, (b) Dimension of the mechanical stoppers on the fixed plates. 25

Figure 2.3. A 3D Schematic of MEMS variable capacitor array with dual-cavity, top substrate is risen up and made transparent for show. 27

Figure 2.4. A charge-voltage plane of the variable capacitor for energy harvesting showing the charge-constrained and voltage constrained cycles [70]. 28

Figure 2.5. A schematic of operation principle for MEMS variable capacitor with twocavity. 31

Figure 2.6. A schematic of the simplest model of parallel plate variable capacitor. 34

Figure 2.7. (a) The model capacitor incorporating the dielectric layer, (b) Capacitances as functions of the plate displacement. Purple: without dielectric layer. Blue: with dielectric layer and $\mathrm{De}=0.81$. 36

Figure 3.1. Finite Element Modeling of the suspended movable plate under fixed excitation amplitude. The plate and suspension beam thickness, and the determined resonance frequency were: (a) $30 \mu \mathrm{m}, 5 \mu \mathrm{m}$, and $487 \mathrm{~Hz}$, (b) $50 \mu \mathrm{m}, 5 \mu \mathrm{m}$, and $372 \mathrm{~Hz}$, and (c) $40 \mu \mathrm{m}, 3 \mu \mathrm{m}$, and $205 \mathrm{~Hz}$. 41

Figure 3.2. A modeling schematic of conventional single cavity energy harvester showing the arrangement of springs and electrical control circuit [97]. 44

Figure 3.3. A modeling schematic of two-cavity energy harvester showing the arrangement of springs and electrical control circuit [97]. 45

Figure 3.4. A modeling schematic of electrostatic MEMS energy harvester with dualcavity involving rocking instability [103] 47 
Figure 4.1. (a) Karl-Suss MA6-BA6 Mask Aligner in the Microsystem Research Lab of the University of Missouri, (b) a schematic of the photolithography process using positive

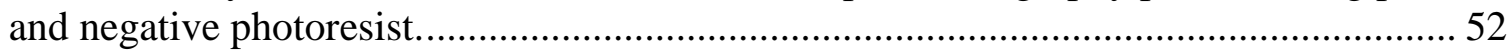

Figure 4.2. The isotropic and anisotropic etching profile 54

Figure 4.3. (a) The Oxford Plasmalab 80 Reactive Ion Etcher in the Microsystem Research Lab of University of Missouri, (b) a typical capacitively coupled plasma RIE configuration. 57

Figure 4.4. (a) A typical magnetron sputtering system configuration, (b) Kurt Lesker AXXIS Multi-Deposition System in the Microsystem Research Lab of University of Missouri. 59

Figure 4.5. A schematic of the RF magnetron sputtering magnetic poles and field. 60

Figure 4.6. A schematic and photos of the experimental setups used for $\mathrm{Ni}$ and $\mathrm{Au}$ electroplating, (a) the electroplating process, (b) $\mathrm{Ni}$ electroplating, and (c) $\mathrm{Au}$ electroplating. 62

Figure 4.7. A standard Lift-off process for pattern generation using negative photoresist. 63

Figure 4.8. Arrangement of layouts of design 1 and design 2 on a 3" silicon wafer. 65

Figure 4.9. The layouts of design 1 and design 2 on the seven masks, (a) mask of anchors and bonding tabs, (b) mask of fixed plates, (c) mask of stoppers, (d) mask of dielectrics, (e) mask of movable plate with suspension beams, (f) magnified layout of movable plate with suspension beams, (g) mask of movable plate, (h) mask of bonding tabs 67

Figure 4.10. (a) The sideview schematic shows the substrate cleaning and RIE etching for anchors and bonding tabs, (b) the sideview schematic shows the thermal oxidation to grow the insulation layer, (c) optical pictures of the square trenches for anchors after thermal oxidation, (d) a magnified image of the square trench for an anchor. 69

Figure 4.11. (a) The sideview schematic shows the $\mathrm{Cr}$ and $\mathrm{Au}$ thin films are sputter deposited, (b) the sideview schematic shows the Au thin film patterning for bottom plates, trace lines and seed layer for anchors and bonding tabs, (c) \& (d) optical pictures of the square trenches for anchors after $\mathrm{Cr}$ and $\mathrm{Au}$ sputter depositions, (e) \& (f) optical pictures of patterned fixed plates, anchors and trace lines. 71

Figure 4.12. (a) The sideview schematic shows the electroplated Au stoppers, (b) the sideview schematic shows the sputtered and patterned $\mathrm{SiO}_{2}$ and $\mathrm{Si}_{3} \mathrm{~N}_{4}$ dielectric layers, (c) 
\& (d) optical pictures of the electroplated Au stoppers on the plates, (e) \& (f) optical pictures of the plates with $\mathrm{Au}$ stoppers after being covered by $\mathrm{SiO}_{2}$ and $\mathrm{Si}_{3} \mathrm{~N}_{4}$ dielectric layers. 73

Figure 4.13. (a) The sideview schematic shows the Ni electroplating for anchors and bonding tabs, (c) optical pictures of the electroplated Ni anchors, (d) a magnified image of an electroplated $\mathrm{Ni}$ anchor. 74

Figure 4.14. (a) The sideview schematic shows the sputter depositions of $\mathrm{Cr}$ and $\mathrm{Au}$ thin films for the second seed layer and forming movable plate with serpentine suspension beam by Ni electroplating, (b) \& (c) optical pictures of the Ni electroplated movable plate with serpentine suspension beams. 75

Figure 4.15. (a) The sideview schematic shows the Ni electroplating to increase the thickness of the movable plate, (b) \& (c) optical pictures of the movable plate with increased thickness, and without changing the thickness of suspension beams. 76

Figure 4.16. (a) The sideview schematic shows the releasing all photoresist layers, (b) \& (c) optical pictures of the movable plate with suspension beams after photoresist releasing.

Figure 4.17. (a) The sideview schematic shows the fabricating top substrate with In bonding layer, (b) the sideview schematic shows the forming the final device by In bonding. 78

Figure 4.18. SEM micrographs of the MEMS variable capacitors with a $30 \mu \mathrm{m}$ thick movable plate and $5 \mu \mathrm{m}$ thick suspension beams before and after bonding the top cavity, (a) a $30 \mu \mathrm{m}$ thick movable plate, (b) the magnified view of a $5 \mu \mathrm{m}$ thick serpentine suspension beam, (c) the magnified view of the movable plate's edge and the $30 \mu \mathrm{m}$ air gap, (d) the $30 \mu \mathrm{m}$ height capacitor anchors and the $4 \mu \mathrm{m}$ height stoppers on the fixed plate, (e) the $1 \times 4$ capacitor array before bonding the top cavity, (f) the bonded device with two-cavity. 80

Figure 4.19. SEM micrographs of the MEMS variable capacitors with a $40 \mu \mathrm{m}$ thick movable plate and $3 \mu \mathrm{m}$ thick suspension beams before and after bonding the top cavity. (a) a $40 \mu \mathrm{m}$ thick movable plate, (b) the magnified view of $3 \mu \mathrm{m}$ thick serpentine suspension beam and $30 \mu \mathrm{m}$ air gap, (c) a magnified view of $3 \mu \mathrm{m}$ thick serpentine suspension beam captured from a different angle, (d) the movable plate's edge and $30 \mu \mathrm{m}$ air gap. 82

Figure 5.1. The schematic of the electrical characterization circuit. 85

Figure 5.2. The experimental testing setup, (a) an overview of testing setup and environment, (b) the PCB test stage mounted on the shaker. 86 
Figure 5.3. Time domain plots of AC output voltage across the load resistors from the devices with a movable plate and suspension beams thickness of $30 \mu \mathrm{m}$ and $5 \mu \mathrm{m}$, respectively, (a) single capacitor device under $15 \mathrm{~V} \mathrm{DC}$ bias, $300 \mathrm{~Hz}$ and $1.026 \mathrm{~g}$ excitation, (b) $1 \times 4$ capacitor array device under $15 \mathrm{~V} \mathrm{DC}$ bias, $300 \mathrm{~Hz}$ and $1.020 \mathrm{~g}$ excitation. 88

Figure 5.4. The RMS output voltage from bottom cavity of the devices with a movable plate and suspension beams thickness of $30 \mu \mathrm{m}$ and $5 \mu \mathrm{m}$, respectively, as frequency sweeps, (a) a single capacitor under RMS excitation of $1.026 \mathrm{~g}$, (b) $1 \times 4$ capacitor array under RMS excitation of $1.020 \mathrm{~g}$. 89

Figure 5.5. The total power across the load resistors from the devices with a movable plate and suspension beams thickness of $30 \mu \mathrm{m}$ and $5 \mu \mathrm{m}$, respectively, as frequency sweeps, (a) a single capacitor under RMS excitation of $1.026 \mathrm{~g}$, (b) $1 \times 4$ capacitor array under RMS excitation of $1.020 \mathrm{~g}$. 90

Figure 5.6. The RMS output voltage from bottom cavity of the devices with a movable plate and suspension beams thickness of $40 \mu \mathrm{m}$ and $3 \mu \mathrm{m}$, respectively, as frequency sweeps, (a) a single capacitor under RMS excitation of $1.041 \mathrm{~g}$, (b) $1 \times 4$ capacitor array under RMS excitation of $1.018 \mathrm{~g}$. 92

Figure 5.7. The total power across two load resistors from the devices with a movable plate and suspension beams thickness of $40 \mu \mathrm{m}$ and $3 \mu \mathrm{m}$, respectively, as frequency sweeps, (a) a single capacitor under RMS excitation of $1.041 \mathrm{~g}$, (b) $1 \times 4$ capacitor array under RMS excitation of $1.018 \mathrm{~g}$. 93

Figure 5.8. Comparisons of the results in frequency sweep testing with a DC bias of $15 \mathrm{~V}$, (a) the RMS output voltage from bottom cavity of the devices as frequency sweeps under RMS excitation around $1 \mathrm{~g}$, (b) the total power across two load resistors as frequency sweeps under RMS excitation around $1 \mathrm{~g}$.. 95

Figure 5.9. The RMS output voltage from bottom cavity of the devices with $30 \mu \mathrm{m}$ movable plate and $5 \mu \mathrm{m}$ suspension beams as excitation sweeps under a fixed frequency of $500 \mathrm{~Hz}$, (a) the RMS output voltage from single capacitor device as function of excitation acceleration, (b) the RMS output voltage from $1 \times 4$ capacitor array device as function of excitation acceleration. 97

Figure 5.10. The total power across two load resistors from the devices with $30 \mu \mathrm{m}$ movable plate and $5 \mu \mathrm{m}$ suspension beams as excitation sweeps under a fixed frequency of $500 \mathrm{~Hz}$, (a) total power generated by single capacitor device as function of excitation acceleration, (b) total power generated by $1 \times 4$ capacitor array device as function of excitation acceleration. 98 
Figure 5.11. The RMS output voltage from bottom cavity of the devices with $40 \mu \mathrm{m}$ movable plate and $3 \mu \mathrm{m}$ suspension beams as excitation sweeps under a fixed frequency of $500 \mathrm{~Hz}$, (a) the RMS output voltage from single capacitor device as function of excitation acceleration, (b) the RMS output voltage from $1 \times 4$ capacitor array device as function of excitation acceleration.

Figure 5.12. The total power across two load resistors from the devices with $40 \mu \mathrm{m}$ movable plate and $3 \mu \mathrm{m}$ suspension beams as excitation sweeps under a fixed frequency of $290 \mathrm{~Hz}$, (a) total power generated by single capacitor device as function of excitation acceleration, (b) total power generated by $1 \times 4$ capacitor array device as function of excitation acceleration. 100

Figure 5.13. The RMS output voltage from bottom cavity of the devices as frequency sweeps under increasing excitation acceleration, (a) the RMS output voltage from single capacitor, (b) the RMS output voltage from $1 \times 4$ capacitor array. 102 


\section{LIST OF TABLES}

Table 1. Characteristics of various energy sources available in the ambient [26]............ 3

Table 2. The device dimensions used in the fabrication for design 1 ............................ 26

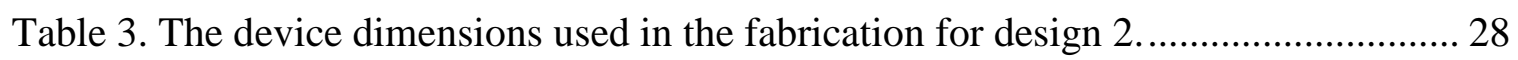

Table 4. The expected resonant frequencies of the power harvesting devices with various dimensions determined using FEM Modeling...................................................... 42

Table 5 Specifications that are used in the Matlab simulation for single cavity and two cavity models. The two cavity model has a $30 \mu \mathrm{m}$ top air gap. The electroplated Nickel density is $8912 \mathrm{~kg} / \mathrm{m}^{3}$

Table 6. Comparison of simulation results of average power (Average Current $\times$ Output Voltage) produced by a single cavity model and a two-cavity model [97]. ................... 46

Table 7. Comparison of simulation results of average power (Average Current $\times$ Output Voltage) produced by a two-cavity model without and with rocking instability [103]... 48

Table 8. The seven masks set used in the fabrication process of MEMS variable capacitor with two-cavity. 68

Table 9. Summary of the dimensions of all fabricated devices. ................................... 83

Table 10. Summary of the testing results of all fabricated devices. 101 


\begin{abstract}
In this research, a novel MEMS variable capacitor with two capacitive cavities for energy harvesting was developed that use the wasted energy associated with undesirable mechanical vibrations to power microelectronic sensors and actuators widely found in structures and systems surrounding us. The harvested power, though very small, can have a profound effect on the usage of microsensors. First, the self-powered sensors will no longer require regular battery maintenance. Second, the self-powered chip is a liberating technology. On a circuit board, it can simplify the connection. On a commercial jet, the sensors can greatly simplify cabling.

The design, fabrication, modeling and complete set of characterization of MEMS variable capacitors with two-cavity are presented in details in this thesis. The MEMS variable capacitors are unique in its two-cavity design and use of electroplated nickel as the main structural material. The device consists of $2 \times 2 \mathrm{~mm}^{2}$ movable capacitive proof mass plates with a thickness of $30 \mu \mathrm{m}$ suspended between two fixed electrodes forming two vertical capacitors. When the capacitance increases for one cavity, it decreases for the other. This allows using both up and down directions to generate energy. The suspended movable plates are supported by four serpentine springs with a thickness of 3$5 \mu \mathrm{m}$ that are attached to the address lines on a silicon substrate only at the anchors' points which is made of electroplated nickel. The serpentine suspension beams are made with a width, thickness and total length (four serpentine turns) of $15 \mu \mathrm{m}, 5 \mu \mathrm{m}$ and 1485 $\mu \mathrm{m}$. Five gold stoppers with height of $2-4 \mu \mathrm{m}$ were electroplated on the fixed plates to prevent snap-down of the movable plates by overwhelming electrostatic force. $\mathrm{SiO}_{2}$ and
\end{abstract}


$\mathrm{Si}_{3} \mathrm{~N}_{4}$ thin layers were patterned on the fixed plates to insulate the stoppers and enhance the dielectric property of capacitive cavities.

The MEMS variable capacitor with two-cavity has been designed and modeled using MEMS CAD tool and COMSOL Multi-Physics finite element tool. The designed structure was confirmed with good mechanical durability and appropriate resonance response. Numerical programs that are able to simulate the dynamic motion of the movable plate and calculate the total harvested power for given vibration amplitude and frequencies were developed using Matlab by Dr. Zaichun Feng's research team. The twocavity MEMS capacitors have achieved higher average power than that of single-cavity devices under a wide range of vibration frequencies and amplitudes based on the timedomain simulations [93]. Rocking instability, i.e. perturbations that may result in movable plates not in parallel to fixed plates, of the movable plate also has been studied by their research base upon comprehensive dynamic simulations in Matlab. Resultant symmetric breaking can cause drastic reduction on power generation [98] [99].

A single MEMS variable capacitor and $1 \times 4$ capacitor array with two capacitive cavities for energy harvesting from environmental vibration has been successfully fabricated using surface micromachining technology and were fully characterized using a printed circuit board test stage mounted on a shaker. The comparison of the output performance between conventional single cavity device and new two-cavity devices has been made. The results indicate that the two-cavity device has achieved considerably higher energy generation than conventional single cavity device under various vibrations. Experimentations were also implemented to exploit an enhanced performance of power generation using $1 \times 4$ capacitor array. It is observed that the measured RMS voltage 
from capacitor array is 3.2 times in average of that from single capacitor under extensive excitation conditions, which indicates that the capability of power generation from capacitor array is 3.2 times higher in average than that of a single capacitor due to its higher variation of capacitance. The measured power across the load resistors from the single capacitor and from the capacitor array achieved a maximum power around $220 \mathrm{nW}$ and $2.5 \mu \mathrm{W}$ respectively under a high DC bias of $15 \mathrm{~V}$ and extremely intense excitation above $5 \mathrm{~g}$. The measured resonance frequency of the devices was around $500 \mathrm{~Hz}$. However, it is noted that the resonance frequency of the capacitor array is reported with broader bandwidth response than that of a single capacitor due to slightly different resonance response from each capacitor. The resonance frequency of the devices was decreased from approximately $500 \mathrm{~Hz}$ to $470 \mathrm{~Hz}$ as the excitation acceleration was increased from $0.5 \mathrm{~g}$ to $2.5 \mathrm{~g}$. This might be due to nonlinear effects of squeezed film air damping, electrostatic force and elastic force of suspension.

In addition, a single MEMS variable capacitor and $1 \times 4$ capacitor array with heavier movable proof mass plate and less stiff serpentine suspension beams were also developed for lower frequency application. In this case, the movable plate with serpentine suspension beams is created by electroplating $\mathrm{Ni}$ for $3 \mu \mathrm{m}$ (instead of $5 \mu \mathrm{m}$ ) inside of a $10 \mu \mathrm{m}$ AZ-P4620 photoresist mold, then another AZ-P4620 photoresist layer with a thickness of $30 \mu \mathrm{m}$ is added on the previous $10 \mu \mathrm{m}$ AZ-P4620 photoresist mold in order to increase the total thickness of the movable plate up to $40 \mu \mathrm{m}$. Therefore, the movable proof mass plate thickness was increased to $40 \mu \mathrm{m}$ while the thickness of suspension beams was reduced to $3 \mu \mathrm{m}$. The testing results demonstrate a lower resonance frequency around $290 \mathrm{~Hz}$, and the measured total power across the load resistors from the device of 
single capacitor and the device of capacitor array achieved a maximum power of $216 \mathrm{nW}$ and $2.14 \mu \mathrm{W}$, respectively, under a high DC bias of $15 \mathrm{~V}$ and extremely intense excitation above $3.7 \mathrm{~g}$. 


\section{CHAPTER 1}

\section{INTRODUCTION AND MOTIVATION}

This chapter presents a brief history and evolution of Microelectromechanical systems (MEMS) technology. The recent development of MEMS energy harvesters using different mechanisms will be introduced. The advantages and disadvantages of each mechanism will be comprehensively discussed based upon existing prototypes of MEMS energy harvesters in published literatures.

\subsection{Microelectromechanical systems (MEMS)}

Microelectromechanical systems (MEMS) technology is referred to a process technology used to develop micro devices or systems which combine mechanical and electrical components together with electronic circuits, typically on a semiconductor chip. MEMS devices are fabricated using integrated circuit (IC) batch fabrication techniques and can range in size from sub-micron level to millimeter level, and they are highly integratable for any number, from a few to millions, in particular systems or applications. MEMS devices have the capability of sensing, controlling and actuating in the micro world, and then producing responses or effects in the macro scale. MEMS has been recognized as one of the most promising technologies in the $21^{\text {st }}$ century, and many existing commercial MEMS devices have revolutionized both industrial and consumer products by combining silicon-based microelectronics with micromachining technology. MEMS technology and MEMS based devices have dramatically affected all of our lives and the way we live [1-2]. 
Nowadays, MEMS devices are fabricated using much more advanced semiconductor and IC fabrication technologies such as wet etching [3], photolithography [4] [5], reactive ion etching/deep reactive ion etching [6-8], plasma enhanced chemical vapor deposition [9, 10], ion implantation [11], etc. A variety of MEMS devices have already been developed and commercialized in the last 20 years such as microreservoirs [12], micropump [13, 14], micromixer [15], cantilever [16-18], rotors [19], microchannels [20, 21], microvalve [22] and microsensors [23-25].

\subsection{Review of the principles for energy harvesting}

Rechargeable batteries are used for powering most portable and wireless sensors and electronics, with their size determining their lifespan and autonomy. They have limited energy capacity, are expensive, heavy, and require periodic recharging and maintenance or replacement. These batteries seriously limit the total system with low power to operate. Thus, there exists a significant drive to miniaturize the size and reduce the weight of power generators. Scavenging energy from the ambient sources, for examples, ambient light, vibration/motion energy, thermal energy, RF radiation energy, using miniature technology—specifically the technology based on MEMS—has received considerable attention worldwide. These energy harvesting devices will reduce the hazardous waste associated with batteries, and can be designed not only for power harvesting but for sensing functionalities not possible with batteries.

In the last decades, a large number of research groups have investigated and developed devices for power harvesting from environmental sources. Table 1 summarizes the output power that could be obtained from environmental sources when using micro devices built with the currently available transducer mechanisms [26]. Energy can be 
Table 1. Characteristics of various energy sources available in the ambient [26].

\begin{tabular}{|l|l|l|l|}
\hline \multicolumn{2}{|l|}{ Energy Source } & Source Power $\left(\mathbf{m W} / \mathbf{c m}^{2}\right)$ & $\begin{array}{l}\text { Harvested Power } \\
\left(\boldsymbol{\mu W} / \mathbf{c m}^{2}\right)\end{array}$ \\
\hline \multirow{2}{*}{ Ambient light } & Indoor & 0.1 & 10 \\
\cline { 2 - 4 } & Outdoor & 100 & $10 \times 10^{3}$ \\
\hline $\begin{array}{l}\text { Vibration/motion } \\
\text { Energy }\end{array}$ & Human & $0.5 @ 1 \mathrm{~Hz} 1 \mathrm{~m} / \mathrm{s}^{2} @ 50 \mathrm{~Hz}$ & 4 \\
\cline { 2 - 4 } & Industrial & $1 @ 5 \mathrm{~Hz} 10 \mathrm{~m} / \mathrm{s}^{2} @ 1 \mathrm{KHz}$ & 100 \\
\hline \multirow{2}{*}{ Thermal energy } & Human & 20 & 30 \\
\cline { 2 - 4 } & Industrial & 100 & $1-10 \times 10^{3}$ \\
\hline \multirow{2}{*}{ RF energy } & Cell phone & $0.3 \times 10^{-3}$ & 0.1 \\
\hline
\end{tabular}

harvested effectively between $0.1 \mu \mathrm{W}-1 \mathrm{~mW}$ as shown in Table 1 , which is applicable for powering wireless sensors [27-29]. Solar energy was utilized first for power harvesting because of the availability of many applications where miniature photovoltaic cells (PV) can be a good choice as power supply for low power consumption electronics. The first practical P-N junction photovoltaic cell was developed at Bell Laboratories in 1954. The photovoltaic cell converts incoming photons into electricity as follows: photons in ambient light hit the photovoltaic cell which is made of semiconductor materials (typically silicon), and photons are absorbed. Electrons in the semiconductor are knocked off from their atoms by photon thus causing an electric potential difference. An integration of array of photovoltaic cells will produce the desired amount of electrical energy.

As an example, a miniature photovoltaic harvester (Figure 1.1) that comprised a mini-PV module and a microbattery for low power applications was reported by Bermejo et al. [30] [31]. The fabrication process of this device is based on p-type silicon-on- 
insulator (SOI) c-Si p-type $\langle 100\rangle$ wafers. The paper reported that increasing the number of array cells from 9 to 169 makes the open circuit voltage to rise from 3.6 to $101.5 \mathrm{~V}$ with generated power densities ranging from 2.07 to $6.7 \mathrm{~mW} / \mathrm{cm}^{2}$ under $100 \mathrm{~mW} / \mathrm{cm}^{2}$ standard illumination. Therefore, photovoltaic cells are an obvious energy source for selfpowered systems in outdoor environment. Their efficiencies range from $5 \%$ to $30 \%$, depending on the material used [32]. However, the illumination levels are much lower $\left(10-100 \mu \mathrm{W} / \mathrm{cm}^{2}\right)$ for indoor use. Extensive researches has shown that, the efficiency of photovoltaic cells will drop considerably at low illumination levels and generate a surface power density actually similar to that of the thermal energy harvester and vibrational energy harvester [26].

(a)

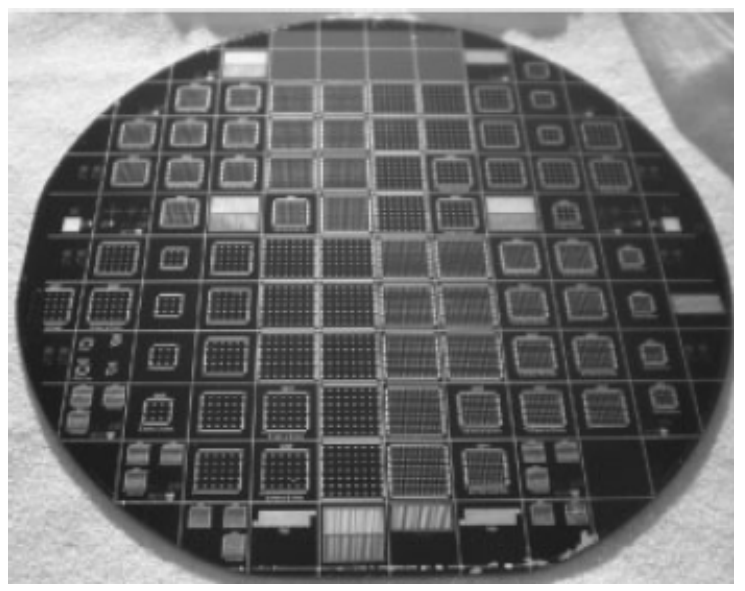

(b)

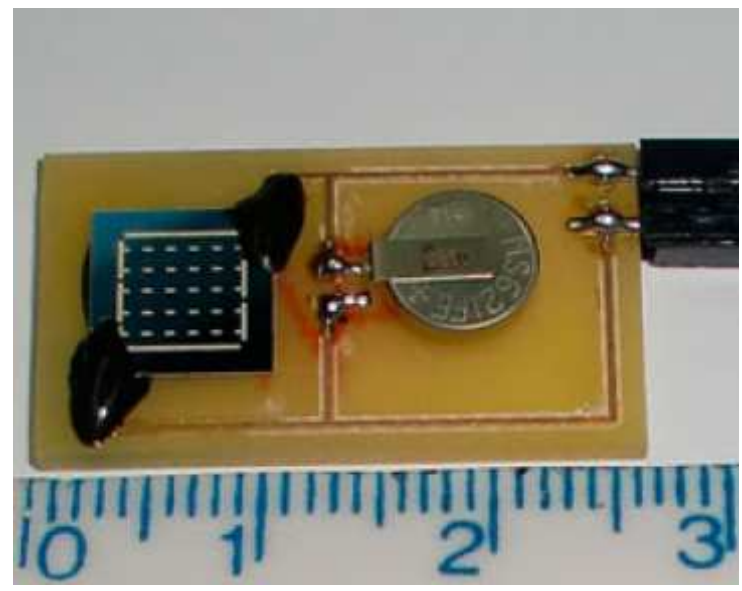

Figure 1.1. A millimeter-size photovoltaic energy harvester reported by Bermejo et al. [30] [31], (a) A processed 4 inches SOI wafer showing the topologies of miniature photovoltaic harvesters with an array of 9, 25, 49 and 169 photovoltaic cells in series, (b) prototype consisting of 25 cells in series photovoltaic module and a micro-battery.

The application of thermoelectric energy harvester for powering portable electronic devices has been utilized in the watch industry as early as in 1980's. Seiko 
presented a thermoelectric generator with thermopiles fabricated by Electric Discharge Machining (EDM) of sintered Bismuth Telluride (Bi-Te), which reported an output voltage of about $300 \mathrm{mV}$ when the watch was worn by human body. This voltage was upconverted to $1.5 \mathrm{~V}$ in order to power the electronics [33]. The Seebeck effect is responsible for the behavior of thermoelectric power harvester. It is defined as a voltage difference between two thermocouple junctions made of two dissimilar conductors or semiconductors when they are kept in a temperature differences. The core component of a thermoelectric power harvester is the thermopile which is formed by thousands of thermocouples placed between a hot and a cold plate and connected thermally in parallel and electrically in series. When there is a temperature difference $\Delta \mathrm{T}$ between the thermocouple junctions, an output voltage $\mathrm{V}$ is delivered between the two terminals due to the Seebeck effect: $\mathrm{V}=\alpha_{1} \Delta \mathrm{T}+\alpha_{2} \Delta \mathrm{T}, \alpha_{1}$ and $\alpha_{2}$ are material dependent quantities, known as Seebeck coefficients.

Hoof and his group [34] realized a full-fledged wearable miniaturized thermoelectric generator (TEG) specifically engineered for human body applications (see Figure 1.2). It is based on a surface micromachined poly-SiGe thermopile. Being worn on human body, the wearable thermoelectric power harvester delivers an open-circuit output voltage of about $0.15 \mathrm{~V}$ and an output power of about $0.3 \mathrm{nW}$ on a matched external load [34]. Although the micro thermoelectric power harvester is environmentally friendly in recycling wasted heat energy and integratable with small-sized wireless sensors, it always requires a relatively constant heat source which limits its application in variety of the environments.

Ambient RF radiation energy is also a possible source for energy harvesting. The 
(a)

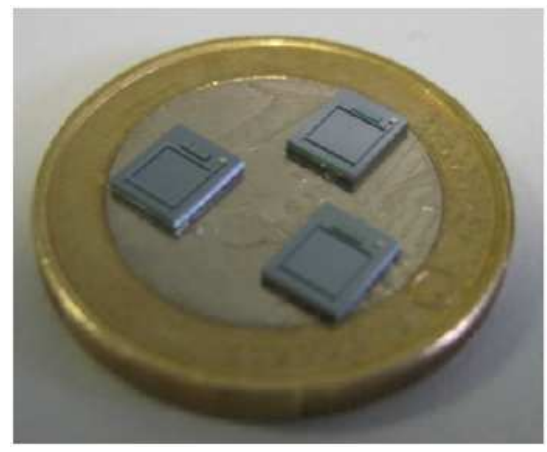

(b)

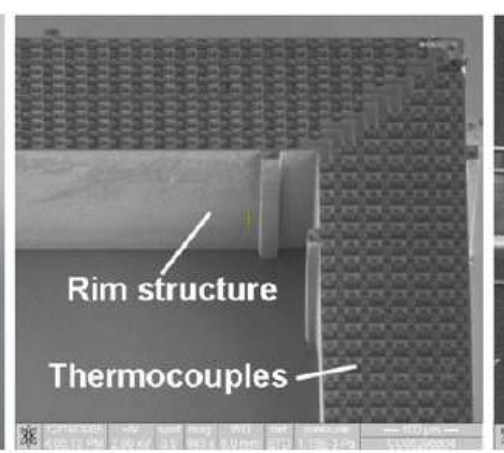

(c)

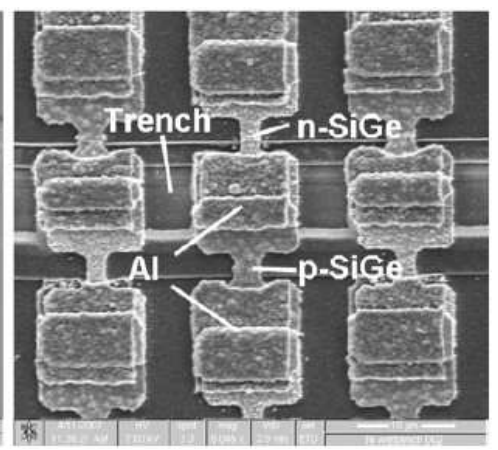

Figure 1.2. A full-fledged wearable miniaturized thermoelectric generator reported by Hoof et al. [34], (a) photo of micromachined thermopile chips placed on one Euro coin, (b) a SEM micrograph of a rim structure where thousands of thermocouples are fabricated in a daisy chain, (c) close-up view of the micromachined thermocouples.

RF energy harvesting makes use of electromagnetic radiation available through public telecommunication service such as GSM, WLAN, Wifi, etc. to generate electrical energy. Although the public RF radiation in GSM or WLAN band is extensively available in ambient, it has low power density levels which normally falls within micro watt scale. For examples, the power density levels ranging from 0.1 to $1.0 \mathrm{~mW} / \mathrm{m}^{2}$ may be expected for single frequencies for a distances ranging from $25 \mathrm{~m}$ to $100 \mathrm{~m}$ from a GSM base station [35], and the measurements in a WLAN environment indicate power density levels that are even one order of magnitude lower [36]. Therefore, neither GSM nor WLAN are likely to produce enough ambient RF energy for wirelessly powering miniature sensors unless a large area is used [26]. Alternatively, it is feasible to broadcast an electromagnetic radiation signal at a specific wavelength to charge the internal battery of electronic devices or directly power the wireless sensor nods. For examples, Vullers et al. developed a device for RF harvesting using optimized antenna structures (Figure 
1.3(a)). A received power of $1.5 \mathrm{~mW}$ at $20 \mathrm{~cm}$ with a transmission power of $100 \mathrm{~mW}$ has been reported [37]. Also, Powercast Inc. has developed commercial wireless charger to charge mobile devices using received RF energy (Figure 1.3(b)). This product reports a receiving of $100 \mathrm{~mW}$ power using $3 \mathrm{~W}$ of transmitted power at an operating frequency of $906 \mathrm{MHz}$ and a distance between device and transmitter around $30 \mathrm{~cm}$. However, one critical shortage is the received power decreases very rapidly with distance [38]. New techniques to develop wireless chargers with high conversion efficiency for a distance range of a few meters is still in research phase.

(a)

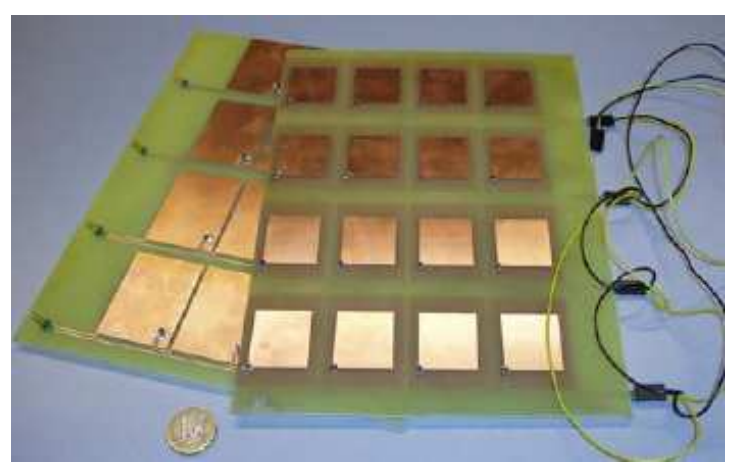

(b)

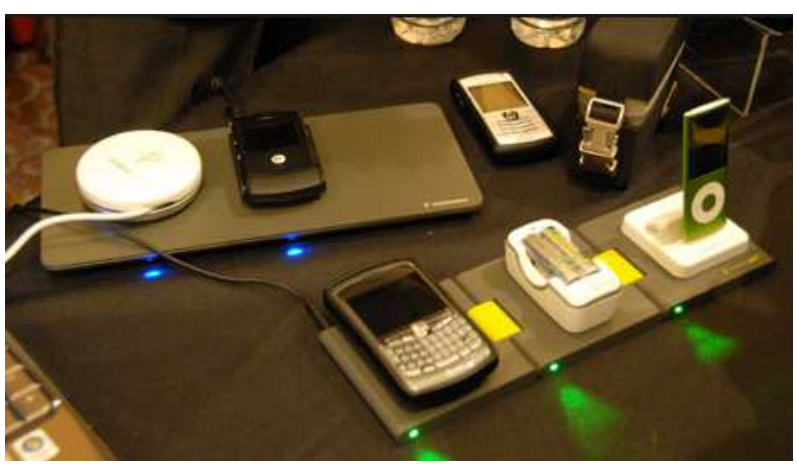

Figure 1.3. RF energy harvesting, (a) Optimized design of $2.45 \mathrm{GHz}$ microstrip rectenna boards developed by Vullers et al., back and front views [37], (b) wireless charging solution by Powercast: an RF transmitter is plugged into a wall socket, it radiates radio waves to tiny receivers integrated into devices [38].

\subsection{MEMS vibration energy harvesting}

Environmental vibration is one of the most abundant energy sources and ubiquitously available surrounding us. The environmental vibration distributes in a wide frequency range. For examples, the skyscrapers are subjected to vertical vibration in the range of $10-30 \mathrm{~Hz}$ and horizontal vibration in the range of $5-10 \mathrm{~Hz}$ induced by traffics, 
rail-networks and winds. The human motion can provide vibration during walking or running in the range of $0.5-3 \mathrm{~Hz}$. The bridges have vibration induced by traffics and winds below $40 \mathrm{~Hz}$. The engine and tire rotation of an automobile can produce vibration in the range of $5-100 \mathrm{~Hz}$ and $0-20 \mathrm{~Hz}$, respectively. The cabin of an in-flight aircraft has vibration in the range of $50-500 \mathrm{~Hz}$ and its wings are subjected to vibration below $10 \mathrm{~Hz}$. Moreover, various industry machineries can produce vibration in an extensive frequency range up to several thousands of hertz.

The easy availability of environmental vibration makes converting ambient vibration energy into electrical energy an attractive approach for powering wireless sensors. A vibration energy harvester is typically composed of a frame anchored to the vibration source and a mass connected to the frame by a suspension having a stiffness $\mathrm{k}$. The vibration induces a relative motion of the mass and the frame, a transducer transform the energy of this relative motion into electricity. Vibration based energy harvesters can be modelled as a second-order, spring-mass system. The Williams and Yates first developed a generic model of vibration energy harvesters as shown in Figure 1.4 [39] [40]. The model consists of a proof mass (m) and a spring with the spring constant (k). The proof mass moves out of phase with the harvester housing (frame) when the harvester vibrates. There is a relative displacement between the proof mass and the housing. This displacement is sinusoidal in amplitude and can drive a suitable vibration energy harvester to generate electrical energy. In Figure 1.4, $b$ is the damping coefficient that normally consists of mechanically induced damping (parasitic damping) coefficient $b_{m}$ and electrically induced damping coefficient $b_{e} . y(t)$ is the displacement of the harvester housing and $z(t)$ is the relative motion of the mass with respect to the housing. 


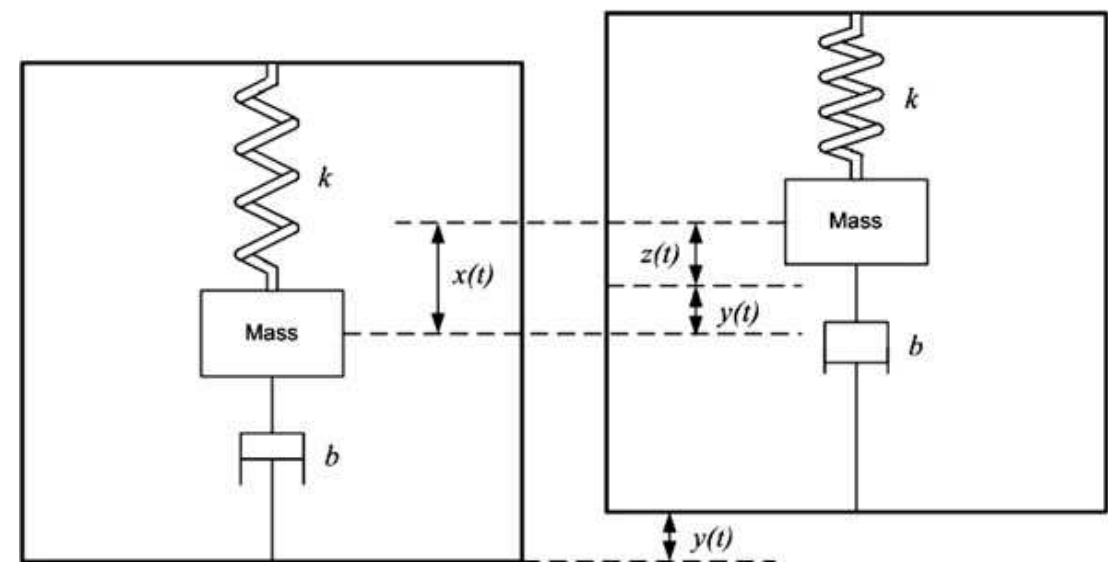

Figure 1.4. A generic model of vibration energy harvesters [40].

For a sinusoidal excitation, $y(t)$ can be written as $y(t)=Y \sin (\omega t)$, where $Y$ is the amplitude of vibration and $\omega$ is the angular frequency of vibration. The system can be described by following differential equation [40]:

$$
m \ddot{z}+\left(b+b_{p}\right) \dot{z}+k z=m \ddot{y}
$$

Where $b_{p}$ is the damping coefficient due to parasitic effects such as squeeze film air, friction of the sliding part, etc. The main characteristic of a vibration energy harvester is that it operates in resonance. The resonance frequency is given by:

$$
f=\frac{1}{2 \pi} \sqrt{\frac{k}{m}}
$$

The power in resonance for the mass spring system is given by [40]:

$$
p(f)=4 \pi^{3} m f^{3} Y z(t)_{\max }
$$

Where $z(t)_{\max }$ corresponding to the maximum displacement of the proof mass at resonance. The equation (3) indicates that large output powers can be enabled by higher external vibration frequencies and amplitude [40].

Resonant vibration energy harvesters are by far the most widely investigated in 
the literature. Micro energy harvesters for vibration-to-electric power conversion fall within three mechanism categories: electromagnetic, piezoelectric and electrostatic. Classification of the three transduction mechanisms and the principles of operation will be described in details in the section 1.3 and 1.4. Performance of some of the reported vibration energy harvesters will be summarized. Advantages and disadvantages of each mechanism will be listed and compared.

Since environmental vibration is among the most abundant energy sources, ubiquitous on automobiles, airplanes, bridges, etc, micro-energy harvesting from environmental vibration has been widely studied for fully autonomous wireless sensor networks due to its broad range of applications, including structural health monitoring, smart building, predictive maintenance, biomedical implants, structure-embedded instrumentations and automotive system [41-51]. For example, the power harvester can be attached inside a vehicle's tire in order to harvest power, typically in the $\mu$ watt range, to power the sensors that measure the tire pressure, tire damage, tire temperature, and acceleration in all directions. These data can be used to improve the performance of the antilock braking system and electronic braking distribution control system safety, and estimate real-time forces and moments during operation that can be used to evaluate the status of the road and improve the vehicle safety [52-55].

The vibration energy harvester requires a mechanical system to couple environmental displacements to the transduction mechanism. This mechanical system has to be designed to maximize the coupling between the vibration energy source and the transduction mechanism. The vibration based energy harvesters can be modeled as a second-order spring-mass system (see Figure 1.4) consisting of an inertial frame that 
transmits the vibration to a suspended inertial mass to produce a relative displacement or cause mechanical strain. The transduction mechanism can then generate electrical energy by exploiting the relative displacement or strain [40]. The MEMS vibration energy harvester use one of three transduction mechanisms: electromagnetic, piezoelectric and electrostatic.

Faraday discovered electromagnetic induction in 1831 states that an electrical current will be induced in a closed circuit when the magnetic flux through a surface bounded by the magnetic field changes in strength or the conductor is moved through the field. The MEMS electromagnetic energy harvester was commonly demonstrated as employing conventional permanent magnet moving through coils or coils integrated
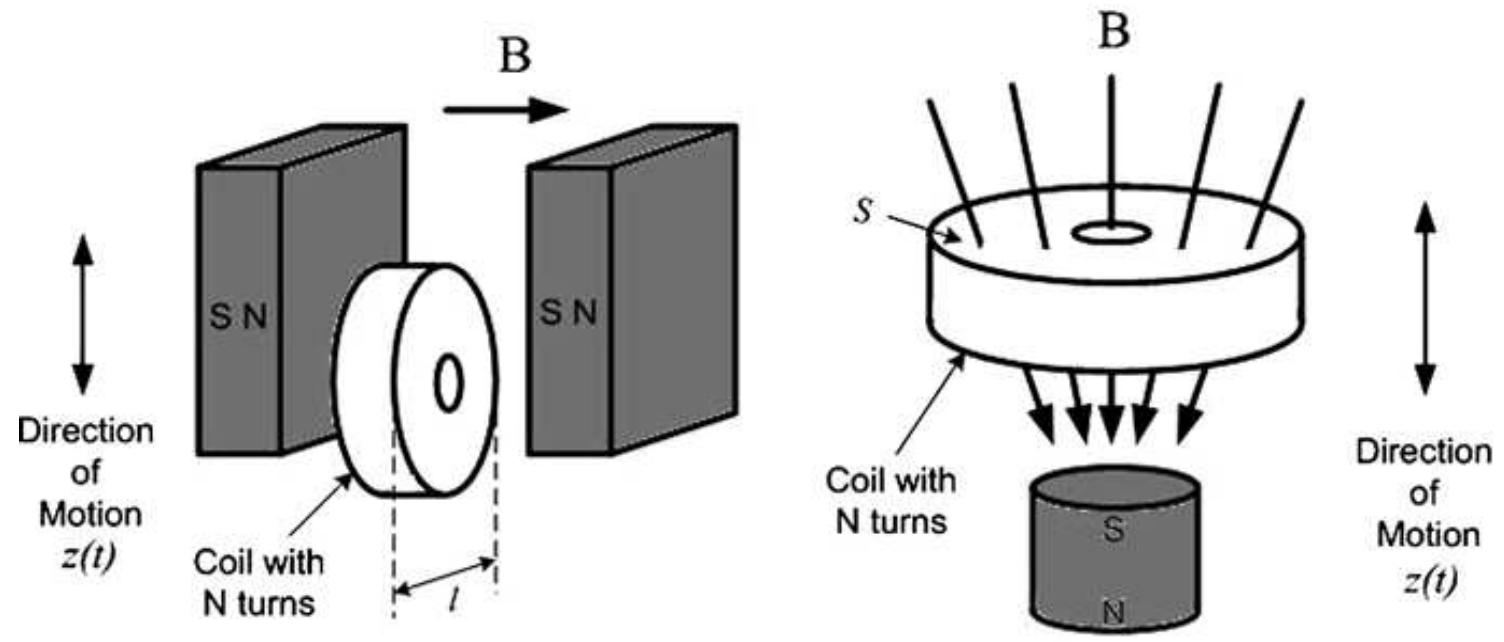

Figure 1.5. General prototypes of electromagnetic energy harvester [40].

proof mass moving through the magnetic flux from a permanent magnet (Figure 1.5). The induced voltage across the coil is proportional to the strength of the magnetic field, the velocity of the relative motion and the number of turns of the coil. 
A variety of MEMS electromagnetic energy harvesters were reported in the past years. For examples, Wang et al. developed an electromagnetic energy harvester for harvesting energy from vibration induced by Kármán vortex street. The device converts flow energy into electrical energy by fluid flow, vortex shedding from a bluff body and electromagnetic induction (Figure 1.6(a)). A power of $1.77 \mu \mathrm{W}$ at $0.3 \mathrm{kPa}, 62 \mathrm{~Hz}$ and $37.9 \mathrm{~cm}^{3}$ was reported [56]. P. Wang et al. designed an electromagnetic vibration harvester with sandwiched structure and air channel which consists of a top coil, a bottom coil, an NdFeB permanent magnet and a nickel planar spring integrated with silicon frame (Figure 1.6(b)). The load voltage generated by the prototype is $142.5 \mathrm{mV}$ when the prototype is at resonance of $280 \mathrm{~Hz}$ and under excitation acceleration of $10 \mathrm{~m} / \mathrm{s}^{2}$. The maximal load power obtained is about $17.2 \mu \mathrm{W}$ at load resistance of $81 \Omega$. [57]. Beeby et al. developed an electromagnetic energy harvester using four magnets arranged on an etched cantilever with a wound coil located within the moving magnetic field. The device reported an average power of $46 \mu \mathrm{W}$ under an excitation with amplitude of $0.589 \mathrm{~m} / \mathrm{s}^{2}$ and a frequency of $52 \mathrm{~Hz}$ with the prototype having a volume of $150 \mathrm{~mm}^{3}$ and a mass of $0.66 \mathrm{~g}$ [58]. Ashraf et al. showed a bounded vibration energy harvester whereby mechanical stoppers have been used in order to confine the proof mass movement within the elastic limits of the spring and geometrical limits of the device. Results showed that at a frequency of $10 \mathrm{~Hz}$ with a peak acceleration of $1 \mathrm{~g}$, the harvester responds at a higher frequency of $20 \mathrm{~Hz}$ and gives a peak power of $2.68 \mathrm{~mW}$ and a peak to peak voltage of $2.62 \mathrm{~V}$ across a load of $220 \Omega$. The average power density of $65.74 \mu \mathrm{W} \mathrm{cm} \mathrm{cm}^{-3}$ obtained at $10 \mathrm{~Hz}$ and $1 \mathrm{~g}$ excitation monotonically increases with frequency up to $341.86 \mu \mathrm{W} \mathrm{cm} \mathrm{cm}^{-3}$ at $18 \mathrm{~Hz}$ [59]. 
(a)

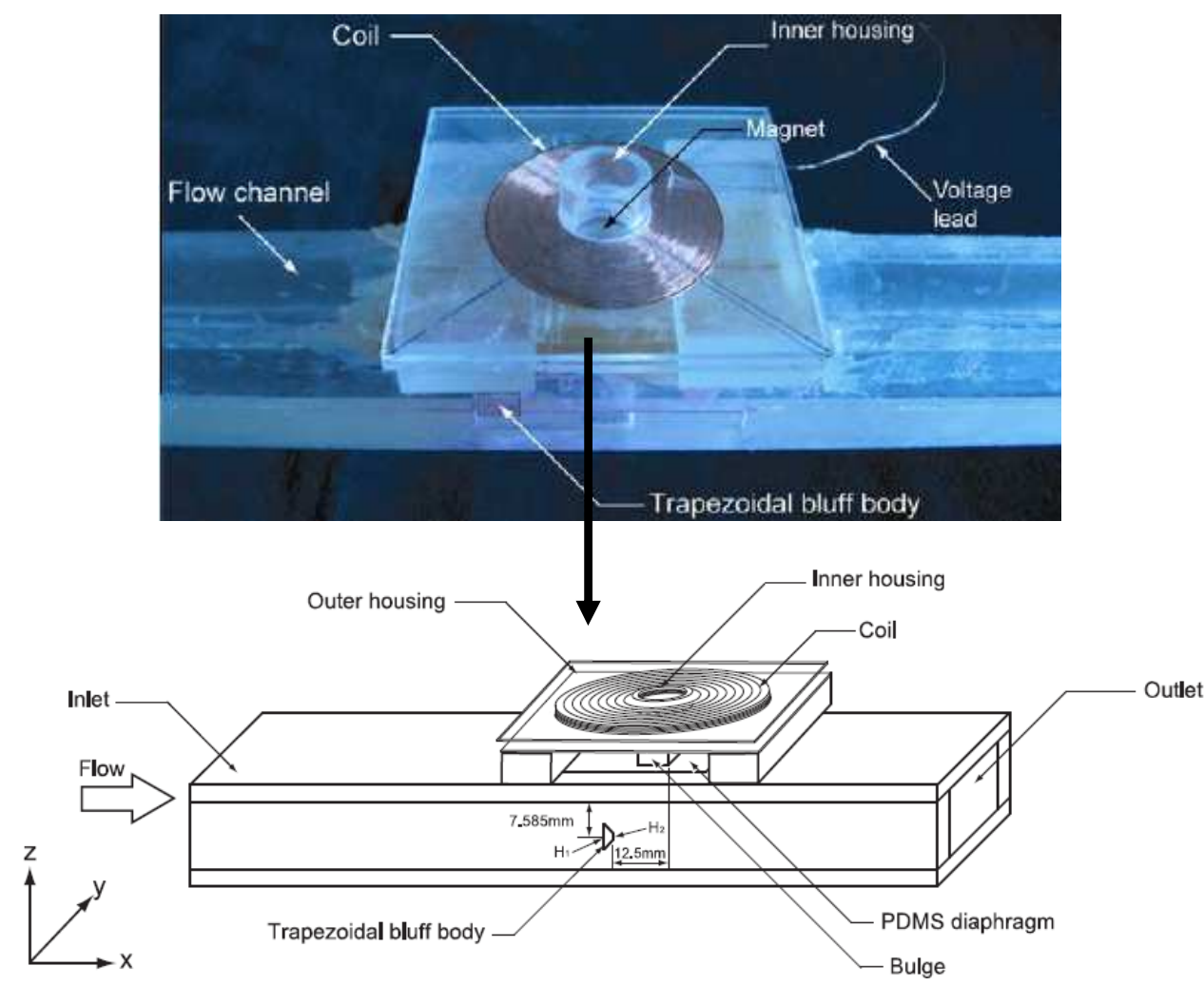

(b)

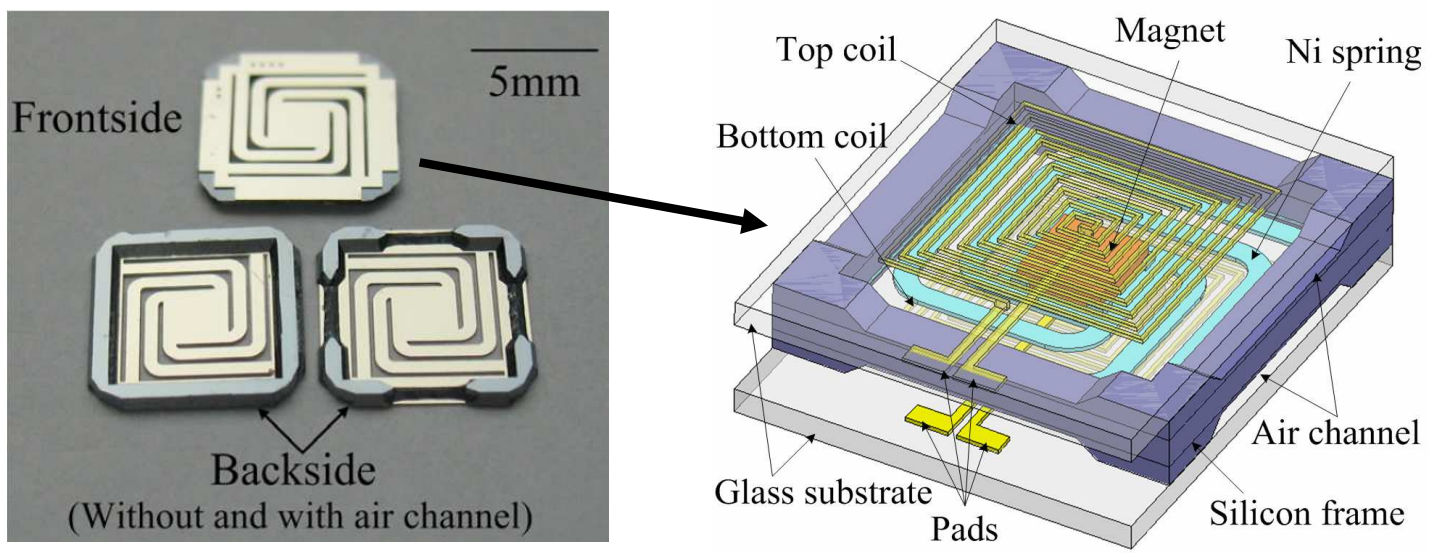

Figure 1.6. Several electromagnetic MEMS energy harvesters developed by other research groups, (a) an electromagnetic energy harvester [56], (b) a sandwiched electromagnetic energy harvester [57].

It should be noted that many of applications using MEMS electromagnetic energy harvester were adapted in macro scale systems because the achievements of gain- 
ing sufficient forces for low frequency applications is still challenging in micro dimensions $[60,61]$ and the integration of the bulky parts in such devices with CMOS is costly. In addition, MEMS with electroplated coils and magnets may not be able to produce useful power levels due to poor electromagnetic coupling, and the micromachined electromagnetic harvesters tend to result in low output voltages making rectification difficult due to the limited number of coil turns [61].

The piezoelectric effect is the ability of some materials to generate an electric potential in response to an applied mechanical stress. The electrical polarization is proportional to the applied strain. The piezoelectric mechanism of power harvesting utilizes strains caused by mechanical vibrations in a piezoelectric material inducing a charge separation across the material, therefore producing a voltage (see Figure 1.7(a) and 1.7(b)). Piezoelectric energy harvesters typically work in either 33 mode or 31 mode.
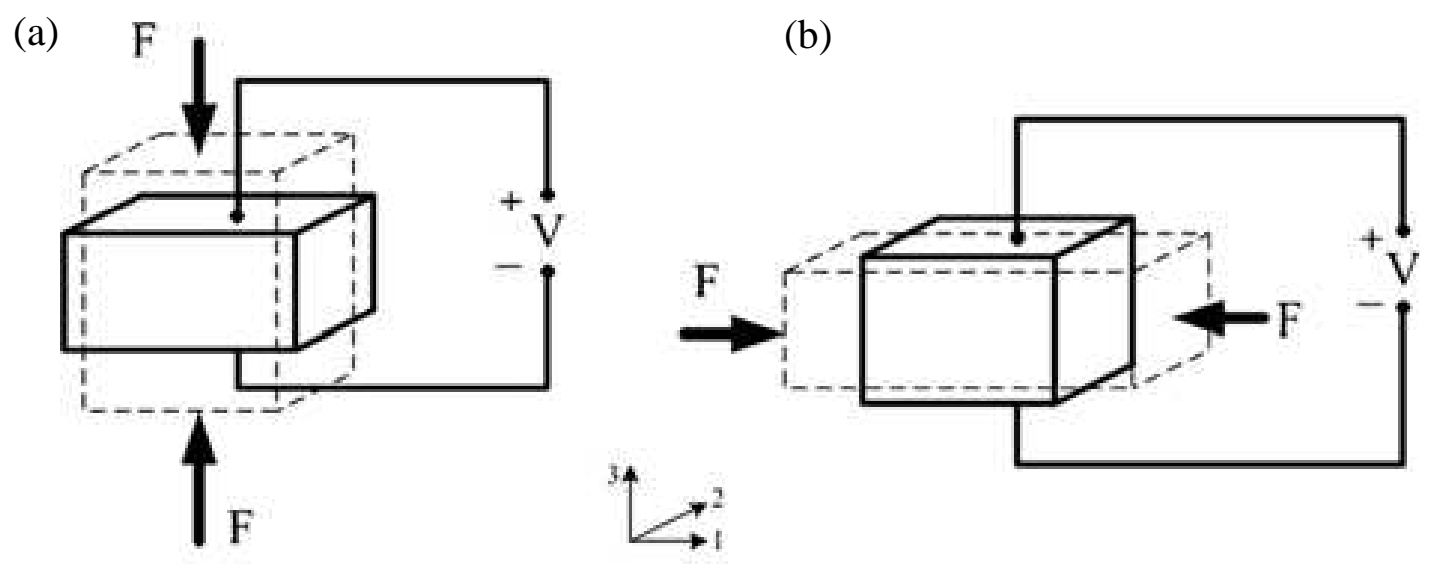

Figure 1.7. The piezoelectric effect: voltage induced by mechanical strains in piezoelectric material. (a) 33 mode, (b) 31mode [40].

In the 33 mode, a force is applied in the same direction as the poling direction, such as the compression of a piezoelectric block that has electrodes on its top and bottom surfaces. In the 31 mode, a lateral force is applied in the direction perpendicular to the 
poling direction, a bending beam that has electrodes on its top and bottom surfaces. Generally, the 31 mode has been the most commonly used coupling mode although the 31 mode has a lower coupling coefficient than the 33 mode [40, 62].

Implementation of piezoelectric power generation requires a piezoelectric material, usually a ceramic such as Lead Zirconium Titanate (PZT) in monolith or thin film form. The latter can be incorporated into a MEMS device. Damping forces of piezoelectric material tend to be small so that the piezoelectric harvesters are suited to resonant devices. Piezoelectric devices have been investigated extensively. Several research groups have recently proposed wideband piezoelectric harvester [63-69]. Many of reported piezoelectric transducers have overcome the limitation to low frequencies application [64-68], and have achieved reasonable high voltage levels. For examples, $\mathrm{Xu}$ et al. reported a piezoelectric energy harvester consisting of cantilever beam and cymbal transducers based on piezoelectric single crystal (Figure 1.8(a)). Under an acceleration of $3.2 \mathrm{~g}$, a peak voltage of $38 \mathrm{~V}$, a maximum power of $3.7 \mathrm{~mW}$ were reported at $102 \mathrm{~Hz}$ with a proof mass of $4.2 \mathrm{~g}$ [66]. Berdy et al. presented a low-frequency piezoelectric vibration energy harvester consists of a laser-machined meandering PZT bimorph for sensor node applications (Figure 1.8(b)). The energy harvester was reported with the measured power output and normalized power density of $118 \mu \mathrm{W}$ and $5.02 \mu \mathrm{W} / \mathrm{mm}^{3} / \mathrm{g}^{2}$, respectively, when excited by an acceleration magnitude of $0.2 \mathrm{~g}$ at $49.7 \mathrm{~Hz}$ [67]. Liu et al. reported a MEMS piezoelectric energy harvesting cantilever with low resonant frequency and wide operation bandwidth which consists of a silicon beam integrated with PZT elements parallel arranged on top and a silicon proof mass resulting in a low resonant frequency of $36 \mathrm{~Hz}$. The device shows steadily increased power generation from 
(a)

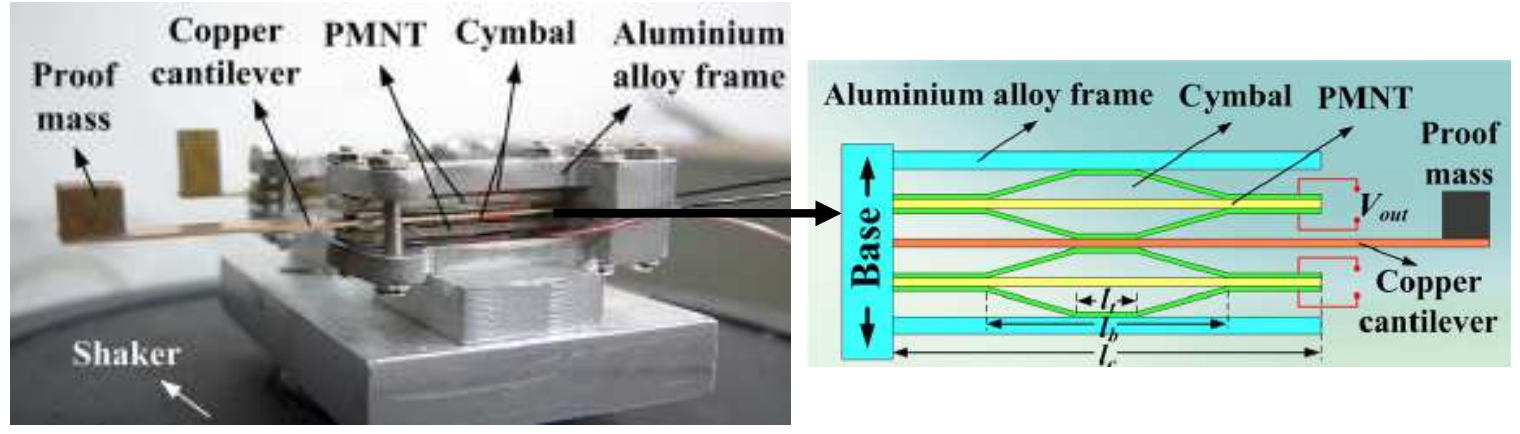

(b)

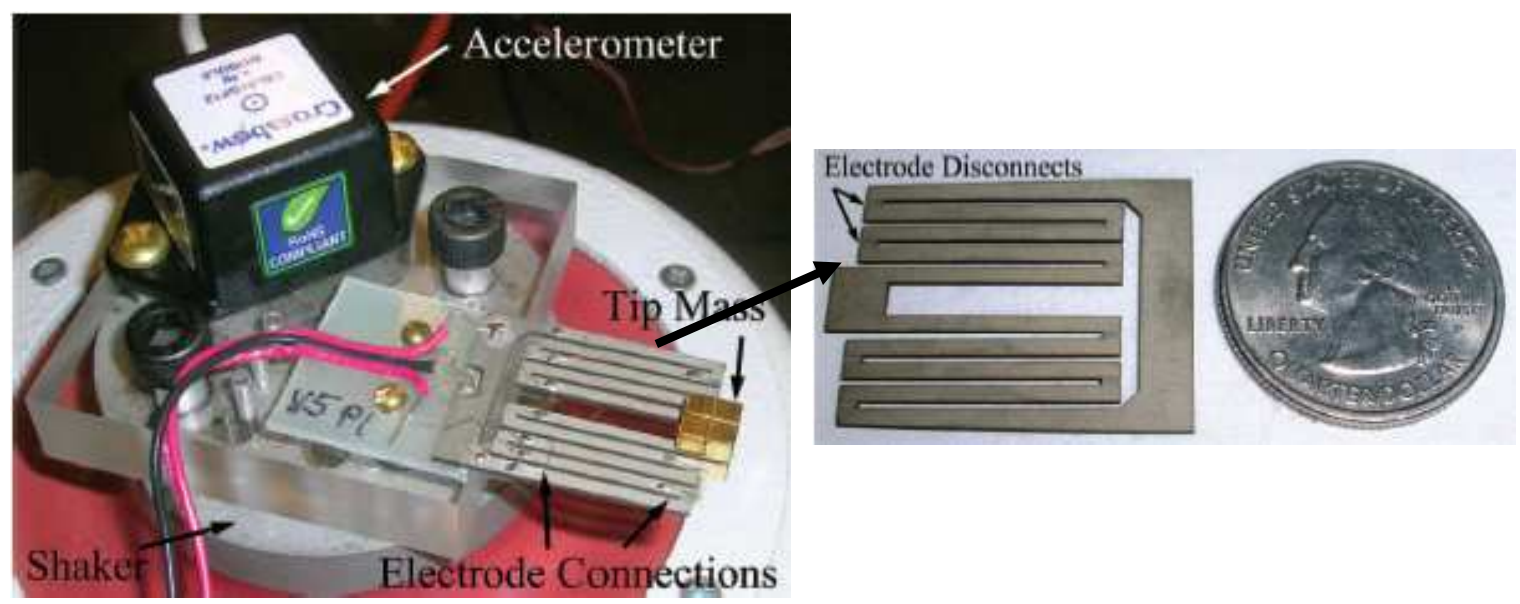

Figure 1.8. Various piezoelectic vibration energy harvester reported in recent years, (a) a low frequency energy harvester with piezoelectric cantilever reported by Xu et al. [66], (b) a low-frequency meandering piezoelectric vibration energy harvester presented by Berdy et al. [67].

$19.4 \mathrm{nW}$ to $51.3 \mathrm{nW}$ within the operation frequency bandwidth ranging from $30 \mathrm{~Hz}$ to 47 $\mathrm{Hz}$ at $1.0 \mathrm{~g}$ [68]. Hajati et al reported an ultra-wide bandwidth energy harvester by exploiting the nonlinear stiffness of a doubly clamped MEMS resonator. The main harvester part consists of four doubly clamped beams before it is assembled to an external proof mass. The device achieved a power density up to $2 \mathrm{~W} / \mathrm{cm}^{3}$ [69].

Piezoelectric energy harvesters have the simplest structure among the three trans- 
duction mechanisms and they can produce appropriate voltages for electronic devices. However, the mechanical properties of the piezoelectric material may limit overall performance and lifespan of the harvester. The output impedance of the piezo element is dominated by its capacitance, which due to its small size cannot be tuned out with a realistic inductance at the frequencies of interest. Although piezoelectric thin films can be integrated into a MEMS fabrication process, the piezoelectric coupling is greatly reduced [40]. Therefore, the potential for integration with microelectronics is less than that for electrostatic energy harvester which will be presented in the next section.

\subsection{MEMS electrostatic energy harvester}

The fundamental concept of electrostatic energy harvester is to take advantage of capacitance variance from variable capacitor which can be fabricated with MEMS micromachining technology. The device is driven by mechanical vibrations and oscillates between a maximum capacitance and a minimum capacitance. If the charge on the capacitor is conserved, the voltage will increase as the capacitance decreases or decrease as capacitance increases. If the voltage across the capacitor is constrained, the charge will move from the capacitor to a storage capacitor or to a resistive load as the capacitance decreases. In either case, mechanical kinetic energy is converted to electrical energy. Meninger et. al. gave a good explanation and comparison for the effectiveness between the charge constrained conversion and voltage constrained conversion [70]. The reported electrostatic transducer by far typically uses one of three basic topologies for micromachined variable capacitors, which are in-plane overlap, in-plane gap closing and out-of-plane gap closing (Figure 1.9) [40] [71]. The device shown in Figure 1.9(a) is referred to as an in-plane overlap transducer since the change in capacitance arises from 
(a)

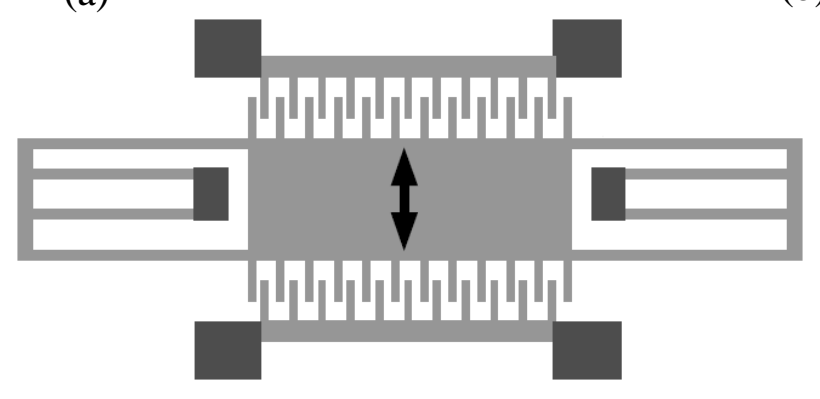

(b)

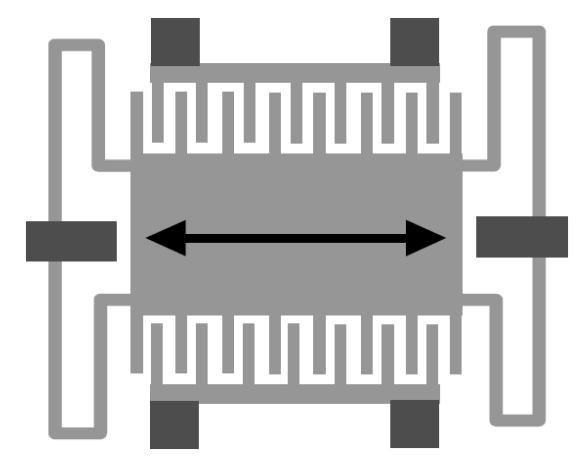

(c)

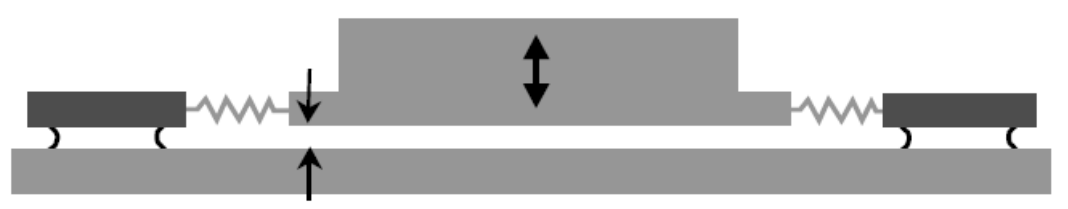

Figure 1.9. Electrostatic generators using three different topologies micromachined variable capacitors, (a) in-plane overlap, (b) in-plane gap closing, and (c) out-of-plane gap closing [71].

the changing overlap area of the interdigitated fingers. As the center plate moves in the direction shown, the overlap area, and thus the capacitance of the fingers oscillates. Figure 1.9(b) is referred to as an in-plane gap closing transducer because the capacitance changes due to the changes of the dielectric gap between the interdigitated fingers. The device shown in Figure 1.9(c) is referred to as an out-of-plane gap closing transducer shown. It oscillates out of the plane of the wafer, and changes its capacitance by changing the dielectric distance between two parallel plates [71].

Although the achievable energy density is usually lower than that of piezoelectric and electromagnetic generators, it is CMOS compatible which allows the integration of electronic circuits and MEMS on the same chip. This integration will lead to the production of low cost arrays of energy sources. In the past decade, the electrostatic energy harvester uses various designs were reported [72-79]. A vast majority of 
electrostatic devices were designed and fabricated with multi-gap comb fingers microstructure [74, 80] (see Figure 1.10(a) and 1.10(b)) or bulk micromachined proof mass suspended within a frame above the substrate [81, 82] (Figure 1.10(c)) for energy scavenging and voltage step-up conversion. It should be noted that most reported electrostatic energy harvester use bulk micromachining in which the proof mass can be formed from the whole thickness of the wafer. One disadvantage of electrostatic energy harvesters is that the devices always require a precharge voltage (supplied by external DC

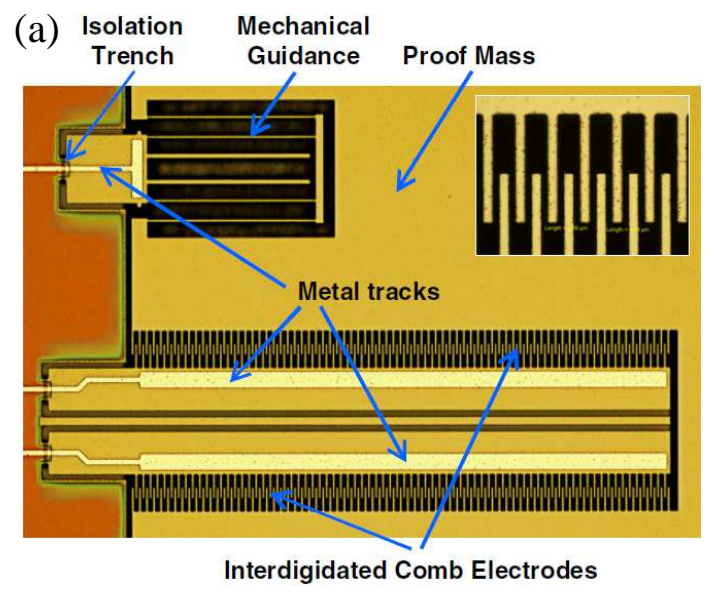

(b)

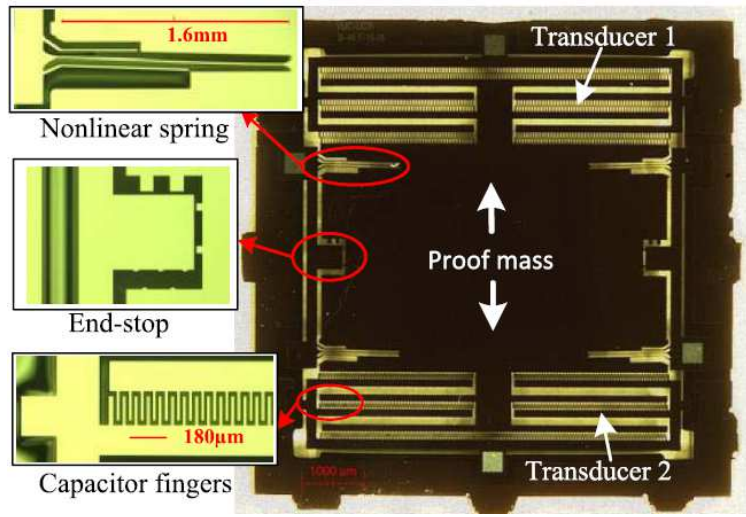

(c)
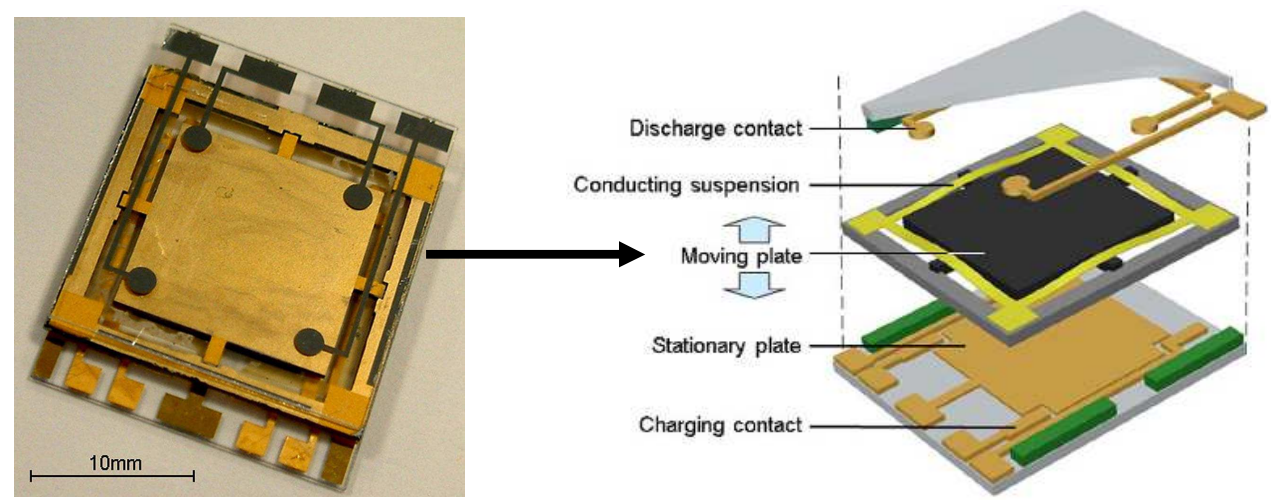

Figure 1.10. In-plane and out-of-plane electrostatic energy harvesters, (a) an in-plane MEMS electrostatic transducer using interdigitated comb fingers structure [74], (b) nonlinear curved springs and comb fingers designed for an in-plane MEMS electrostatic energy harvester [80], (c) a low frequency out-of-plane MEMS electrostatic energy harvester using a Si proof mass suspended with polyimide [81, 82]. 
bias) to operate. The pre-charging issue can be resolved by integrating an electret layer into the MEMS energy harvester. Electret is the electrostatic analog of a magnet, which has a quasi-permanent electric charge or dipole polarization. Electret can be charged using corona discharging system, and backlighted thyraton techniques [83-85].

The most recent reported output power from various electrostatic energy harvesters ranged from $\mathrm{nW}$ to several $\mu \mathrm{W}$. For examples, Nguyen et al. developed a MEMS electrostatic energy harvester with nonlinear springs to widen the bandwidth of frequency responses (Figure 1.11(a)). The device can generate a power of $85 \mathrm{nW}$ at 560 $\mathrm{Hz}$ using a peak amplitude of $0.14 \mathrm{~g}$ and bias voltage of $28.4 \mathrm{~V}$ [80, 89]. Suzuki et al. reported an in-plane vibration-driven MEMS electret energy harvester with a proof mass supported by polymer springs and counter electrodes (Figure 1.11(b)). The device generated power up to $6 \mu \mathrm{W}$ from $40 \mathrm{~Hz}$ vibration. Their another prototype using similar topology of design reported a power of $1.5 \mu \mathrm{W}$ at a frequency range of $16-28 \mathrm{~Hz}$ using a suspension mass of $10.2 \times 11.6 \mathrm{~mm}^{2}$ at $0.5 \mathrm{~g}$ acceleration [86-88]. Cuong and Halvorsen et al. reported electrostatic energy harvesting prototypes with integrated multi-transducer design which involve a reference master transducer with end-stops and an impact slave transducer with movable end-stops (Figure 1.11(c)). The device produced a power of $81.2 \mathrm{nW}$ at $702 \mathrm{~Hz}$ using active die dimension $4 \times 5 \mathrm{~mm}^{2}$ at $2.7 \mathrm{~g}$ acceleration $[90,91]$.

We have chosen to work with a MEMS electrostatic energy harvester with out-ofplane gap closing variable capacitor microstructure fabricated using surface micromachining technology. Since the capacitance is proportional to the overlap area and inversely proportional to the gap of two parallel plates in out-of-plane gap closing variable capacitor, relative displacement of the plates leads to the capacitance change. In 
(a)

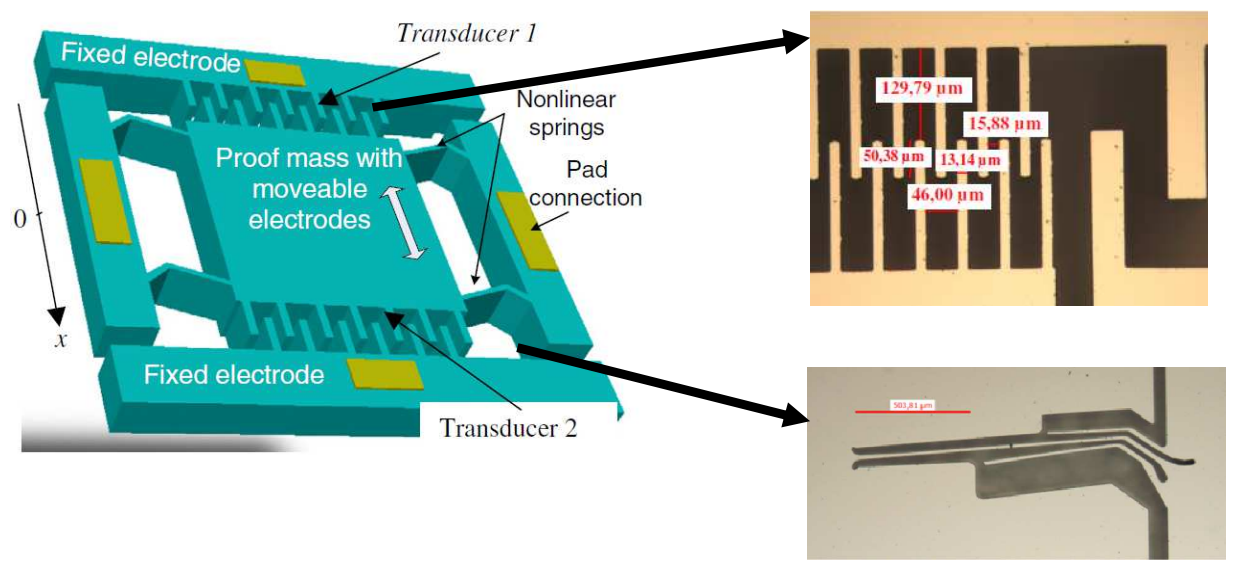

(b)

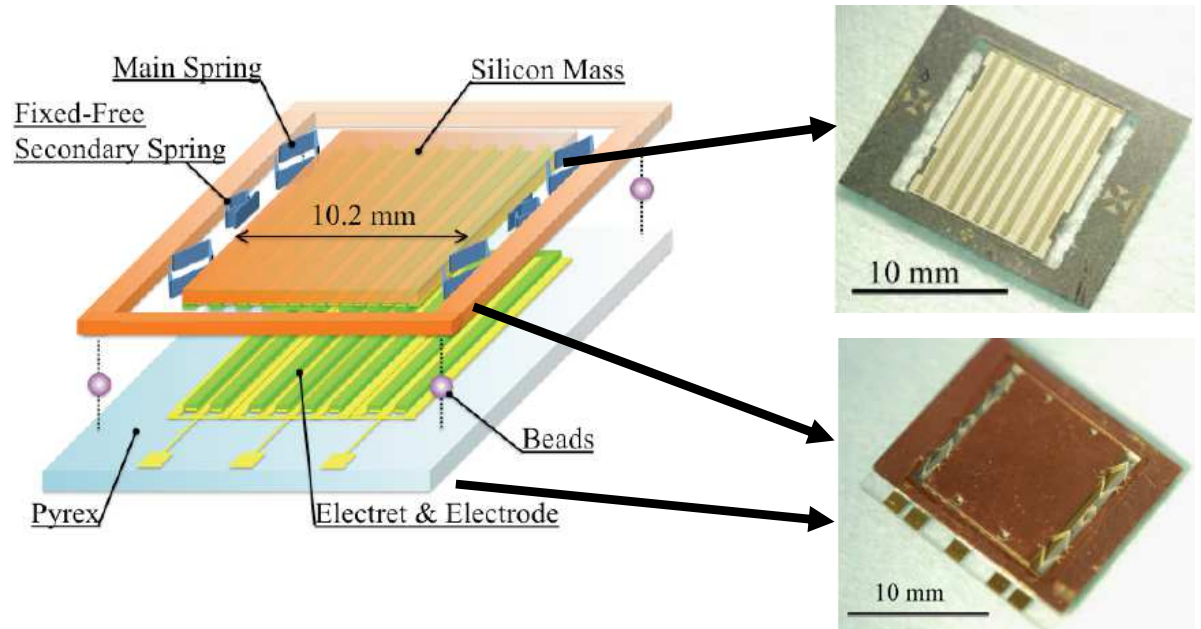

(c)

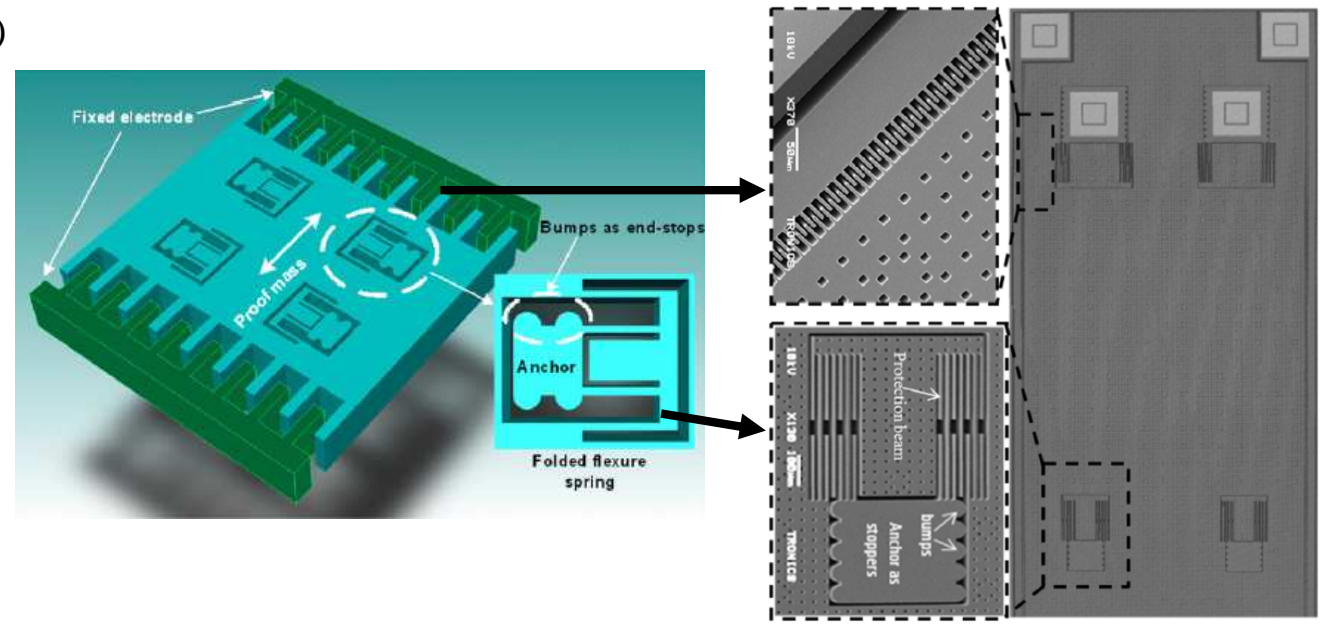

Figure 1.11. The most recent reported prototypes of electrostatic MEMS energy harvesters with, (a) nonlinear springs developed by Nguyen et al. [80, 89], (b) an in-plane vibration-driven using electret layer reported by Suzuki et al. [86-88], (c) an integrated electrostatic multi-transducer presented by Cuong and Halvorsen et al. [90, 91]. 
the next chapter, the design and operation principle of our novel MEMS electrostatic energy harvester will described be in details. 


\section{CHAPTER 2}

\section{DESIGN AND THEORY}

This chapter first presents the design of our MEMS variable capacitor with novel structure of two interactive variable capacitive cavities. The design includes using single and array of MEMS variable capacitor for energy harvesting. The chapter will then provide the theoretical background of power harvesting using parallel plate variable capacitor. In addition, the operation principle of the device will be described in details.

\subsection{Designs of the MEMS variable capacitor with dual-cavity for power harvesting}

The MEMS power harvester, an out-of-plane gap closing variable capacitor, is designed using parallel plate structure forming two interactive capacitive cavities connected in series. When the capacitance increases for one cavity, it will decrease for the other. This allows us to use both capacitors to generate power and optimize the efficiency of power harvesting.

\subsubsection{Design 1: Single MEMS variable capacitor with two-cavity}

The first design of MEMS electrostatic energy harvester consists of a single variable capacitor having a movable proof mass plate with an area of $2 \times 2 \mathrm{~mm}^{2}$ suspended between two fixed plates on the top and bottom silicon substrates with two air gaps in between, forming two separate interactive vertical capacitors (Figure 2.1(a) and 2.1(b)). The movable plate is designed to be perforated (Figure 2.2(a)) in order to reduce the squeeze film air damping and help to release the photoresist sacrificial layer during the 
final step of the fabrication process. The movable plate is supported by four serpentine springs that are attached to the address lines on a silicon substrate only at the anchors' points which are made of electroplated nickel (Ni). The stiffness of the serpentine suspension springs will determine the resonance frequency of the movable plate. The dimensions of designed perforated movable plate and serpentine springs are shown in Figure 2.2(a). Five mechanical stoppers, made of gold ( $\mathrm{Au})$, are electroplated on the fixed plates in order to prevent the pull-in behavior and modify the impact with the movable plate (see Figure 2.2(b)). Silicon oxide $\left(\mathrm{SiO}_{2}\right)$ and silicon nitrite $\left(\mathrm{Si}_{3} \mathrm{~N}_{4}\right)$ thin layers were deposited and patterned to insulate the fixed plates with stoppers and enhance the dielectric property of the capacitive cavities. In addition, they can be used as an electret

(a)

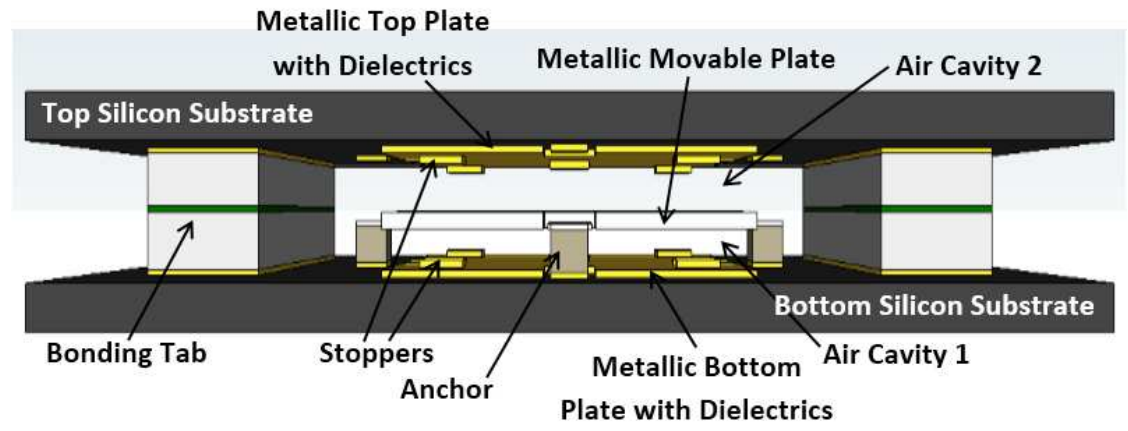

(b)

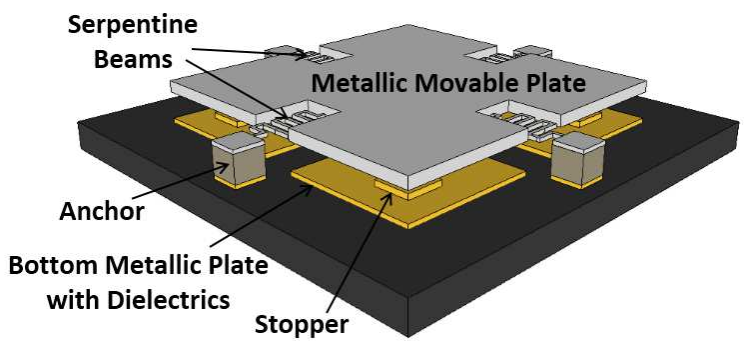

(c)

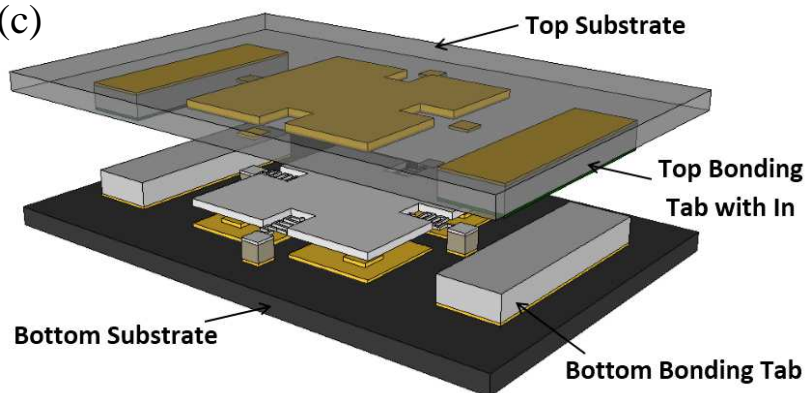

Figure 2.1. 3D Schematic of single MEMS variable capacitor with dual-cavity, (a) front view of the whole device with all components labeled, (b) structure of movable plate, (a) different angle view of the whole device, top substrate is risen up and made transparent for show. 


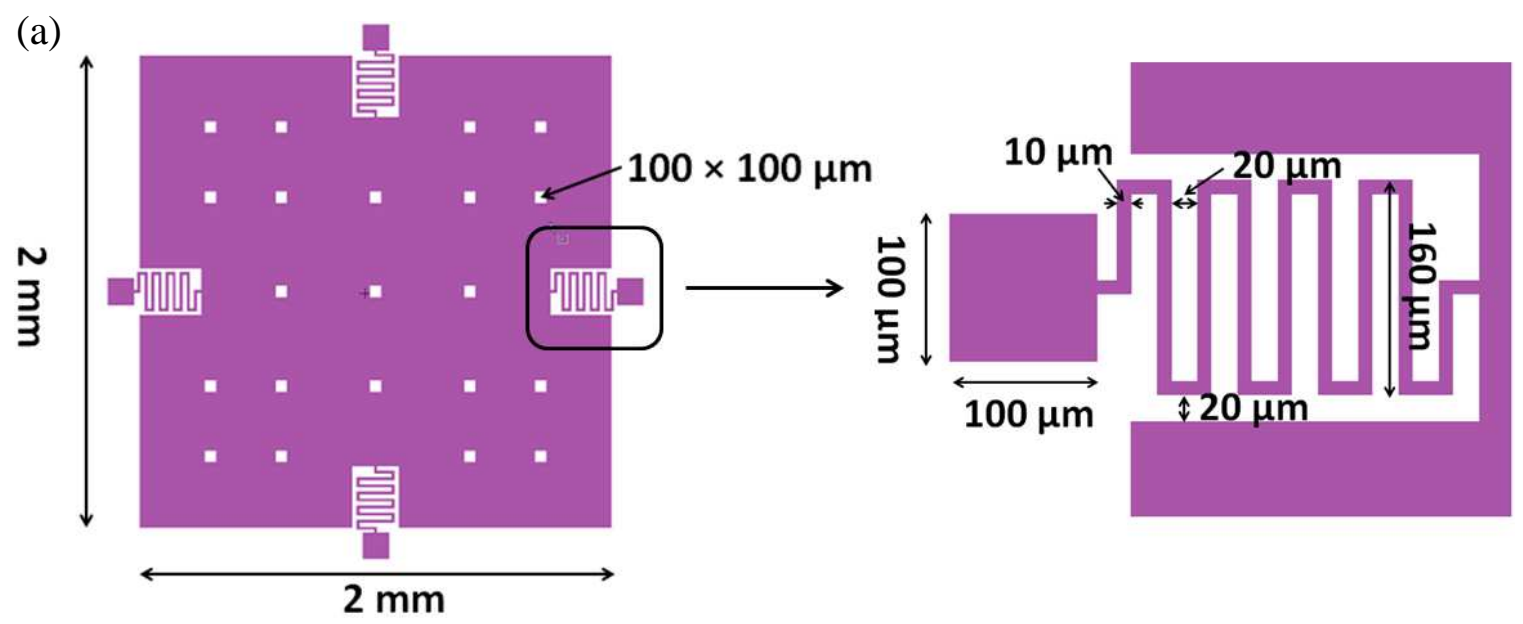

(b)

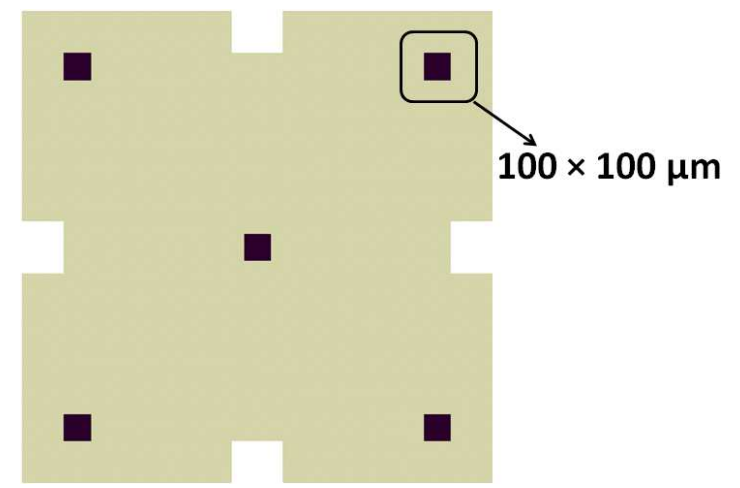

Figure 2.2. (a) Dimensions of the perforated movable plate and serpentine suspension beams, (b) Dimension of the mechanical stoppers on the fixed plates.

to trap charges at the interface between the two layers to create polarization voltage in the capacitive cavities using Corona charging method in our future process.

The second air cavity is created by bonding a second silicon substrate onto the first one. The second substrate contains patterned fixed plates, $\mathrm{SiO}_{2} / \mathrm{Si}_{3} \mathrm{~N}_{4}$ dielectric layer, and two strips of bonding tabs made of electroplated Ni and thin layer of Indium (In) for wafer bonding at low temperature and to provide electrical contacts to the electrodes. The strip bonding tabs on both substrates are aligned and bonded together when In layer is softened under relatively low temperature without damaging the integrity of the device (see Figure 2.1(c)). The proof mass plates, serpentine suspension beams, anchors and bonding tabs are all made of electroplated $\mathrm{Ni}$ which has been extensively studied as a 
mechanically durable material with controllable residual stress [92]. The details of device dimensions used in the fabrication of design 1 are listed in Table 2.

Table 2. The device dimensions used in the fabrication for design 1.

\begin{tabular}{|l|c|c|c|}
\hline & Width $(\boldsymbol{\mu m})$ & Length $(\boldsymbol{\mu m})$ & $\begin{array}{c}\text { Thickness/Height } \\
(\boldsymbol{\mu m})\end{array}$ \\
\hline Movable plate & 2000 & 2000 & 30 and 40 \\
\hline Top and bottom plates & 2000 & 2000 & 0.25 \\
\hline Serpentine beams & 15 & 1485 & 3 and 5 \\
\hline Anchors & 100 & 100 & 30 \\
\hline Stopper & 100 & 100 & 2 and 4 \\
\hline Bonding tabs & 2000 & 3500 & 30 \\
\hline Air gaps & & & 30 \\
\hline
\end{tabular}

\subsubsection{Design 2: MEMS variable capacitor array with two-cavity}

In order to increase the total harvested energy, the four capacitors in a linear array with the same design that was described in the previous section were connected in parallel (Figure 2.3). The charge flow generated by array of capacitors is theoretically expected to be 4 times higher than that of a single capacitor due to its higher variation of capacitance. Since energy harvesting is directly proportional to the generation of charge flow, the total output power from the $1 \times 4$ array of capacitors is expected to be 4 times bigger than that of a single capacitor. Similar as design 1, the top air cavity of the capacitors is created by bonding a second silicon substrate with 4 fixed plates with 
$\mathrm{SiO}_{2} / \mathrm{Si}_{3} \mathrm{~N}_{4}$ dielectric layer at locations corresponding to each capacitor onto the bottom substrate. Two long strips of bonding tabs on the top substrate are made of electroplated Ni with another thin layer of In electroplated on its top for wafer bonding and to provide electrical contacts to the electrodes. The long strip bonding tabs on both substrates are aligned and bonded together when In layer is softened under relatively low temperature without damaging the integrity of the whole device structure. The details of the device dimensions used in the fabrication for design 2 are listed in Table 3.

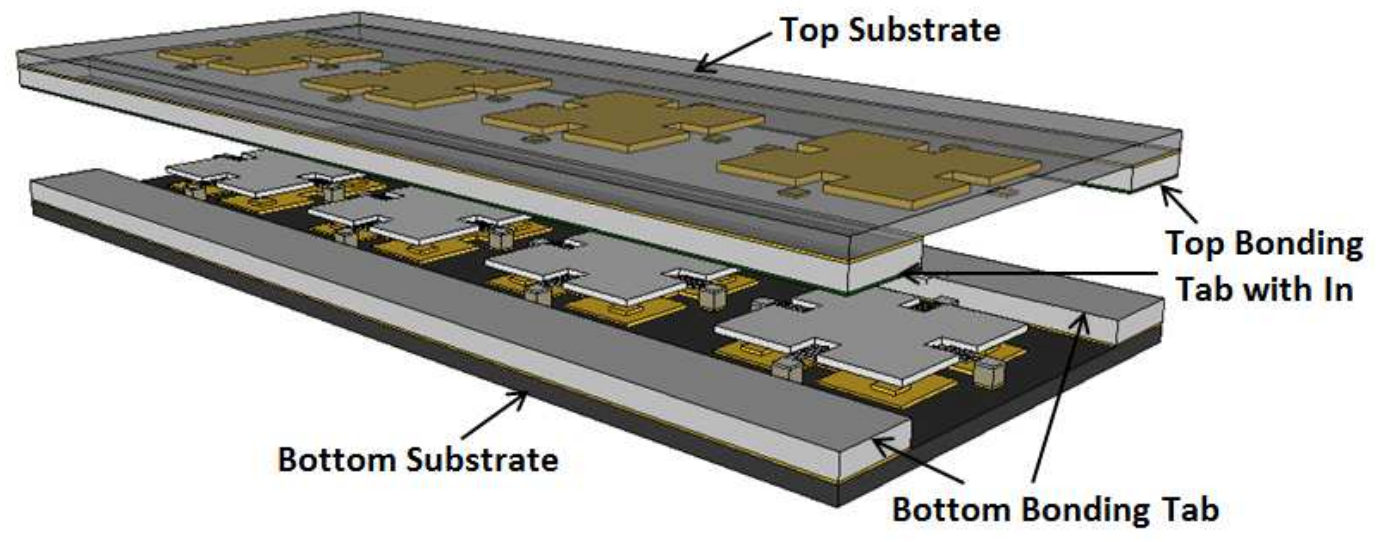

Figure 2.3. A 3D Schematic of MEMS variable capacitor array with dual-cavity, top substrate is risen up and made transparent for show.

The majority of reported MEMS electrostatic energy harvesters are mechanically designed as a resonator with a very narrow bandwidth near their corresponding resonance frequencies $[74,75,81,82]$. However, in the real environment, the ambient vibration is more often random with power spread over a frequency bandwidth. Another advantage of using an array of resonant variable capacitors is that each capacitor can be designed with different resonance frequency in order to increase the operational frequency range of the MEMS electrostatic energy harvester. However, such array energy harvesters have turned out with lower energy efficiency than that of single devices [93-95]. 
Table 3. The device dimensions used in the fabrication for design 2.

\begin{tabular}{|l|c|c|c|}
\hline & Width $(\boldsymbol{\mu m})$ & Length $(\boldsymbol{\mu m})$ & $\begin{array}{c}\text { Thickness/Height } \\
(\boldsymbol{\mu m})\end{array}$ \\
\hline Movable plate & 2000 & 2000 & 30 and 40 \\
\hline Top and bottom plates & 2000 & 2000 & 0.25 \\
\hline Serpentine beams & 15 & 1485 & 3 and 5 \\
\hline Anchors & 100 & 100 & 30 \\
\hline Stopper & 100 & 100 & 2 and 4 \\
\hline Bonding tabs & 2000 & 1340 & 30 \\
\hline Air gaps & \multicolumn{3}{|l}{} \\
\hline
\end{tabular}

\subsection{Theory of operation}

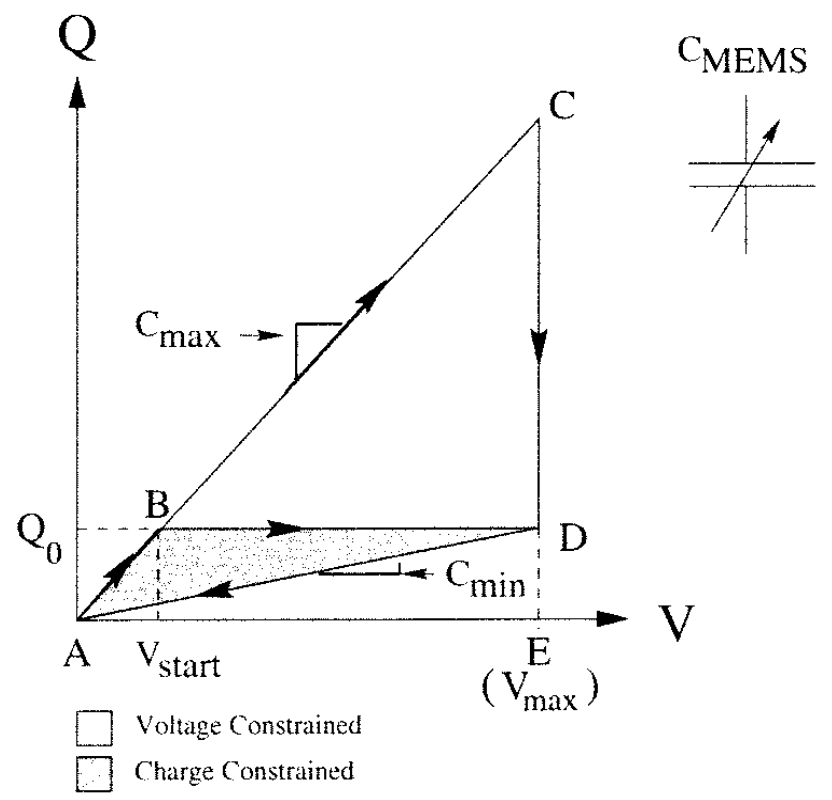

Figure 2.4. A charge-voltage plane of the variable capacitor for energy harvesting showing the charge-constrained and voltage constrained cycles [70]. 
We have mentioned in chapter 1 that the variable capacitor energy harvester can be operated either using charge-constrained conversion or voltage constrained conversion. Meninger et. al. gave a good explanation of the merits of charge constrained conversion and voltage constrained conversion [70]. As shown in Figure 2.4, there are two possible energy conversion cycles in the charge-voltage plane for the variable capacitor energy harvester. The path A-B-D-A depicts charge constrained conversion, while path A-C-D-A depicts voltage constrained conversion. The name of these two operation depicts which property is held constant during the conversion process while the other changes in response to a varying capacitance [70].

\subsubsection{Charge constrained conversion}

In the case of charge constrained conversion, capacitance of the MEMS variable capacitor, $\mathrm{C}_{\text {MEMS }}$ (see Figure 2.4), is initially charged to certain voltage level when its capacitance is at a maximum value, which corresponds to path segment A-B in Figure 2.4. As the capacitor vibrates, the voltage increases as capacitance decreases (because the total charge is conserved) until displacement of the movable plate reaches at a maximum position where $\mathrm{C}_{\mathrm{MEMS}}=\mathrm{C}_{\min }$ corresponding to point $\mathrm{D}$. The amount of charge initially injected into the capacitor was precalculated such that when $\mathrm{C}_{\text {MEMS }}$ reaches its minimum, the value of the voltage across the capacitor is $\mathrm{V}_{\max }$. During the transition from point $\mathrm{B}$ to D, the MEMS device is isolated with respect to the rest of the system so that no charge flow path exists, thus the total charge in the capacitor is conserved. Since the capacitor is forced to hold its charge, $\mathrm{V}$ must increase to satisfy $\mathrm{Q}=\mathrm{CV}$ as $\mathrm{C}$ decreases. The charge is then returned to a reservoir along path D-A. The net energy out is the shaded area ABDA. The net energy from charge constrained cycle $\left(E_{c c}\right)$ can be expressed as [70]: 


$$
E_{C C}=\frac{1}{2}\left(C_{\max }-C_{\min }\right) V_{\max } V_{\text {start }}
$$

\subsubsection{Voltage constrained conversion}

In the case of voltage constrained conversion, the cycle starts when the capacitor is charged up to maximum voltage, $\mathrm{V}_{\max }$, at its maximum capacitance $\mathrm{C}_{\max }$. During this time, $\mathrm{C}_{\text {MEMS }}$ is constant which corresponding to the straight line A-C. As the plate moves back, the capacitance decreases until the path C-D is traversed where the capacitance is at its minimum. We can see from the Figure 2.4 that the voltage is held constant during the path C-D in the conversion process. The mechanical vibration does work by causing charge to release from the capacitor into external reservoir which is presented by the path D-A. The charge on the capacitor then starts recovering (the path A-C) after capacitance reaches a minimum at $\mathrm{C}_{\mathrm{MEMS}}=\mathrm{C}_{\min }$. Therefore, the net energy $\mathrm{E}_{\mathrm{vc}}$ gained by voltage constrained conversion is the shaded area ACDA, which can be expressed as [70]:

$$
E_{V C}=\frac{1}{2}\left(C_{\max }-C_{\min }\right) V_{\max }^{2}
$$

It is obvious from the figure that the energy available from the charge constrained conversion is less than that available from the voltage constrained conversion by a factor of $\mathrm{V}_{\text {start }} / \mathrm{V}_{\max }$.

\subsubsection{Modified voltage constrained strategy}

To realize the same strategy either using charge constrained or voltage constrained conversion experimentally for our MEMS device, the experimental circuit must require complicated control system which involves extra electronic components introducing comparative high parasitic resistance and capacitance. Each capacitive cavity 
of our single MEMS capacitor is actually measured with capacitance about $100 \mathrm{pF}$ at its static state, and variation of its capacitance was estimated around $900 \mathrm{pF}$ when the plate was vibrating (initial gap at static state is $30 \mu \mathrm{m}$. If plate makes contacts with the stoppers as it vibrates, the gap can get to its minimum around 2-4 $\mu \mathrm{m}$ ). Thus, the charge flow induced by the changing of capacitance is considered small under regular bias DC below $15 \mathrm{~V}$. Therefore, it is difficult to characterize such small charge flow with the circuit with complicated control electronics since the parasitic resistance and capacitance can cause significant charge loss. Our experimental circuit simply uses high value load resistors (5M ohm in our case) to pull up the voltage which was then measured via unity amplifier when charge flows through the load resistors. In this way, it is easier for us to gain clear signals with minimum loss (see Figure 2.5).
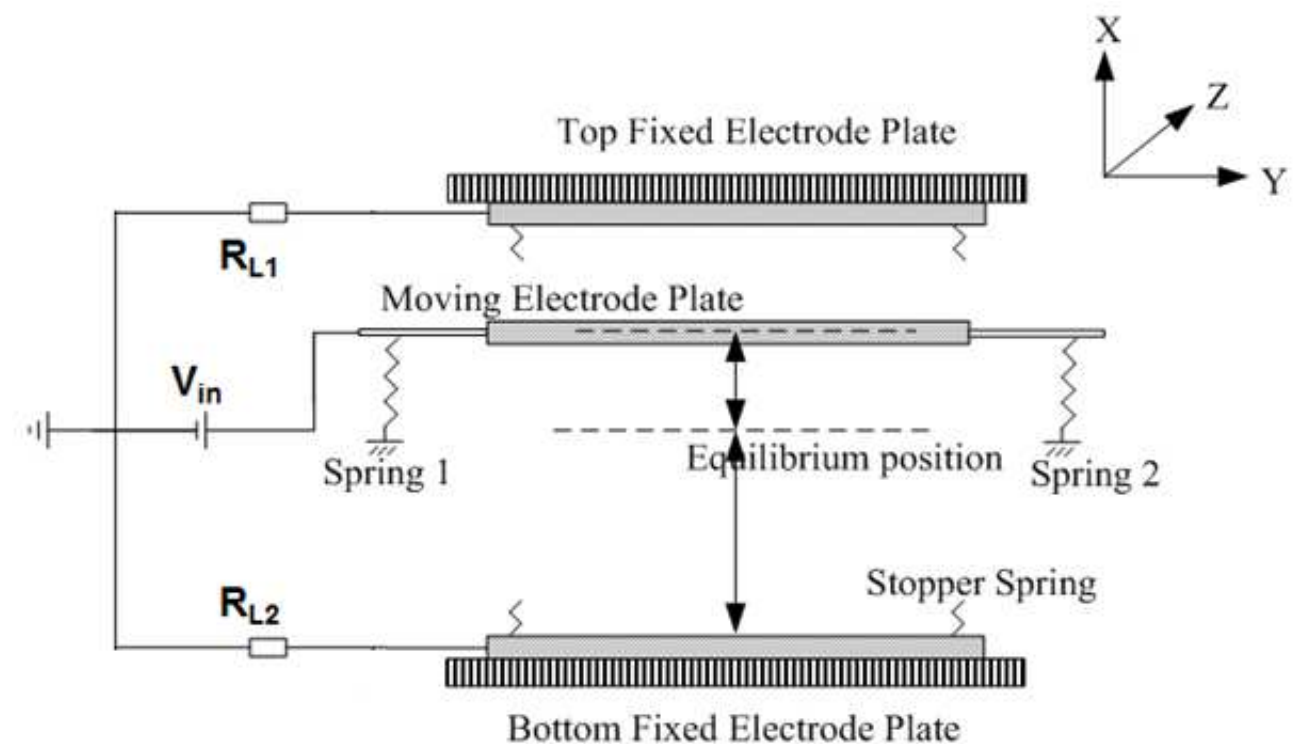

Figure 2.5. A schematic of operation principle for MEMS variable capacitor with twocavity.

Our MEMS capacitors energy harvester uses modified voltage constrain strategy for power harvesting and operates as follows: The top and bottom capacitors are initially 
charged by a low DC voltage source. When the plate moves down, capacitance of the bottom cavity increases and takes in charge from the low DC power source. Charging of bottom cavity stops when the plate reaches the lowest bottom position, then the plate tries to bounce back up so the gap of the bottom cavity increases and the gap of the top cavity decreases. Meanwhile, capacitance of the bottom cavity decreases and releases charge to corresponding load resistor, and capacitance of the top cavity starts increasing thereby taking charge from the low DC power source. Discharging of top cavity to its load resistor starts as its capacitance decreases when plate reaches the highest top position and then moves back down again. Consequently, two out-of-phase AC signals are generated from two capacitive cavities as above sequence repeats when the movable plate is vibrating. The details of device testing will be described in chapter 5 .

\subsection{Theoretical background of parallel plates variable capacitor}

The parallel-plate variable capacitor can be used as an energy harvester for direct vibration energy harvesting or as DC/DC converters. It has been shown that the energy converted is proportional to the capacitance range [70]. In DC/DC converters, the voltage step-up is proportional to the ratio of maximum to minimum capacitance. The capacitance is typically changed by one of the two geometric quantities: the overlap area and the gap. For either of the two configurations, the moving plate is subjected to forces from the elastic support, electrostatic forces between parallel plates and damping that are caused by the air surrounding the moving plate. If the chip containing the device is mounted on a vibrating body, the moving plate is also subjected to the inertia force (D'Alembert force) when viewed in a coordinate attached to the moving body. In

addition, both geometries are based on the mechanical displacements which are 
accommodated by the elasticity of the MEMS structure. The variable range is limited by the electromechanical coupling in the presence of an electric potential over the plates. The electrostatic attraction causes the gap to close by overcoming the resistance from the elastic support. When a constant voltage is applied, the capacitance increase causes more charges to be added and results in further closing of the gap. This positive feedback causes instability in parallel-plate actuators that limits the stable range of motion to $1 / 3$ of the gap.

When used as a DC/DC converter, the MEMS device must execute dynamic cycles of charging, capacitance change and discharge. The device often incorporates actuators that control the gap or the overlap area of the two capacitive plates. The position of the plates is determined by the forces acting on the plates. Mechanical and electrostatic forces act simultaneously in a dynamic fashion. When the device is used for power harvesting from mechanical vibration, the actuation to tune the capacitance may not be needed. Instead, the inertia force imposed on the device by the mechanical vibration serves to tune the capacitance.

\subsubsection{General model of parallel plates variable capacitor}

Available microfabrication techniques allow MEMS structure designs of parallel plate variable capacitor which simplify the mechanics models. The designs generally include elastic supports which allow displacement along one direction and plates which can be considered rigid (Figure 2.4). The system can be modeled using the following equation:

$$
m \ddot{x}+c \dot{x}+k x=f_{i}+f_{e}
$$


where $x$ is the plate displacement from the static equilibrium, $m$ the mass of the moving plate, and $k$ the stiffness of the elastic support. The coefficient $c$ accounts for the damping, mainly from the squeezed air film in the gap of the capacitive plates. The term $f_{i}$ represents the inertia force if the device is subjected to base excitation. In that case, the magnitude is simply the product of the plate mass and the base acceleration. The term $f_{e}$ represents the electrostatic force due to the charge on the plate.

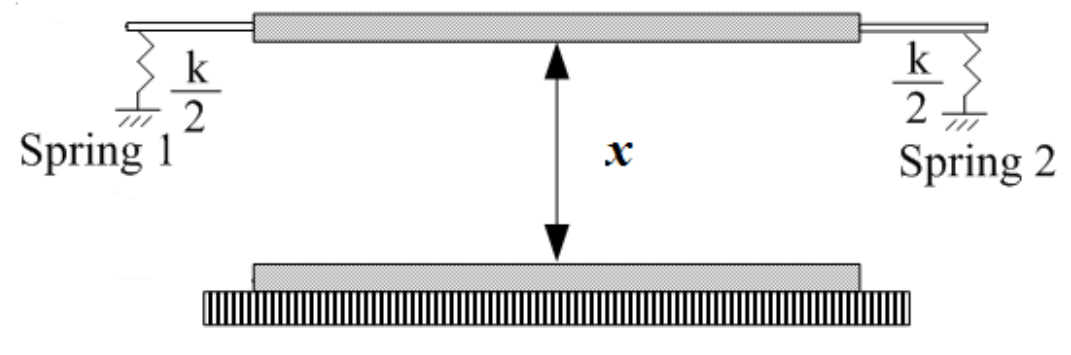

Figure 2.6. A schematic of the simplest model of parallel plate variable capacitor.

Our two-air-cavity design has two capacitors sharing a common electrode. The electrostatic force is the sum of the two. Through charging and discharging, the capacitive plates exchange energy with the electrical components both upstream and downstream; mechanical energy is dispensed in the process. If the device is sealed in a vacuum, the mechanical damping can be negligible. The electrostatic force $f_{e}$ refers to the electrostatic force over two capacitive plates. The plate capacitance is given by:

$$
C=\frac{\varepsilon A}{d}
$$

where $\varepsilon$ is electrical permittivity, $A$ the plate area, and $d$ the plate separation. For plates with varying overlap area, the electrostatic force caused by a voltage difference $V_{c}$, is:

$$
f_{e}=\frac{\varepsilon W V_{c}^{2}}{2 d}
$$


where $W$ is the width of the plates. In predominant interdigitated finger-type plates, the number of fingers has to be considered. Note that the electrostatic force is not dependent on the plate displacement $x$. For plates with varying gap, the electrostatic force caused by a voltage difference is:

$$
f_{e}=-\frac{\varepsilon A V_{c}^{2}}{2 d^{2}}
$$

Since the gap is varying, this force is dependent on the plate displacement $x$. When the gap decreases, the electrostatic force increases. This force is nonlinear, i.e. the increase is not proportional to the displacement. The increase may overwhelm the restoring force of the elastic support to cause the gap to collapse. When this is caused by the gradual increase of the voltage, a critical voltage, called the pull down voltage, is known to exist:

$$
V_{p d}=\sqrt{\frac{8 k d_{0}^{2}}{27 C_{0}}}
$$

where $d_{0}$ and $C_{0}$ are the gap and capacitance at static equilibrium when no voltage is applied.

\subsubsection{Model of electrical capacitance with dielectric layer}

In order to increase the harvested energy, we apply dielectric layer $\left(\mathrm{SiO}_{2} / \mathrm{Si}_{3} \mathrm{~N}_{4}\right)$ with high dielectric constant on the fixed top and bottom plates which is highly suitable for out-of-plane motion energy harvesting. The layer thickness is small compared to the separation between the fixed and the movable plates at equilibrium. Therefore, at equilibrium, the added layer has a negligible effect on the capacitance. However, when the moving plate touches the dielectric layer as permitted during normal operation, the capacitance becomes hundreds or even thousands of times bigger. 
With the dielectric layer, the capacitor is modeled as two capacitors in series (Figure 2.5(a)). This simple model assumes that the top surface of the dielectric layer is at equal electric potential. Therefore, we have a capacitor containing the air gap and a capacitor with a dielectric layer as medium. The relative dielectric constant of the dielectric layer is denoted by $\varepsilon_{r}$. The total capacitance between the moving electrode and the fixed electrode is given by equation (11):

(a)

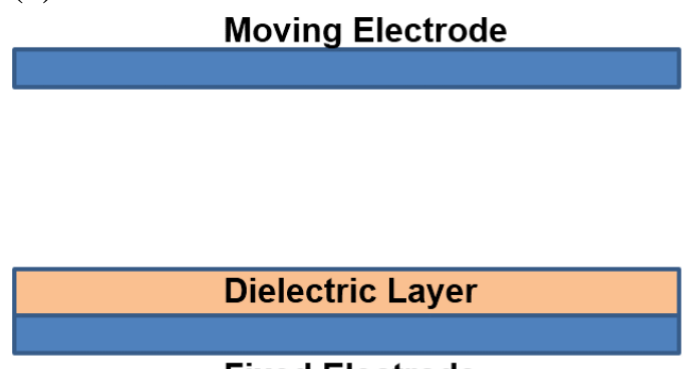

Fixed Electrode (b)
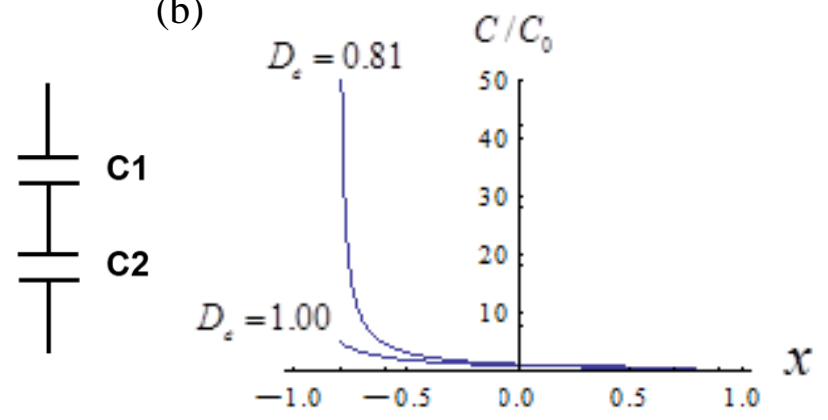

Figure 2.7. (a) The model capacitor incorporating the dielectric layer, (b) Capacitances as functions of the plate displacement. Purple: without dielectric layer. Blue: with dielectric layer and $\mathrm{De}=0.81$.

$$
\begin{gathered}
C=\frac{C_{1} C_{2}}{C_{1}+C_{2}} \longrightarrow C=C_{0} /\left(\frac{g_{d}}{\varepsilon_{r} d_{0}}+\frac{g_{\text {air }}}{d_{0}}\right) \\
\text { with } C_{1}=\frac{\mathcal{E}_{0} A}{g_{\text {air }}}, C_{2}=\frac{\mathcal{E}_{0} \varepsilon_{r} A}{g_{d}}, C_{0}=\frac{\mathcal{E}_{0} A}{d_{0}}
\end{gathered}
$$

where $A$ is the area of the electrodes, $\varepsilon_{0}$ and $\varepsilon_{r}$ are the permittivity of air, and the dielectric layer relative to vacuum, respectively. $g_{\text {air }}$ and $g_{d}$ are the air gap and the thickness of the dielectric layer respectively. In order to demonstrate the effect of the dielectric layer on the capacitance, we consider specifically the following design:

$$
g_{d}=0.2 d_{0}, g_{\text {air }}=(0.8+x) d_{0}, \varepsilon_{r}=1000
$$


Where $d_{0}$ is the separation of the two electrodes at equilibrium and $x$ is the dimensionless upward displacement of the moving electrode. Thus we get:

$$
C=C_{0} \frac{1}{0.0002+0.8+x}
$$

Based on this formula, when the moving electrode touches the dielectric layer, $x=-0.8$, the capacitance is $5000 \mathrm{C}_{0}$. The capacitance changes to $1.25 \mathrm{C}_{0}$ when the moving electrode is at the equilibrium position of $x=0$. If we define the dimensionless parameter as follow. The capacitance can be expressed as:

$$
C=C_{0} \frac{1}{D_{e}+x}, \text { with } D_{e}=\frac{g_{\text {air }}^{0}}{d_{0}}+\frac{g_{d}}{\varepsilon_{r} d_{0}}
$$

Where $\mathrm{g}^{0}$ air is the air gap at equilibrium. If there is no dielectric layer, we have $\mathrm{g}_{\mathrm{d}}$, and $\mathrm{g}^{0}{ }_{\text {air }}=\mathrm{d}_{0}$ resulting in $D e=1$. For the dielectric layer given above, we have $D e=0.8002$ Therefore, parameter $D e$ is adequate to represent the presence of the dielectric layer. Figure $2.5 \mathrm{~b}$ shows the changes of capacitance as the top electrode moves from touching the dielectric layer to the equilibrium position and further moves to $x=0.8$, or touching the dielectric layer on the top electrode in a symmetric double cavity design.

\subsubsection{Model of electrostatic force of capacitor with dielectric layer}

The presence of the dielectric layer greatly affects the electrostatic forces between the electrodes. The electrostatic force can be modified using equation (14) for capacitance. We thus get:

$$
F=\frac{V^{2}}{2} C_{0} \frac{1}{\left(D_{e}+x\right)^{2}}
$$

When the plate displacement is $x=-0.8$, the electrostatic force changes from: 


$$
F=\frac{V^{2}}{2} C_{0} \frac{1}{0.2^{2}}
$$

for non-dielectric layer to:

$$
F=\frac{V^{2}}{2} C_{0} \frac{1}{0.0002^{2}}
$$

which represents one million fold increases. 


\section{CHAPTER 3}

\section{MODELING AND DYNAMIC STUDIES}

This chapter describes the modeling of the electrostatic power harvester with two capacitive cavities using finite element analysis. The modeling and simulation includes determining the resonance frequency and structural uniformity of the movable plate during vibration at various excitation amplitudes. In addition, we developed nonlinear dynamic models using Matlab to study the performance of the parallel plate variable capacitor with single capacitive cavity and two capacitive cavities. The model also includes the analysis of rocking instability (cause movable plates not in parallel to fixed plates) which may result in the reduction of the energy harvesting.

\subsection{Finite element modeling}

\subsubsection{Resonance frequency}

A significant fraction of vibration energy is distributed within lower range of the frequency spectrum, typically below $500 \mathrm{~Hz}$ [96]. Therefore, it is favorable to design vibrational energy harvester with a lower resonance frequency. To determine the resonance frequency of our power harvesting devices, the structure of the movable plate was modeled using COMSOL Multi-Physics finite element tool. As an example, Figure 3.1(a) shows the simulation of a movable plate with a thickness of $30 \mu \mathrm{m}$ and a serpentine shaped suspension beams with a width, thickness and total length (four serpentine turns) of $15 \mu \mathrm{m}, 5 \mu \mathrm{m}$ and $1485 \mu \mathrm{m}$, respectively. The simulation results clearly show that the plate vibrates uniformly without any distortion or warping. The 
model also shows that the suspended plate vibrates with a resonance frequency of $487 \mathrm{~Hz}$. The simulation was repeated on another design in which the movable plate thickness was increased to $50 \mu \mathrm{m}$ while other parameters were kept the same. In this case, the corresponding resonance frequency lowered to $372 \mathrm{~Hz}$ due to the heavier proof mass (Figure 3.1(b)). This result demonstrate that the resonance frequency of the power harvesting device can be adjusted by modifying both the thickness of suspension beams and the weight of the proof mass. As an example, a resonance frequency of $205 \mathrm{~Hz}$ was achieved when the thickness of the suspension beams and the thickness of the movable plate were reduced to $3 \mu \mathrm{m}$ and increased to $40 \mu \mathrm{m}$, respectively, as shown in Figure 3.1(c).

During the phase, we performed extensive FEM modeling for the movable plate with various dimensions and stiffness of suspension beam in order to accurately determine the resonance frequency. The reported resonance frequencies of several designs are listed in table 4 for comparison. It is noted that the above simulations were established without considering the impact of stoppers, and the parameters for the structure material are in ideal conditions. The experimental characterizations were performed on the device fabricated with dimensions of the movable plate and serpentine suspension beams the same as the models in Figure 3.1(a) and 3.1(c).

3.1.2 Nonlinear effects of squeeze film air damping, electrostatic force and elastic force of suspension

In an oscillating system, squeeze film air damping can significantly impact the excitation amplitude and resonance frequency of the oscillating plate, especially the MEMS oscillators with parallel plate structure. The stiffening effect of squeeze film air 
(a)

eigfreq_smsld(1)=487.103412 Subdomain: Total displacement [m] Deformation : Displacement

(b)
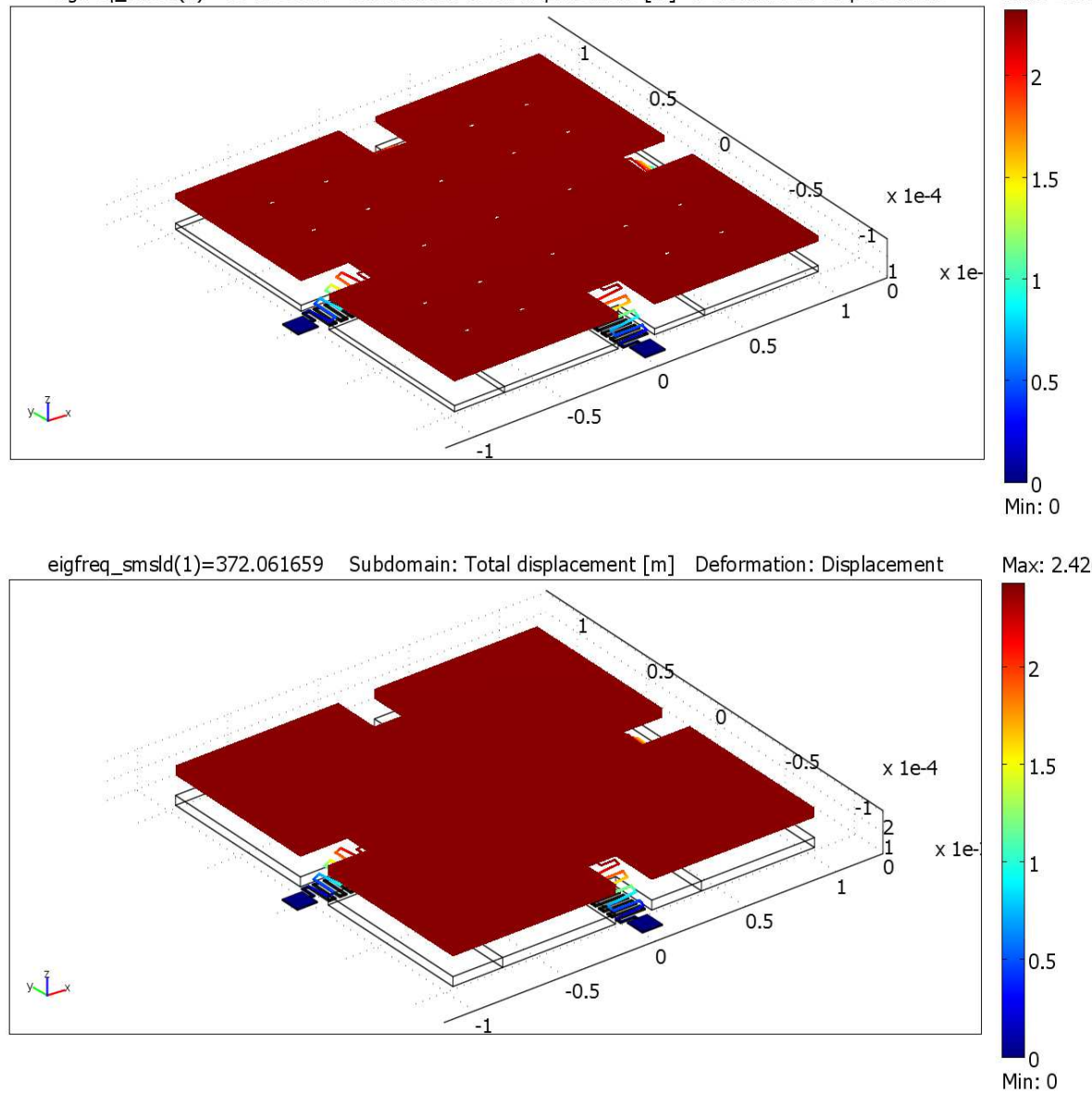

(c)

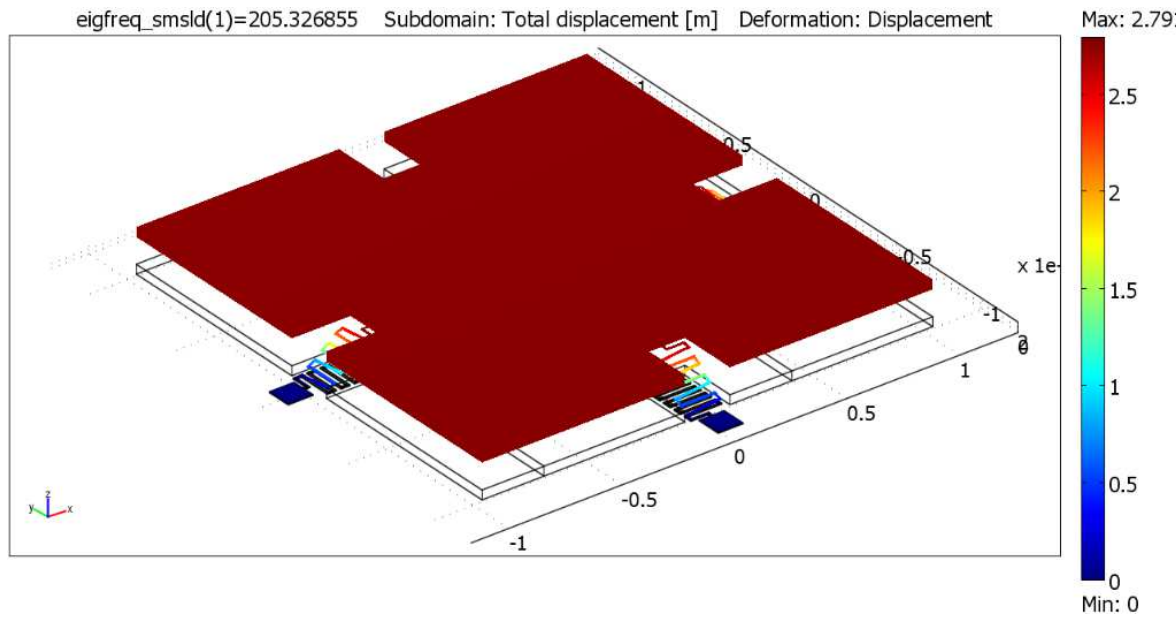

Figure 3.1. Finite Element Modeling of the suspended movable plate under fixed excitation amplitude. The plate and suspension beam thickness, and the determined resonance frequency were: (a) $30 \mu \mathrm{m}, 5 \mu \mathrm{m}$, and $487 \mathrm{~Hz}$, (b) $50 \mu \mathrm{m}, 5 \mu \mathrm{m}$, and $372 \mathrm{~Hz}$, and (c) $40 \mu \mathrm{m}, 3 \mu \mathrm{m}$, and $205 \mathrm{~Hz}$. 
Table 4. The expected resonant frequencies of the power harvesting devices with various dimensions determined using FEM Modeling.

\begin{tabular}{|c|c|c|c|c|c|}
\hline $\begin{array}{r}\text { Dimensions of } \\
\text { Movable } \\
\text { Plate } \\
\text { Thickness }(\boldsymbol{\mu m})\end{array}$ & Turns & $\begin{array}{c}\text { Length } \\
(\boldsymbol{\mu m})\end{array}$ & $\begin{array}{c}\text { Width } \\
(\boldsymbol{\mu m})\end{array}$ & $\begin{array}{c}\text { Thickness } \\
(\boldsymbol{\mu m})\end{array}$ & $\begin{array}{c}\text { Resonance } \\
\text { Frequency } \\
(\mathbf{H z})\end{array}$ \\
\hline 30 & 2 & 670 & 20 & 5 & 1280 \\
\hline 50 & 2 & 670 & 20 & 5 & 970 \\
\hline 30 & 3 & 1040 & 15 & 5 & 875 \\
\hline 50 & 3 & 1040 & 15 & 5 & 678 \\
\hline 30 & 5 & 1485 & 10 & 5 & 487 \\
\hline 40 & 5 & 1485 & 10 & 3 & 205 \\
\hline 50 & 5 & 1485 & 10 & 5 & 372 \\
\hline 30 & 7 & 2080 & 10 & 5 & 444 \\
\hline 50 & 7 & 2080 & 10 & 5 & 331 \\
\hline
\end{tabular}

damping can increase the resonance frequency of the movable plate. We currently do not have a numerical program to evaluate the nonlinear effect of squeeze film air damping mathematically due to the complicated nonlinear dynamic behaviors of the perforated movable plate. Analytical model of squeeze film air damping for such MEMS device requires modified Reynolds equation involving the damping effect of gas flow through holes on the movable plate. On the other hand, nonlinear effects of electrostatic force and elastic force of suspension can be optimized to broaden the bandwidth of the nonlinear oscillator. When the movable plate is getting close to the fixed plates, the electrostatic force between two plates is increasing nonlinearly. Therefore, the electrostatic force is softening in nature because it reduces the resonance frequency determined by the elastic suspension. The softening can be so strong that the elastic equilibrium may become 
unstable. We can optimize the softening effect by finding the appropriate DC bias voltages. We have investigated the nonlinear effects of squeeze film air damping, electrostatic force and elastic force of suspension experimentally. The results will be reported and discussed in chapter 5.

\subsection{Nonlinear dynamic simulation}

The advantages of the use of two capacitive cavities in our device was demonstrated by nonlinear dynamic simulations in Matlab developed by Dr. Zaichun Feng and Jianxiong Zhu $[97,102,103]$. The models prove that the two-cavity capacitor can achieve higher average power than conventional single cavity devices under a wide range of external forcing frequency and amplitude [97].

Designs of many micro devices take advantage of the symmetry for better performance, immunity to noise, and simpler analysis. When a symmetric structure is subjected to symmetric forcing, the symmetric response can become unstable leading to asymmetric responses. The occurrence of symmetry breaking bifurcation leads to complicated dynamic responses which often result in less desirable performances. There have been extensive dynamic studies on electrostatic MEMS power harvester using parallel plate structure which incorporates the nonlinear electrostatic, elastic, and squeeze film air damping in the model [98-100]. Most of researchers did not involve consideration of rotational motion of the movable plate which was normally treated as having only translational degree of freedom [101]. In Dr. Zaichun Feng and Jianxiong Zhu's work, they conduct numerical simulations to demonstrate the performances device with and without asymmetric dynamic responses resulting from the symmetry breaking [102, 103]. 
3.2.1 Dynamic simulation of MEMS variable capacitor with single cavity and two-cavity [97]

The first dynamic model was developed based on the electrostatic power harvester design with a single cavity. The movable plate is supported by four serpentine springs. Four stoppers are placed on fixed plate (Figure 3.2) to avoid short circuit during the collision of the movable plate with the fixed one. The schematic of the electrical control is shown in Figure 3.2. The output battery voltage is higher than that of the input power source. The charge across the plates is controlled by diodes. The whole arrangement was placed on a base to which vibration force was applied.

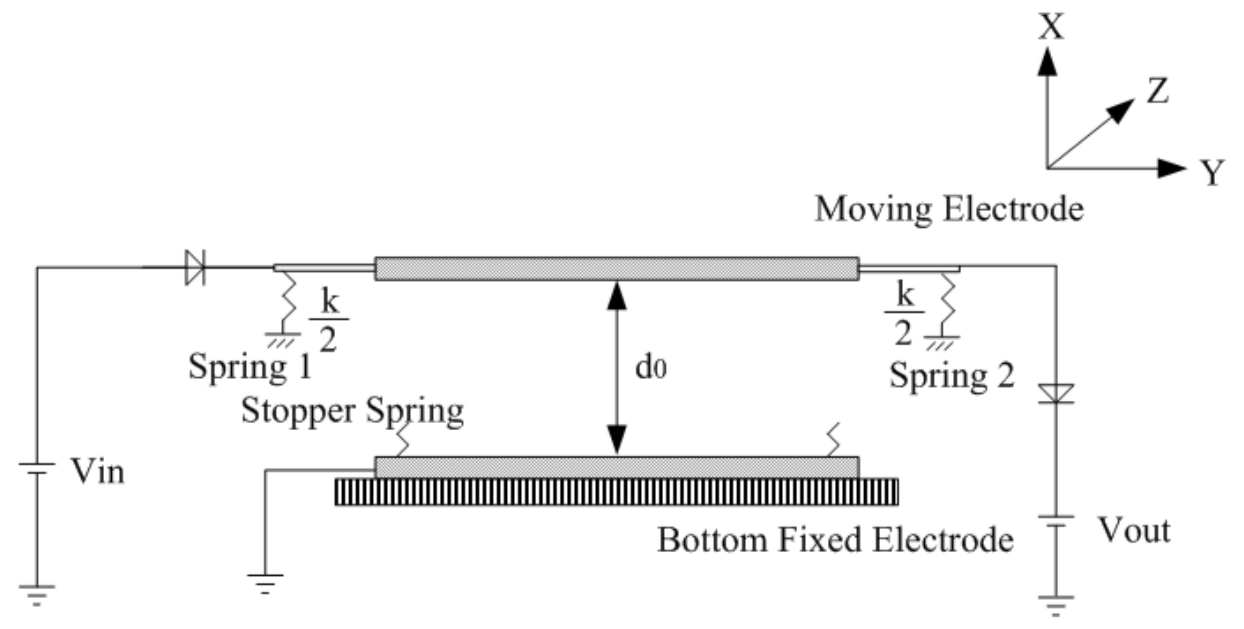

Figure 3.2. A modeling schematic of conventional single cavity energy harvester showing the arrangement of springs and electrical control circuit [97].

The model of the MEMS capacitive plates with two cavities was similarly established which consists of two variable capacitors with gap 1 and gap 2. The input and output DC voltage batteries are connected to fixed plates' electrodes and movable plate electrode is made ground (see Figure 3.3). Charge across the plates is controlled by diodes. 
In two cavity model, the stopper springs are placed on both the top and bottom fixed plates. Therefore, as voltage across the suspended plate reaches to the pull down voltage, the plate starts striking on the stoppers (springs). At low forcing frequency and amplitude of vibration, the plate will stick either at upper or at bottom of the fixed plate stopper because of the pull-down voltage. The specifications used in the simulation are shown in Table 5.

The average power for single cavity and two cavity models are computed using

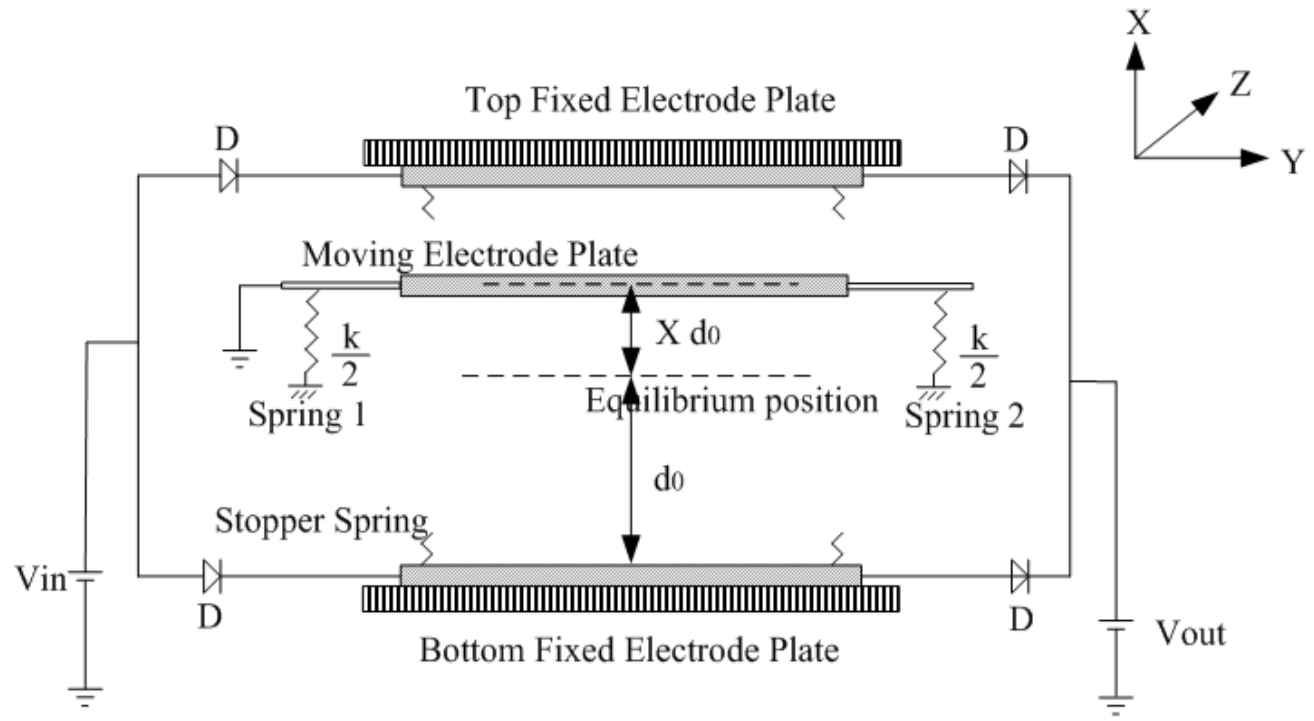

Figure 3.3. A modeling schematic of two-cavity energy harvester showing the arrangement of springs and electrical control circuit [97].

Table 5 Specifications that are used in the Matlab simulation for single cavity and two cavity models. The two cavity model has a $30 \mu \mathrm{m}$ top air gap. The electroplated Nickel density is $8912 \mathrm{~kg} / \mathrm{m}^{3}$.

\begin{tabular}{|c|c|c|c|c|c|c|}
\hline $\begin{array}{c}\text { Plate } \\
\text { area } \\
\left(\mathbf{m m}^{2}\right)\end{array}$ & $\begin{array}{c}\text { Plate } \\
\text { thickness } \\
(\boldsymbol{\mu m})\end{array}$ & $\begin{array}{c}\text { Air } \\
\text { Gap } \\
(\boldsymbol{\mu m})\end{array}$ & $\begin{array}{c}\text { Resonance } \\
\text { frequency } \\
(\mathbf{H z})\end{array}$ & $\begin{array}{c}\text { Pull- } \\
\text { down } \\
\text { voltage } \\
(\mathbf{V})\end{array}$ & $\begin{array}{c}\text { Input } \\
\text { voltage } \\
(\mathbf{V})\end{array}$ & $\begin{array}{c}\text { Downstream } \\
\text { voltage }(\mathbf{V})\end{array}$ \\
\hline $2 \times 2$ & 30 & 30 & 800 & 12 & 5 & 15 \\
\hline
\end{tabular}


Table 6. Comparison of simulation results of average power (Average Current $\times$ Output Voltage) produced by a single cavity model and a two-cavity model [97].

\begin{tabular}{|c|c|c|c|c|c|c|}
\hline \multirow{2}{*}{$\begin{array}{c}\text { Forcing Amplitude } \\
(\boldsymbol{\mu m})\end{array}$} & \multicolumn{6}{|c|}{$\begin{array}{c}\text { Average Power }(\boldsymbol{\mu W}) \text { Produced By One Cavity Model } \\
\text { Under Different Forcing Frequency and Amplitude }\end{array}$} \\
\cline { 2 - 7 } & $\mathbf{0 . 4 k H z}$ & $\mathbf{0 . 8 k H z}$ & $\mathbf{1 . 2 k H z}$ & $\mathbf{1 . 6 k H z}$ & $\mathbf{2 k H z}$ & $\mathbf{2 . 4 k H z}$ \\
\hline 100 & 6.802 & 17.02 & 24.06 & 13.10 & 27.00 & 35.06 \\
\hline 80 & 5.586 & 11.64 & 23.79 & 11.95 & 33.51 & 22.43 \\
\hline 60 & 4.743 & 15.27 & 21.75 & 16.89 & 28.73 & 25.75 \\
\hline 40 & 0.104 & 13.56 & 21.92 & 31.54 & 29.37 & 22.21 \\
\hline 20 & 0 & 10.28 & 20.68 & 27.89 & 19.08 & 21.40 \\
\hline \multirow{2}{*}{$\begin{array}{c}\text { Forcing Amplitude } \\
(\boldsymbol{\mu m})\end{array}$} & $\mathbf{A v e r a g e}$ Power $(\boldsymbol{\mu W})$ Produced By Two Cavity Model \\
\cline { 2 - 7 } & $\mathbf{U n d e r}$ & $\mathbf{D i f f e r e n t}$ Forcing Frequency and Amplitude \\
\hline 100 & $\mathbf{0 . 4 k H z}$ & $\mathbf{0 . 8 k H z}$ & $\mathbf{1 . 2 k H z}$ & $\mathbf{1 . 6 k H z}$ & $\mathbf{2 k H z}$ & $\mathbf{2 . 4 k H z}$ \\
\hline 80 & 10.10 & 26.19 & 42.23 & 61.77 & 78.90 & 88.21 \\
\hline 60 & 9.519 & 24.58 & 38.09 & 52.02 & 56.91 & 80.38 \\
\hline 40 & 9.815 & 22.29 & 35.59 & 43.22 & 59.61 & 70.65 \\
\hline 20 & 0 & 19.92 & 33.88 & 46.12 & 58.76 & 70.31 \\
\hline & 0 & 20.42 & 30.18 & 41.49 & 53.90 & 62.76 \\
\hline
\end{tabular}

Matlab programs for simulation (see Table 6). We obtained a maximum $35.1 \mu \mathrm{W}$ of power out of single cavity model and a maximum $88.2 \mu \mathrm{W}$ of power out of two-cavity model in the simulation, which concludes that two-cavity model generate more power compared to a single cavity model. In addition, as we increase the amplitude or forcing frequency, the average power increases. It is noted that some exceptions are observed.

3.2.2 Dynamic simulation of MEMS variable capacitor with two-cavity involving rocking instability $[102,103]$

In the real world, rocking instability is inevitable for parallel movable plates 
structure (perturbations that may result in plates being no longer in parallel to each other). In other words, the movable plate behaves both with vertical and rotational motion so that the tips of the movable plate are very likely to hit the stoppers or even the fixed plates. A schematic of the typical model in Figure 3.4 illustrates the device with onset of rocking instability. When the device is being charged, the voltage is varying as the movable plate

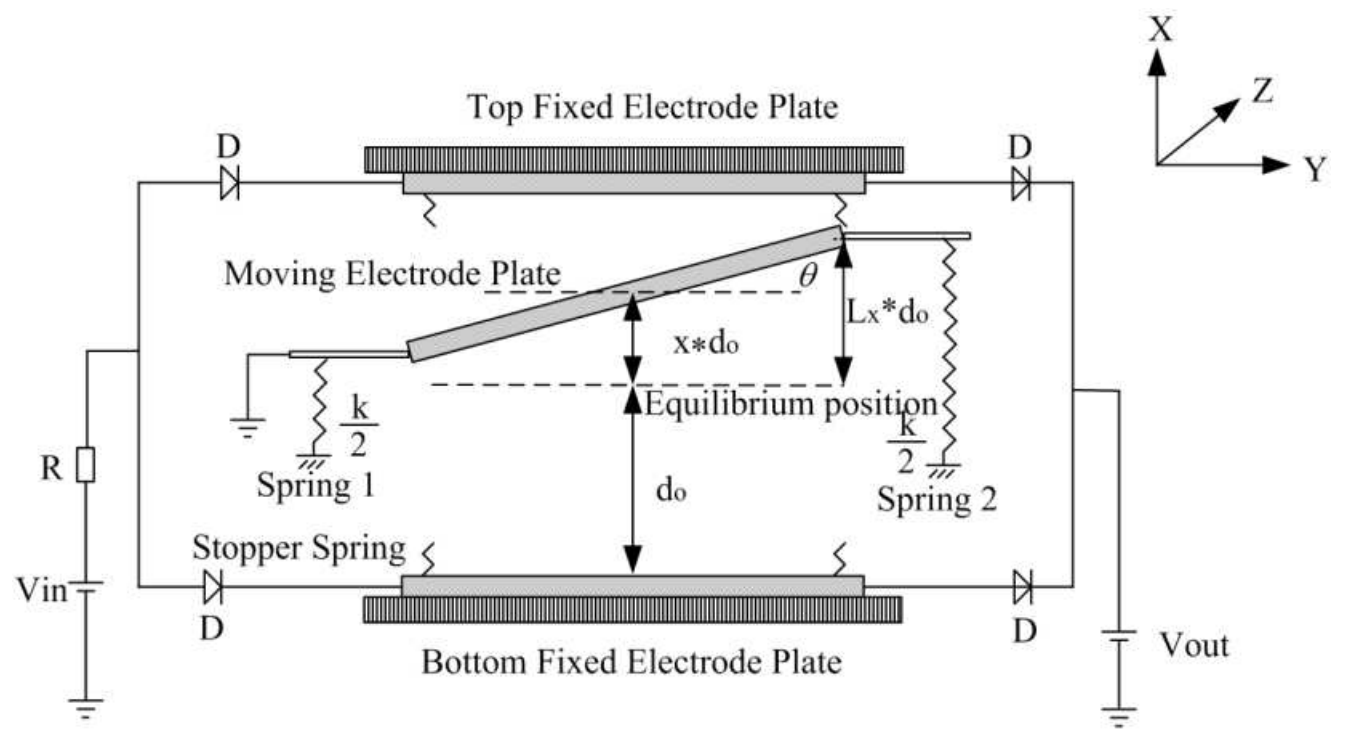

Figure 3.4. A modeling schematic of electrostatic MEMS energy harvester with dualcavity involving rocking instability [103].

is driven into motion by sinusoidal excitations. Low DC voltage battery on the left of Fig. 4 is used to charge the device, and the raised voltage as the plate vibrates is released to the battery on the right. The value of $V_{\text {in }}$ and $V_{\text {out }}$ should be determined by the specific application. They were chosen based upon general application for illustrative purpose in this modeling, $\mathrm{V}_{\text {in }}=5 \mathrm{~V}$ and $\mathrm{V}_{\text {out }}=15 \mathrm{~V}$. Diodes are used to prevent the charge from flowing backwards. It should be pointed out that the diodes in this dynamic model are assumed to be ideal (leakage and parasitic capacitance are ignored). The $\mathrm{R}$ is a load resistor which is used to prevent the current from burning out the diodes. 
Table 7. Comparison of simulation results of average power (Average Current $\times$ Output

Voltage) produced by a two-cavity model without and with rocking instability [103].

\begin{tabular}{|c|c|c|c|c|c|c|}
\hline \multirow{3}{*}{$\begin{array}{l}\text { Excitation } \\
\text { Amplitude } \\
\underset{(\mu \mathrm{m})}{ }\end{array}$} & \multicolumn{6}{|c|}{$\begin{array}{c}\text { Average Power }(\mu W) \text { Produced By a Dual-Cavity Model Without } \\
\text { Rocking Instability }\end{array}$} \\
\hline & \multicolumn{6}{|c|}{ Excitation Frequency $=\gamma \times$ Natural Frequency } \\
\hline & $\gamma=0.5$ & $\gamma=1.0$ & $\gamma=1.5$ & $\gamma=\mathbf{2 . 0}$ & $\gamma=2.5$ & $\gamma=3.0$ \\
\hline 80 & 10.54 & 27.85 & 49.74 & 68.68 & 93.66 & 121.9 \\
\hline 60 & 10.35 & 23.92 & 37.94 & 58.86 & 74.71 & 94.74 \\
\hline 40 & 0 & 21.75 & 42.64 & 58.52 & 64.7 & 93.39 \\
\hline 20 & 0 & 21.02 & 32.98 & 46.03 & 59.97 & 76.15 \\
\hline \multirow{3}{*}{$\begin{array}{c}\text { Excitation } \\
\text { Amplitude } \\
(\mu \mathrm{m})\end{array}$} & \multicolumn{6}{|c|}{$\begin{array}{c}\text { Average Power }(\mu W) \text { Produced By a Dual-Cavity Model With } \\
\text { Rocking Instability }\end{array}$} \\
\hline & \multicolumn{6}{|c|}{ Excitation Frequency $=\gamma \times$ Natural Frequency } \\
\hline & $\gamma=0.5$ & $\gamma=1.0$ & $\gamma=1.5$ & $\gamma=2.0$ & $\gamma=2.5$ & $\gamma=3.0$ \\
\hline 80 & 4.29 & 1.34 & 9.81 & 23.52 & 27.84 & 30.24 \\
\hline 60 & 5.42 & 9.84 & 13.62 & 21.36 & 29.24 & 39.63 \\
\hline 40 & 0 & 9.82 & 10 & 18.70 & 24.7 & 43.14 \\
\hline 20 & 0 & 17.25 & 6.31 & 0.17 & 12.14 & 31.09 \\
\hline
\end{tabular}

The results have shown that when the motion of the movable plate is dominated by the separate impacts of two tips, the capacitance change of top and bottom cavity is much less than when the plates are in parallel. The harvested power is thus much reduced. The harvested power from the same model with and without the rocking instability over a broad range of frequencies and forcing amplitude are shown in Table 7. It should be pointed out that the rocking instability is initiated by involving a very small deflection angle on the movable plate in the initial condition, and the movable plate makes contact with the end stops in the simulations for most of these forcing parameters. We can see 
that the power generation is dramatically lowered by the onset of rocking instability in all conditions. However, it is noted that the harvested power in the dynamic studies is overestimated otherwise. 


\section{CHAPTER 4}

\section{FABRICATION}

This chapter first introduces the micromachining technologies used in the fabrication of the MEMS variable capacitor with two capacitive cavities for power harvesting. This includes photolithography, wet etching, reactive ion etching, Radio Frequency (RF) sputter deposition of thin films, electroplating, and lift-off process. The fabrication processes will be described in details step by step.

\subsection{Microfabrication techniques and materials}

\section{A. Photolithography}

Photolithography is an optical method for transferring a geometric pattern from a photo mask to a substrate coated with a light-sensitive chemical known as photoresist based on radiation-induced structural degradation. Photoresist is a photosensitive organic material which is generally composed of three major components: polymer, photo active compound, and solvent. A positive or negative image can be produced on the substrate depending on the chemical nature of photoresist. Photolithography process has been widely used in microfabrication to selectively remove parts of a thin film or bulks of a substrate. It has become a basic technique used to define and transfer patterns in the most of micromachining and integrated circuit fabrication processes.

Prior to any micromachining processes, the substrates such as silicon wafers or glass slides, must be cleaned thoroughly using standard cleaning processes. This include solvents (acetone, methanol, and deionized water) for cleaning dust and organic residue, 
piranha solution, a mixture of sulfuric acid and hydrogen peroxide, for deep cleaning the organic contaminations, such as photoresist and oil residues, and buffered hydrofluoric acid (HF) for removing the naturally formed thin oxide film. After cleaning of substrate, dehydration baking is sometimes necessary to eliminate any moisture adsorbed by substrate surface. The substrate is then placed on a spinner and held by vacuum. This is followed by dispensing photoresist onto the substrate and spinning at a high speed, e.g., 3000 RPM, to coat the substrate with photoresist with a uniform thickness. During the spinning process, a large proportion of solvent in photoresist is evaporated. The thickness of the photoresist layer is given by [104]:

$$
t=\frac{k p^{2}}{\sqrt{w}}
$$

where $\mathrm{k}$ is the spinner constant, $\mathrm{p}$ is the photoresist solid contents in percent which indicates the viscosity, and $\mathrm{w}$ is the rotational speed in thousand RPM. The equation clearly indicates that the coated photoresist thickness is independent of the dispensing volume of photoresist and its acceleration rate, but it is dependent of the spinning speed and time.

Although the solvent concentration of photoresist are much reduced from its initial level right after spin coating, it is still too high to create a sufficient difference in developing rates between the exposed and unexposed portions. Therefore, a process called soft baking is used to further evaporate the solvent in photoresist, consequently, densifying the photoresist and improving the adhesion to the substrate. Soft baking can be performed by convection oven or hotplate. After the soft baking, the substrate is exposed with ultraviolet light (UV) through a photomask that selectively exposes the photoresist 
using a high precision mask aligner as shown in Figure 4.1(b). In this project, the photolithography process was performed using Karl-Suss MA6-BA6 Mask Aligner (Figure 4.1(a)) with a resolution up to $1 \mu \mathrm{m}$. The photomask contains chromium patterns on soda lime glass or quartz glass.

(a)

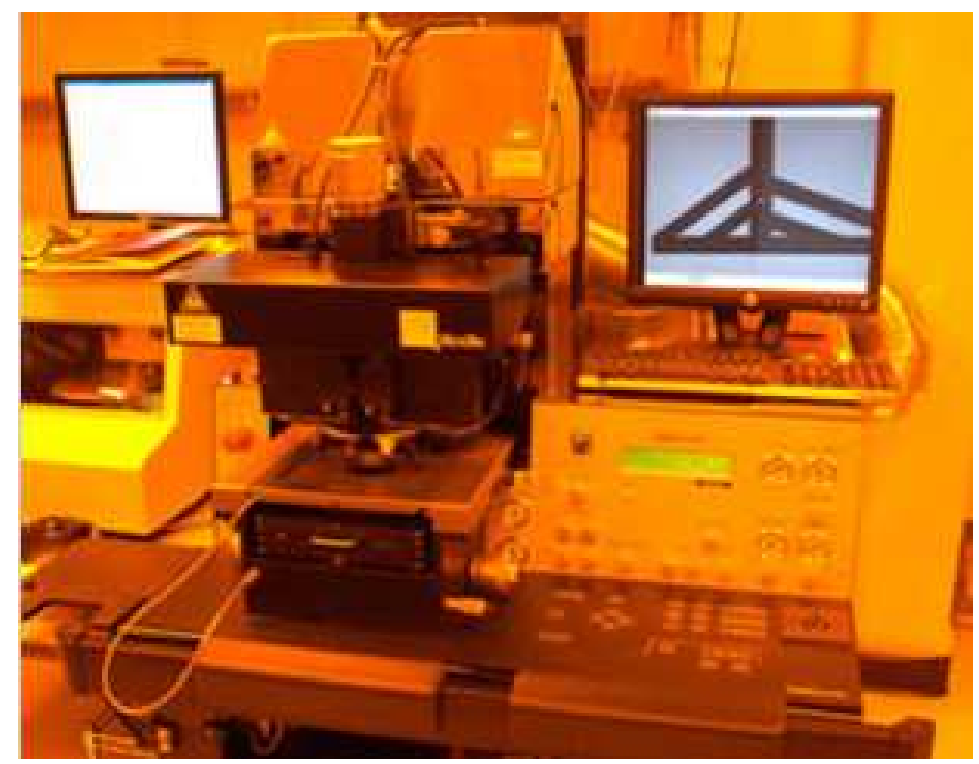

(b)

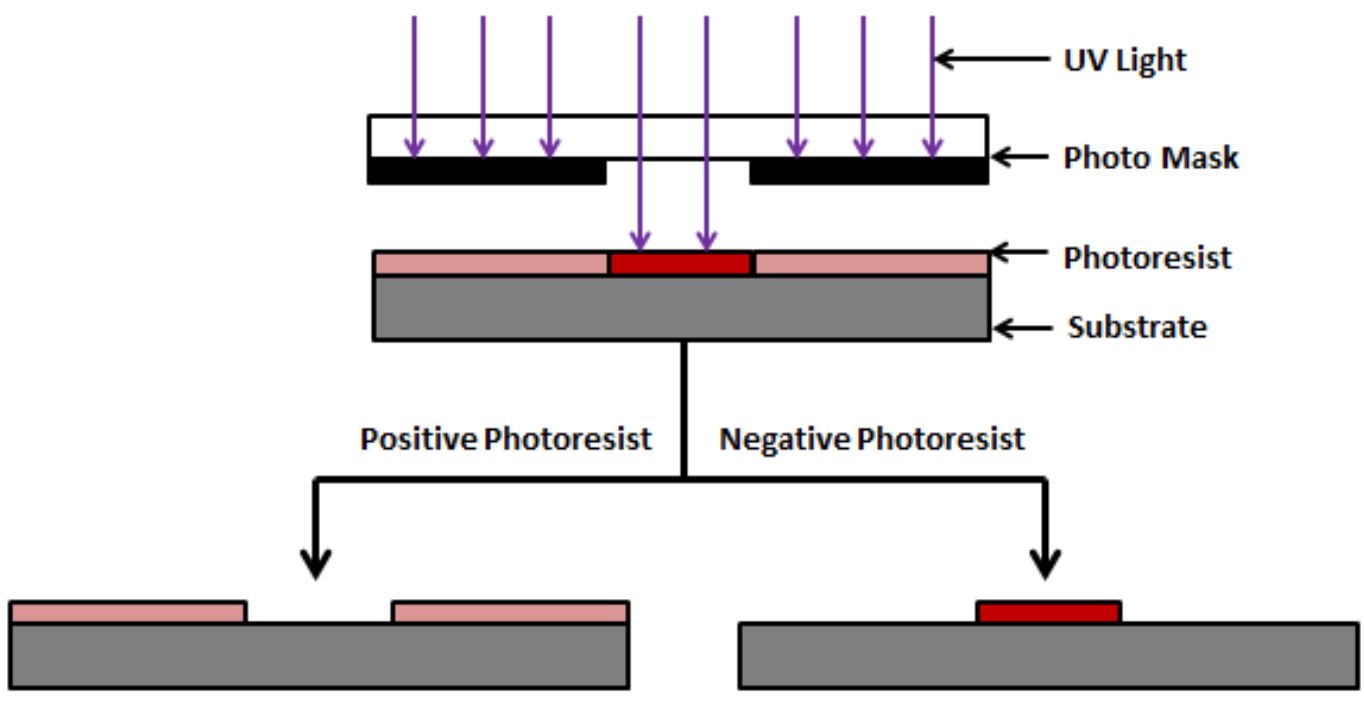

Formed Pattern After Development

Figure 4.1. (a) Karl-Suss MA6-BA6 Mask Aligner in the Microsystem Research Lab of the University of Missouri, (b) a schematic of the photolithography process using positive and negative photoresist. 
The wavelength and energy of UV light must be selected according the properties and thickness of the coated photoresist layer. Because the diffraction is an inevitable consequence of the wave nature of light, the resolution of any photolithographic system is limited both by configuration and the wavelength of light used:

$$
R=k \frac{\lambda}{N A}
$$

Where $R$ is the resolution (also known as minimum feature size or critical dimension), $\lambda$ is the wavelength of light, $k$ and $N A$ is configuration constant on the order of 1 and 1.6 respectively. The exposed area is either degraded for a positive photoresist or polymerized for a negative photoresist due to the UV induced chemical reaction.

In addition, there is an optional step known as post exposure baking (PEB) for certain types of photoresist after the UV exposure but prior to the development. PEB is necessary in some cases to improve critical dimension control, and to enhance photoresist profile [105]. Particularly, for chemically amplified photoresists, such as SU-8 and AZ40XT, PEB is required and critical for the chemical reaction using the photo generated acid as a catalyst. The substrate with the UV exposed photoresist will be developed in a special solution known as a developer. The exposed area would be dissolved and the unexposed area would stay in the case of positive photoresist. The same logic in reverse, the exposed area in a negative photoresist would stay and unexposed area will be dissolved.

Finally, it is sometimes necessary to hard bake the patterned photoresist at higher baking temperature than that of soft baking temperature. The hard baking is used for hardening the photoresist so that it is able to undergo the micromachining processes such as wet etching or electroplating processes. It is also used to cause photoresist to reflow. 
The patterned photoresist layer can then be used as a mask for wet or dry etching, lift off process of thin films, or as a sacrificial layer.

B. Wet chemical etching

Wet chemical etching is a process that utilizes liquid chemicals or etchants including acids, bases or other chemicals to dissolve away unwanted materials from the substrate or thin films, forming specific patterns defined by photoresist masking layer or other material masks on the substrate. Materials not covered by photoresist masks are etched away by the chemicals while those covered by the masks are left intact.

Wet chemical etching can be classified as isotropic and anisotropic as shown in Figure 4.2. In the former, etching rate is the same in both horizontal and vertical directions (Figure 4.2(a)), while in the later, etching rate is different in the horizontal and vertical directions. Figure 4.2(b) shows an etching process that proceeds in only vertical direction which is also known as completely anisotropic.

(a)

Isotropic Etching

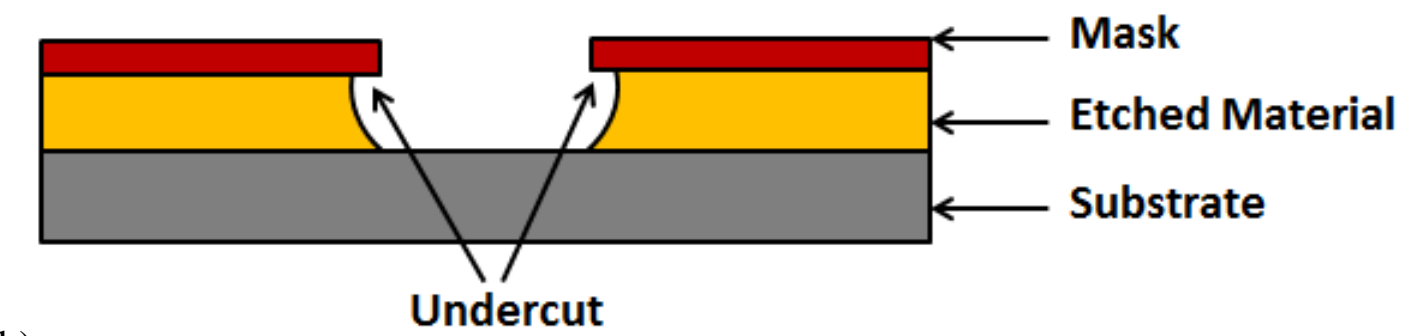

(b)

Anisotropic Etching

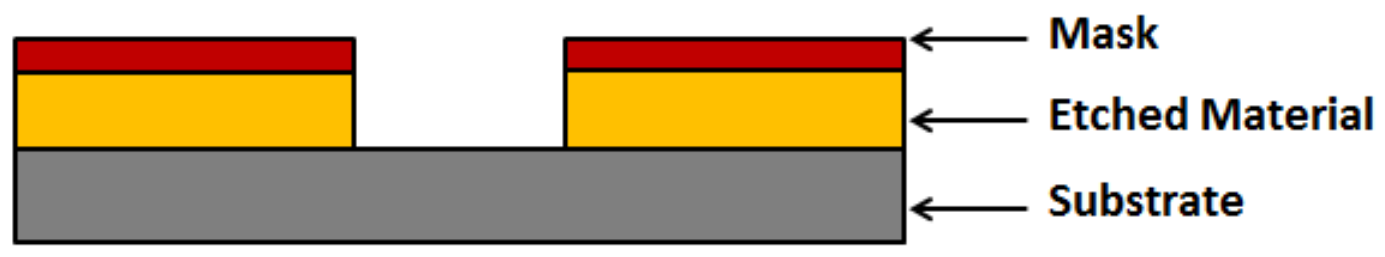

Figure 4.2. The isotropic and anisotropic etching profile. 
The isotropic and anisotropic etching can be classified using lateral etch ratio:

$$
R_{L}=\frac{r_{H}}{r_{V}}
$$

Where $r_{H}$ is horizontal etch rate, $r_{H}$ is vertical etch rate. When $R_{L}=1$, the etching is isotropic, $0<R_{L}<1$, the etching is anisotropic, and $R_{L}=0$, the etching is completely anisotropic. Wet chemical etching is generally isotropic even though a mask is present since the liquid etchant can penetrate underneath the mask. Isotropic etching creates round corners and undercuts the mask because it etches in all directions at the same etch rate, as shown in Figure 4.2(a). In our fabrication process, only isotropic wet chemical etching were used.

C. Dry Etching

Dry etching is one of the most widely used micromachining processes in semiconductor and MEMS manufacturing. Dry etching uses plasma generated free radicals to remove materials only from the area dictated by a photoresist pattern created using the photolithography. The Reactive Ion Etch (RIE) tool is capable of micromachining holes or other patterns with vertical side walls.

The dry etching is also known as Reactive Ion Etch (RIE) which is a plasma process where radio frequency (RF) discharged or excited species (radicals, ions) are used to etch substrate or thin films in a low pressure chamber. RIE is a synergistic process between chemically reactive species and energetic ion bombardment, thus it is faster than either pure physical ion bombardment or spontaneous chemical etching. RIE has anisotropic character, because ion bombardment is directional, which has reduced lateral etch rate and produced vertical or nearly vertical sidewalls. RIE is essential when 
narrow lines or channels are needed, or when high aspect ratio structures need to be fabricated. RIE of silicon is independent of crystal planes, and therefore any shape can be fabricated, unlike anisotropic wet etching. The RIE can also be used to produce isotropic etch profiles by changing the etch process parameters.

We have used the Oxford Plasmalab 80 Reactive Ion Etcher in this project as shown in Figure 4.3(a). This equipment is dedicated for processes involved with fluorine based gas chemistries, such as $\mathrm{CHF}_{3}$ and $\mathrm{SF}_{6}$, for anistropically etching silicon, silicon nitride, and silicon dioxide. The general configuration of a capacitively coupled plasma RIE is shown in Figure 4.3(b). The plasma is energized by a radio frequency power supply operated at frequency of $13.56 \mathrm{MHz}$. A capacitor is connected to the bottom electrode to block DC current while the top electrode is grounded. Moderate vacuum (usually in order of $1 \times 10^{-5}$ torr) is needed to increase the mean free path of charges and, consequently, for sustaining the plasma in the chamber. In our fabrication process, RIE is used to etch silicon substrate with a gas mixture of sulfur hexafluoride and oxygen $\left(\mathrm{SF}_{6}+\right.$ $\mathrm{O}_{2}$ ). $\mathrm{SF}_{6}$ is the main reactive gas for silicon etching, and $\mathrm{O}_{2}$ is additive gas for uniformity improvement and control of polymer contamination. The reactive plasma for silicon etching is composed of electrons, ions, $\mathrm{SF}_{6} / \mathrm{O}_{2}$ molecules and $\mathrm{F} / \mathrm{O}$ radicals. F radicals are generated from collisions of energetic electrons and $\mathrm{SF}_{6}$ molecules, and this process is called dissociation which can be written as:

$$
S F_{6}+e^{-} \longrightarrow S F_{5}+F+e^{-}
$$

The $F$ radicals can chemically react with silicon generating volatile byproducts of $\mathrm{SiF}_{4}$ in the process:

$$
\mathrm{Si}+\mathrm{F} \longrightarrow \mathrm{SiF}_{4} \uparrow
$$


(a)

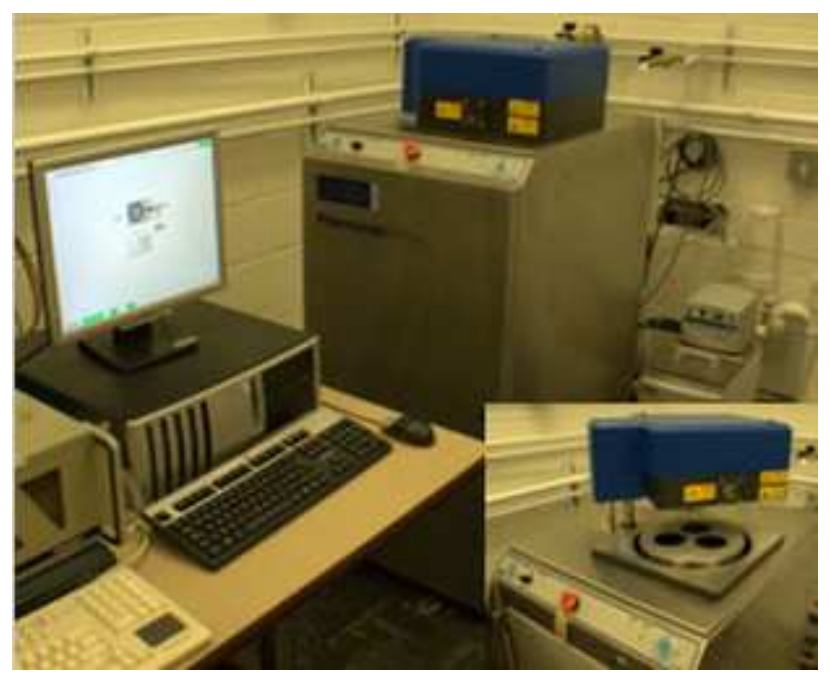

(b)

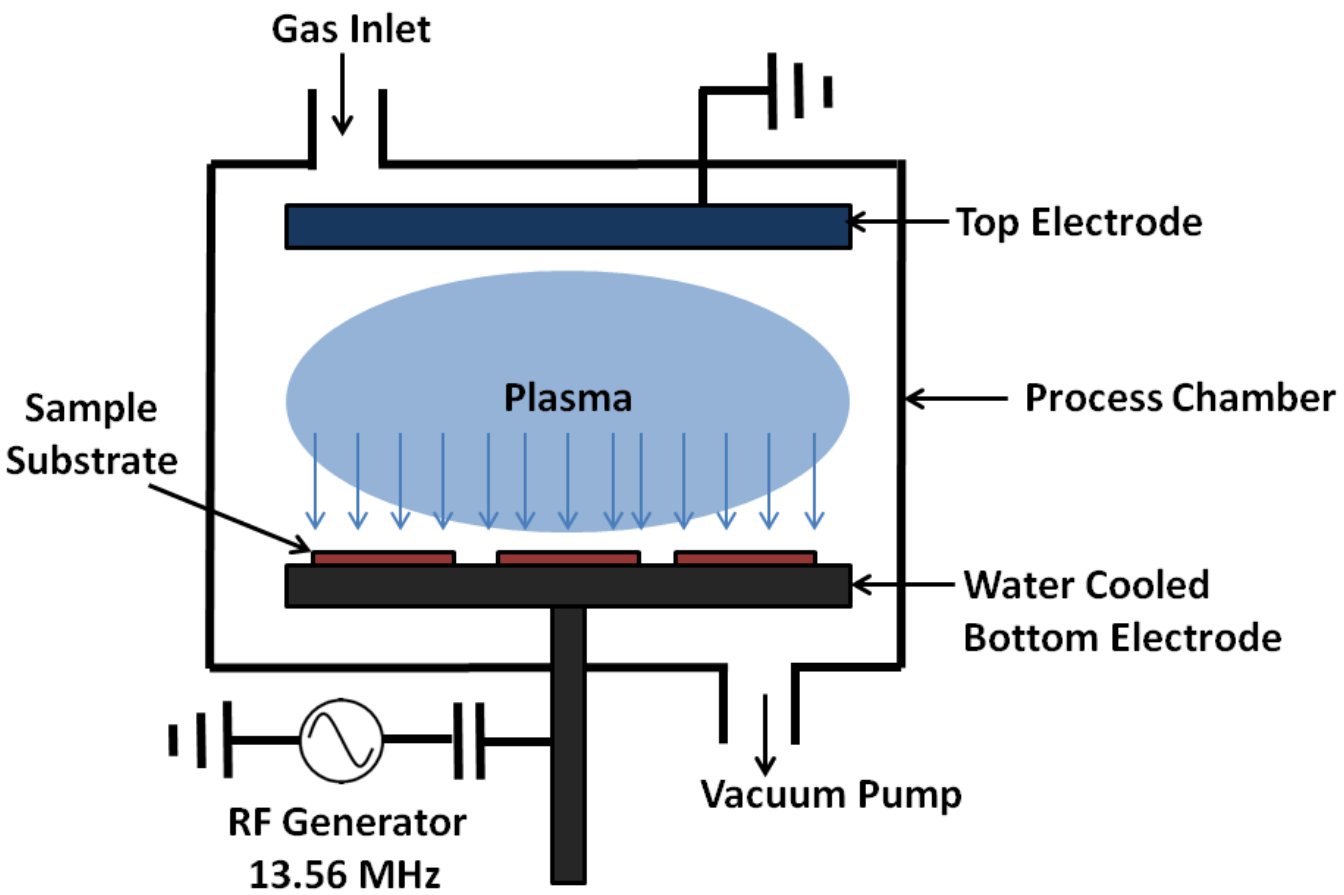

Figure 4.3. (a) The Oxford Plasmalab 80 Reactive Ion Etcher in the Microsystem Research Lab of University of Missouri, (b) a typical capacitively coupled plasma RIE configuration.

Similarly, O radicals are dissociated from collisions of energetic electrons and oxygen molecules as:

$$
\mathrm{O}_{2}+e^{-} \longrightarrow \mathrm{O}+\mathrm{O}+e^{-}
$$


$\mathrm{O}$ radicals are very chemically reactive with organic polymers at room temperature as:

$$
\mathrm{C}_{x} \mathrm{H}_{y} \mathrm{O}_{z}+\mathrm{O} \longrightarrow \mathrm{CO}_{x}+\mathrm{H}_{2} \mathrm{O}
$$

The byproducts $\left(\mathrm{SiF}_{4}, \mathrm{CO}_{\mathrm{x}}\right.$, and $\left.\mathrm{H}_{2} \mathrm{O}\right)$ of the ionic chemical reactions are volatile at low pressure and pumped out of the vacuum chamber.

D. Sputter deposition

Sputter deposition is a term refers to a physical vapor deposition (PVD) technique wherein atoms or molecules are sputtered from a target material by high energy particle bombardment so that the atoms or molecules can condense on a substrate as a thin film. Sputter deposition has become one of the most widely used techniques for depositing various metallic thin films including gold, chromium, aluminum, metallic alloys, platinum, etc., on silicon wafers or other substrates.

During the sputtering process, the target material is sputtered away by the bombardment of target with an inert ion (Argon ion in most cases). The ions are accelerated by negatively biased potential gradient and bombard the target material. Atoms or molecules are sputtered from the target to every direction and deposited onto a substrate and chamber walls. Almost any material can be sputtered off if sufficiently high energy plasma can be generated. Magnetron sputtering system (Figure 4.4(b)) is a modified version of traditional sputtering system. It utilizes a special configured magnetic field and an electric field to increase the plasma density of the area close to the target which allows a much higher deposition rate at the substrate. The Figure 4.4(a) shows the Kurt Lesker AXXIS Multi-Deposition System in the Microsystem Research Lab of University of Missouri, which possesses DC and RF powers designed for different 
sputtering processes. This system is dedicated in sputtering deposition of $\mathrm{Cr}, \mathrm{Au}, \mathrm{SiO}_{2}$ and $\mathrm{Si}_{3} \mathrm{~N}_{4}$ thin films in this project.

(a)

(b)
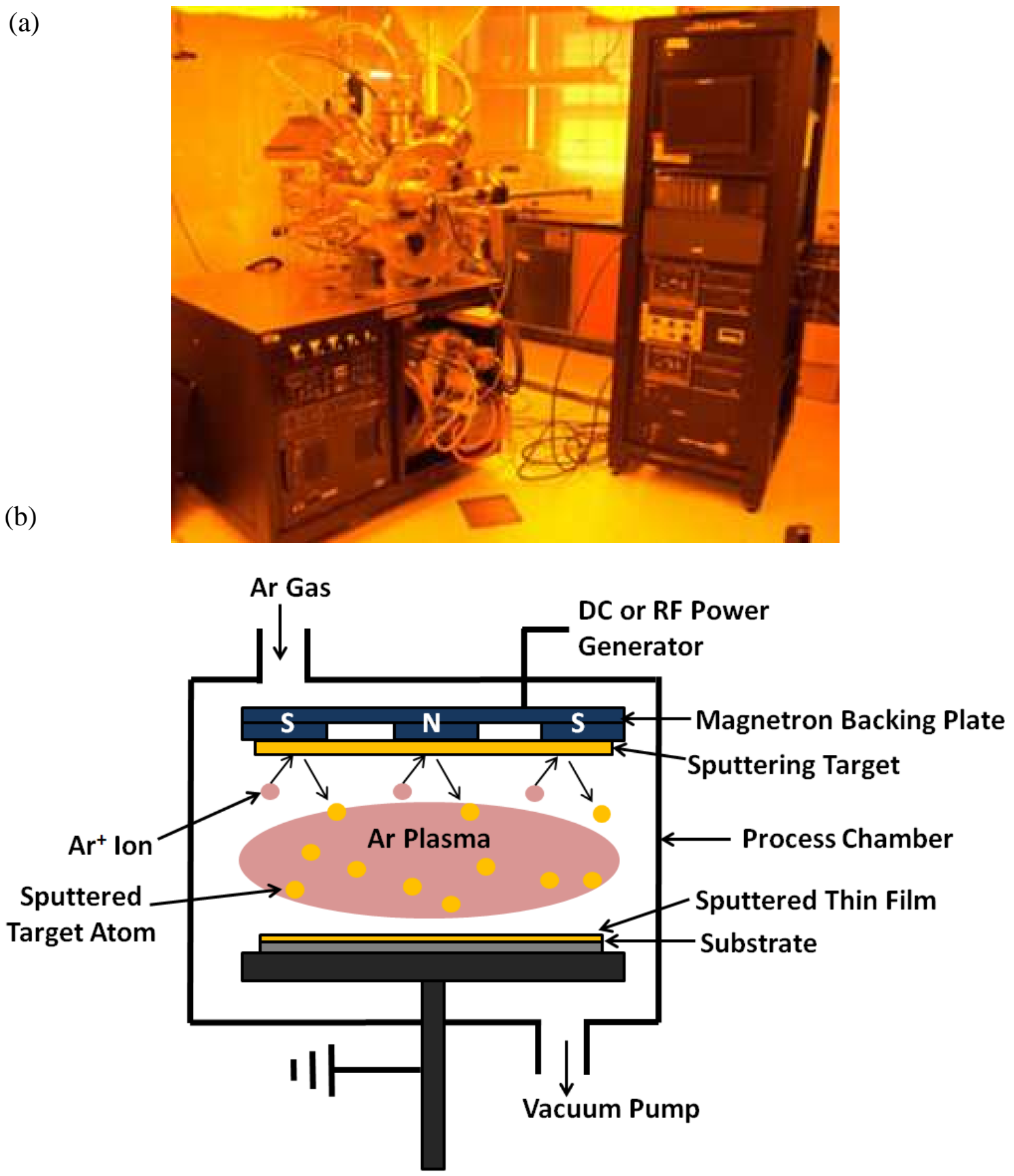

Figure 4.4. (a) A typical magnetron sputtering system configuration, (b) Kurt Lesker AXXIS Multi-Deposition System in the Microsystem Research Lab of University of Missouri. 
In RF magnetron sputtering process, magnets are placed behind the target as shown in Figure 4.5. The magnetic fields originate from the North Pole and end at the South Pole while the intensity of the magnetic field decreases as the distance from the magnets increases. Moving electrons in the plasma area close to the target are trapped by those magnetic fields and more collisions and ionizations take place in the region. Therefore, plasma density around target in magnetron sputtering process is much higher than in conventional sputtering process. Higher plasma density generates more argon ions which are swept to the target by the negative potential for the ion bombardment resulting in a higher deposition rate. Another advantage of magnetron sputtering is the capability of the deposition at a lower pressure, normally at 4 mTorr. Less collision between atoms sputtered from the target and argon atoms at low pressure would benefit the deposited film properties because sputtering is basically a type of physical vapor depositions. However, special care must be taken for magnetic material deposition, such as nickel and iron, when using magnetron sputtering, because magnetic field may be significantly decreased by the magnetic material of target.

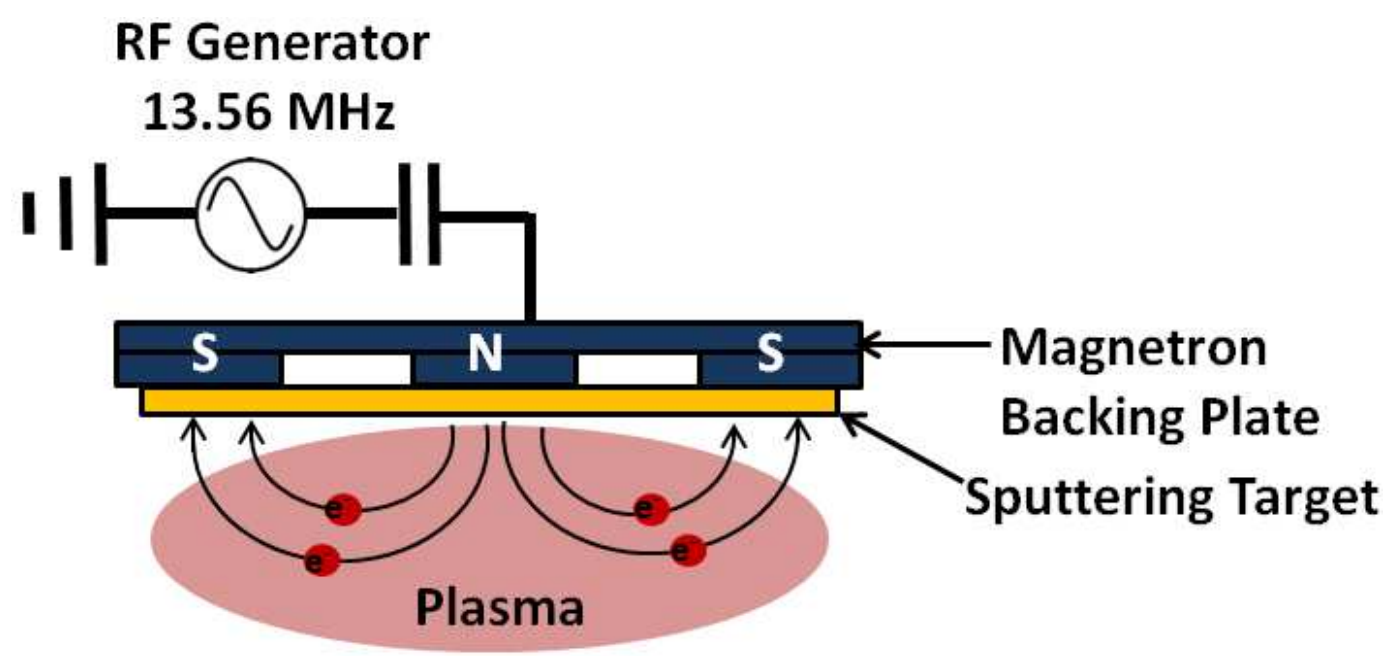

Figure 4.5. A schematic of the RF magnetron sputtering magnetic poles and field. 
The sputter deposition has some disadvantages, for examples, the rates of deposition of some materials, such as $\mathrm{SiO}_{2}$ and $\mathrm{Si}_{3} \mathrm{~N}_{4}$, are relatively low, and it has tendency to introduce impurities in the deposited films.

\section{E. Electroplating}

Electroplating is also called electrodeposition process which is used for producing a dense, uniform, and adherent coating, usually of metal or alloys, upon a surface by the act of electric current. Figure 4.6(a), 4.6(b) and 4.6(c) show a schematic of an electrolytic setup for electroplating and the real experimental setup used for $\mathrm{Ni}$ and $\mathrm{Au}$ electroplating in this project. The deposition of a metallic coating onto a sample is achieved by putting a negative charge on the object to be coated and immersing it into a solution which contains a metal salt to be deposited. The metallic ions of the salt carry a positive charge and are attracted to surface of the substrate to be electroplated. When they reach the negatively charged surface of the substrate, it provides electrons to reduce the positively charged ions to metallic form [106].

A standard electroplating process for MEMS fabrication can be described as follows: First, a thin film of metal seed layer, e.g., Cr and Au layers, is deposited onto the substrate. Second, a thick photoresist is patterned by photolithography to serve as a mold for electroplating. The deposition rate of electroplating can be controlled by the applied DC current density. In addition, some properties of electroplated layer such as surface smoothness can be tuned by utilizing pulsed electroplating technique or biased alternating current electroplating technique. Finally, the photoresist mold is washed away after electroplating using acetone or photoresist strip remover. The seed layer is then removed by wet chemical etching. 
(a)

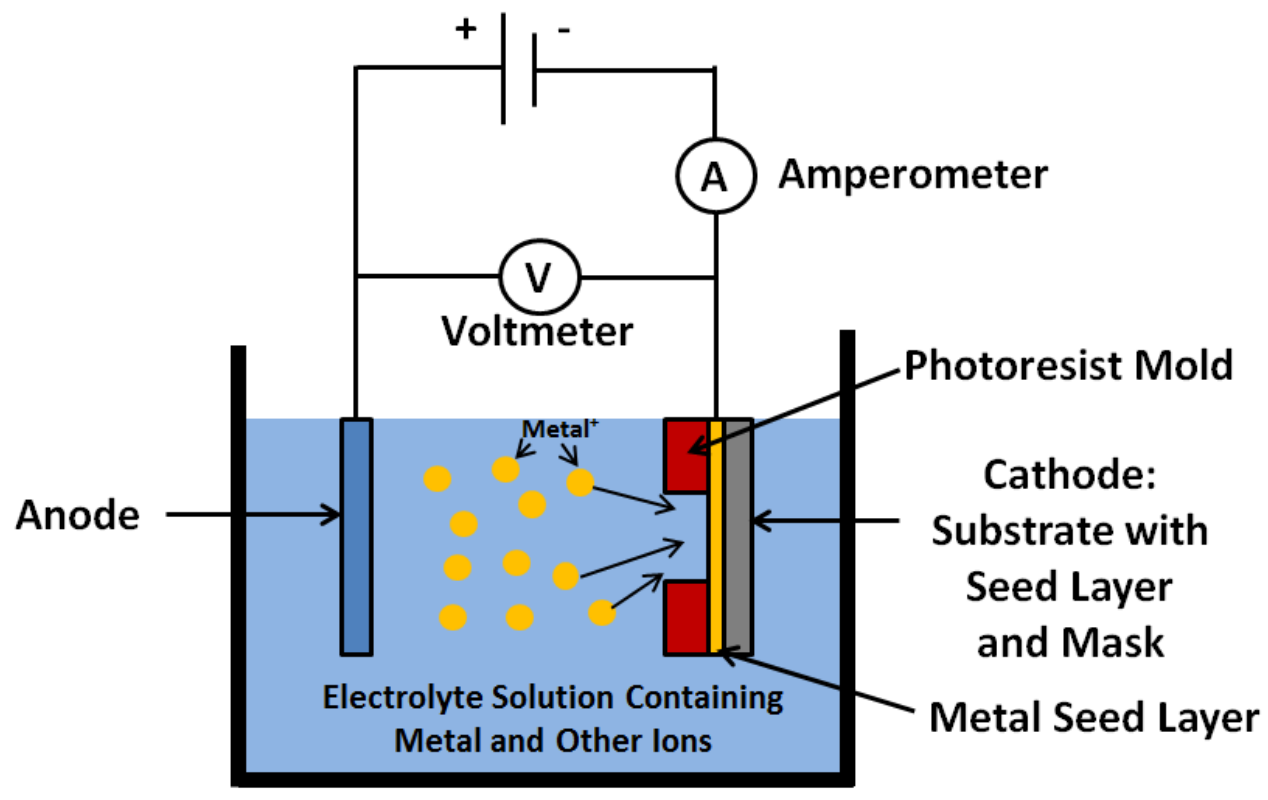

(b)

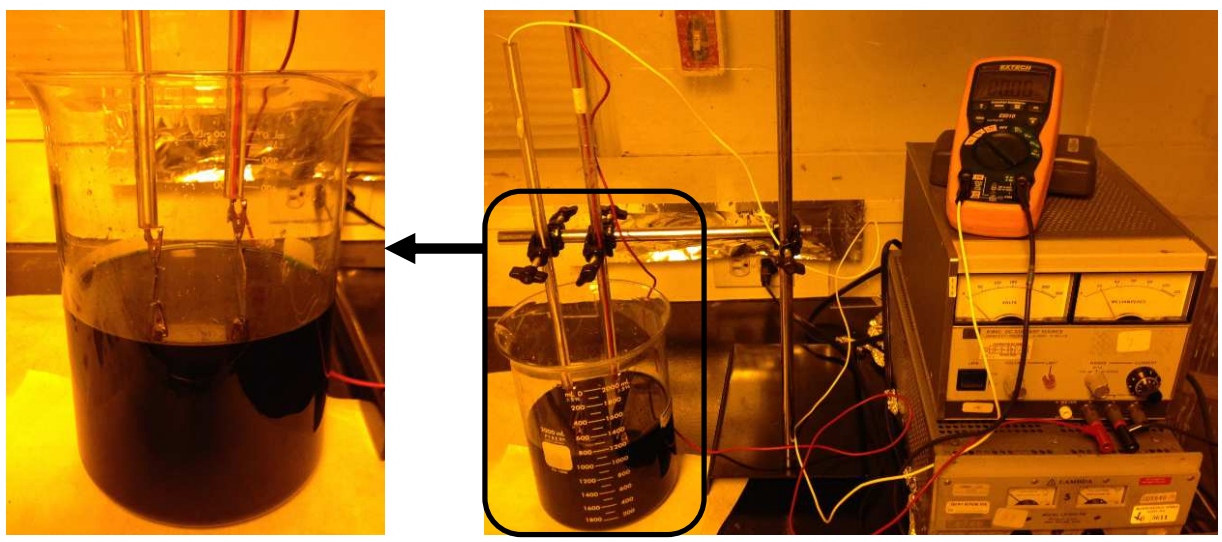

(c)

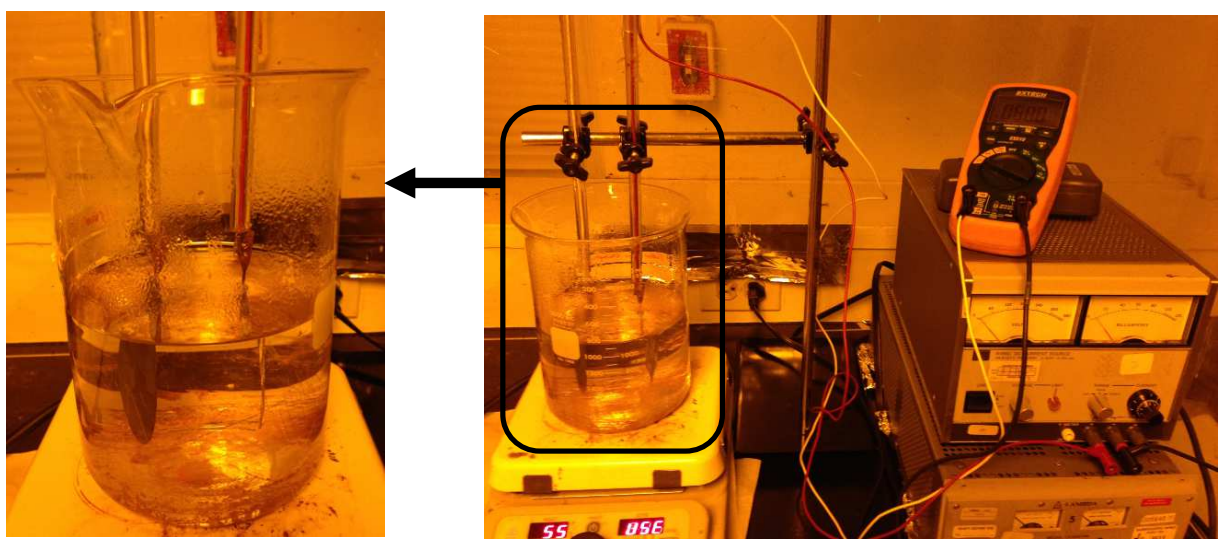

Figure 4.6. A schematic and photos of the experimental setups used for $\mathrm{Ni}$ and $\mathrm{Au}$ electroplating, (a) the electroplating process, (b) $\mathrm{Ni}$ electroplating, and (c) $\mathrm{Au}$ electroplating. 


\section{F. Lift-off process}

Lift-off process, an alternate technique for wet chemical etching or reactive ion etching, is a common technique to pattern metal or dielectric films. Lift-off process is used to create structures of a target material on the surface of a substrate using a sacrificial layer, usually a photoresist layer. In the lift-off process as shown in Figure 4.7, an inverse photoresist pattern is first defined, and then it is followed with thin film deposition. Those portions of the metal film that are deposited on top of the photoresist

\section{Photolithographic Patterning of Photoresist Sacrificial Layer}

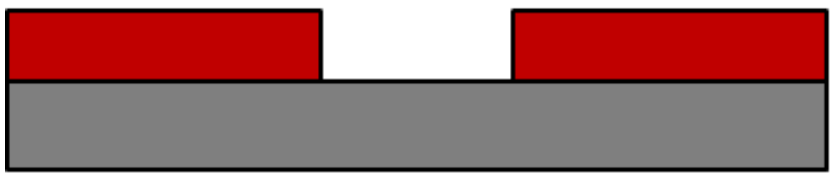

\section{Depositing Metal Film}

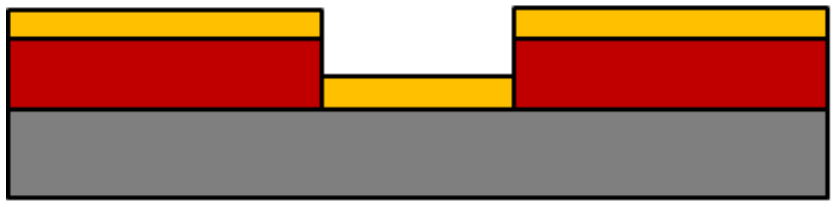

Photoresist dissolves away and liftoff the unwanted metal

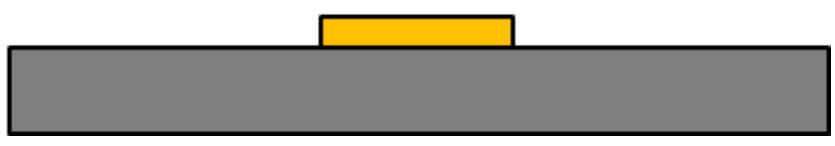

Figure 4.7. A standard Lift-off process for pattern generation using negative photoresist.

sacrificial layer are finally removed (lifted off) when the substrate is immersed in a suitable solvent, leaving behind the desired metal pattern. Negative photoresist is generally preferred for lift-off process because it helps reducing the thickness of the photoresist on the sidewalls, making the subsequent lift-off easier. In addition, the 
crosslinking of the resin of common negative photoresists can maintain the undercut at a high thin film deposition temperature, which helps to maintain the undercut.

\subsection{Fabrication process of MEMS variable capacitor with two-cavity}

The fabrication process of MEMS variable capacitor with two-cavity for power harvesting is based on surface micromachining technology using a set of seven quartz masks for defining the device structural layers' shapes. The details of fabrication process are described step by step below.

\subsubsection{Fabrication of masks}

The layouts of the masks were first drawn using L-Edit CAD tool. The layouts of the masks were then printed on a high resolution transparency which is $0.39 \mu \mathrm{m}$ per grid using service from an external company. The patterns on the transparency were then transferred to 4 inch square chromium-on-soda lime hard masks which are made of quartz glass with a chromium layer and an AZ-1518 photoresist layer. The patterns on the transparency were transferred by exposing the AZ-1518 photoresist layer under UV light with an intensity of $14 \mathrm{~mW} / \mathrm{cm}^{2}$ for 3 seconds in the photolithography process. The patterned AZ-1518 photoresist layer served as etching mask in chromium wet etching process to finally create the layouts on the chromium layer. The quartz hard masks with the chromium layouts were then used in the mask aligner for photolithography process.

The devices' layouts (two different designs) are distributed across the quartz masks such that an area of 3 inch can be utilized for the purpose of using 3 " silicon wafer as shown in Figure 4.8. The devices' layouts consist of seven different layers (masks): (1) The first mask is used twice to pattern square trenches at locations corresponding to the 


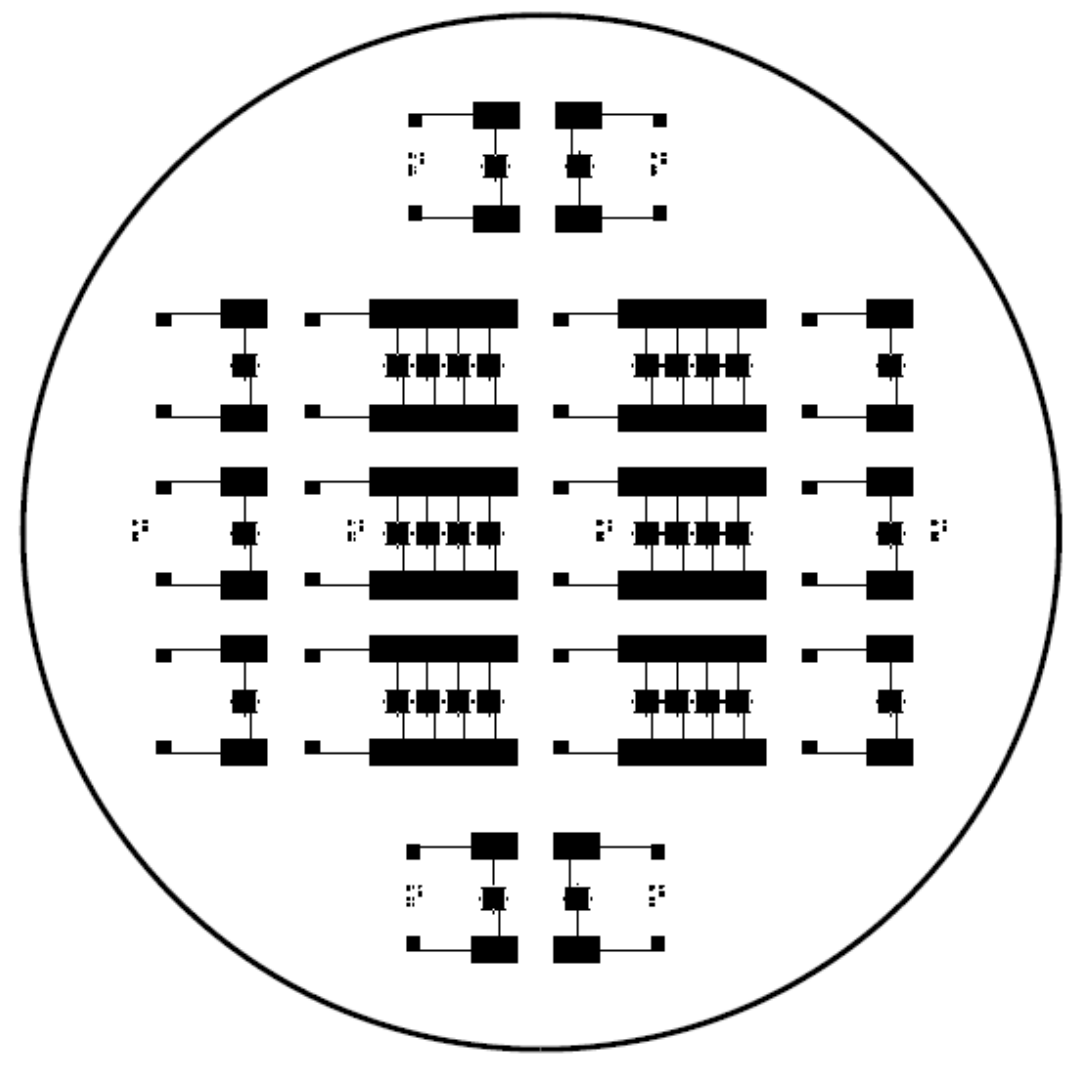

Figure 4.8. Arrangement of layouts of design 1 and design 2 on a 3" silicon wafer.

anchors of the movable plates and bonding tabs, and to pattern photoresist mold for electroplating the capacitors' anchors and bonding pads (Figure 4.9(a)). (2) The second mask is used to pattern the fixed plates, electrodes, trace lines, and the seed layer at locations corresponding to the capacitors' anchors and bonding tabs (Figure 4.9(b)). (3) The third mask is used to pattern the photoresist mold for electroplating the stoppers on the fixed plates (Figure 4.9(c)). (4) The fourth mask is used to pattern the sputter deposited silicon dioxide and silicon nitride dielectric layers on fixed plates (Figure 4.9(d)). (5) The fifth mask is used to pattern the photoresist mold for electroplating the movable plates and serpentine suspension beams (Figure 4.9(e) and 4.9(f)). (6) The sixth mask is used to pattern the photoresist mold for electroplating only at the movable plates 
(a)

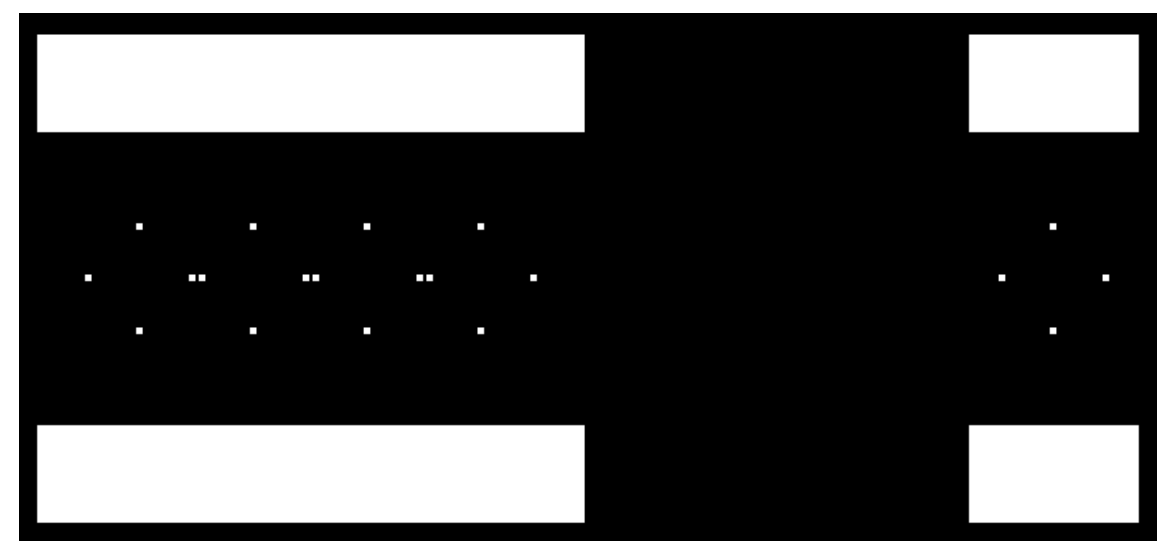

(b)
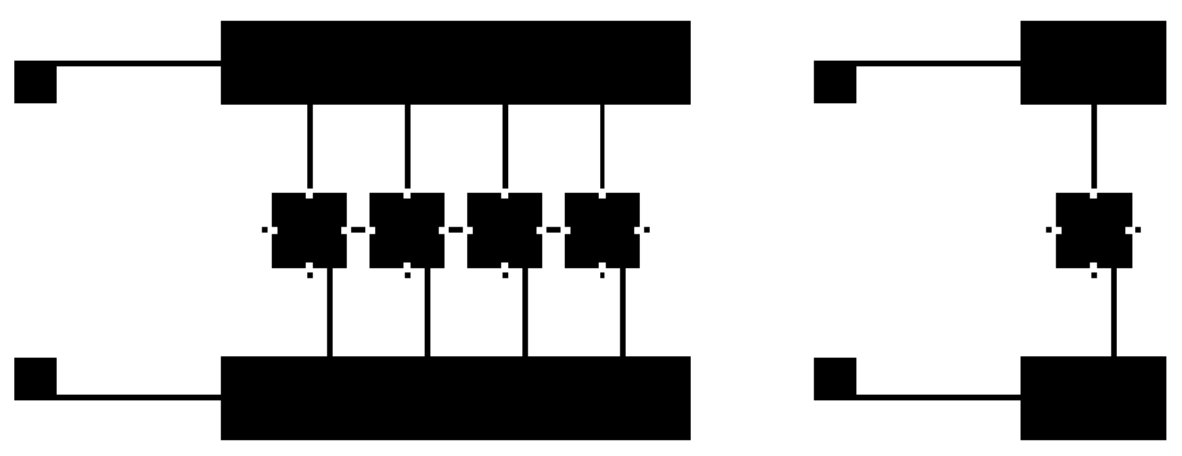

(c)
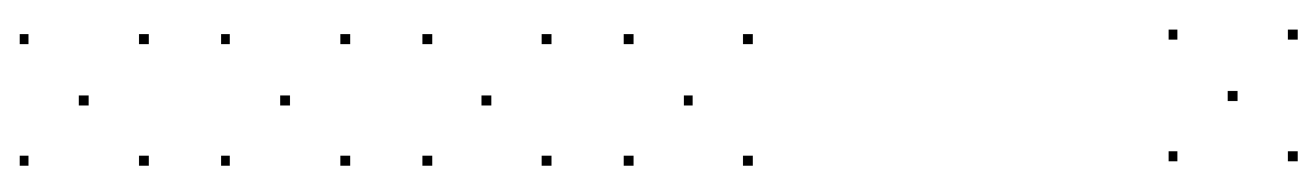

(d)
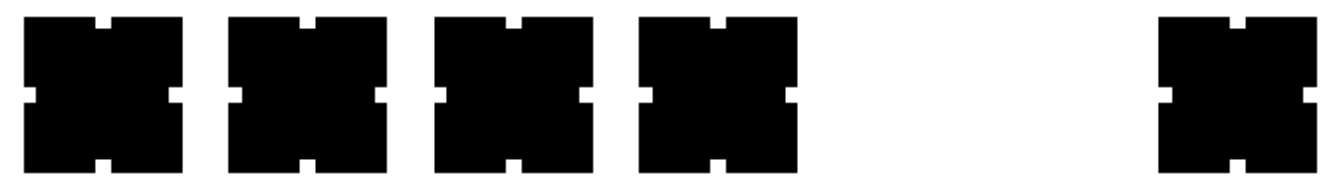

(e)

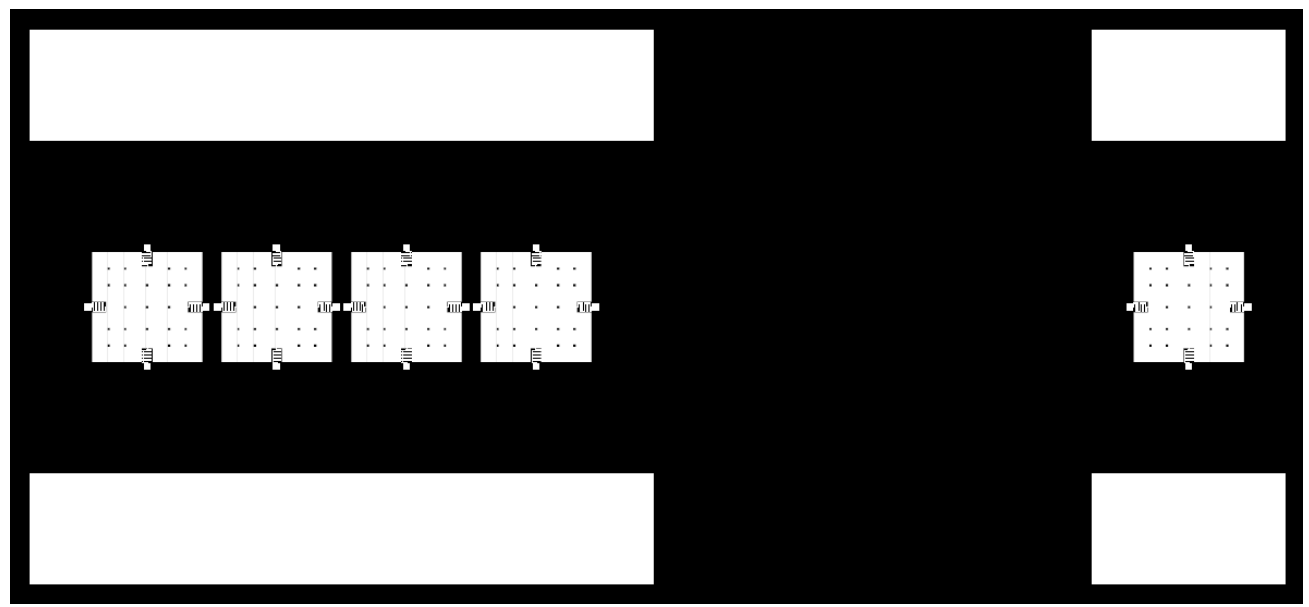


(f)

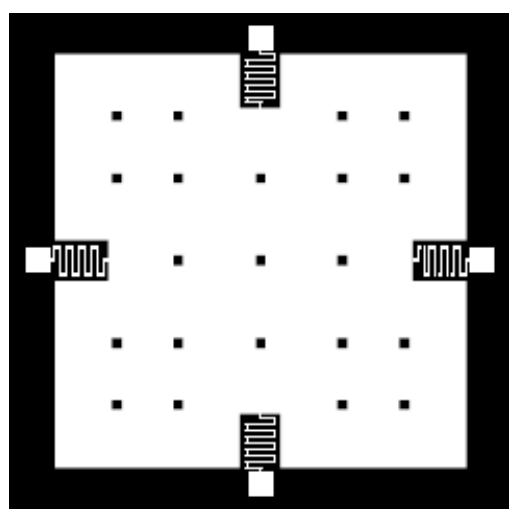

(g)

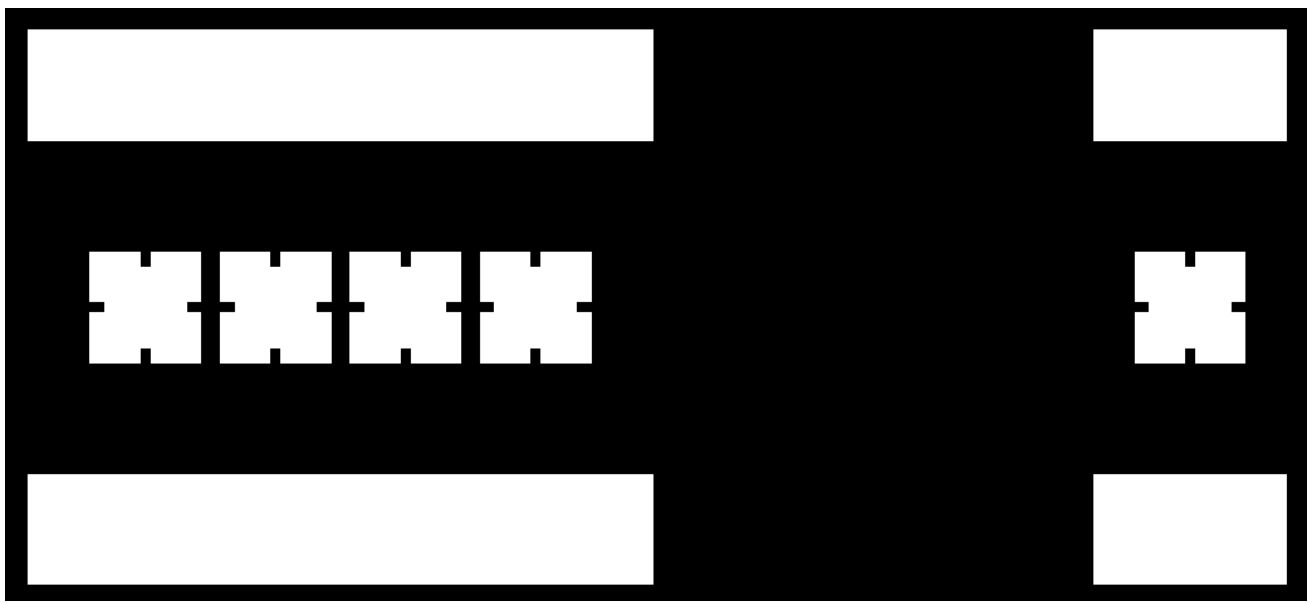

(h)

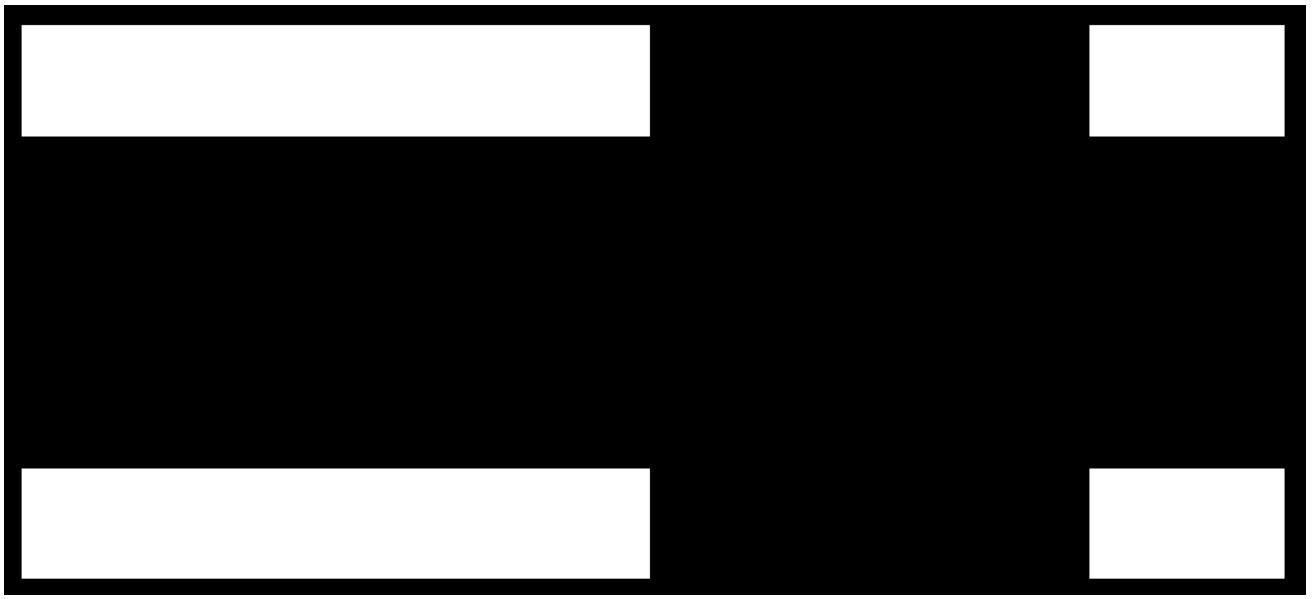

Figure 4.9. The layouts of design 1 and design 2 on the seven masks, (a) mask of anchors and bonding tabs, (b) mask of fixed plates, (c) mask of stoppers, (d) mask of dielectrics, (e) mask of movable plate with suspension beams, (f) magnified layout of movable plate with suspension beams, (g) mask of movable plate, (h) mask of bonding tabs. 
thus increasing the thickness of the movable plate without changing the thickness of serpentine suspension beams (Figure 4.9(g)). (7) The seventh mask is used to pattern the photoresist mold for electroplating thin indium layer on the bonding tads (Figure 4.9(h)). The seven masks introduced above are summarized in the table 8 .

Table 8. The seven masks set used in the fabrication process of MEMS variable capacitor with two-cavity.

\begin{tabular}{|l|c|c|}
\hline & Name & Descriptions \\
\hline The mask 1 & $\begin{array}{c}\text { Anchors and bonding tabs } \\
\text { mask }\end{array}$ & $\begin{array}{c}\text { To create the patterns of anchors and } \\
\text { bonding tabs }\end{array}$ \\
\hline The mask 2 & Fixed plates mask & $\begin{array}{c}\text { To create the patterns of fixed plates, } \\
\text { electrodes, trace lines, and the seed } \\
\text { layers of anchors and bonding tabs }\end{array}$ \\
\hline The mask 3 & Stoppers mask & $\begin{array}{c}\text { To create the photoresist mold for } \\
\text { electroplating the stoppers }\end{array}$ \\
\hline The mask 4 & Dielectric mask & $\begin{array}{c}\text { To pattern the dielectric layers } \\
\text { To create the photoresist mold for } \\
\text { electroplating the movable plate with } \\
\text { suspension beams }\end{array}$ \\
\hline The mask 6 & $\begin{array}{c}\text { Movable plate with } \\
\text { suspension beams mask }\end{array}$ & $\begin{array}{c}\text { To pattern the photoresist mold for } \\
\text { electroplating only the movable plates }\end{array}$ \\
\hline The mask 7 & Bonding tabs mask & $\begin{array}{c}\text { To pattern the photoresist mold for } \\
\text { electroplating indium bonding layer }\end{array}$ \\
\hline
\end{tabular}

4.2.2 Fabrication of the device with a movable plate and suspension beams thickness of $30 \mu \mathrm{m}$ and $5 \mu \mathrm{m}$

The MEMS variable capacitors with two-cavity were fabricated using surface micromachining technologies on top of 3" silicon wafers using the following sequence of steps: 
(1) The silicon wafer is first cleaned using piranha solution which is prepared by adding 50\% concentration hydrogen peroxide $\left(\mathrm{H}_{2} \mathrm{O}_{2}\right)$ into $98 \%$ concentration sulfuric acid $\left(\mathrm{H}_{2} \mathrm{SO}_{4}\right)$ at the ratio of $\mathrm{H}_{2} \mathrm{SO}_{4}: \mathrm{H}_{2} \mathrm{O}_{2}=3: 1$. The wafer is then immersed in the piranha solution bath for 5 minutes for removing the organic contaminations. This is followed with DI water rinsing. The natural oxide layer is then etched using Hydrofluoric acid $(\mathrm{HF})$ diluted with DI water (1: 10 ratio). The time needed to remove this layer is between 1 to 1:30 minutes. The wafer is then rinsed with DI water for two minutes to remove the residue chemicals.

(a)
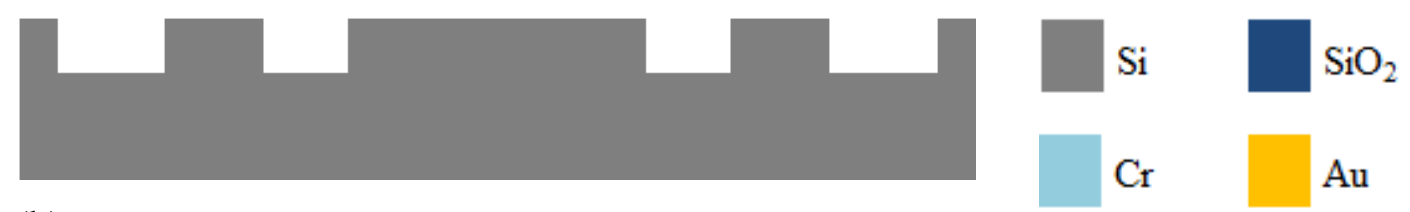

(b)
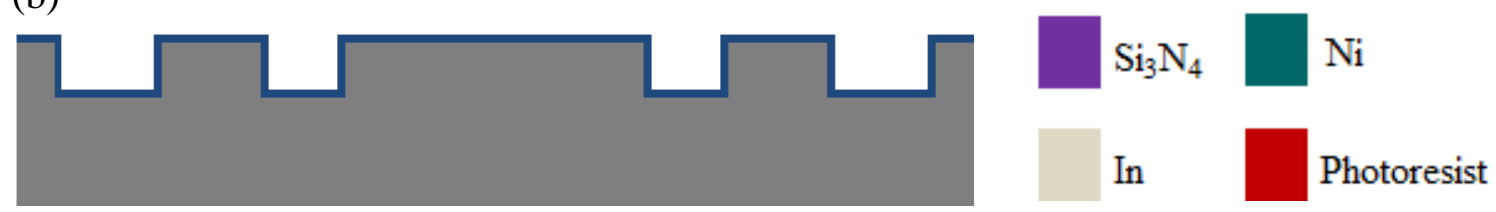

(c)

(d)
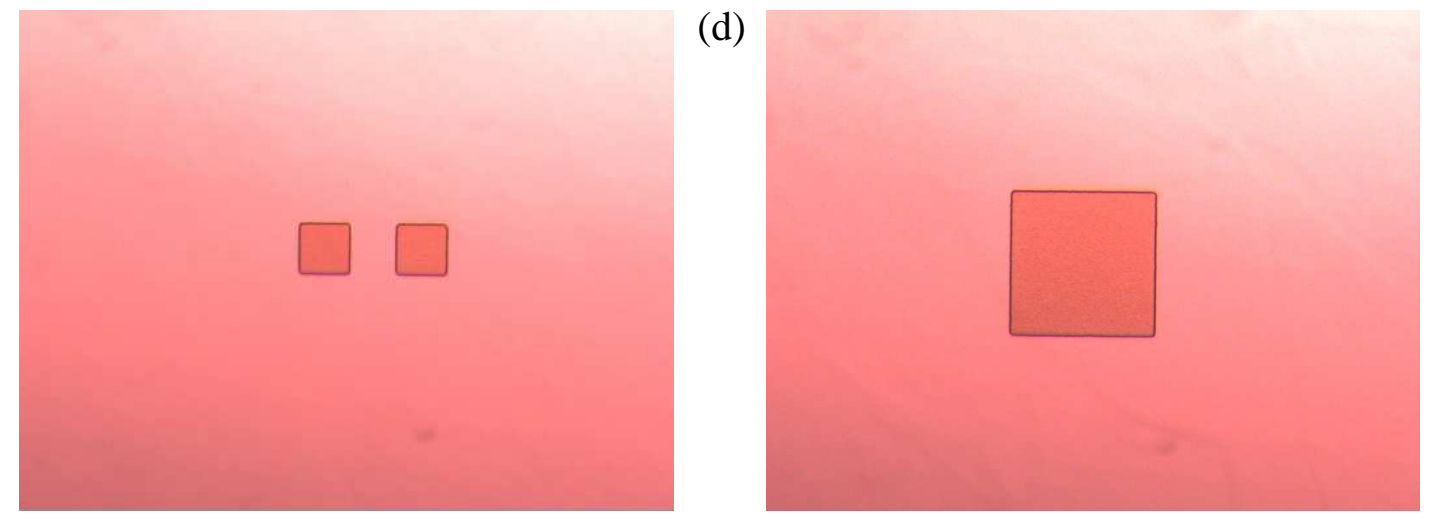

Figure 4.10. (a) The sideview schematic shows the substrate cleaning and RIE etching for anchors and bonding tabs, (b) the sideview schematic shows the thermal oxidation to grow the insulation layer, (c) optical pictures of the square trenches for anchors after thermal oxidation, (d) a magnified image of the square trench for an anchor. 
(2) Immediately after the wafer cleaning, a thin layer of Shipley 1813 photoresist is spin coated and patterned by photolithography using the mask of anchors and bonding tabs. Rectangular trenches corresponding to the capacitor anchors and bonding tabs are etched into the silicon wafer with a depth of $3 \mu \mathrm{m}$ using RIE system. The RIE etching of silicon uses $\mathrm{SF}_{6}$ and $\mathrm{O}_{2}$ gases, with flow rates of $50 \mathrm{sccm}$ and $10 \mathrm{sccm}$, respectively, at a RF power of $100 \mathrm{~W}$, which takes around 5 minutes to etch $3 \mu \mathrm{m}$. The photoresist layer is then removed. The silicon wafer is then thermally oxidized at $1100{ }^{\circ} \mathrm{C}$ using high temperature furnace to grow a $\mathrm{SiO}_{2}$ layer with a thickness of $1 \mu \mathrm{m}$ for insulation (see Figure 4.10). The sideview fabrication schematics and optical pictures of the square trenches after thermal oxidation are shown in Figure 4.10.

(3) Thin layers of Chromium (Cr) and Gold (Au), with a thickness of $50 \mathrm{~nm}$ and $200 \mathrm{~nm}$, respectively, are deposited using DC magnetron sputtering in Kurt Lesker AXXIS Multi-Deposition System. The deposition parameters include a chamber base pressure of $1 \times 10^{-7}$ Torr or lower, deposition pressure of 4 mTorr achieved by argon flows into the vacuum chamber, and a DC power of $200 \mathrm{~W}$ and $90 \mathrm{~W}$ are used for Cr and $\mathrm{Au}$ depositions, respectively. The deposition durations are 5 minutes and 7 minutes for $\mathrm{Cr}$ and $\mathrm{Au}$, respectively (see Figure 4.11(c) and 4.11(d)). A thin layer of Shipley 1813 photoresist is similarly patterned using the mask of fixed plates. The Au layer is wet etched in potassium iodide $(\mathrm{KI})$ and iodine $\left(\mathrm{I}_{2}\right)$ solution using the patterned photoresist as etching mask. The patterned Au layer forms the fixed plates, electrodes, trace lines, and the seed layer for the capacitor anchors and bonding tabs as shown in Figure 4.11(e) and 4.11(f). The Shipley 1813 photoresist is removed by acetone after wet etching of Au. The sideview fabrication schematics of this step are shown in the figure 4.11(a) and 4.11(b). 
(a)
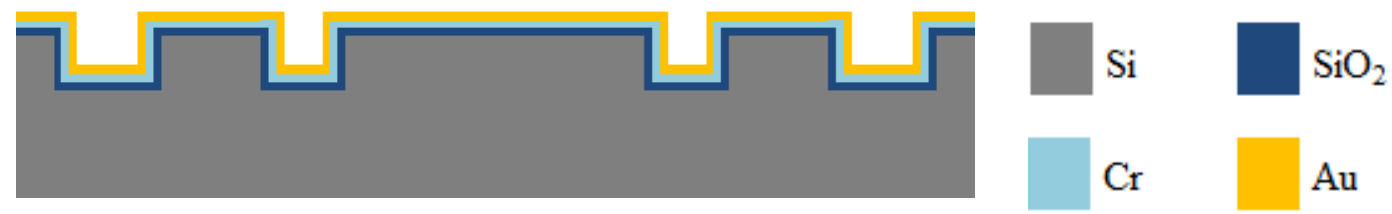

(b)
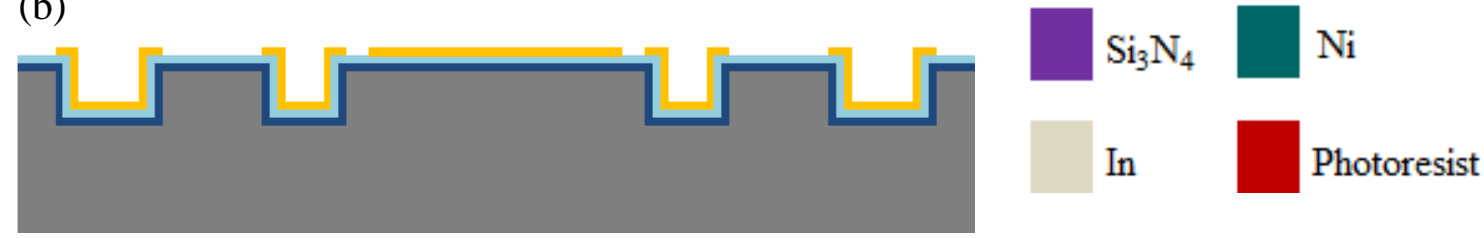

(c)

(d)
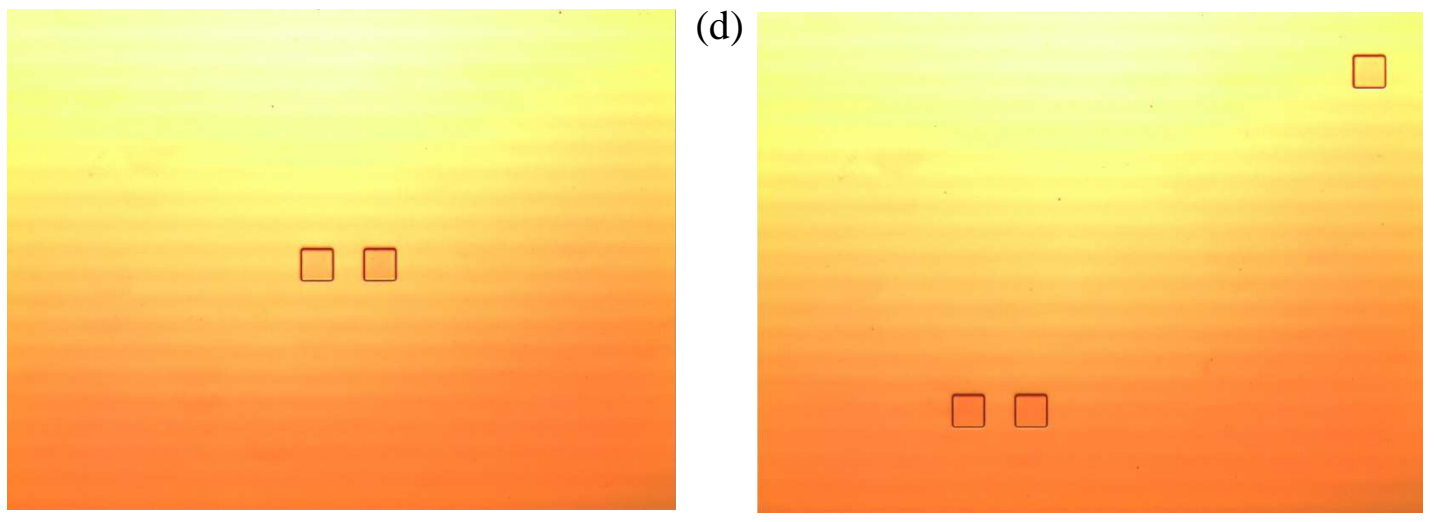

(e)

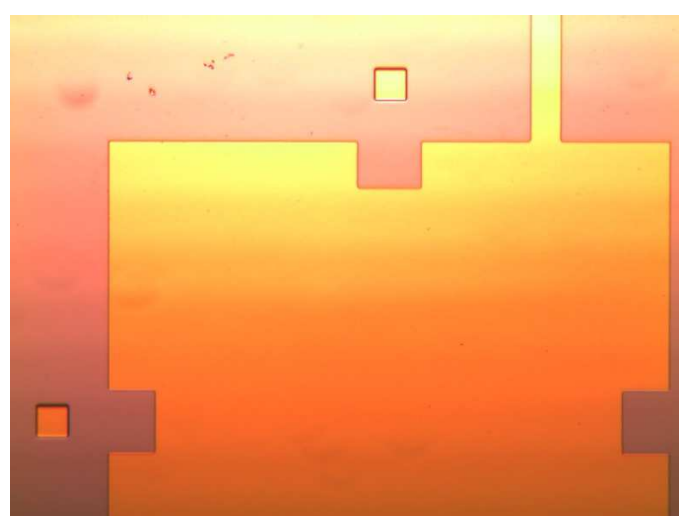

(f)

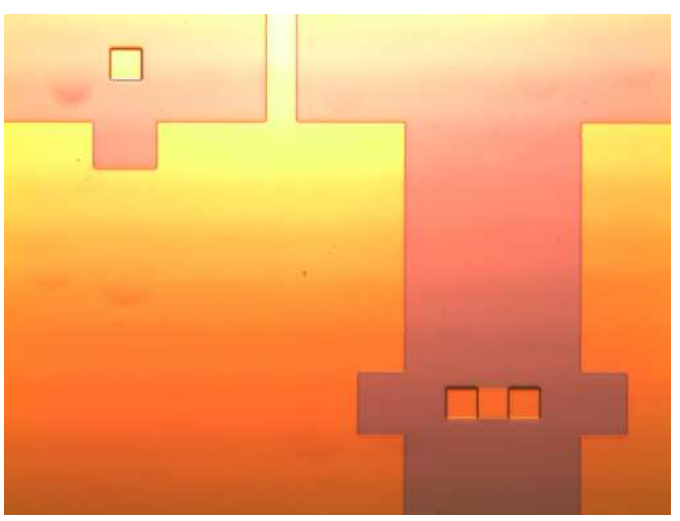

Figure 4.11. (a) The sideview schematic shows the $\mathrm{Cr}$ and $\mathrm{Au}$ thin films are sputter deposited, (b) the sideview schematic shows the Au thin film patterning for bottom plates, trace lines and seed layer for anchors and bonding tabs, (c) \& (d) optical pictures of the square trenches for anchors after $\mathrm{Cr}$ and Au sputter depositions, (e) \& (f) optical pictures of patterned fixed plates, anchors and trace lines. 
(4) An AZ-P4620 photoresist layer with a thickness of $10 \mu \mathrm{m}$ is patterned using photolithography with the mask of stoppers. The stoppers are formed by electroplating $\mathrm{Au}$ for a thickness of $4 \mu \mathrm{m}$ inside the photoresist mold using Technic gold 25 ES solution. The electroplating parameters including deposition rate and thickness were calibrated and measured using KLA-Tencor P-16+ profiler-meter. The AZ-P4620 photoresist mold is then removed after the electroplating. The electroplated Au stoppers are shown in Figure 4.12(c) and 4.12(d). A layer of NRP-3000PY photoresist is then patterned by photolithography using the mask of dielectrics. Two thin layers of $\mathrm{SiO}_{2}$ and $\mathrm{Si}_{3} \mathrm{~N}_{4}$, both with a thickness of $30 \mathrm{~nm}$, are deposited on the NRP-3000PY photoresist layer using RF magnetron sputtering in Kurt Lesker AXXIS Multi-Deposition System at a power of 200 W. It should be noted that the $\mathrm{SiO}_{2}$ and $\mathrm{Si}_{3} \mathrm{~N}_{4}$ can only be sputtered using RF power because the $\mathrm{SiO}_{2}$ and $\mathrm{Si}_{3} \mathrm{~N}_{4}$ target will cumulate charge on its surface if using DC magnetron sputtering which will rise a reverse potential against the applied DC power thus lowering or stopping the sputtering process. The dielectric layers of $\mathrm{SiO}_{2}$ and $\mathrm{Si}_{3} \mathrm{~N}_{4}$ are then defined on the fixed plates by lifting off the NRP-3000PY photoresist layer in an acetone bath with ultrasonic agitation. Figure 4.12(e) and 4.12(f) show a fixed plate with dielectric layers on its top (the color of the plates' surface become purple comparing with Figure 4.12(c) and 4.12(d)). The sideview fabrication schematics of this step are shown in Figure 4.12(a) and 4.12(b).

(5) An AZ-P4620 photoresist layer with a thickness of $30 \mu \mathrm{m}$ is created by double spin coating which is simply referred to a process of spin coating and soft baking the substrate twice with a low viscous photoresist to achieve the photoresist layer with a high thickness. The AZ-P4620 photoresist layer is then patterned to serve as a sacrificial layer 


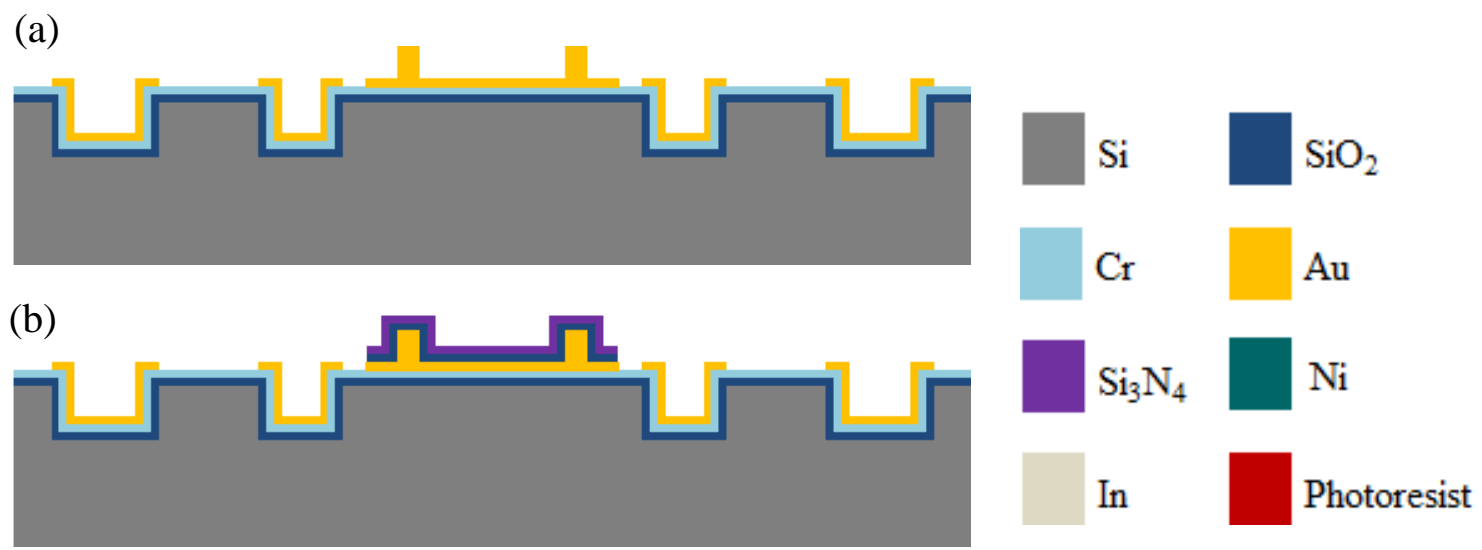

(c)

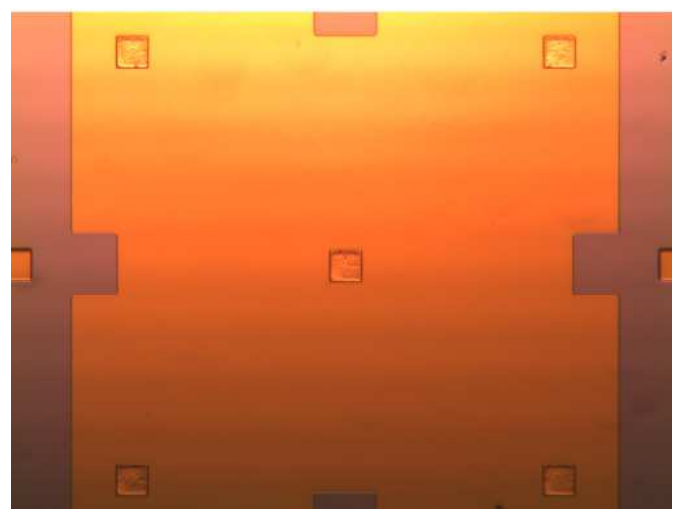

(e)

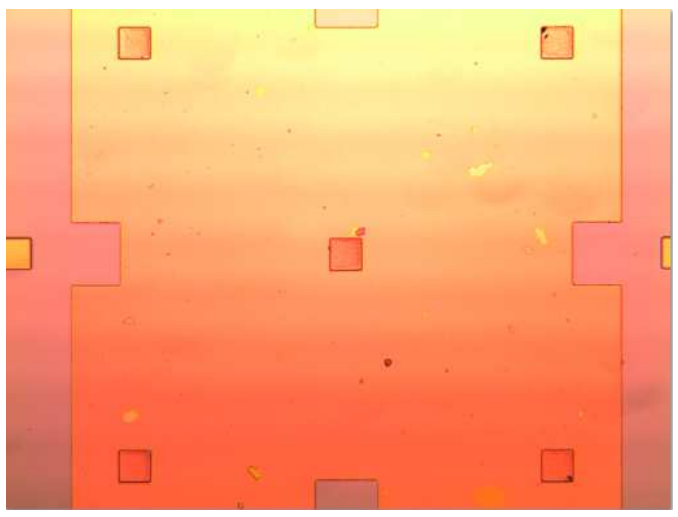

(d)

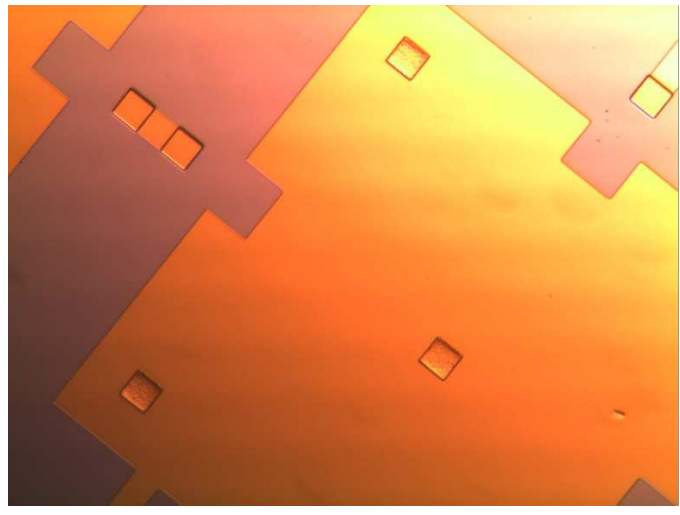

(f)

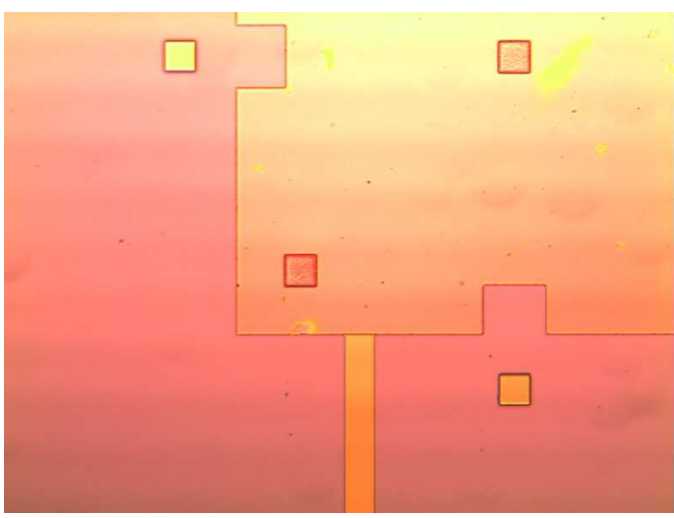

Figure 4.12. (a) The sideview schematic shows the electroplated Au stoppers, (b) the sideview schematic shows the sputtered and patterned $\mathrm{SiO}_{2}$ and $\mathrm{Si}_{3} \mathrm{~N}_{4}$ dielectric layers, (c) \& (d) optical pictures of the electroplated Au stoppers on the plates, (e) \& (f) optical pictures of the plates with $\mathrm{Au}$ stoppers after being covered by $\mathrm{SiO}_{2}$ and $\mathrm{Si}_{3} \mathrm{~N}_{4}$ dielectric layers. 
using photolithography with the mask of anchors and bonding tabs. The patterned photoresist sacrificial layer also serves as a mold for electroplating the capacitor anchors and bonding tabs. Nickel (Ni) is then electroplated for a thickness of $30 \mu \mathrm{m}$ inside the photoresist mold using "Technic High Speed Nickel Sulfamate FFP RTU" solution as shown in Figure 4.13(b) and 4.13(c). After the completion of $\mathrm{Ni}$ electroplating, the photoresist sacrificial layer is cured for proper time using an oven at an optimized temperature to prevent cracking and wrinkling in later steps. The sideview fabrication schematics of this step are shown in Figure 4.13(a).

(6) Two thin layers of $\mathrm{Cr}$ and $\mathrm{Au}$, with a thickness of $50 \mathrm{~nm}$ and $200 \mathrm{~nm}$, respectively, are deposited using the same DC magnetron sputtering process introduced

(a)
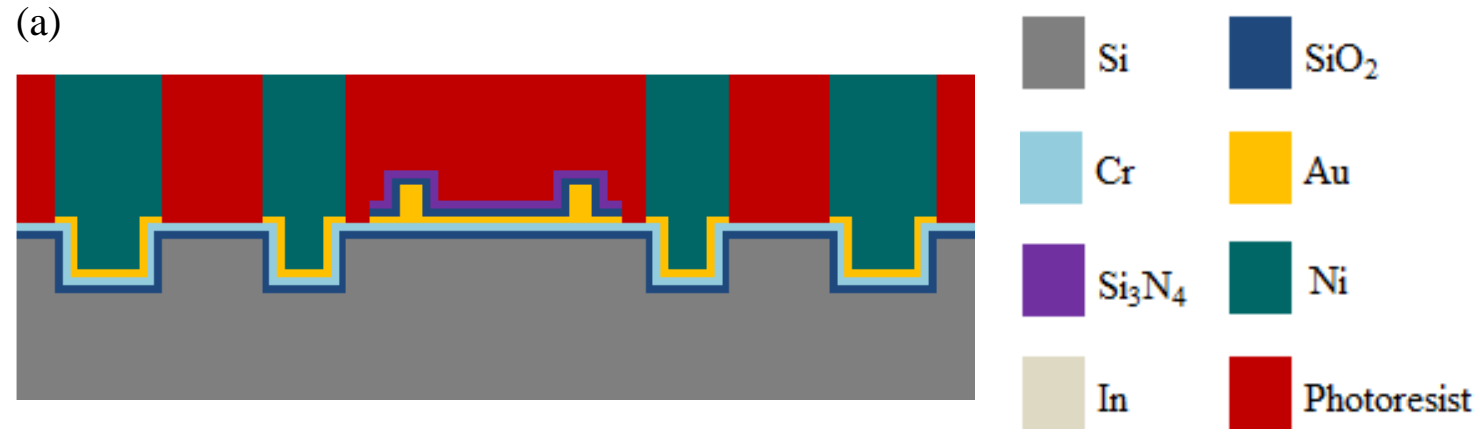

(b)

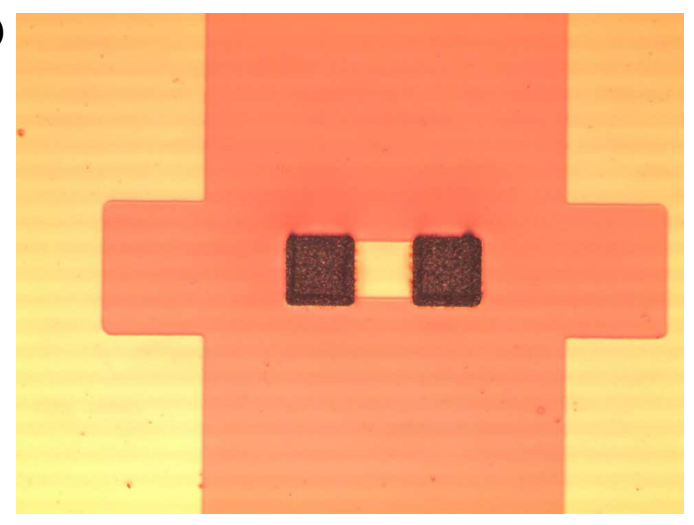

(c)

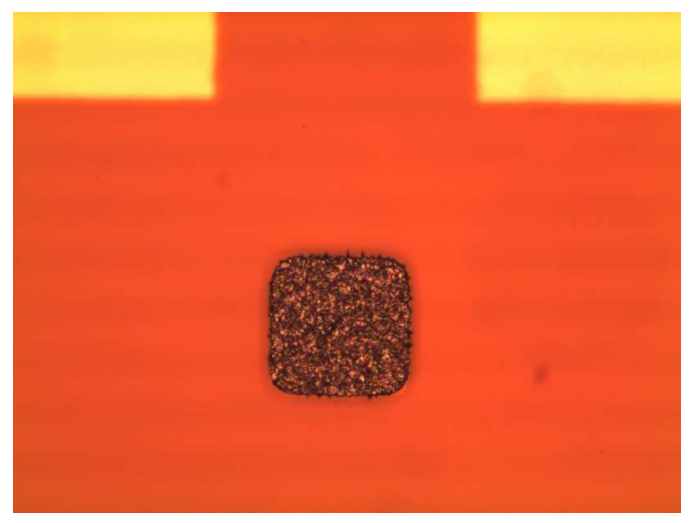

Figure 4.13. (a) The sideview schematic shows the Ni electroplating for anchors and bonding tabs, (c) optical pictures of the electroplated $\mathrm{Ni}$ anchors, (d) a magnified image of an electroplated $\mathrm{Ni}$ anchor. 
(a)

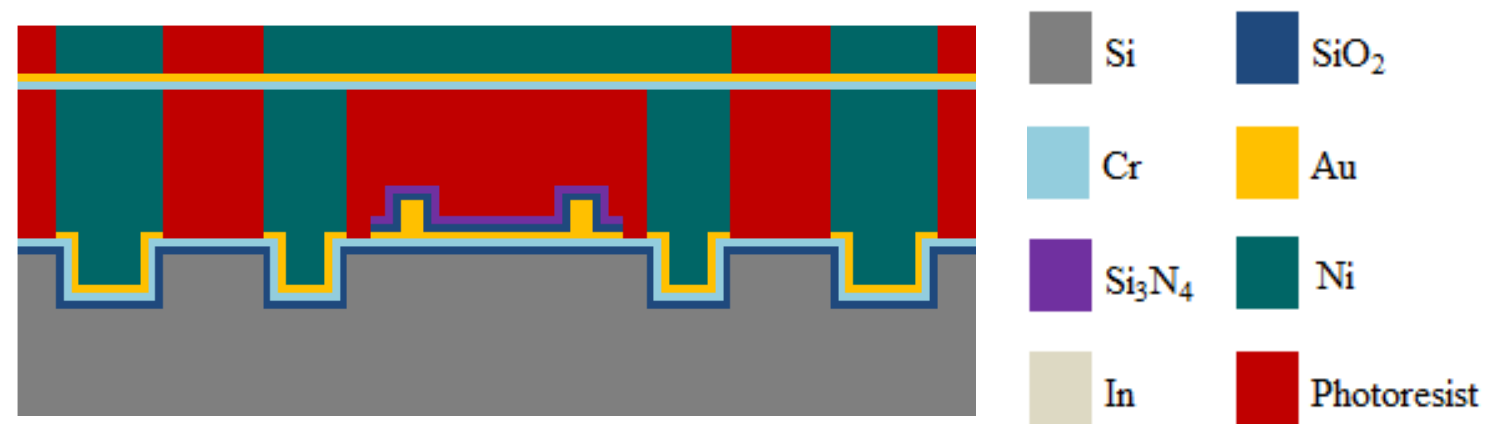

(b)

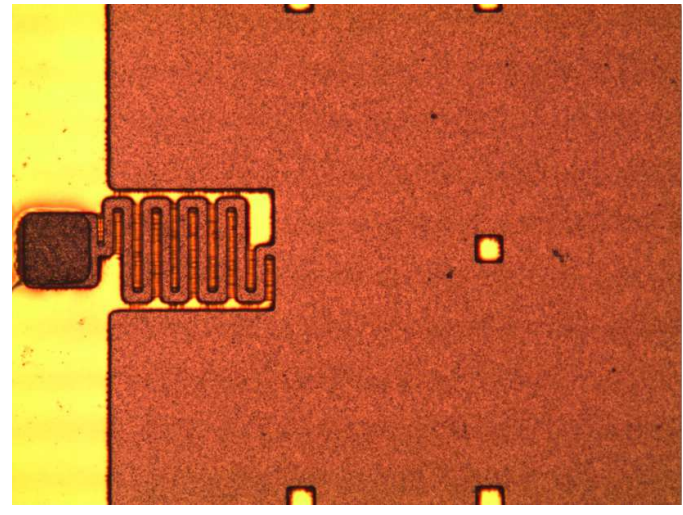

(c)

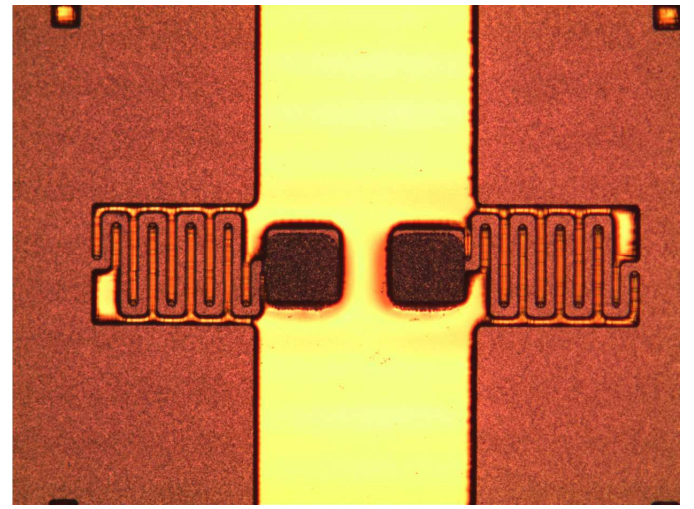

Figure 4.14. (a) The sideview schematic shows the sputter depositions of $\mathrm{Cr}$ and $\mathrm{Au}$ thin films for the second seed layer and forming movable plate with serpentine suspension beam by Ni electroplating, (b) \& (c) optical pictures of the Ni electroplated movable plate with serpentine suspension beams.

in the step (3). The $\mathrm{Cr}$ and $\mathrm{Au}$ layers are sputter deposited on top of sacrificial photoresist layer serving as a second seed layer for electroplating Ni. An AZ-P4620 photoresist layer with a thickness of $10 \mu \mathrm{m}$ is patterned using photolithography with the mask of movable plate with suspension beams. The movable plates with serpentine suspension beams are created by electroplating $\mathrm{Ni}$ for a thickness of $5 \mu \mathrm{m}$ inside the photoresist mold using the same Ni electroplating process introduced in the step (5). The sideview fabrication schematics of this step are shown in Figure 4.14(a). The optical pictures of $\mathrm{Ni}$ electroplated movable plate with serpentine suspension beams are shown in Figure 4.14(b) 
and 4.14(c).

(7) Another AZ-P4620 photoresist layer with a thickness of $20 \mu \mathrm{m}$ is added on the previous layer and patterned using photolithography with the mask of movable plate. Ni is electroplated inside of the photoresist mold using the same Ni electroplating process to increase the total thickness of movable plate up to $30 \mu \mathrm{m}$ without changing the thickness of serpentine suspension beams. The sideview fabrication schematic of this step is shown in Figure 4.15(a). The main structure of MEMS variable capacitor before bonding the top cavity is now completed as shown in Figure 4.15(b) and 4.15(c).

(a)
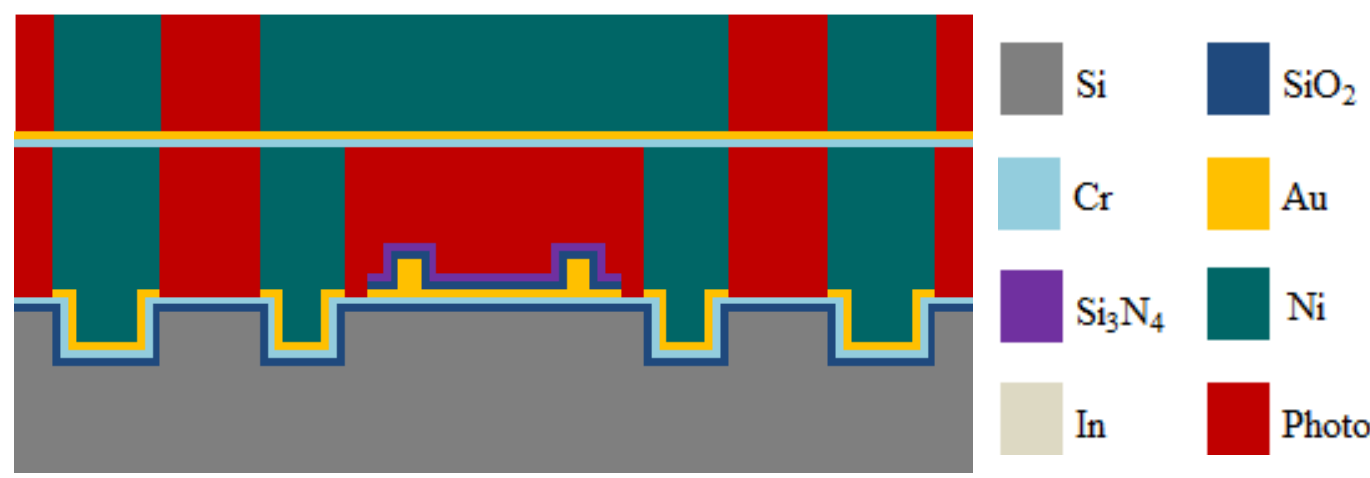

(b)

(c)
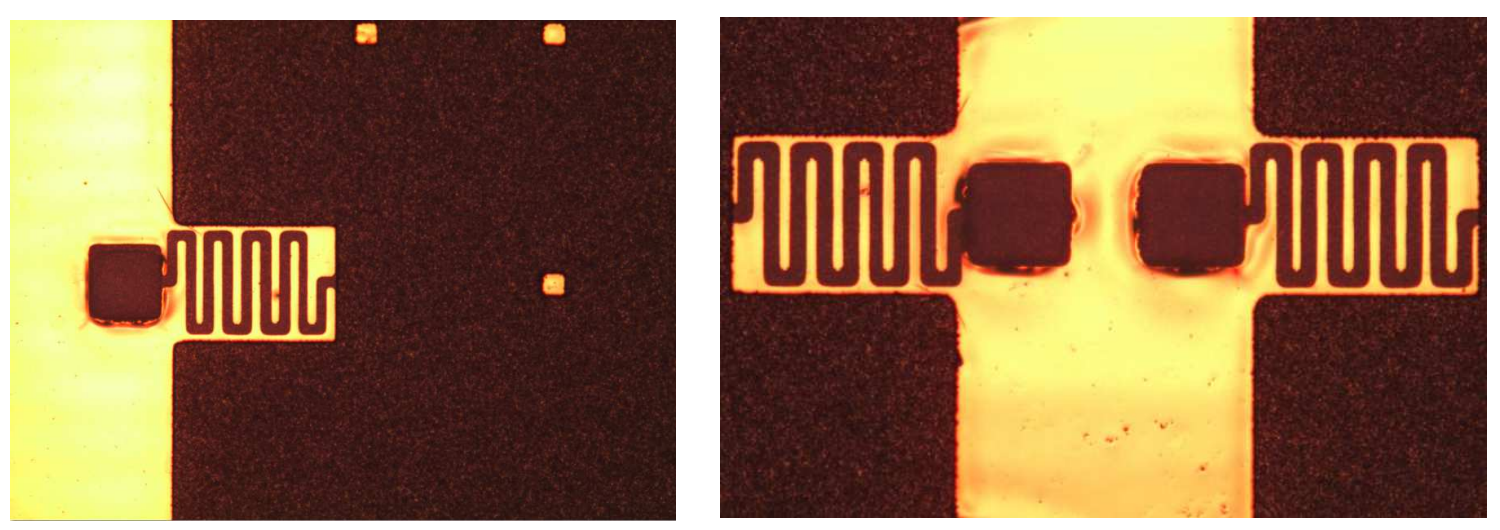

Figure 4.15. (a) The sideview schematic shows the Ni electroplating to increase the thickness of the movable plate, (b) \& (c) optical pictures of the movable plate with increased thickness, and without changing the thickness of suspension beams. 
(8) All AZ-P4620 photoresist layers on top of the second seed layer are then removed by flushing the acetone, isopropanol and DI water very carefully without attacking the sacrificial layer. The second seed layers of $\mathrm{Cr}$ and $\mathrm{Au}$ are removed by $\mathrm{Cr}$ and Au etchants. The AZ-P4620 photoresist sacrificial layer is then released in a hot AZ photoresist stripper bath at $95{ }^{\circ} \mathrm{C}$. The ultrasonic agitation is added to help the removal of the photoresist sacrificial layer under the movable plates. The first seed layer of $\mathrm{Cr}$ on the silicon substrate is finally etched away. The sideview fabrication schematic of this step is shown in Figure 4.16(a). The optical pictures of released movable plates with suspension beams are shown in Figure 4.16(b) and 4.16(c).

(a)
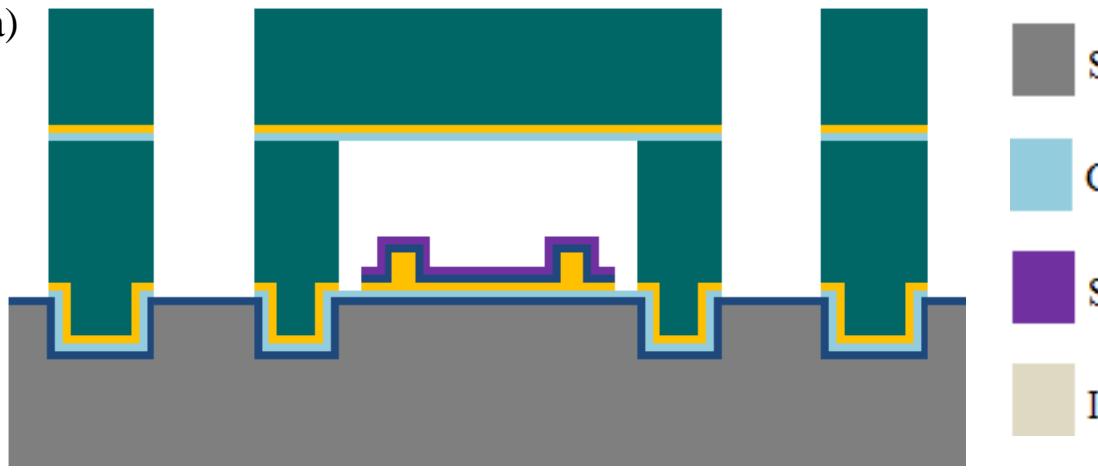

$\mathrm{Si}$
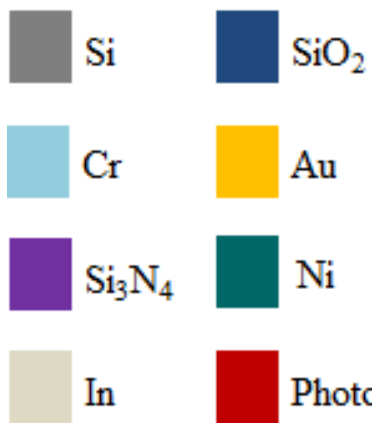

$\mathrm{Si}_{3} \mathrm{~N}_{4}$

In

Photoresist

(b)

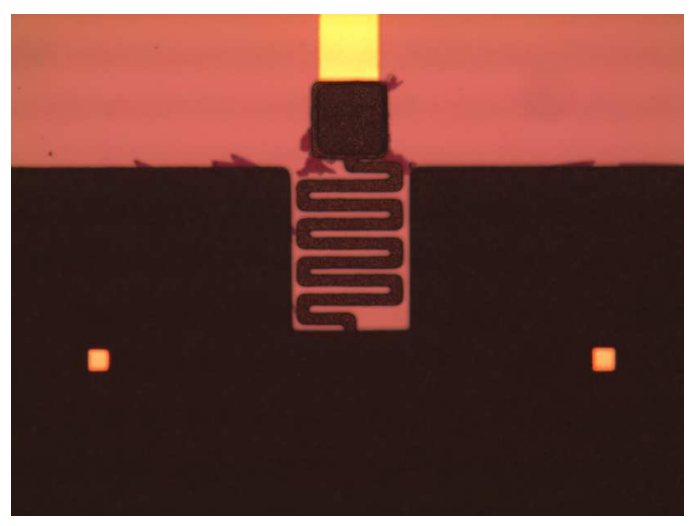

(c)

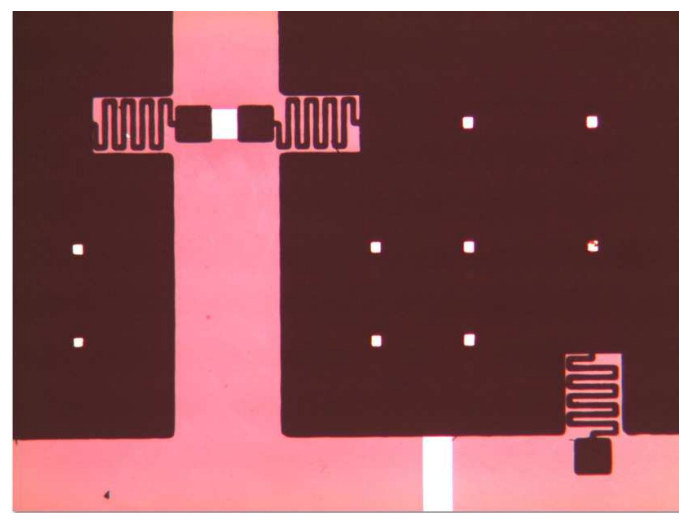

Figure 4.16. (a) The sideview schematic shows the releasing all photoresist layers, (b) \& (c) optical pictures of the movable plate with suspension beams after photoresist releasing. 
(9) The second silicon substrate only with the fixed plates, stoppers and dielectric layers are prepared using the same fabrication process as described previously. Instead of using the mask of anchors and bonding tabs, the AZ-P4620 photoresist sacrificial layer is patterned to an electroplating mold for bonding tabs using the mask of bonding tabs. The bonding tabs are similarly formed by electroplating $\mathrm{Ni}$ for a thickness of $30 \mu \mathrm{m}$ inside the mold. In addition, a thin layer of indium (In) about $1 \mu \mathrm{m}$ was electroplated on top of the bonding tabs. The substrate as shown in the sideview fabrication schematic of Figure 4.17(a) is used to create the top capacitive cavity.

(a)

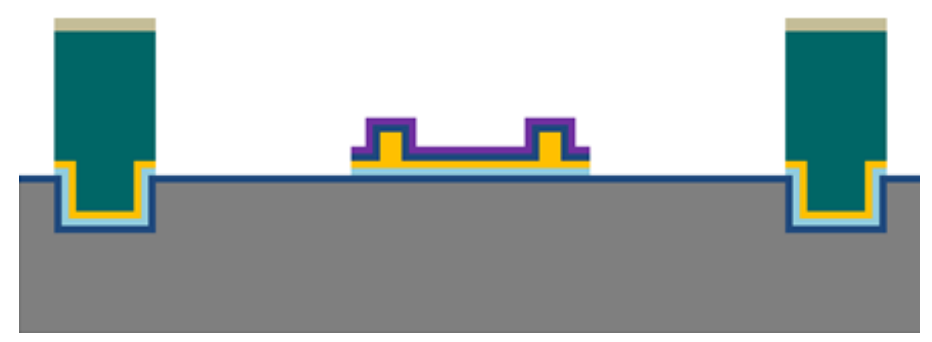

(b)

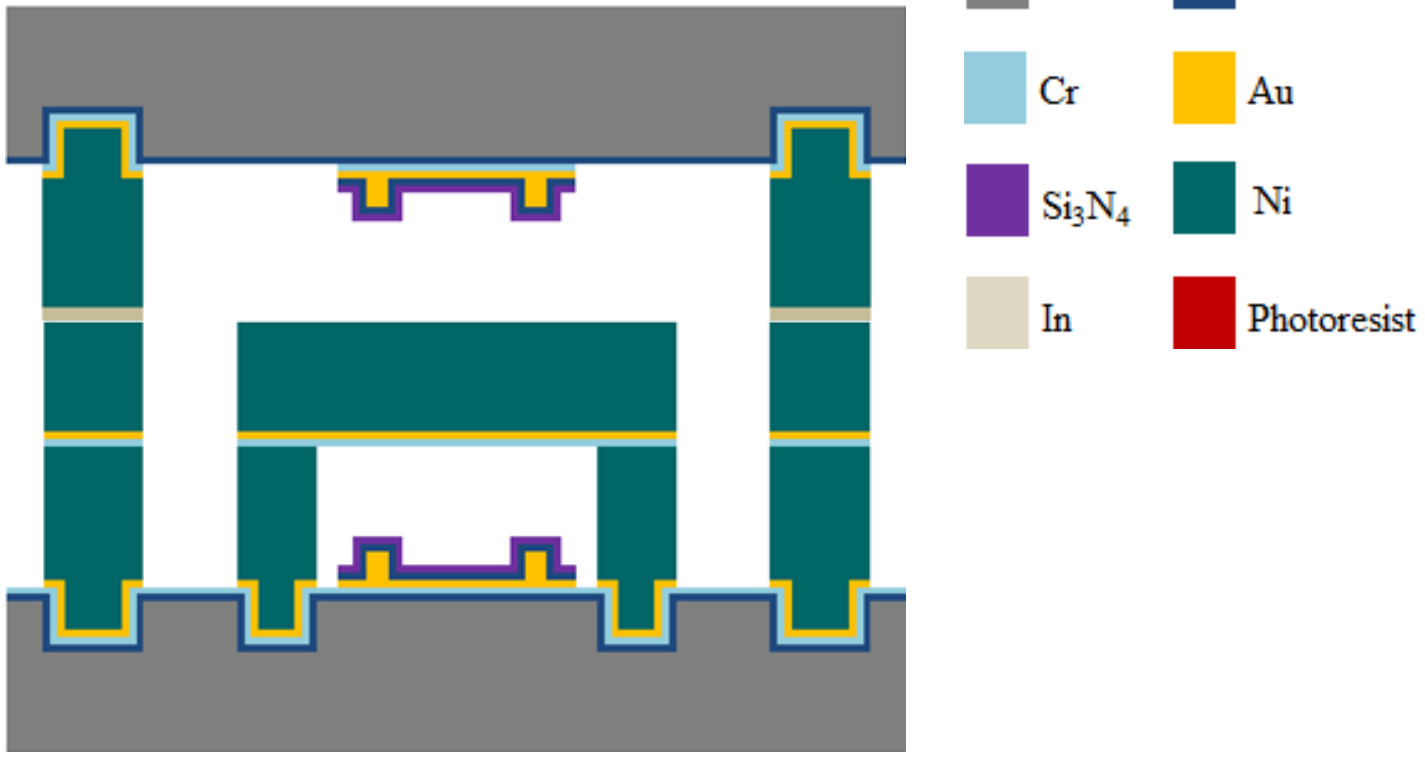

Figure 4.17. (a) The sideview schematic shows the fabricating top substrate with In bonding layer, (b) the sideview schematic shows the forming the final device by In bonding. 
(10) To finally create the two-cavity structure, the two substrates are diced into small dies, each die contains one device. The devices are then placed on top of a hotplate. The bonding tabs on each device are aligned under the microscope. Once the two dies are aligned, they were heated at $200{ }^{\circ} \mathrm{C}$ in order to soften the two indium layers and form a strong bond between them when the devices are cooled down. This temperature was found to give the best bonding quality without affecting the structure integrity. The sideview fabrication schematic of bonding the top cavity is shown in Figure 4.17(b).

The MEMS variable capacitors with two-cavity have been successfully fabricated using surface micromachining technologies described in above steps. The SEM micrographs of the devices with $30 \mu \mathrm{m}$ thick movable plate and $5 \mu \mathrm{m}$ thick suspension beams are shown in Figure 4.18. The movable plate with an area of $2 \times 2 \mathrm{~mm}^{2}$ and a thickness of $30 \mu \mathrm{m}$ is well suspended by the $5 \mu \mathrm{m}$ thick suspension beams and $30 \mu \mathrm{m}$ height anchors as shown in Figure 4.18(a). The serpentine suspension beams were made with a width, thickness and total length (four serpentine turns) of $15 \mu \mathrm{m}, 5 \mu \mathrm{m}$ and 1485 $\mu \mathrm{m}$, respectively (see Figure 4.18(b)). Figure 4.18(c) shows a magnified image

(a)

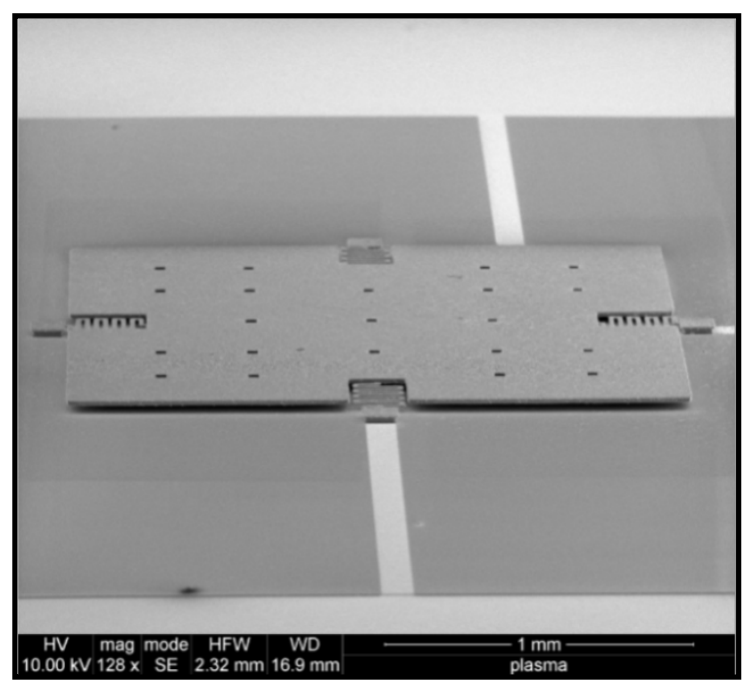

(b)

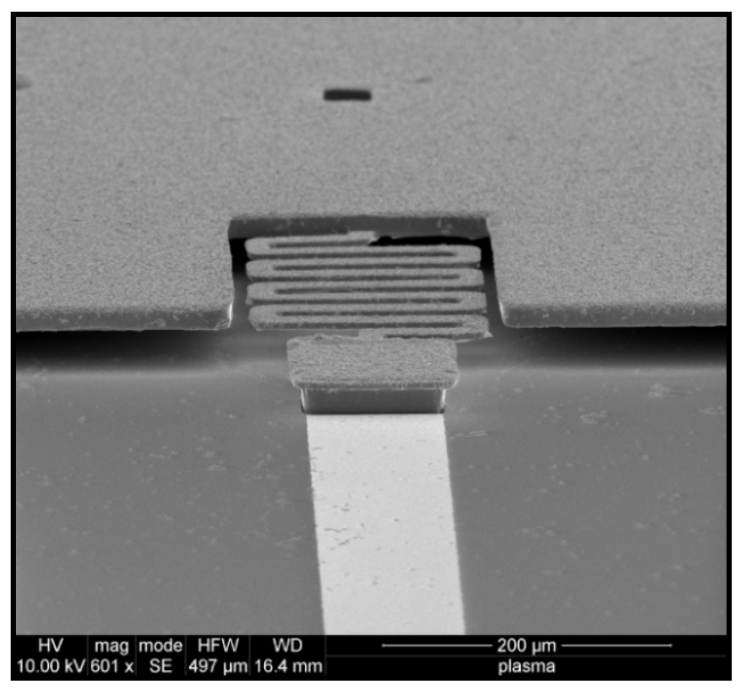


(c)

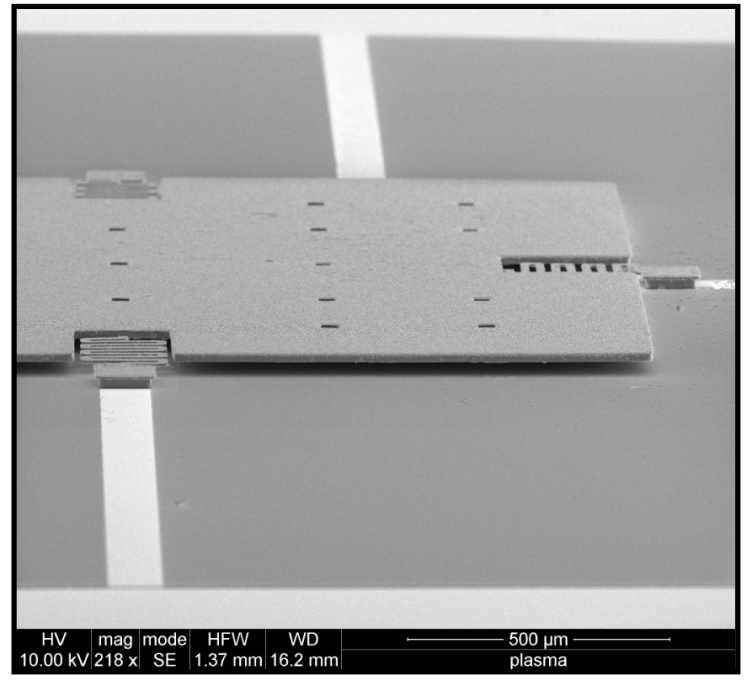

(e)

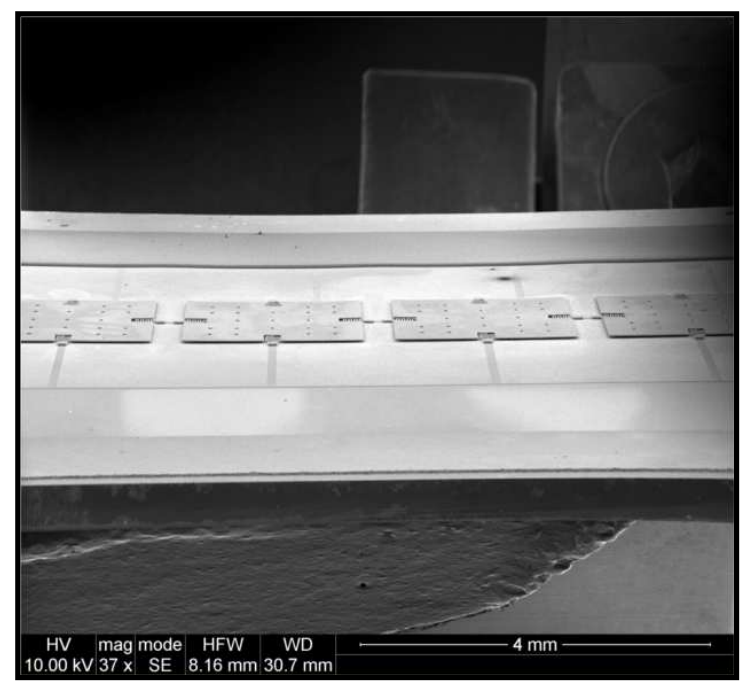

(d)

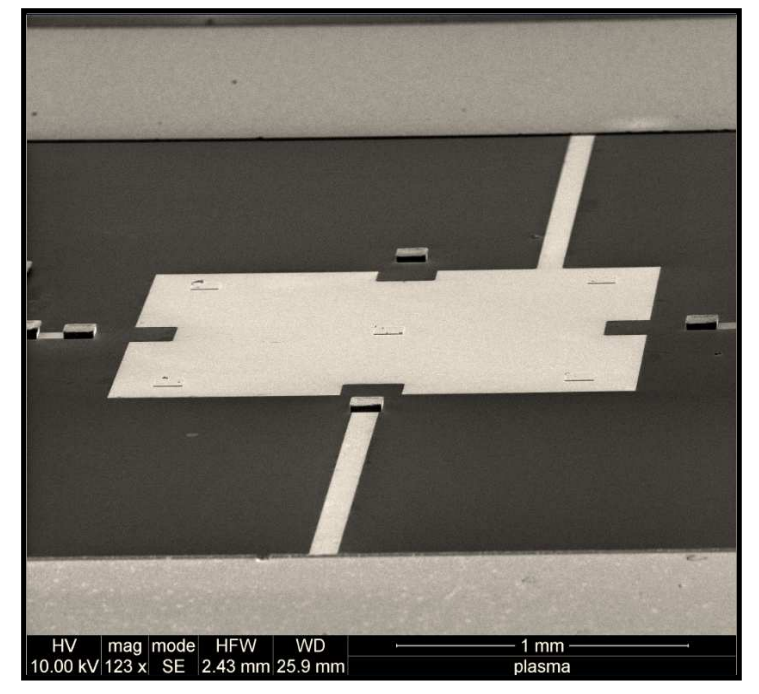

(f)

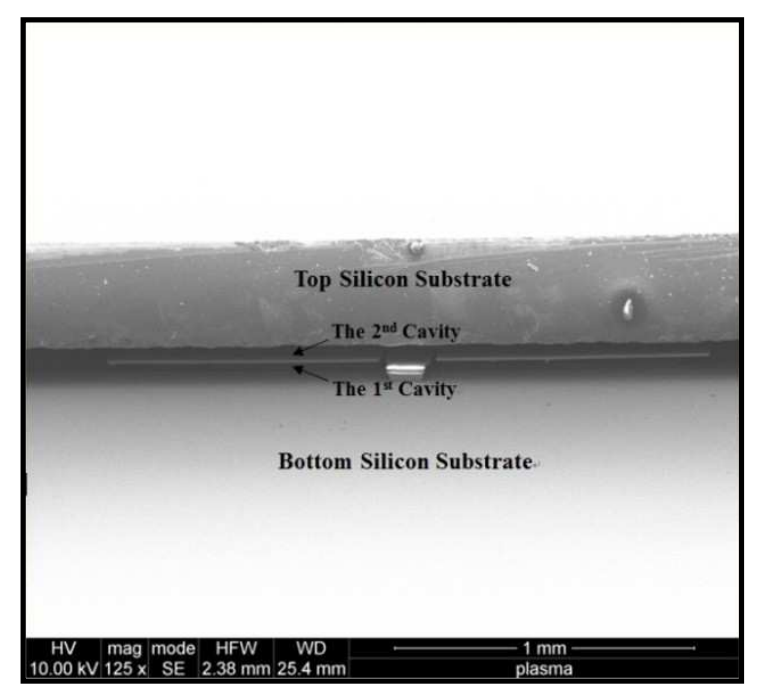

Figure 4.18. SEM micrographs of the MEMS variable capacitors with a $30 \mu \mathrm{m}$ thick movable plate and $5 \mu \mathrm{m}$ thick suspension beams before and after bonding the top cavity, (a) a $30 \mu \mathrm{m}$ thick movable plate, (b) the magnified view of a $5 \mu \mathrm{m}$ thick serpentine suspension beam, (c) the magnified view of the movable plate's edge and the $30 \mu \mathrm{m}$ air gap, (d) the $30 \mu \mathrm{m}$ height capacitor anchors and the $4 \mu \mathrm{m}$ height stoppers on the fixed plate, (e) the $1 \times 4$ capacitor array before bonding the top cavity, (f) the bonded device with two-cavity. 
of the movable plate's edge and the $30 \mu \mathrm{m}$ air gap. The SEM shows the movable plate with a flat surface and square holes for releasing the photoresist sacrificial layer. Figure 4.18(d) shows the electroplated anchors with a thickness of $30 \mu \mathrm{m}$ and the stoppers with a thickness of $4 \mu \mathrm{m}$ on a fixed plate. Figure $4.18(\mathrm{e})$ shows the $1 \times 4$ capacitor array before bonding the top cavity. The final device with two capacitive cavities is shown in Figure 4.18(f) in which the air gap height of the top and bottom capacitive cavity is both $30 \mu \mathrm{m}$.

4.2.3 Fabrication of the device with a movable plate and suspension beams thickness of $40 \mu \mathrm{m}$ and $3 \mu \mathrm{m}$

The fabrication of the MEMS variable capacitor with a $40 \mu \mathrm{m}$ thick movable plate and $3 \mu \mathrm{m}$ thick suspension beams are achieved using the same surface micromachining technologies described in the last section. However, the processes in step (6) and step (7) of the last section are modified to create the device with a $40 \mu \mathrm{m}$ thick movable plate and $3 \mu \mathrm{m}$ thick suspension beams. In the step (6), the movable plate with serpentine suspension beams is created by electroplating Ni for $3 \mu \mathrm{m}$ (instead of $5 \mu \mathrm{m}$ ) inside of a $10 \mu \mathrm{m}$ AZ-P4620 photoresist mold. In the step (7), an AZ-P4620 photoresist layer with a thickness of $30 \mu \mathrm{m}$ (instead of $20 \mu \mathrm{m}$ ) is added on the previous $10 \mu \mathrm{m} \mathrm{AZ-P4620}$ photoresist mold made in the step (6), in order to increase the total thickness of the movable plate up to $40 \mu \mathrm{m}$ (instead of $30 \mu \mathrm{m}$ ) by Ni electroplating.

The MEMS variable capacitors with a $40 \mu \mathrm{m}$ thick movable plate and $3 \mu \mathrm{m}$ thick suspension beams have been successfully fabricated. The SEM micrographs of the device with a $40 \mu \mathrm{m}$ thick movable plate and $3 \mu \mathrm{m}$ thick suspension beams are shown in Figure 4.19. The movable plate with an area of $2 \times 2 \mathrm{~mm}^{2}$ and a thickness of $40 \mu \mathrm{m}$ is well 
(a)

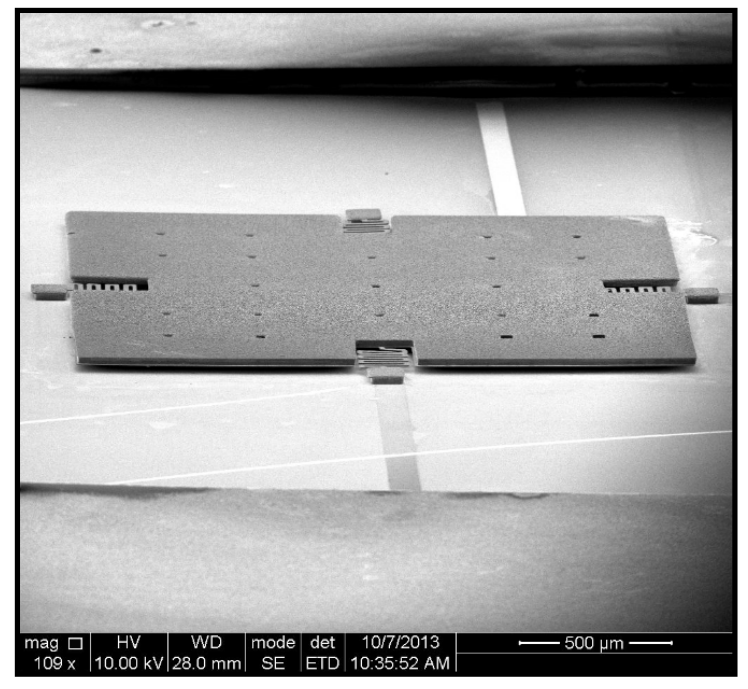

(c)

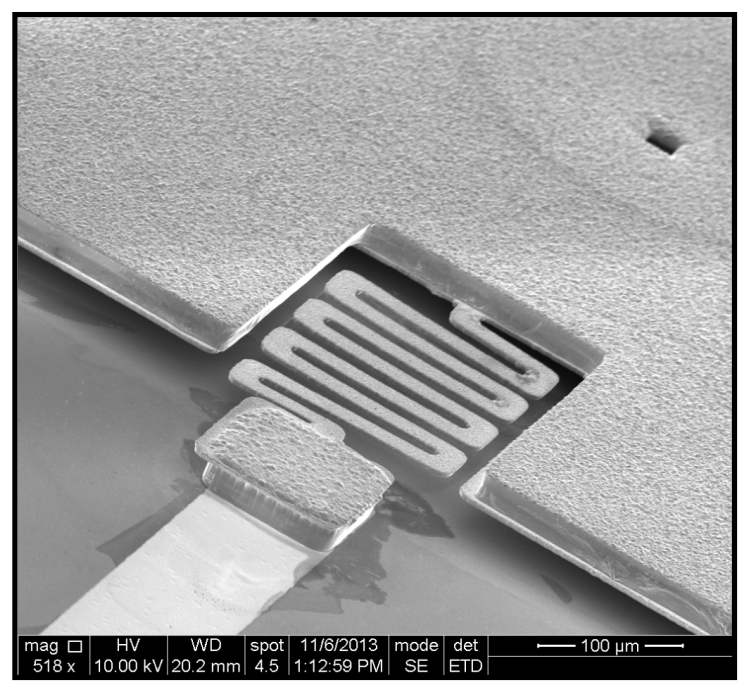

(b)

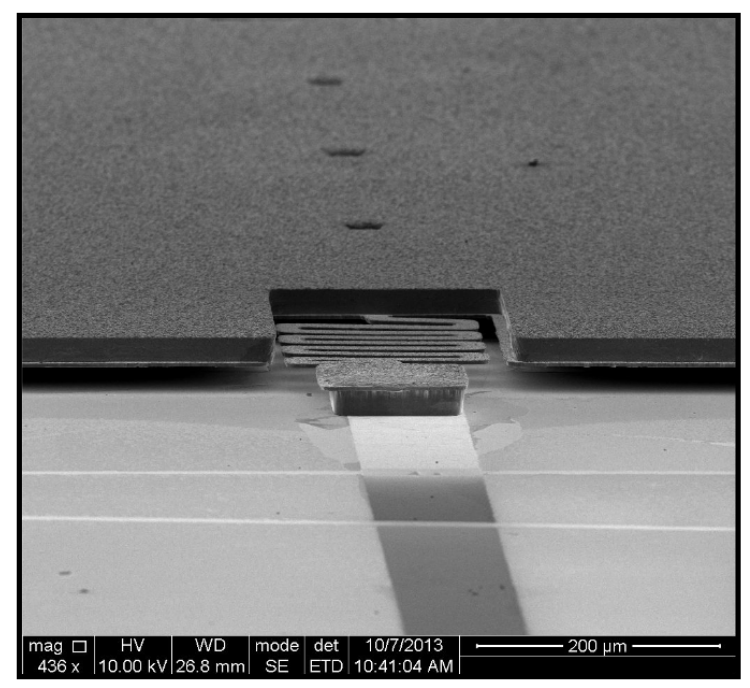

(d)

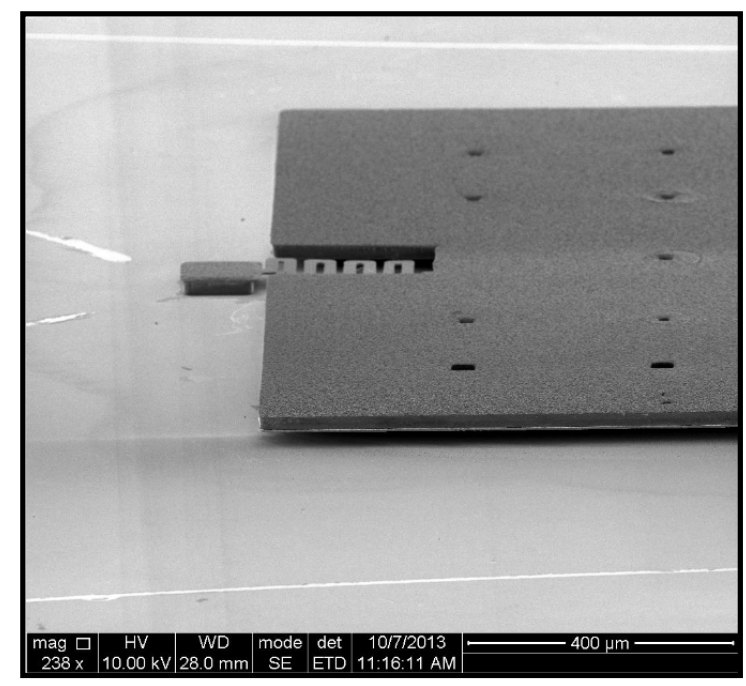

Figure 4.19. SEM micrographs of the MEMS variable capacitors with a $40 \mu \mathrm{m}$ thick movable plate and $3 \mu \mathrm{m}$ thick suspension beams before and after bonding the top cavity. (a) a $40 \mu \mathrm{m}$ thick movable plate, (b) the magnified view of $3 \mu \mathrm{m}$ thick serpentine suspension beam and $30 \mu \mathrm{m}$ air gap, (c) a magnified view of $3 \mu \mathrm{m}$ thick serpentine suspension beam captured from a different angle, (d) the movable plate's edge and $30 \mu \mathrm{m}$ air gap. 
suspended by the $3 \mu \mathrm{m}$ thick suspension beams and $30 \mu \mathrm{m}$ height anchors as shown in Figure 4.19(a). The serpentine suspension beam made with a width, thickness and total length (four serpentine turns) of $15 \mu \mathrm{m}, 3 \mu \mathrm{m}$ and $1485 \mu \mathrm{m}$, respectively, is shown in Figure 4.19(b) and 4.19(c) which were captured from different angles. Figure 4.19(d) is a magnified image on the plate's edge and $30 \mu \mathrm{m}$ air gap. It is demonstrated in the SEMs that the movable plate has a flat surface and clear suspension. The table 9 summarizes the dimensions of all fabricated devices demonstrated in the section 4.2.2 and 4.2.3.

Table 9. Summary of the dimensions of all fabricated devices.

\begin{tabular}{|c|c|c|c|c|}
\hline & $\begin{array}{r}\text { Fabrica } \\
\text { both } 4\end{array}$ & $\begin{array}{l}\text { device in } \\
\text { nd } 4.2 .3\end{array}$ & $\begin{array}{c}\text { Fabricated } \\
\text { devices in 4.2.2 }\end{array}$ & $\begin{array}{c}\text { Fabricated } \\
\text { devices in 4.2.3 }\end{array}$ \\
\hline & $\begin{array}{c}\text { Width } \\
(\mu \mathrm{m})\end{array}$ & $\begin{array}{c}\text { Length } \\
(\mu \mathrm{m})\end{array}$ & $\begin{array}{c}\text { Thickness/Height } \\
(\mu \mathrm{m})\end{array}$ & $\begin{array}{l}\text { Thickness/Height } \\
\qquad(\mu \mathrm{m})\end{array}$ \\
\hline $\begin{array}{l}\text { Movable } \\
\text { plate }\end{array}$ & 2000 & 2000 & 30 & 40 \\
\hline $\begin{array}{l}\text { Top and } \\
\text { bottom fixed } \\
\text { plates }\end{array}$ & 2000 & 2000 & 0.25 & 0.25 \\
\hline $\begin{array}{l}\text { Serpentine } \\
\text { suspension } \\
\text { beams }\end{array}$ & 15 & 1485 & 5 & 3 \\
\hline Anchors & 100 & 100 & 30 & 30 \\
\hline Stopper & 100 & 100 & 4 & 2 \\
\hline Bonding tabs & 2000 & $\begin{array}{c}3500 \text { and } \\
11340\end{array}$ & 30 & 30 \\
\hline Air gaps & & & 30 & 30 \\
\hline
\end{tabular}




\section{CHAPTER 5}

\section{RESULTS AND DISCUSSION}

This chapter presents the experimental characterization setup and detailed characterization of the fabricated single MEMS variable capacitor and $1 \times 4$ capacitor array. The experimental results, including resonance frequency, RMS output voltage and power across the load resistors, resonance frequency shifting, under a variety of vibration conditions will be discussed.

\subsection{Experimental setup}

The characterization strategy has been introduced in the section 2.3.3 of Chapter 2 as follows: The top and bottom capacitive cavities are initially charged by a low DC voltage source. When the plate moves down, capacitance of the bottom cavity increases and takes in charge from the low DC power source. Charging of bottom cavity stops when the plate reaches the lowest bottom position, then the plate tries to bounce back up so the gap of the bottom cavity increases and the gap of the top cavity decreases. Meanwhile, capacitance of the bottom cavity decreases and releases charge to corresponding load resistor, and capacitance of the top cavity starts increasing thereby taking charge from the low DC power source. Discharging of top cavity to its load resistor starts as its capacitance decreases when plate reaches the highest top position and then moves back down again. Consequently, two out-of-phase AC signals are generated from two capacitive cavities as the above sequence repeats when the movable plate is 
vibrating. To realize this charging method, an electrical characterization circuit has been developed, and is shown in Figure 5.1.

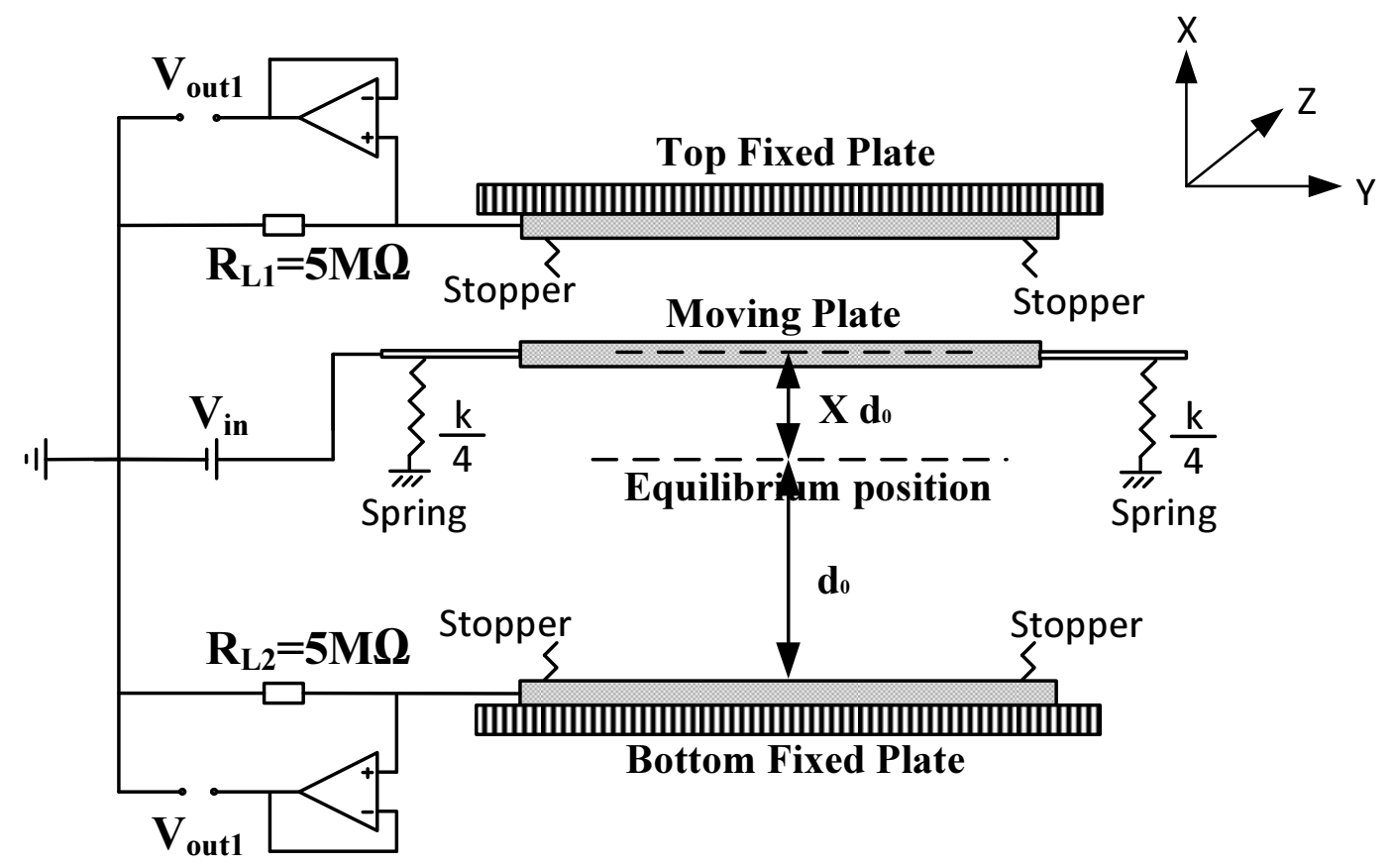

Figure 5.1. The schematic of the electrical characterization circuit.

The MEMS variable capacitor energy harvester was experimentally characterized using a printed circuit board test stage mounted on a shaker (Model Shop, TMS, K2007E type). The experimental setup is shown in Figure 5.2. The device was firmly attached on the test stage. An analog accelerometer (Silicon Designs 1210L-050 type) was also mounted on the test stage beside the device and was used to measure the excitation amplitude with respect to gravity (g, acceleration). For an electrostatic energy harvester without built-in potential by an electret layer, it requires an external DC bias to operate and generate the energy. To obtain clear signals with minimum charge loss, the experimental circuit uses high value load resistors of $5 \mathrm{M} \Omega$ to pull up the voltage signals. The voltage signals were then measured via two unity amplifiers to eliminate the current 


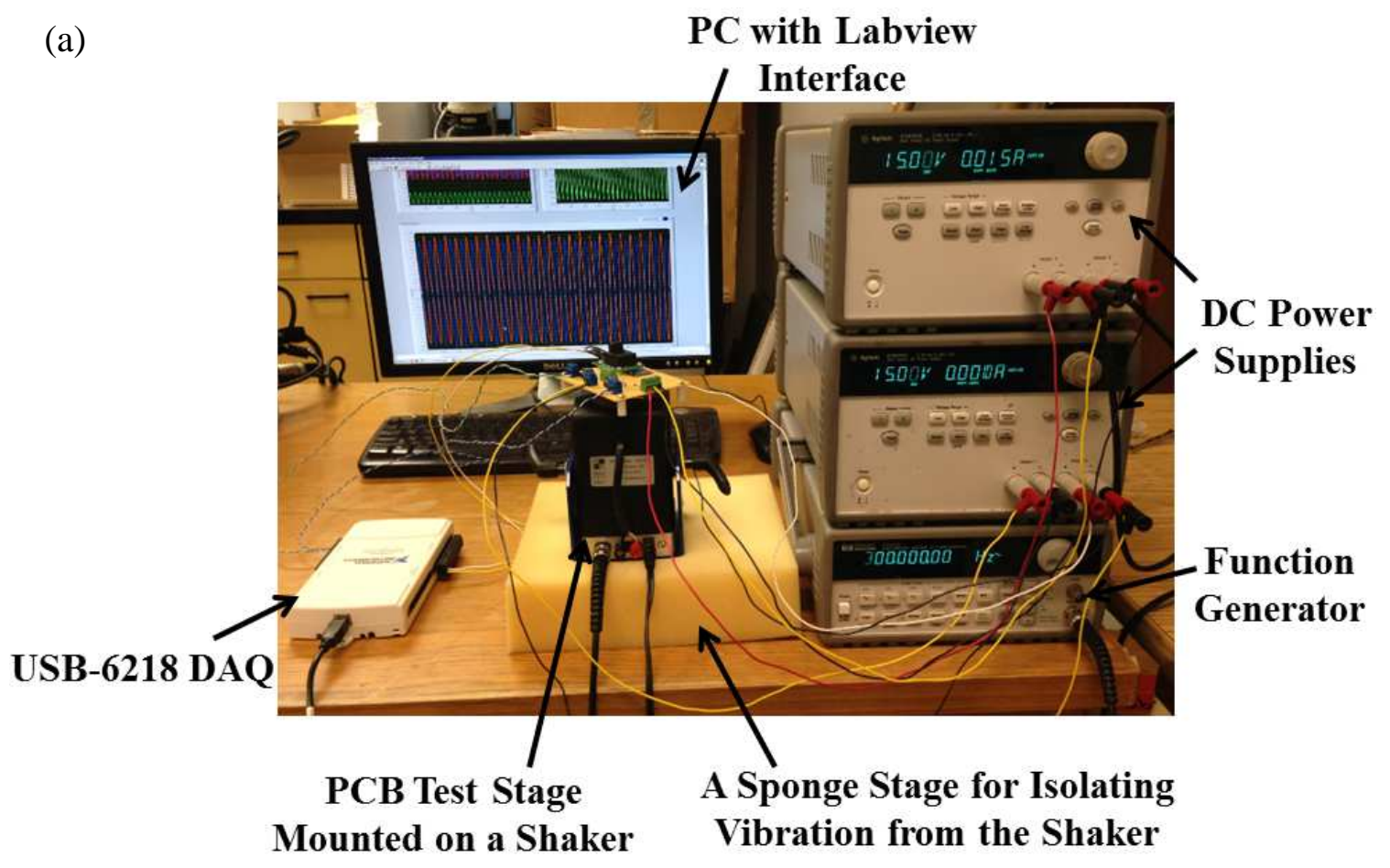

(b)

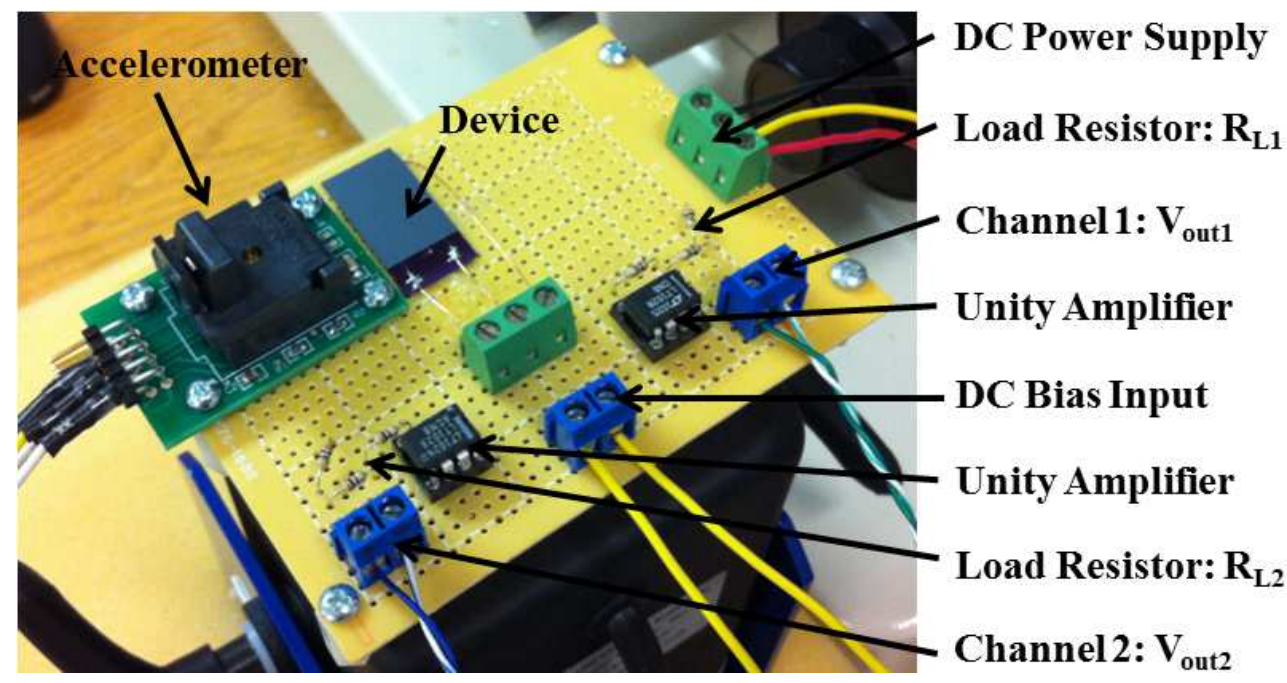

Figure 5.2. The experimental testing setup, (a) an overview of testing setup and environment, (b) the PCB test stage mounted on the shaker.

drainage when the charge flows. Therefore, when the device was being DC biased with a low DC power supply and driven into vibration by the shaker, two AC signals were generated across the two $5 \mathrm{M} \Omega$ load resistors and measured through the two unity amplifiers using a National Instrument DAQ (USB-6218 DAQ) and LabView interface in 
a PC. An overview of the testing setup and environment is shown in Figure 5.2(a). The two DC power supplies were used to provide the DC bias to the device and to power the unity amplifiers. The function generator was used to drive the shaker into desired vibration frequency and amplitude.

A programed circuit in Labview automatically collected the data from the outputs of the unity amplifiers and the accelerometer. Each set of measurement lasted 30 seconds, during which each output generated 375 sample data. The RMS voltage across each load resistor under different vibrations (with different frequencies and accelerations) was calculated by averaging the 375 sample data from each set of measurement. The excitation acceleration (g) was monitored and calibrated using output date from the accelerometer. Both single capacitor and $1 \times 4$ capacitor array have been tested using the setup described above.

\subsection{Frequency sweep testing at fixed excitation amplitude}

5.2.1 Power harvesting devices with a movable plate and suspension beams thickness of $30 \mu \mathrm{m}$ and $5 \mu \mathrm{m}$

Figure 5.3 shows two time domain plots of AC output voltage across the load resistors when the single capacitor and capacitor array with a $30 \mu \mathrm{m}$ thick movable plate and $5 \mu \mathrm{m}$ thick suspension beams were being excited. In the Figure, two AC signals (marked as blue and red curves) corresponding to the outputs from the top and bottom capacitive cavities, respectively, were always out-of-phase.

The devices were characterized by sweeping the excitation frequency from 300 to $700 \mathrm{~Hz}$ with steps of $10 \mathrm{~Hz}$ under sinusoidal excitations with constant acceleration. The RMS output voltage under DC bias of $6 \mathrm{~V}, 9 \mathrm{~V}, 12 \mathrm{~V}$ and $15 \mathrm{~V}$ from the bottom cavity 
(a)

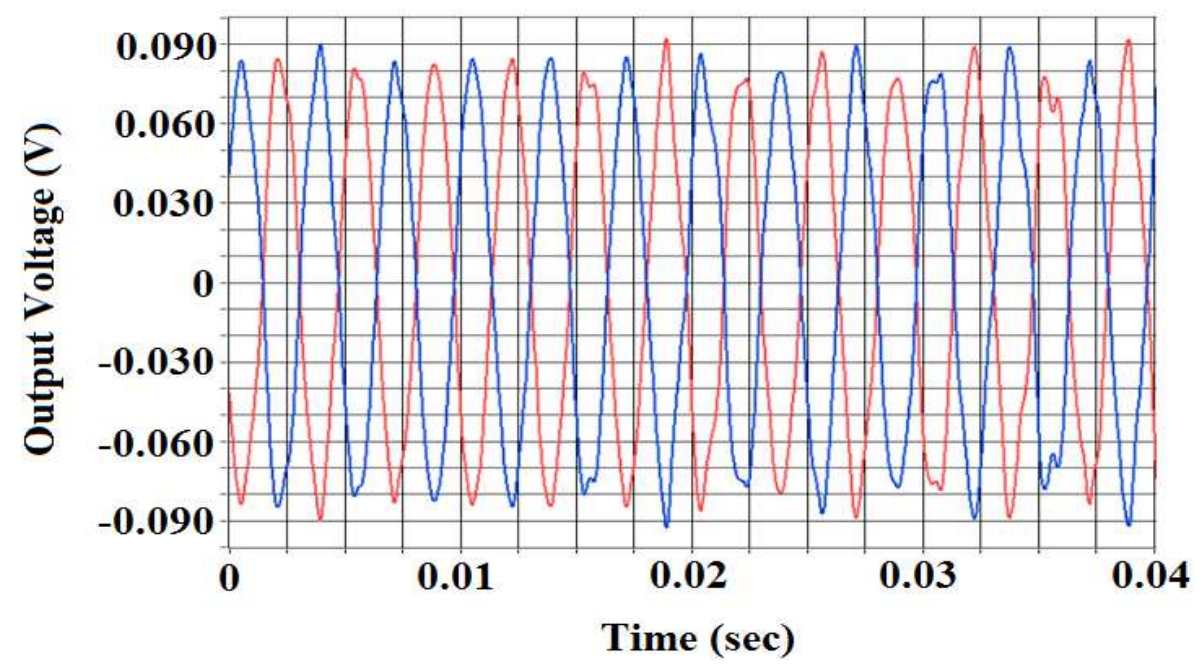

(b)

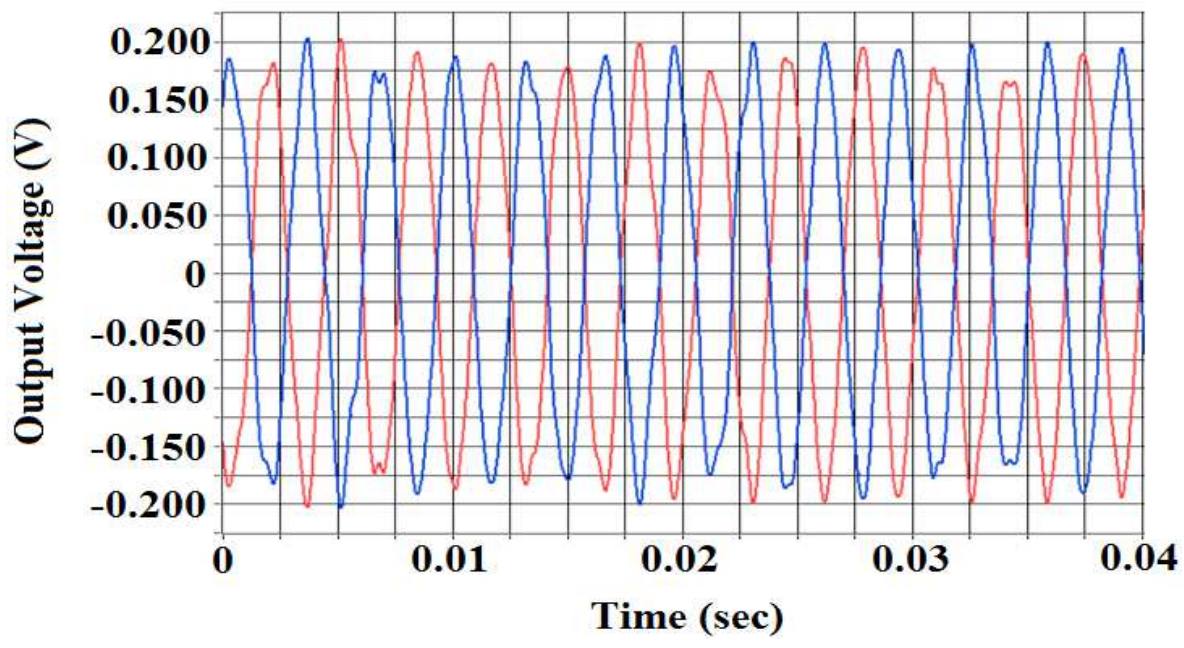

Figure 5.3. Time domain plots of AC output voltage across the load resistors from the devices with a movable plate and suspension beams thickness of $30 \mu \mathrm{m}$ and $5 \mu \mathrm{m}$, respectively, (a) single capacitor device under $15 \mathrm{~V}$ DC bias, $300 \mathrm{~Hz}$ and $1.026 \mathrm{~g}$ excitation, (b) $1 \times 4$ capacitor array device under $15 \mathrm{~V} \mathrm{DC}$ bias, $300 \mathrm{~Hz}$ and $1.020 \mathrm{~g}$ excitation.

$\left(\mathrm{V}_{\text {out2 }}\right)$ of a single capacitor and $1 \times 4$ capacitor array were plotted as function of frequency in Figure 5.4(a) and 5.4(b), respectively. The resonance frequency was observed around $500 \mathrm{~Hz}$ for both single capacitor and capacitor array devices. However, the device of capacitor array has broader bandwidth of resonance around 480 to $520 \mathrm{~Hz}$ 
(a)

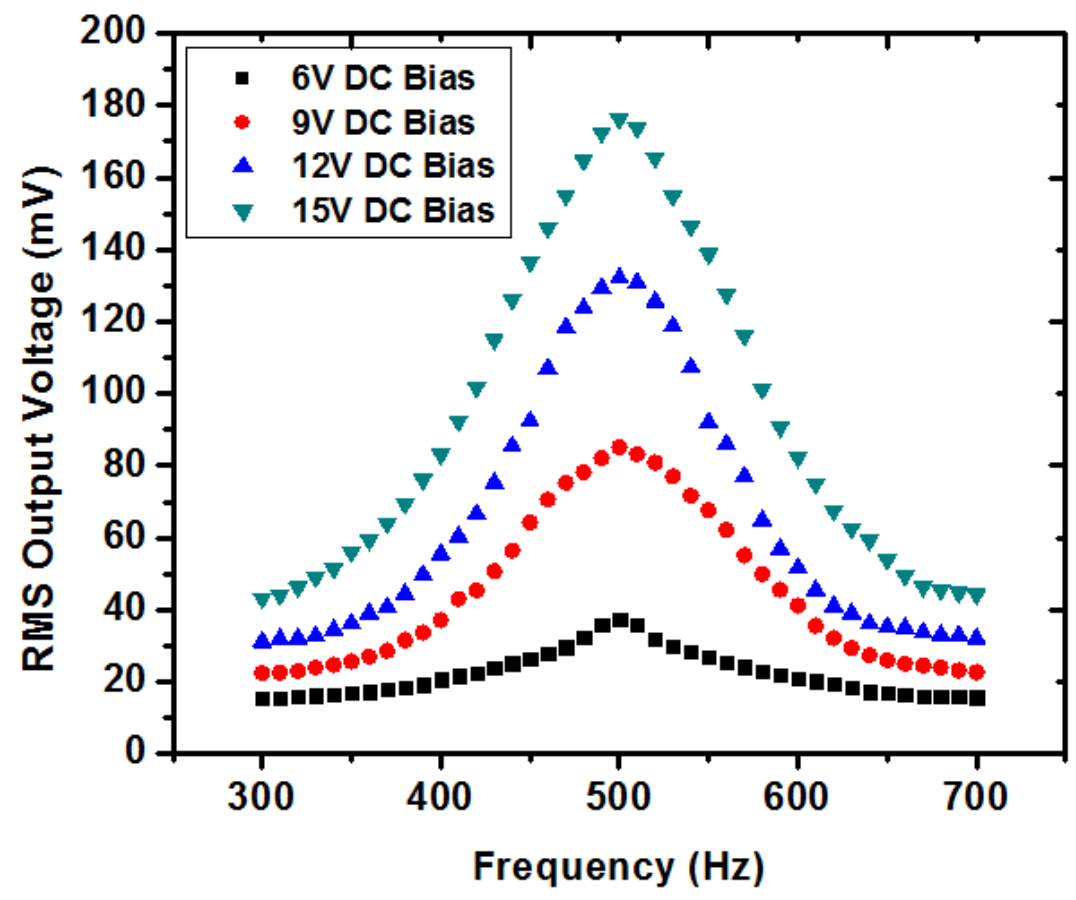

(b)

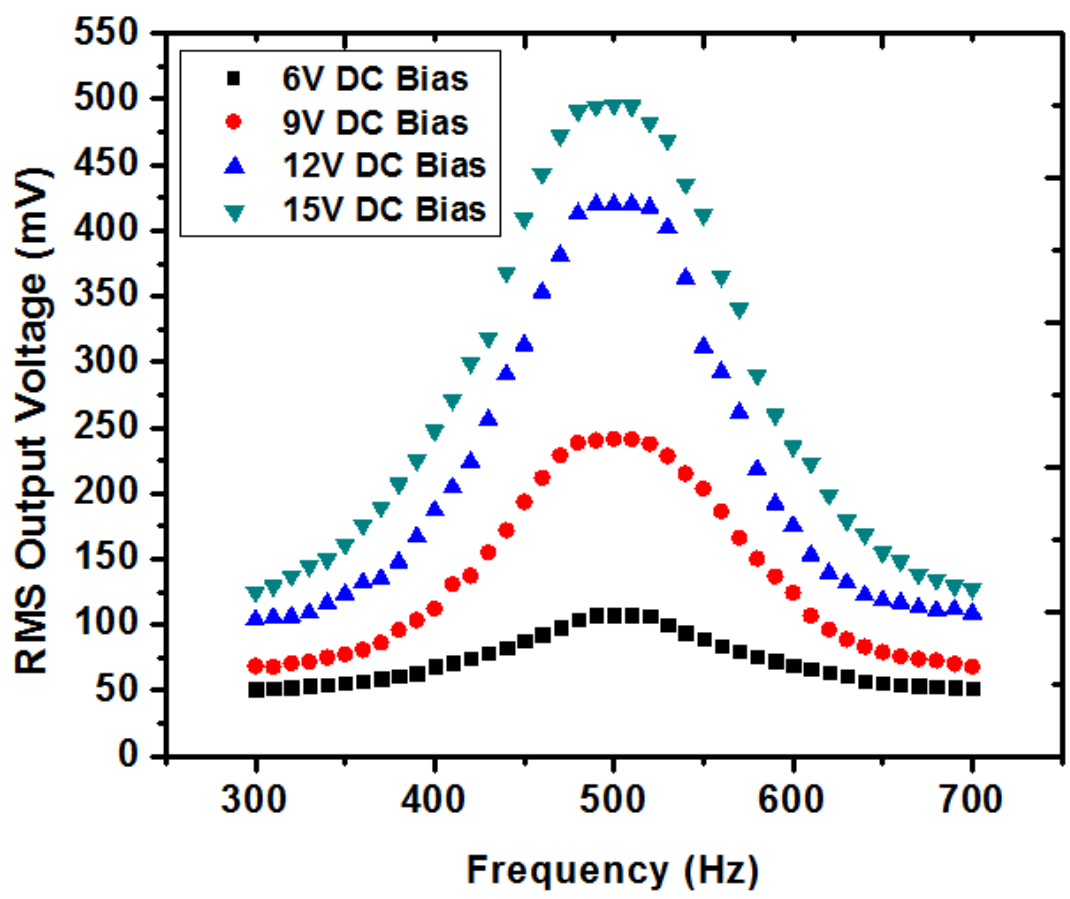

Figure 5.4. The RMS output voltage from bottom cavity of the devices with a movable plate and suspension beams thickness of $30 \mu \mathrm{m}$ and $5 \mu \mathrm{m}$, respectively, as frequency sweeps, (a) a single capacitor under RMS excitation of $1.026 \mathrm{~g}$, (b) $1 \times 4$ capacitor array under RMS excitation of $1.020 \mathrm{~g}$. 
(a)

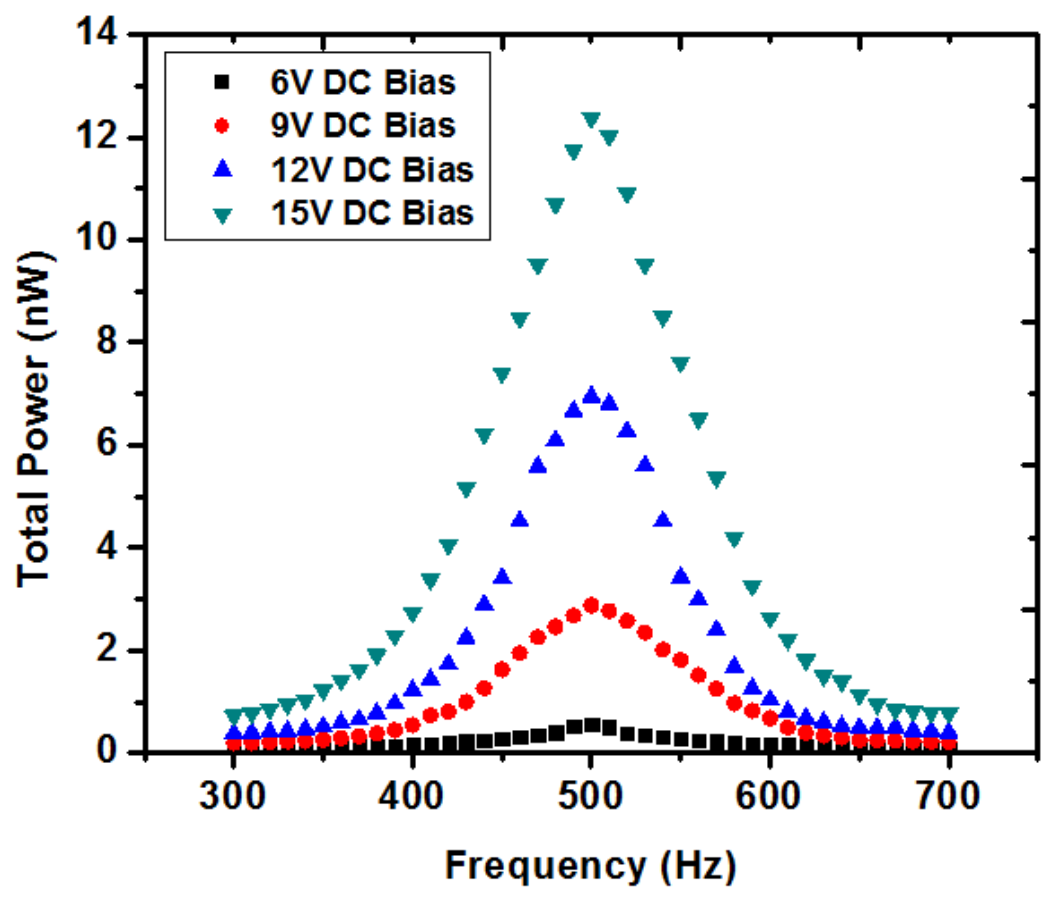

(b)

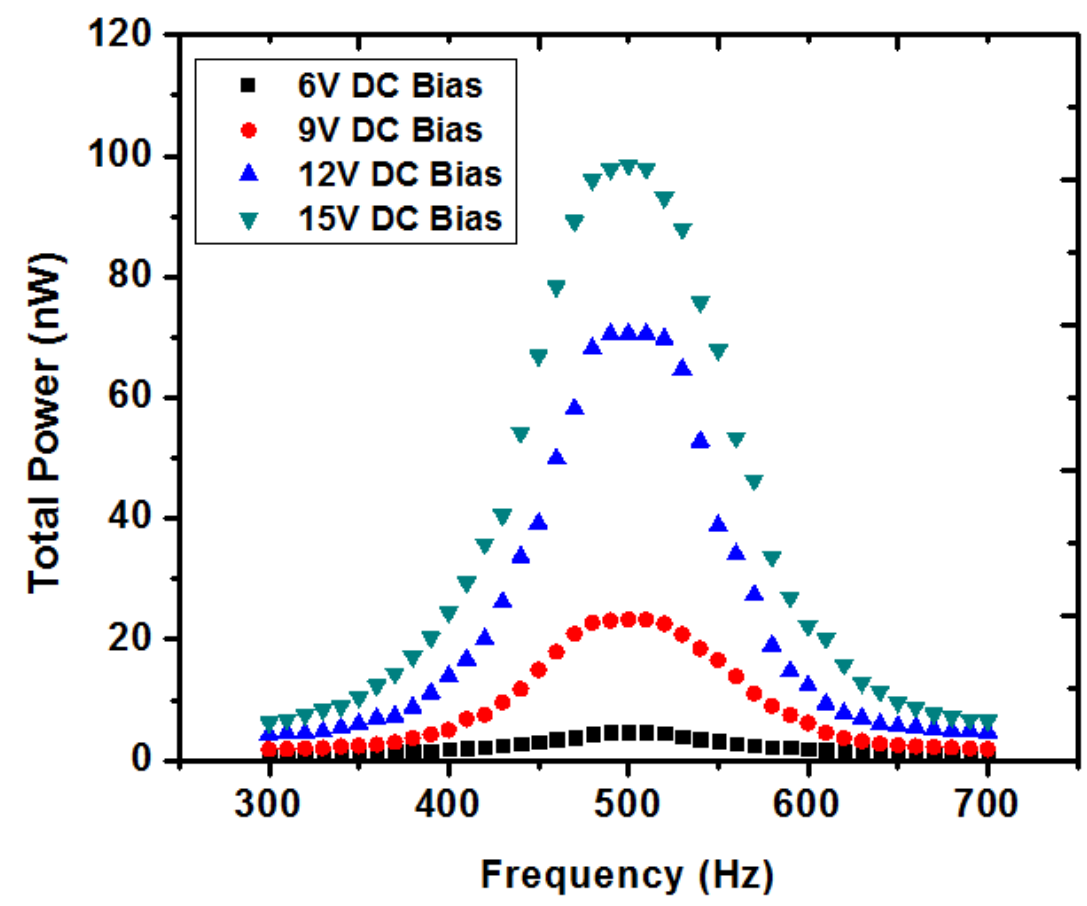

Figure 5.5. The total power across the load resistors from the devices with a movable plate and suspension beams thickness of $30 \mu \mathrm{m}$ and $5 \mu \mathrm{m}$, respectively, as frequency sweeps, (a) a single capacitor under RMS excitation of $1.026 \mathrm{~g}$, (b) $1 \times 4$ capacitor array under RMS excitation of $1.020 \mathrm{~g}$. 
than the device of single capacitor that has a peak at $500 \mathrm{~Hz}$, which is ascribed to slightly different resonance responses from each capacitor. We have selected the output voltage from bottom cavity $\left(\mathrm{V}_{\text {out2 }}\right)$ to show the devices' responses because the experimental results show that the outputs from top $\left(\mathrm{V}_{\text {out } 1}\right)$ and bottom $\left(\mathrm{V}_{\text {out2 }}\right)$ cavity are nearly equivalent.

In addition, because a clear resonant behavior was observed, we believe that the experimental forcing amplitude has not yet reached the value that we studied in the simulations and no contact between the movable plate and the fixed plates has taken place. The total power $\left(\mathrm{P}=\mathrm{V}_{\text {out }}{ }^{2} / \mathrm{R}\right)$ across the load resistors from the device of single capacitor and the device of capacitor array were calculated and plotted as function of frequency in Figure 5.5(a) and 5.5(b), respectively.

5.2.2 Power harvesting devices with a movable plate and suspension beams thickness of $40 \mu \mathrm{m}$ and $3 \mu \mathrm{m}$

The single capacitor and capacitor array with a $40 \mu \mathrm{m}$ thick movable plate and 3 $\mu \mathrm{m}$ thick suspension beams were similarly characterized as described in section 5.2.1 by sweeping the excitation frequency from 100 to $600 \mathrm{~Hz}$ with steps of $10 \mathrm{~Hz}$ under sinusoidal excitations with constant acceleration. The RMS output voltage under bias DC of $6 \mathrm{~V}, 9 \mathrm{~V}, 12 \mathrm{~V}$ and $15 \mathrm{~V}$ from bottom cavity $\left(\mathrm{V}_{\text {out2 }}\right)$ of each device were plotted as function of frequency in Figure 5.6(a) and 5.6(b). The single capacitor and capacitor array with a $40 \mu \mathrm{m}$ movable plate and $3 \mu \mathrm{m}$ suspension beams were observed with lower resonance frequency which is at around $290 \mathrm{~Hz}$. The result proves that the resonance frequency of our energy harvesters can be effectively reduced by increasing the weight of movable proof mass plate and/or by softening the suspension beams. 
(a)

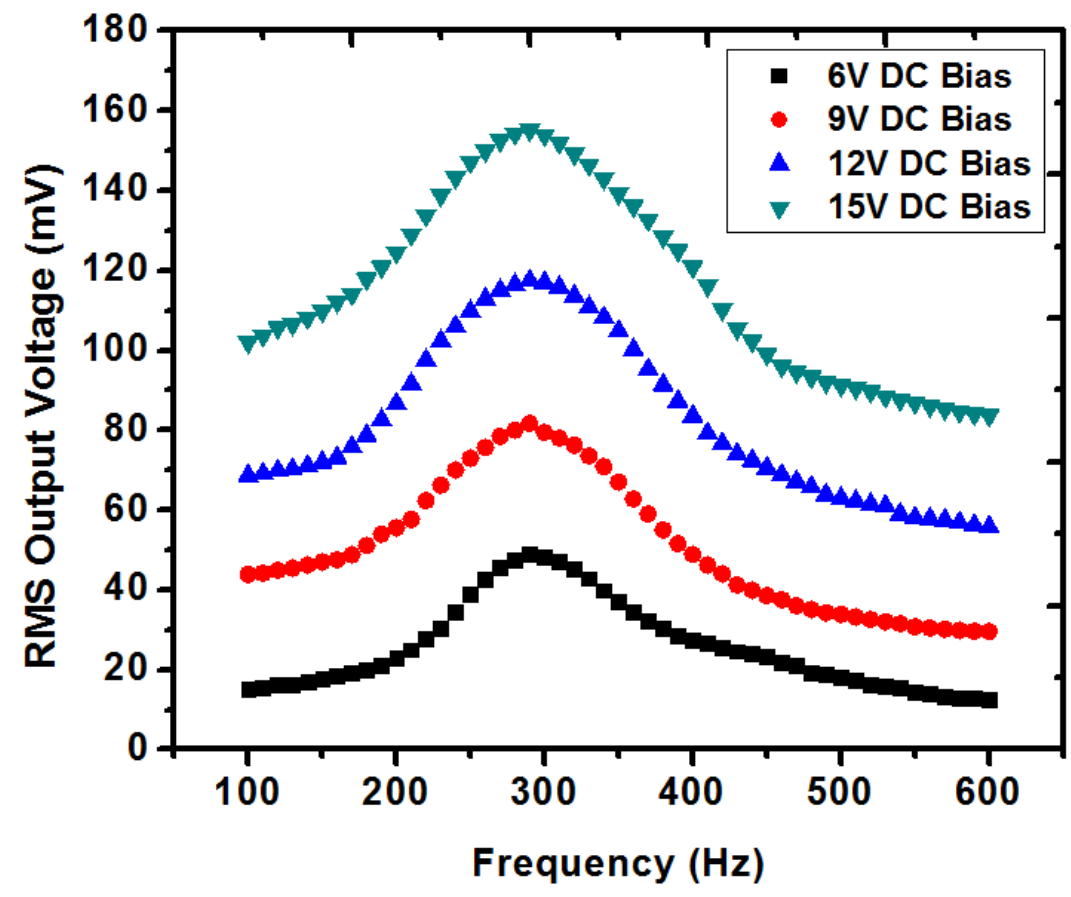

(b)

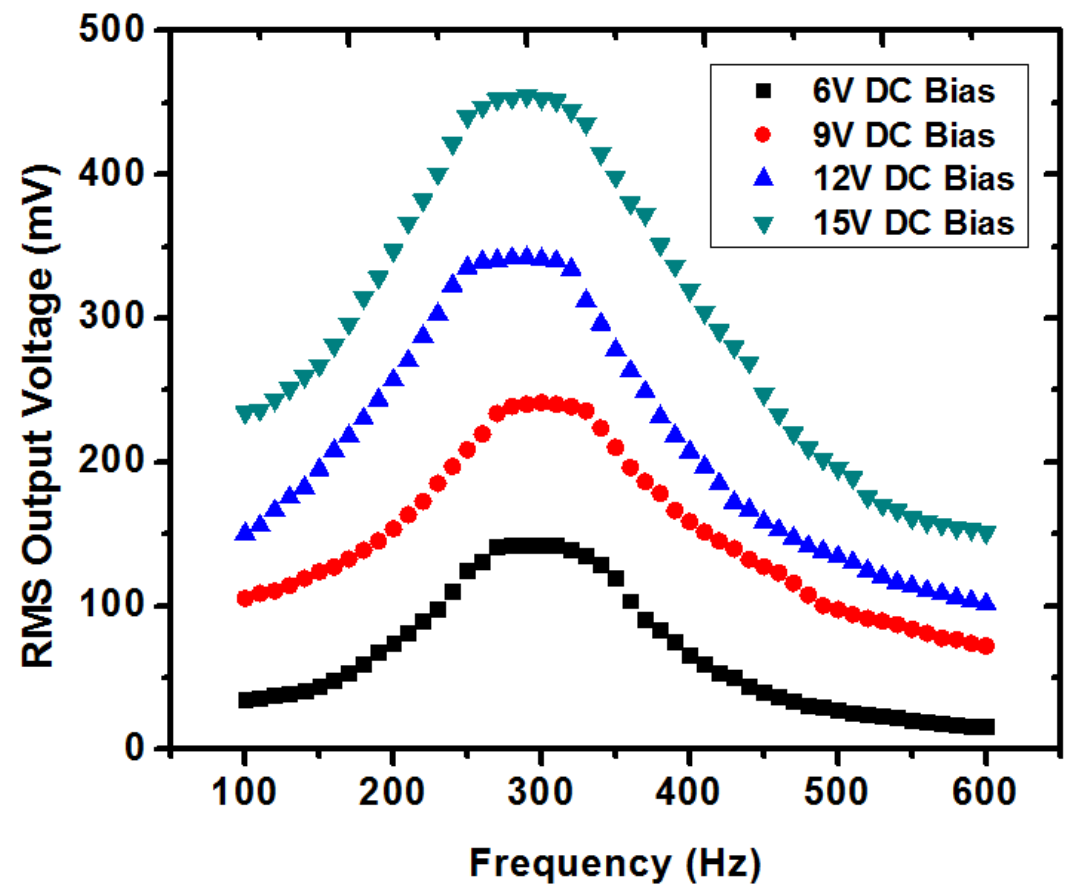

Figure 5.6. The RMS output voltage from bottom cavity of the devices with a movable plate and suspension beams thickness of $40 \mu \mathrm{m}$ and $3 \mu \mathrm{m}$, respectively, as frequency sweeps, (a) a single capacitor under RMS excitation of $1.041 \mathrm{~g}$, (b) $1 \times 4$ capacitor array under RMS excitation of $1.018 \mathrm{~g}$. 
(a)

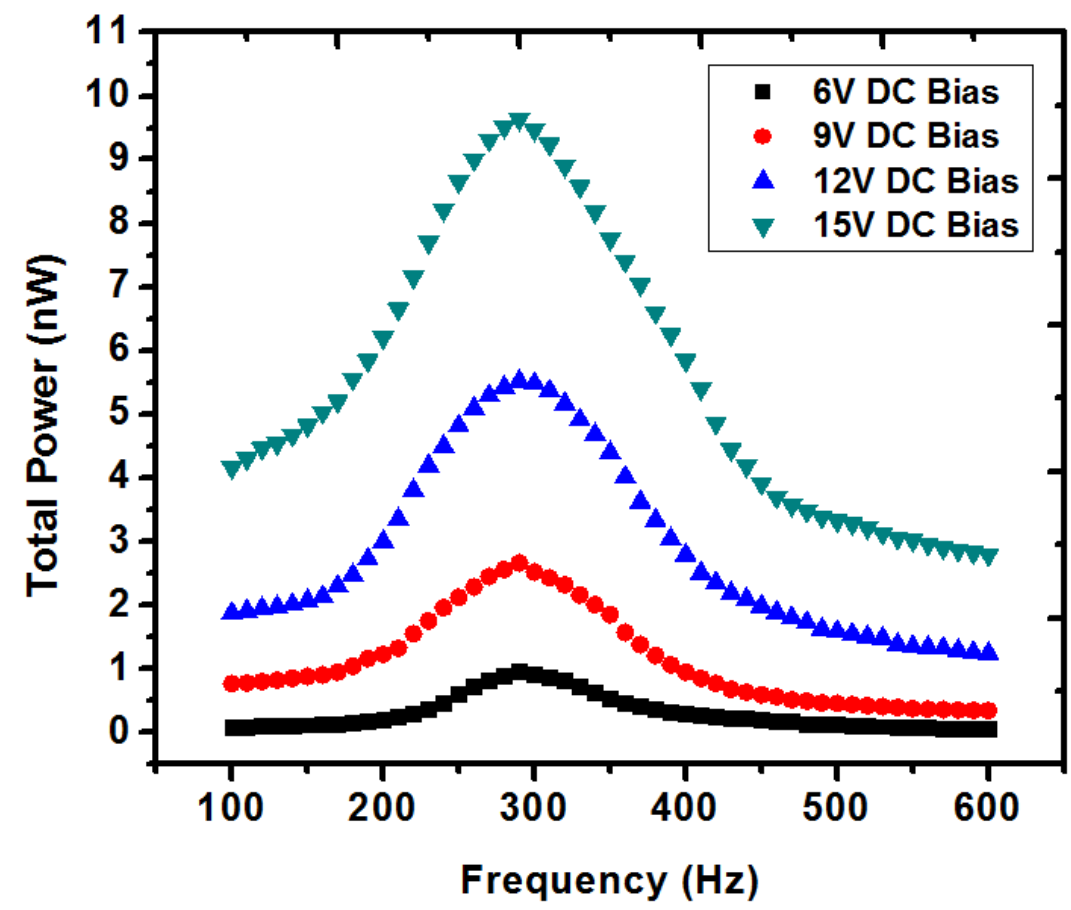

(b)

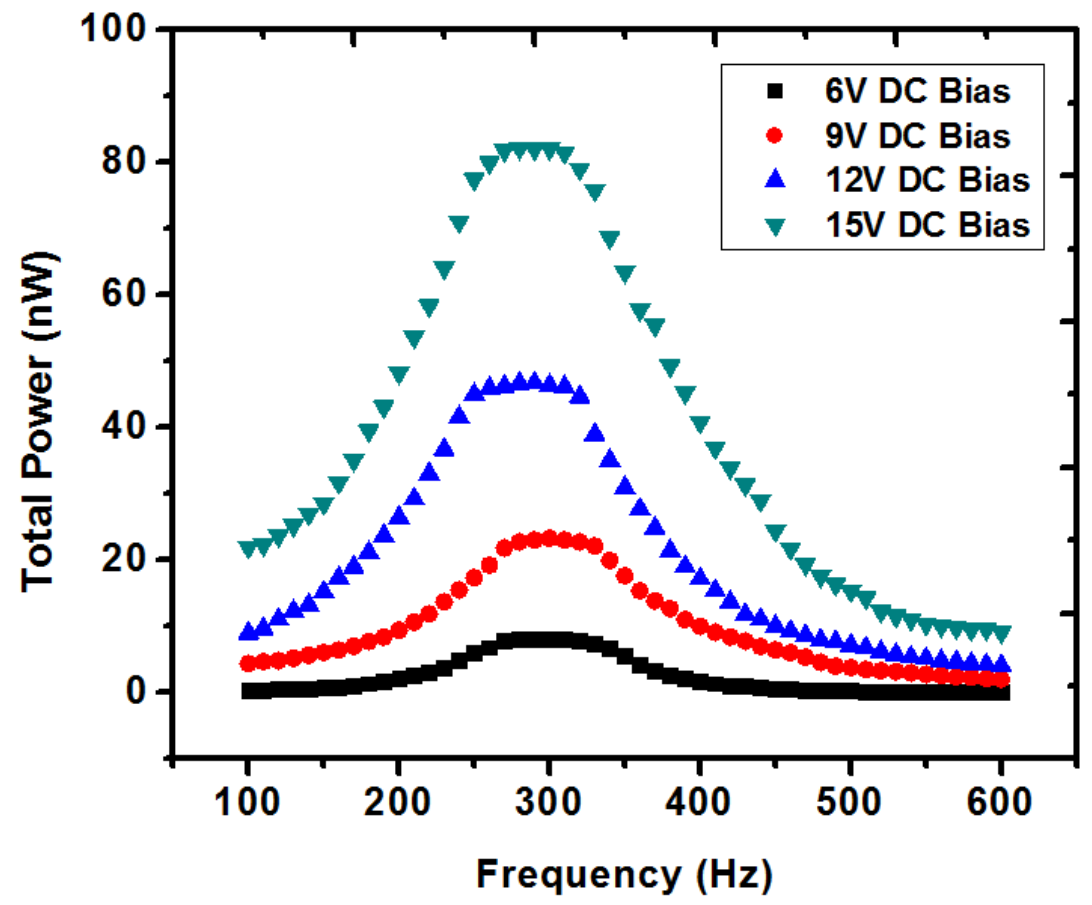

Figure 5.7. The total power across two load resistors from the devices with a movable plate and suspension beams thickness of $40 \mu \mathrm{m}$ and $3 \mu \mathrm{m}$, respectively, as frequency sweeps, (a) a single capacitor under RMS excitation of $1.041 \mathrm{~g}$, (b) $1 \times 4$ capacitor array under RMS excitation of $1.018 \mathrm{~g}$. 
Therefore, our energy harvester can be customized for the application in an environment with specific vibration frequency. The capacitor array was also observed with a broad bandwidth response between 270 to $320 \mathrm{~Hz}$, while the single capacitor device was sharply peaked at $290 \mathrm{~Hz}$. The corresponding total power $\left(\mathrm{P}=\mathrm{V}_{\text {out }}{ }^{2} / \mathrm{R}\right)$ across the load resistors from the single capacitor device and the capacitor array were calculated and plotted as function of frequency in Figure 5.7(a) and 5.7(b), respectively.

5.2.3 Comparison of the results in frequency sweep testing at fixed excitation amplitude

By increasing the thickness of movable plate from $30 \mu \mathrm{m}$ to $40 \mu \mathrm{m}$ and reducing the thickness of suspension beams from $5 \mu \mathrm{m}$ to $3 \mu \mathrm{m}$, the resonance frequency of the devices were successfully lowered from 500 to $300 \mathrm{~Hz}$ as shown in Figure 5.8. The $1 \times 4$ capacitor array was always observed with a broader bandwidth of resonance than the single capacitor. This might be due to slight different in resonance responses of each capacitor of the array (see Figure 5.8(a) and 5.8(b)). This effect can be utilized to optimize the response bandwidth of our power harvester devices.

\subsection{Excitation sweep testing at the resonance frequency}

5.3.1 Power harvesting devices with a movable plate and suspension beams thickness of $30 \mu \mathrm{m}$ and $5 \mu \mathrm{m}$

In this testing, the devices with a $30 \mu \mathrm{m}$ thick movable plate and $5 \mu \mathrm{m}$ thick suspension beams were characterized under a fixed excitation frequency of $500 \mathrm{~Hz}$ while sweeping the excitation amplitude. The RMS output voltage from bottom cavity $\left(\mathrm{V}_{\text {out2 }}\right)$ of the devices and the corresponding total power from the load resistors under different DC bias were observed increasing as excitation acceleration was sweeping up, and then 
(a)

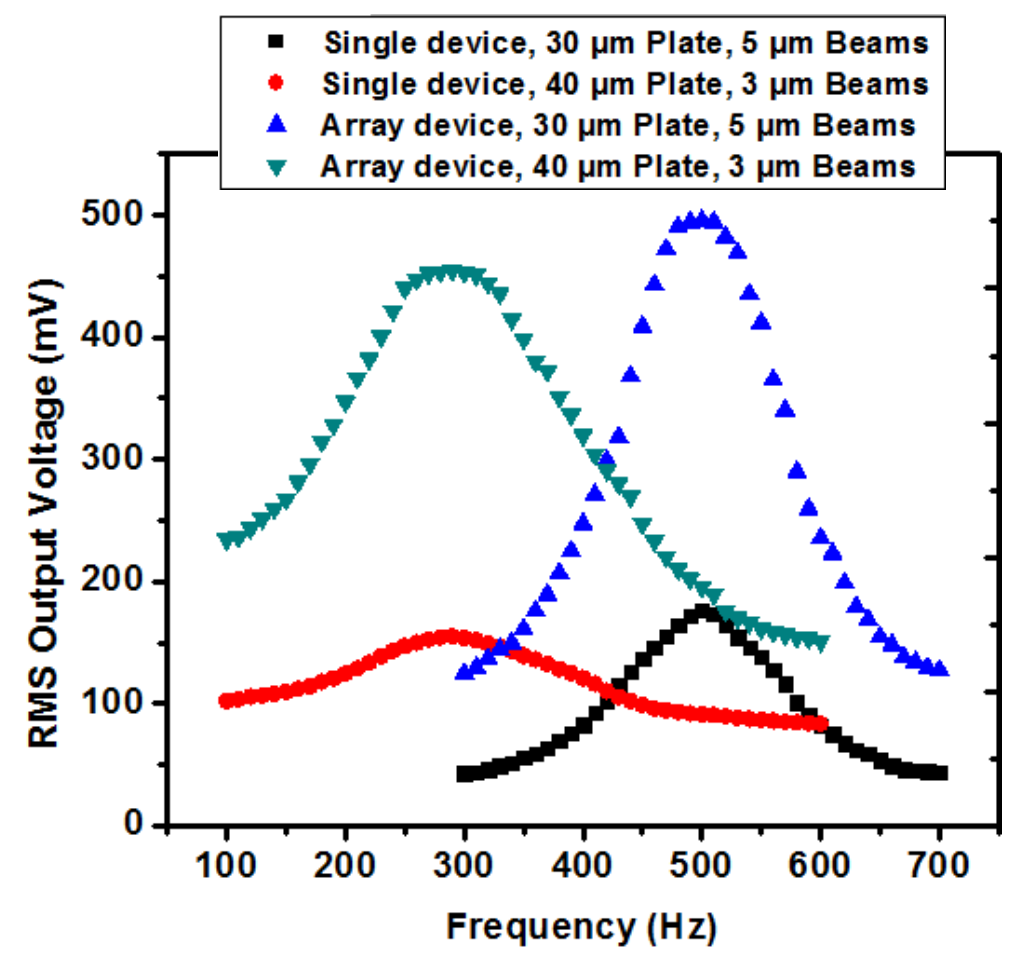

(b)

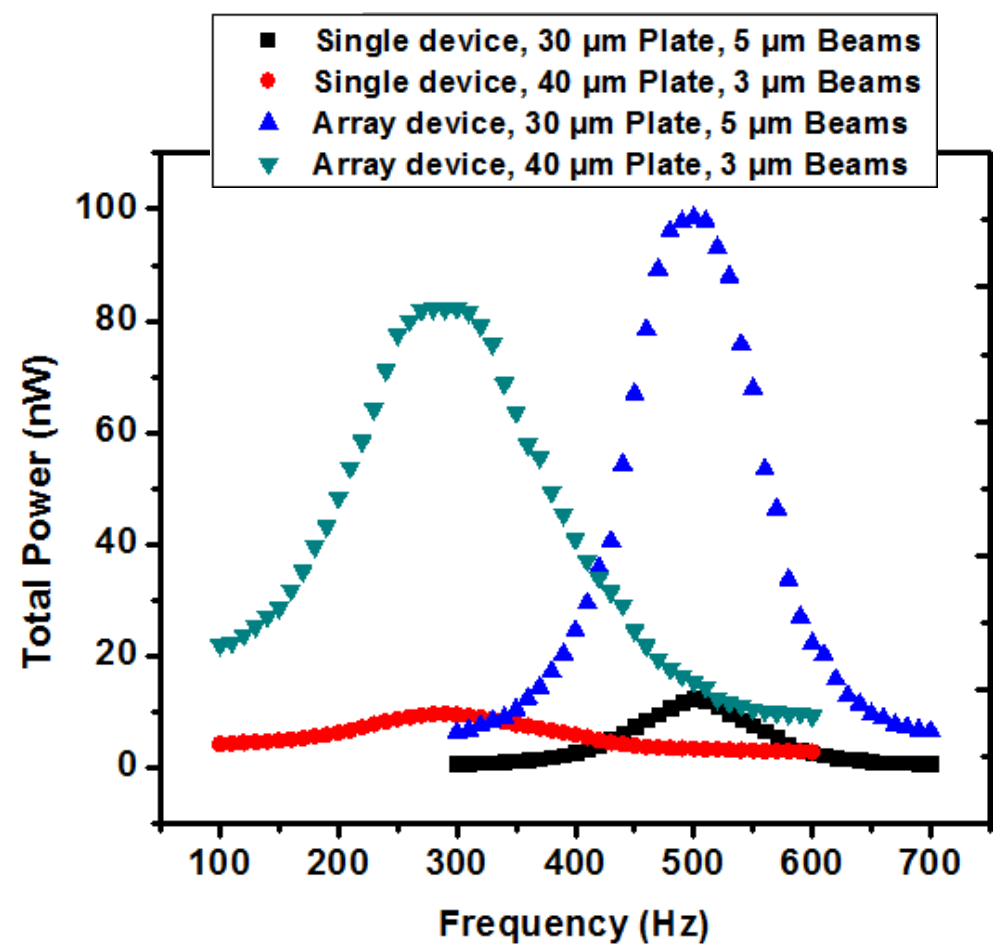

Figure 5.8. Comparisons of the results in frequency sweep testing with a DC bias of $15 \mathrm{~V}$,

(a) the RMS output voltage from bottom cavity of the devices as frequency sweeps under RMS excitation around $1 \mathrm{~g}$, (b) the total power across two load resistors as frequency sweeps under RMS excitation around $1 \mathrm{~g}$. 
finally saturating in extreme high excitation amplitude (see Figure 5.9 and Figure 5.10). The measured total power across the load resistors from single capacitor and capacitor array achieved a maximum power of $220 \mathrm{nW}$ and $2.5 \mu \mathrm{W}$, respectively, under DC bias of $15 \mathrm{~V}$ and excitation amplitude above $5 \mathrm{~g}$ as shown in Figure 5.10(a) and 5.10(b).

It is noted that the measured RMS voltage from the device capacitor array is 3.2 times (in average) higher than that of the device of single capacitor under the same excitation conditions, which indicates that the capability of charge generation from the capacitor array is 3.2 times higher in average than that of a single capacitor due to its higher variation of capacitance. Since energy harvesting from the device is directly proportional to the generation of charge flow, it can be addressed that the $1 \times 4$ capacitor array is capable of producing 11.1 times (in average) higher power than that of a single capacitor.

5.3.2 Power harvesting devices with a movable plate and suspension beams thickness of $40 \mu \mathrm{m}$ and $3 \mu \mathrm{m}$

The excitation sweep testing for the devices with a $40 \mu \mathrm{m}$ thick movable plate and $3 \mu \mathrm{m}$ thick suspension beams was similarly implemented as described in section 5.3.1. The single capacitor and capacitor array were characterized under a fixed excitation frequency of $290 \mathrm{~Hz}$ while sweeping the excitation amplitude between 0 to $7 \mathrm{~g}$. The RMS output voltage from bottom cavity $\left(\mathrm{V}_{\text {out } 2}\right)$ of the devices and the corresponding total power across the load resistors under different DC bias were observed increasing as excitation acceleration was sweeping up, and then finally saturating under high excitation acceleration above $3.7 \mathrm{~g}$ as shown in Figure 5.11 and Figure 5.12. The measured total power across the load resistors from the device of single capacitor and the device of 
(a)

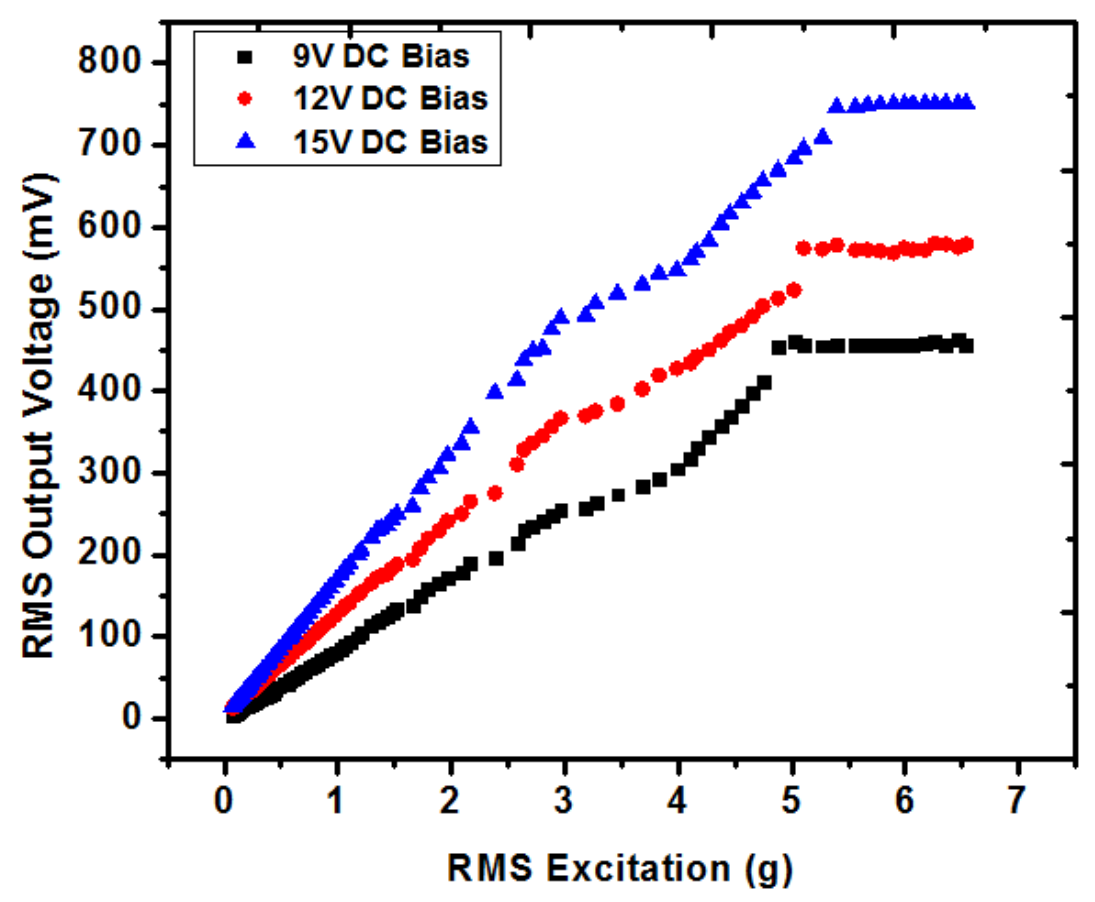

(b)

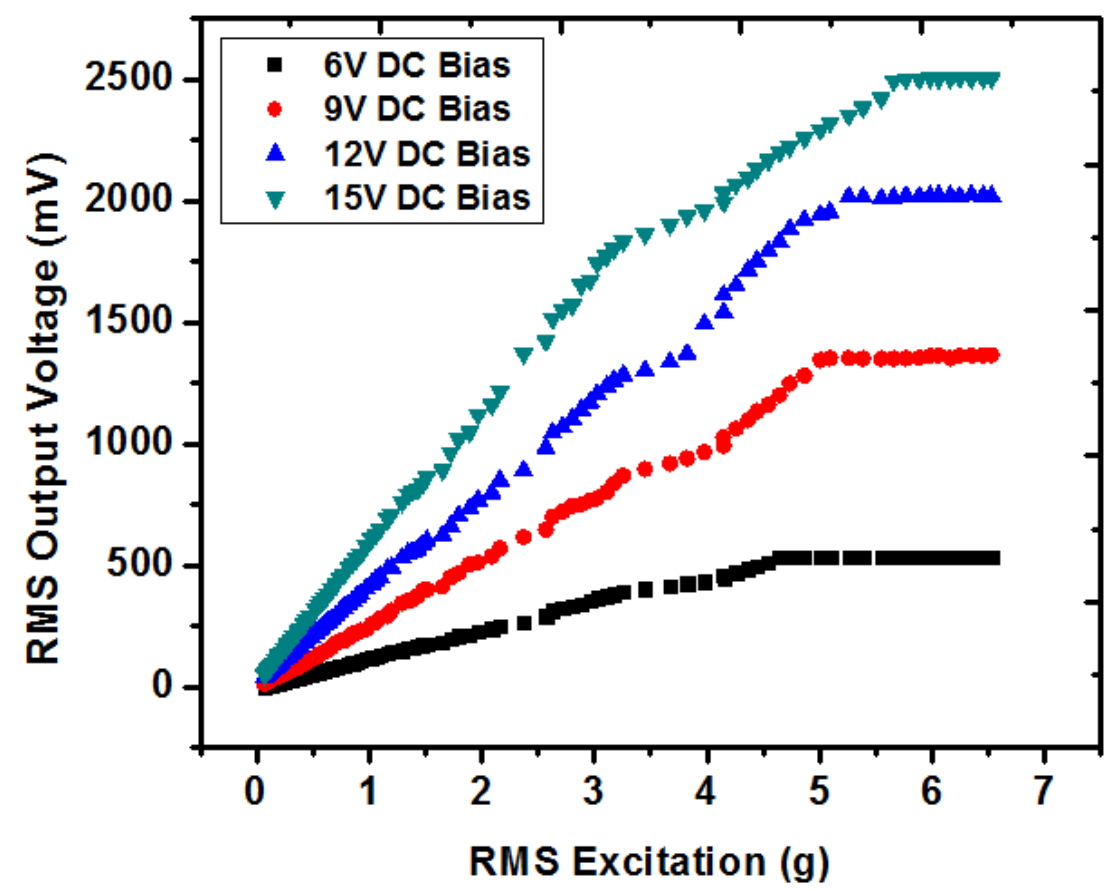

Figure 5.9. The RMS output voltage from bottom cavity of the devices with $30 \mu \mathrm{m}$ movable plate and $5 \mu \mathrm{m}$ suspension beams as excitation sweeps under a fixed frequency of $500 \mathrm{~Hz}$, (a) the RMS output voltage from single capacitor device as function of excitation acceleration, (b) the RMS output voltage from $1 \times 4$ capacitor array device as function of excitation acceleration. 
(a)

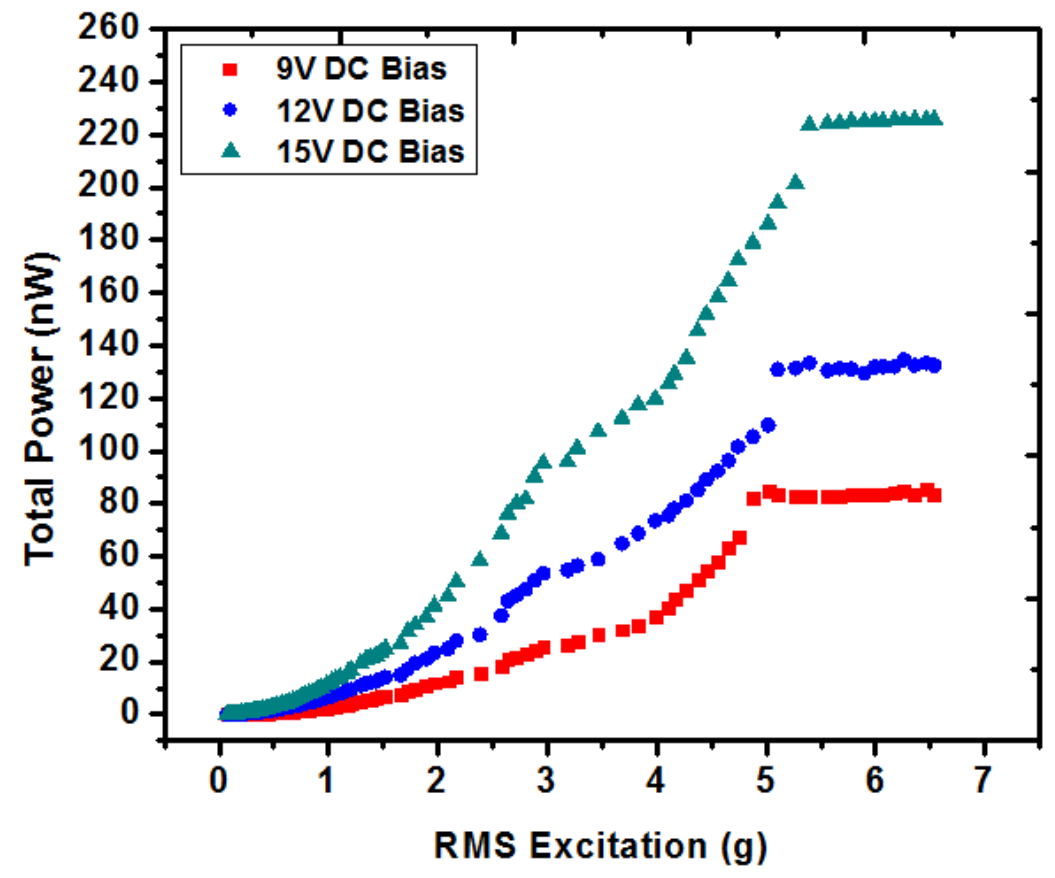

(b)

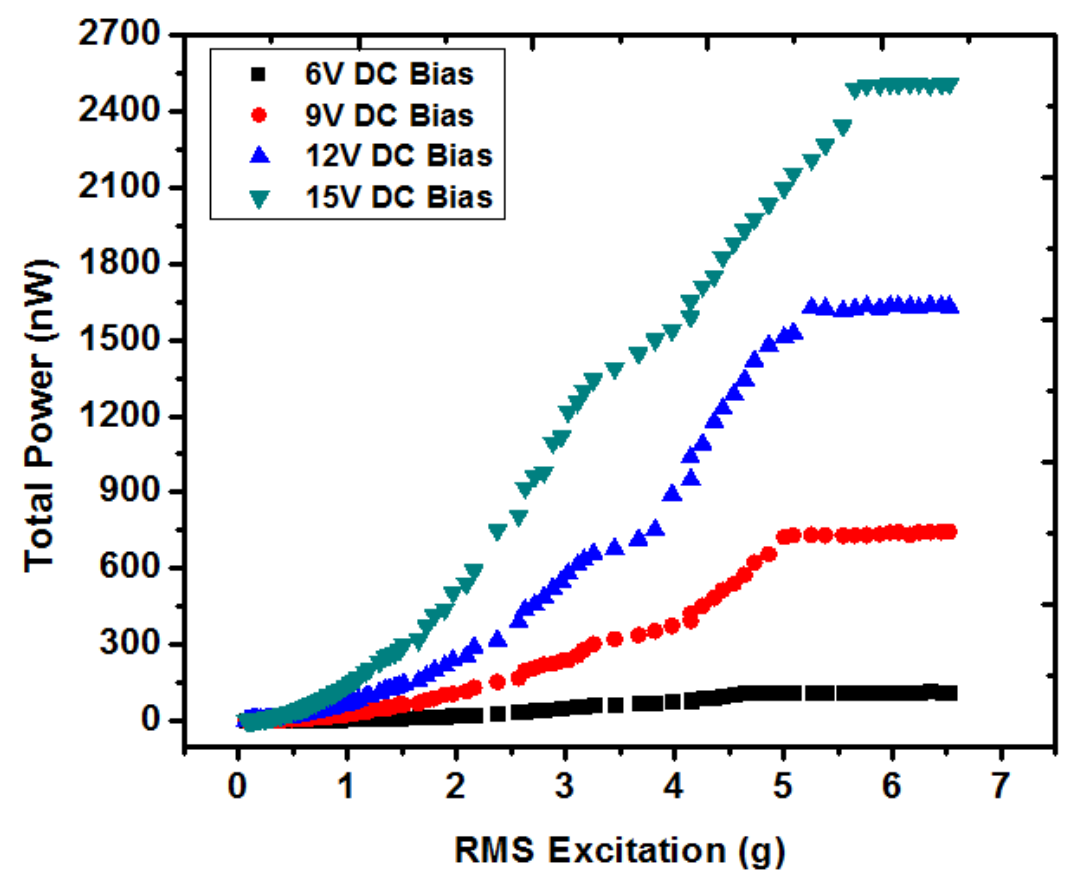

Figure 5.10. The total power across two load resistors from the devices with $30 \mu \mathrm{m}$ movable plate and $5 \mu \mathrm{m}$ suspension beams as excitation sweeps under a fixed frequency of $500 \mathrm{~Hz}$, (a) total power generated by single capacitor device as function of excitation acceleration, (b) total power generated by $1 \times 4$ capacitor array device as function of excitation acceleration. 
(a)

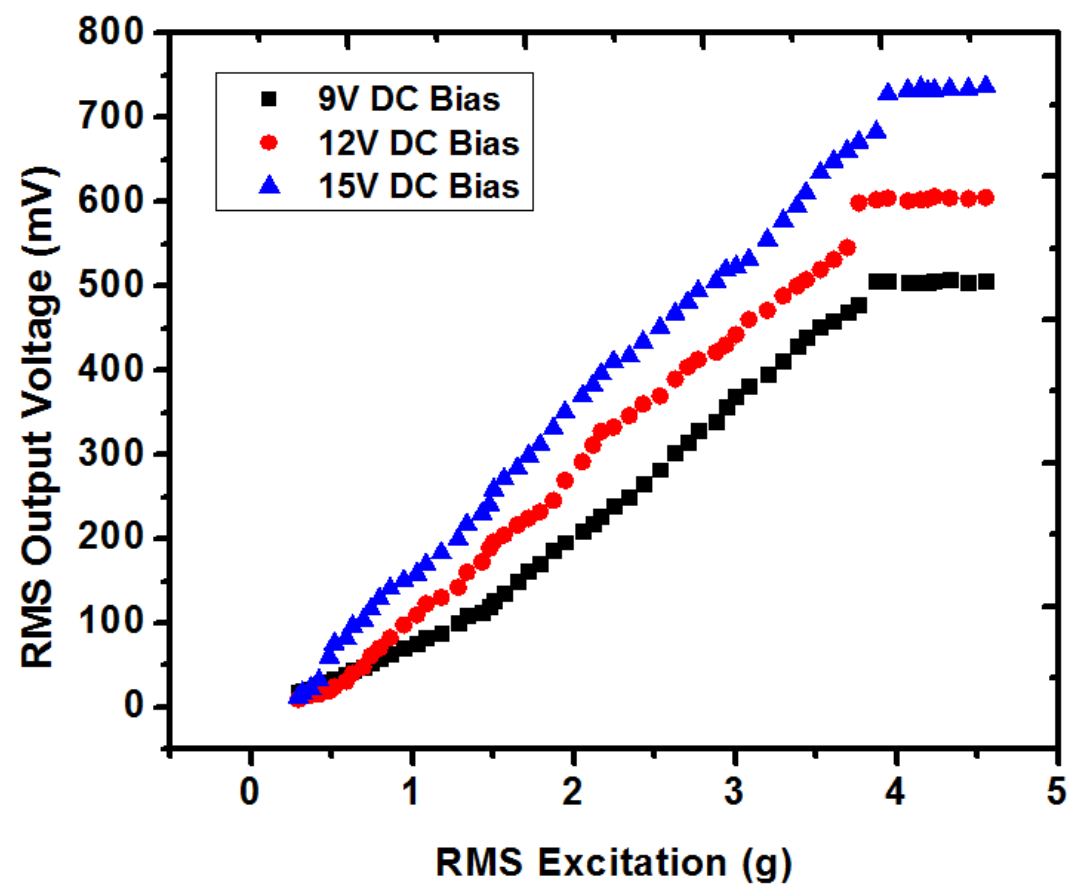

(b)

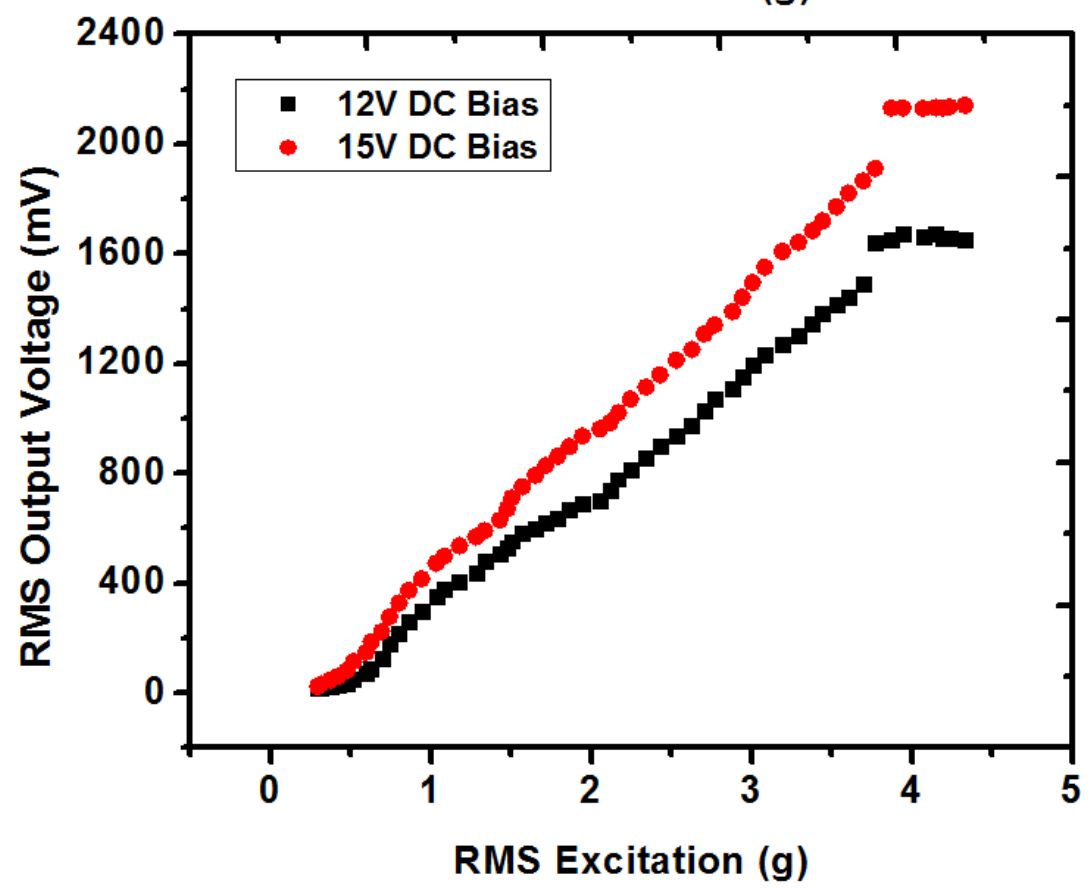

Figure 5.11. The RMS output voltage from bottom cavity of the devices with $40 \mu \mathrm{m}$ movable plate and $3 \mu \mathrm{m}$ suspension beams as excitation sweeps under a fixed frequency of $500 \mathrm{~Hz}$, (a) the RMS output voltage from single capacitor device as function of excitation acceleration, (b) the RMS output voltage from $1 \times 4$ capacitor array device as function of excitation acceleration. 
(a)

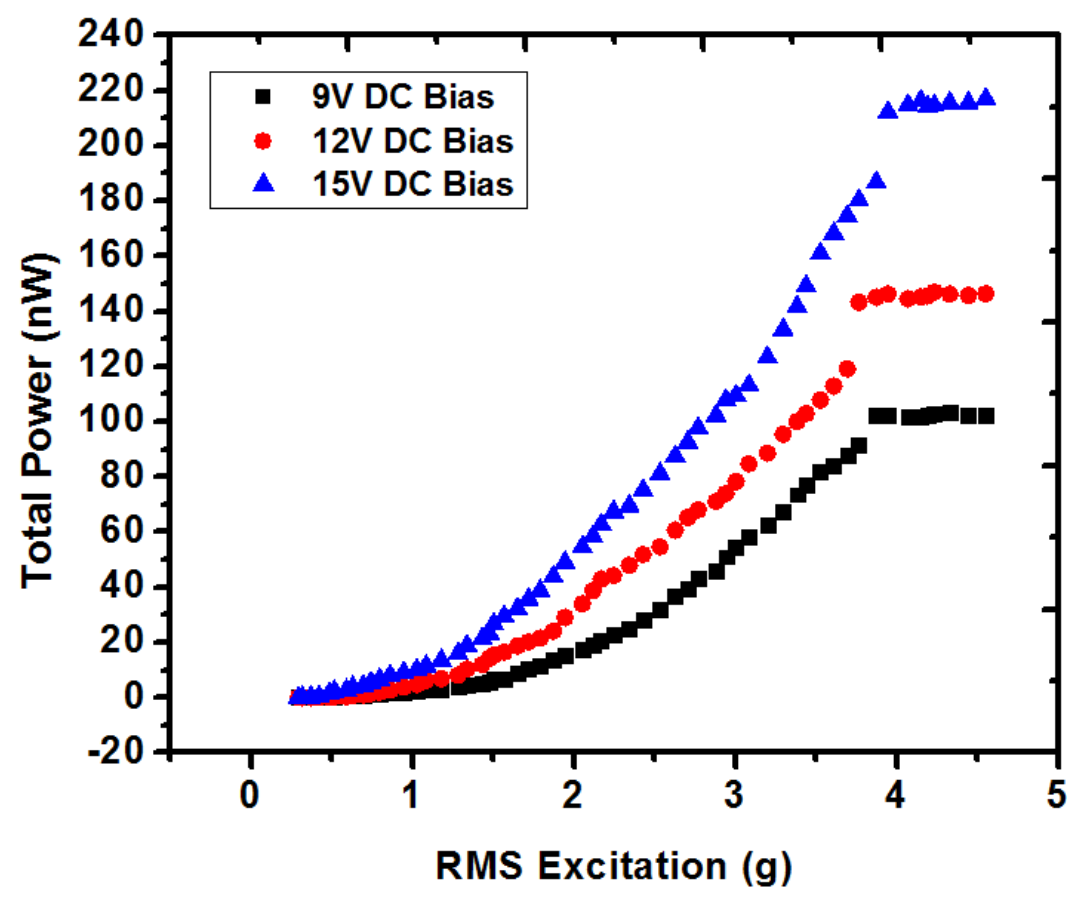

(b)

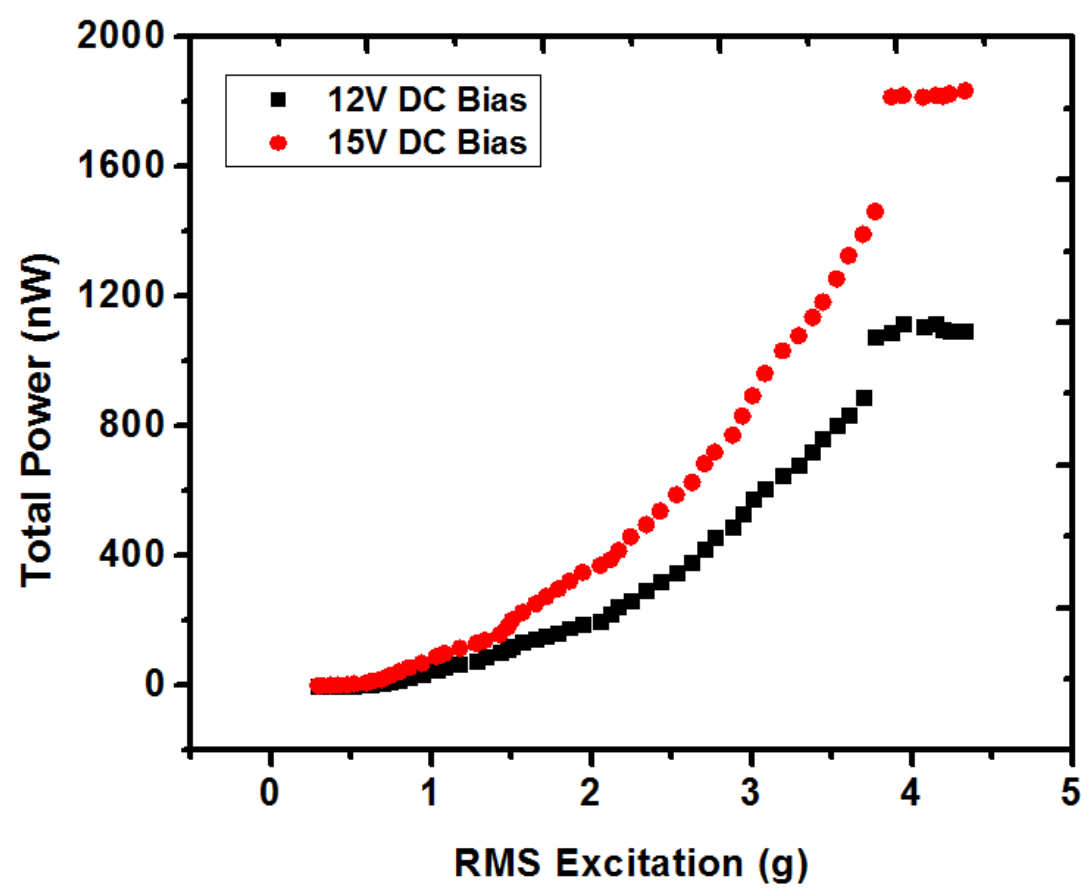

Figure 5.12. The total power across two load resistors from the devices with $40 \mu \mathrm{m}$ movable plate and $3 \mu \mathrm{m}$ suspension beams as excitation sweeps under a fixed frequency of $290 \mathrm{~Hz}$, (a) total power generated by single capacitor device as function of excitation acceleration, (b) total power generated by $1 \times 4$ capacitor array device as function of excitation acceleration. 
capacitor array achieved a maximum power around $216 \mathrm{nW}$ and $1.83 \mu \mathrm{W}$, respectively, under the high DC bias of $15 \mathrm{~V}$ and excitation above $3.7 \mathrm{~g}$ as shown in Figure 5.12(a) and 5.12(b). The capacitor array energy harvester generated 2.9 times of RMS output voltage in average than that of a single capacitor energy harvester based upon comprehensive testing results. All testing results of the fabricated devices with two different dimensions are summarized in Table 10 for comparison.

Table 10. Summary of the testing results of all fabricated devices.

\begin{tabular}{|c|c|c|c|c|}
\hline & \multicolumn{2}{|c|}{$\begin{array}{c}\text { MEMS energy harvester } \\
\text { single capacitor }\end{array}$} & \multicolumn{2}{c|}{$\begin{array}{c}\text { MEMS energy harvester } \\
1 \times 4 \text { capacitor array }\end{array}$} \\
\hline $\begin{array}{c}\text { Thickness of movable } \\
\text { plate }\end{array}$ & $30 \mu \mathrm{m}$ & $40 \mu \mathrm{m}$ & $30 \mu \mathrm{m}$ & $40 \mu \mathrm{m}$ \\
\hline $\begin{array}{c}\text { Thickness of } \\
\text { suspension beams }\end{array}$ & $5 \mu \mathrm{m}$ & $3 \mu \mathrm{m}$ & $5 \mu \mathrm{m}$ & $3 \mu \mathrm{m}$ \\
\hline $\begin{array}{c}\text { Resonance } \\
\text { Frequency }\end{array}$ & $500 \mathrm{~Hz}$ & $290 \mathrm{~Hz}$ & $500 \mathrm{~Hz}$ & $290 \mathrm{~Hz}$ \\
\hline $\begin{array}{c}\text { Maximum RMS } \\
\text { output voltage }\end{array}$ & $751 \mathrm{mV}$ & $736 \mathrm{mV}$ & $2505 \mathrm{mV}$ & $2141 \mathrm{mV}$ \\
\hline $\begin{array}{c}\text { Maximum } \\
\text { Power }\end{array}$ & $225 \mathrm{nW}$ & $216 \mathrm{nW}$ & $2.51 \mu \mathrm{W}$ & $1.83 \mu \mathrm{W}$ \\
\hline
\end{tabular}

\subsection{Frequency sweep testing with increasing excitation}

In an oscillating system, squeeze film air damping can make significant effects on the moving amplitude of the oscillating part and the resonance frequency, especially for those MEMS oscillators with parallel-plate structure [107, 108]. The stiffening effect of squeeze film air damping tends to increase the resonance frequency of the movable plate. On the other hand, when the movable plate is getting close to the fixed plate, the electro- 
(a)

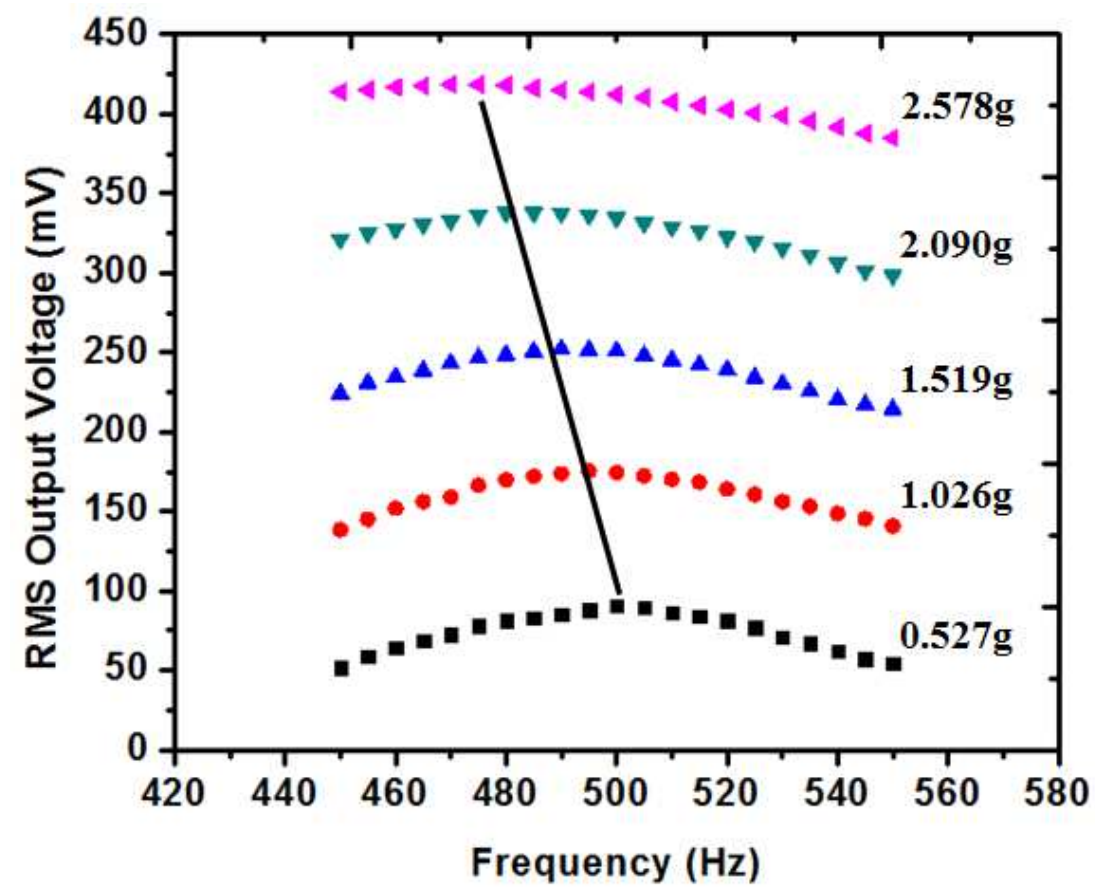

(b)

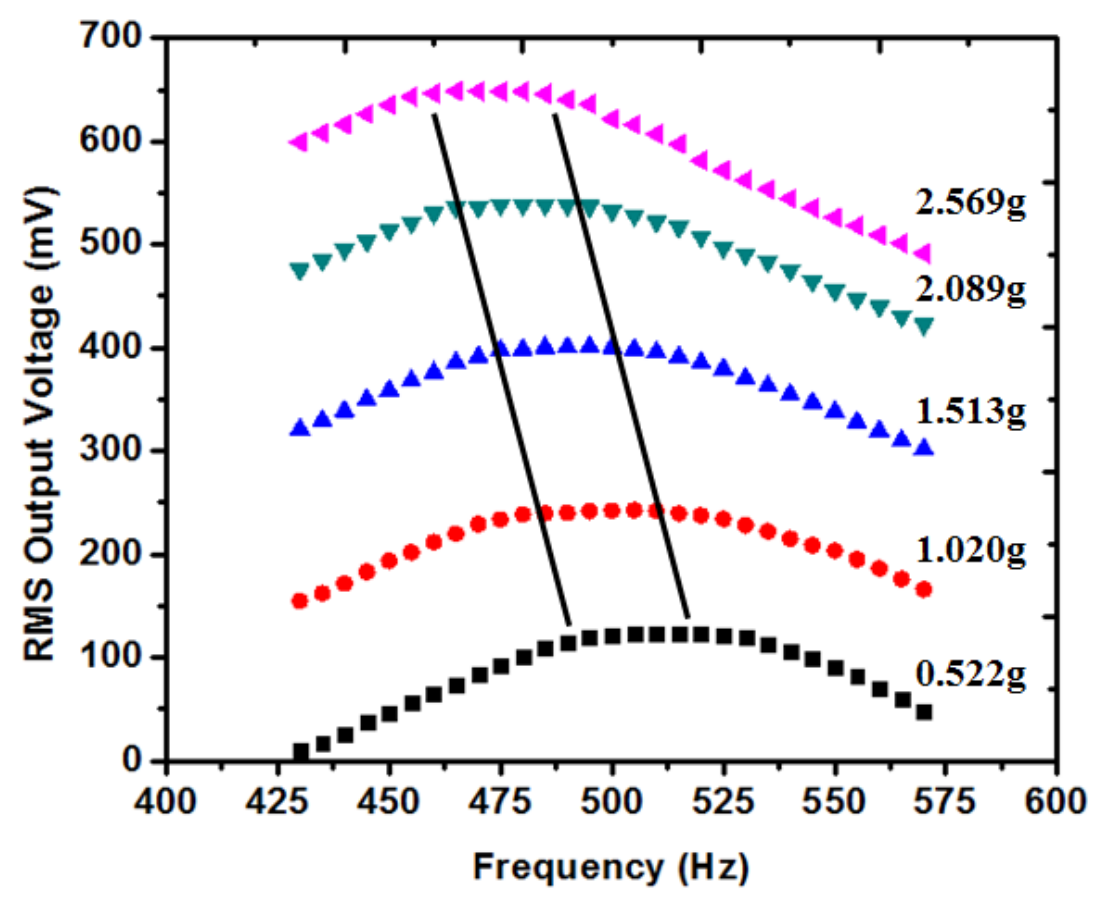

Figure 5.13. The RMS output voltage from bottom cavity of the devices as frequency sweeps under increasing excitation acceleration, (a) the RMS output voltage from single capacitor, (b) the RMS output voltage from $1 \times 4$ capacitor array. 
-static force between two plates is increasing nonlinearly. Therefore, the electrostatic force has softening effect in nature because it can reduce the resonance frequency determined by the elastic suspension. In this testing, we experimentally investigated the overall nonlinear effects for our MEMS variable capacitor by sweeping the excitation frequency within a narrow band width around devices' resonance frequency, and meanwhile increasing the excitation acceleration. In Figure 5.13(a), the RMS output voltage from bottom cavity of a single capacitor device with $30 \mu \mathrm{m}$ movable plate and 5 $\mu \mathrm{m}$ suspension beams under different excitation accelerations was plotted as function of frequency which was sweeping up from 450 to $550 \mathrm{~Hz}$ with steps of $5 \mathrm{~Hz}$. It is observed that the resonance frequency decreases from approximately 500 to $470 \mathrm{~Hz}$ as the excitation acceleration increases from 0.5 to $2.5 \mathrm{~g}$. The same experiment was implemented on the $1 \times 4$ capacitor array device with $30 \mu \mathrm{m}$ movable plate and $5 \mu \mathrm{m}$ suspension beams. The device was similarly excited as the frequency increased from 430 to $570 \mathrm{~Hz}$ under increasing excitation accelerations from 0.5 to $2.5 \mathrm{~g}$. The resonance frequency was also observed decreasing as the excitation was gradually increased as shown in Figure 5.13(b).

In this experiment, the observed resonance frequency shifting is an evident phenomenon reflecting the softening effect of suspension beams. The lowering of the resonance frequency can be considered as a result of the effect of softening induced by the nonlinear electrostatic force which is dominant over the effect of stiffening induced by the squeeze film air damping. However, the conjecture is drawn purely base upon experimental behaviors. We currently do not have a developed numerical program to evaluate the overall nonlinear effects mathematically because our system is a complicated 
nonlinear dynamics with perforated movable plate. Analytical analysis of squeeze film air damping for such MEMS device requires modified Reynolds equation involving the damping effect of gas flow through holes on the movable plate [109]. 


\section{CHAPTER 6}

\section{CONCLUSION}

A novel MEMS variable capacitor with two-cavity has been designed and fabricated for energy harvesting utilizing unwanted mechanical vibration in environment. The device is unique in the use of two capacitive cavity structure and nickel (Ni) as the main structural material. Two designs of MEMS variable capacitor have been proposed including a single MEMS variable capacitor and MEMS variable capacitor array.

The proposed designs have been drawn and modeled using L-edit MEMS CAD tool and COMSOL Multi-Physics finite element tool. The designed structure was confirmed with good mechanical durability and appropriate resonance response. Numerical programs have been developed to simulate the dynamic motion of the movable plate and calculate the total harvested power for given vibration amplitude and frequencies using Matlab. The two-cavity design was proved with higher average power than conventional single-cavity design under a wide range of vibration frequencies and amplitudes based on the simulation results. Rocking instability, i.e. perturbations that may result in movable plates not in parallel to fixed plates, of the movable plate in our two-cavity structure has also been studied base upon comprehensive dynamic simulations using Matlab. The results show that the resultant symmetric breaking can cause drastic reduction on power generation.

A single MEMS variable capacitor and $1 \times 4$ capacitor array with two-cavity for energy harvesting from environmental vibration has been successfully fabricated using surface micromachining technology. Extensive characterizations of the fabricated devices 
have been performed using a PCB test board mounted on a shaker connected with a data acquisition board (NI USB-6218 DAQ). Both kinds of the devices were observed with a resonance frequency around $500 \mathrm{~Hz}$. However, the resonance frequency of the device with capacitor array achieved broader bandwidth due to slightly different resonance response from each capacitor. When the excitation acceleration was increased, a maximum power of $2.5 \mu \mathrm{W}$ across the load resistors was achieved from the MEMS energy harvester using $1 \times 4$ capacitor array under a high DC bias of $15 \mathrm{~V}$ and intense excitation amplitude above $5 \mathrm{~g}$. In comparison, the single capacitor device was reported with $220 \mathrm{nW}$ as the maximum output power. The comprehensive experimental results show that RMS voltage from the $1 \times 4$ capacitor array is 3.2 times in average of that from single capacitor. Therefore, we are able to draw the conclusion that the MEMS capacitor array is capable of generating 3.2 times higher power in average than the single capacitor.

In a second fabricated devices with modified dimensions, a single MEMS variable capacitor and $1 \times 4$ capacitor array with heavier movable proof mass plate and less stiff serpentine suspension beams were also developed for lower frequency application. In this case, the movable proof mass plate thickness was increased to $40 \mu \mathrm{m}$ while the thickness of suspension beams was decreased to $3 \mu \mathrm{m}$. The testing results demonstrate a lower resonance frequency of $290 \mathrm{~Hz}$, and the measured total power across the load resistors from single capacitor device and capacitor array device achieved a maximum power around $216 \mathrm{nW}$ and $2.14 \mu \mathrm{W}$, respectively, under a high DC bias of $15 \mathrm{~V}$ and extremely intense excitation above $3.7 \mathrm{~g}$.

The nonlinear effects of squeezed film air damping, electrostatic force and elastic force of suspension was experimentally investigated. The overall nonlinear effects for the 
two designs of MEMS variable capacitors with $30 \mu \mathrm{m}$ thick movable plate and $5 \mu \mathrm{m}$ thick suspension beams was studied by sweeping the excitation frequency within a narrow band width around devices' resonance frequency, meanwhile, gradually increasing the excitation acceleration. It is observed that the resonance frequency of the single capacitor device decreases from approximately 500 to $470 \mathrm{~Hz}$ as the excitation acceleration increases from 0.5 to $2.5 \mathrm{~g}$. A similar experiment was implemented on the 1 $\times 4$ capacitor array device. The resonance frequency of the $1 \times 4$ capacitor array device was also observed decreasing as the excitation was gradually increased. The observed resonance frequency lowering can be considered as a result of that the effect of softening induced by the nonlinear electrostatic force is dominant over the effect of stiffening induced by the squeeze film air damping, however, the conjecture is drawn purely base upon experimental behaviors. 


\section{REFERENCES}

[1] Prime Faraday Technology Watch, "An introduction to MEMS," Loughborough University, UK: PRIME Faraday Partnership, ISBN 1-84402-020-7, 2002

[2] J. M. Bustillo, R. T. Howe and R. S. Muller, "Surface micromachining for microelectromechanical systems," Proceedings of the IEEE, vol. 86, no. 8, 1998.

[3] S. K. George and B. Koc, "Free-form simulation of sequential etching and surface characterization for 3-D MEMS fabrication," Journal of Microelectromechnical Systems, vol. 18, no. 2, pp. 296-307, 2009.

[4] J. N. Helbert, "Handbook of VLSI Microlithography," 2nd Edition, 2011, pp 1-5

[5] Y. K. Yoon, J. H. Park, and M. G. Allen, "Multidirectional UV lithography for complex 3-D MEMS structures," Journal of Microelectromechnical Systems, vol. 15, no. 5, pp. 1121-1130, 2006.

[6] G. C. Schwartz and P. M. Schaible, "Reactive ion etching of silicon," Journal of Vacuum Science and Technology, vol 16, pp 410-413,1979.

[7] F. Marty, L. Rousseau, B. Saadany, B. Mercier, O. Français, Y. Mita and T. Bourouina, "Advanced etching of silicon based on deep reactive ion etching for silicon high aspect ratio microstructures and three-dimensional micro- and nanostructures," MicroElectronics Journal, vol 36, pp 673-677, 2005

[8] Q. Chen, J. Fang, H. F. Ji and K. Varahramyan, "Fabrication of $\mathrm{SiO}_{2}$ microcantilever using isotropic etching With ICP," IEEE Sensor Journal, vol. 17, no. 2, 2007.

[9] Y. T. Yang, T. K. Won, S. Y. Choi, T. Takehara, Y. Nishimura and J. M. White, "The latest plasma-enhanced chemical-vapor deposition technology for large-size processing," Journal of Display Technology, vol. 3, no. 4, 2007.

[10] M. A. George, H. Chandra, P. Morse and J. Madocks, "Latest innovations in large area web coating technology via plasma enhanced chemical vapor deposition source technology," Journal of Vacuum Science and Technology A: Vacuum, Surfaces, and Films, vol. 27, no. 4, 2009.

[11] R. B. Fair, "History of some early developments in ion-implantation technology leading to silicon transistor manufacturing," Proceedings of the IEEE, vol. 86, no. $1,1998$. 
[12] A. J. Chung, Y. S. Huh and D. Erickson, "A robust, electrochemically driven microwell drug delivery system for controlled vasopressin releasell," Biomedical Microdevices, vol. 11, pp 861-867, 2009.

[13] M. Du, X. Y. Ye, K. Wu and Z.Y. Zhou, "A peristaltic micro pump driven by a rotating motor with magnetically attracted steel balls," Sensors, vol 9, pp 26112620,2009

[14] M. Shoji and I. Hiroyuki, "Optically driven viscous micropump using a rotating microdisk," Applied Physics Letters, vol. 91, pp 084101-084101, 2007.

[15] T. W. Lim, Y. Son, Y. J. Jeong, D. Y. Yang, H. J. Kong, K. S. Lee and D. P. Kim, "Threedimensionally crossing manifold micro-mixer for fast mixing in a short channel length," Lab on a Chip, vol. 11, pp 100-103, 2011.

[16] P. I. Oden, P. G.Datskos, T. Thundat and R. J.Warmack, "Uncooled thermal imaging using a piezoresistive microcantilever," Applied Physics Letters, vol. 69, pp 3277-3279, 1996.

[17] D. W. Dareing, T. Thundat, S. Jeon and M. Nicholson, "Modal analysis of microcantilever sensors with environmental damping," Journal of Applied Physics, vol. 97, pp. 084902-084902, 2005.

[18] W. C. Qu, S. K. Islam, M. R. Mahfouz, M. R. Haider, G. To and S. Mostafa, "Microcantilever array pressure measurement system for bomedical instrumentation,” IEEE Sensors Journal, vol. 10, pp. 321-330, 2010.

[19] G. Meng, W. M. Zhang, H. Huang, H. G. Li and D. Che, "Micro-rotor dynamics for microelectro-mechanical systems (MEMS)," Chaos, Solitons and Fractals, vol. 40, pp 538-562, 2009.

[20] M. Godin, A. K. Bryan, T. P. Burg, K. Babcock and S. R. Manalis, "Measuring the mass, density, and size of particles and cells using a suspended microchannel resonator," Applied Physics Letters, vol. 91, 2007.

[21] V. Maselli, R. Osellame, G. Cerullo, R. Ramponi, P. Laporta, L. Magagnin and P. L. Cavallotti, "Fabrication of long microchannels with circular cross section using astigmatically shaped femtosecond laser pulses and chemical etching," Applied Physics Letters, vol. 88, 2006.

[22] T. Lemke, J. Kloeker, G. Biancuzzi, T. Huesgen, F. Goldschmidtboeing and P. Woias, "Fabrication of a normally-closed microvalve utilizing lithographically 
defined silicone micro O-rings," Journal of Micromechanics and Microengineering, vol. 21, no. 025011, 2011.

[23] Y. Wu, J. D. Benson, J. K. Critser and M. Almasri, "MEMS-based coulter counter for cell counting and sizing using multiple electrodes," Journal of Micromechanics and Microengineering, vol. 20, no. 085035, 2010.

[24] Q. Cheng, Z. Sun, G. A. Meininger and M. Almasri, "Mechanical study of micromachined polydimethylsiloxane elastic microposts," Review of Scientific Instruments, vol. 81(10) 106104, 2010.

[25] Q. Cheng, S. Paradis, T. Bui and M. Almasri, "Design of dual-band uncooled infrared microbolometer,” IEEE Sensors Journal, vol.11, no.1, pp. 167-175, 2011.

[26] R. J. M. Vullers, R. van Schaijk, I. Doms, C. Van Hoof and R. Mertens, "Micropower energy harvesting," Solid-State Electronics, vol. 53, pp. 684-693, 2009.

[27] C. He, M. E. Kiziroglou, D. C. Yates and E. M. Yeatman, "A MEMS self-powered sensor and RF transmission platform for WSN nodes," IEEE Sensor Journal, vol. 11 , no. $12,2011$.

[28] S. Roundy, P. K. Wright and J. Rabaey, "A study of low level vibrations as a power source for wireless sensor nodes," Computer Communications, vol. 26, no. 11, pp. 1131-1144, 2003.

[29] E. M. Yeatman, "Energy scavenging for wireless sensor nodes," 2nd International Workshop on Advances in Sensors and Interface, pp. 1 - 4, 2007.

[30] S. Bermejo, S. Silvestre, P. Ortega and G. Herrera, "Light harvesting photovoltaic mini-generator," Progress in Photovoltaics: Research and Applications, vol. 20, no.8, 2012.

[31] P. Ortega, S. Bermejo and L. Castaner, "High voltage photovoltaic mini-modules," Progress in Photovoltaics: Research and Applications, vol. 16, no.5, 2008.

[32] M. A. Green, "Third generation photovoltaics: advanced solar energy conversion," Springer Verlag, 2004.

[33] M. Kishi, H. Nemoto, T. Hamao, M. Yamamoto, S. Sudou, M. Mandai and S. Yamamoto, "Micro thermoelectric modules and their application to wristwatches as an energy source," 18th International Conference on Thermoelectrics, pp. 301307, 1999. 
[34] Z. Y. Wang, V. Leonova, P. Fiorini and C. V. Hoof, "Realization of a wearable miniaturized thermoelectric generator for human body applications," Sensors and Actuators A, vol. 156, no.1, 2009.

[35] U. Bergqvist et al., "Mobile telecommunication base stations: exposure to electromagnetic fields," Report of a short term mission within COST-244bis, COST-244bis short term mission on base station exposure, 2000.

[36] H. J. Visser, A. C. F. Reniers and J. A. C. Theeuwes, "Ambient RF energy harvesting: GSM and WLAN power density measurements," European microwave conference, Amsterdam, the Netherlands, 2008.

[37] R. J. M. Vullers, H. J. Visser, B. Op het Veld and V. Pop, "RF harvesting using antenna structures on foil," Proceedings of PowerMEMS 2008, pp. 209-212, Sendai, Japan, 2008.

[38] http://www.powercastco.com

[39] C. B. Williams and R. B. Yates, "Analysis of a micro-electric generator for microsystems," Sensors and Actuators A, vol. 52, pp. 8-11, 1996.

[40] T. J. Kazmierski and S. Beeby, "Energy Harvesting Systems - Principles, Modeling and Applications," Springer Science+Business Media, LLC, New York, 2011.

[41] P.D. Mitcheson, T.C. Green, E.M. Yeatman and A.S. Holmes, "Architectures for Vibration driven Micropower," Journal of Microelectromechnical Systems., vol. 13, no. 3, 2004.

[42] C. He, M. E. Kiziroglou, D. C. Yates and E. M. Yeatman, "A MEMS self-powered sensor and RF transmission platform for wsn nodes," IEEE Sensor Journal, vol. 11, no. 12, 2011.

[43] J. F. M. Oudenhoven, R. J. M. Vullers and R. Schaijk, "A review of the present situation and future developments of micro-batteries for wireless autonomous sensor systems," International Journal of Energy Research, vol. 36, issue 12, pp. 1139-1150, 2012.

[44] R. Torah, P. Glynne-Jones, M. Tudor, T. O'Donnell, S. Roy and S. Beeby, "Selfpowered autonomous wireless sensor node using vibration energy harvesting," Measurement Science and Technology, vol. 19, no. 12, p. 125 202, 2008. 
[45] N. G. Elvin, N. Lajnef and A. A. Elvin, "Feasibility of structural monitoring with vibration powered sensors," Smart Materials and Structures, vol. 15, no. 4, pp. 977-986, 2006.

[46] E. R. Westby and E. Halvorsen, "Design and modeling of a patterned-electretbased energy harvester for tire pressure monitoring systems," IEEE/ASME Transactions on Mechatronics, vol. 17, no. 5, 2012.

[47] F. Herrault, B. C. Yen, C. H. Ji, Z. S. Spakovszky, J. H. Lang and M. G. Allen, "Fabrication and performance of silicon-embedded permanent-magnet microgenerators," Journal of Microelectromechanical Systems, vol. 19, no. 1, pp. 4-13, 2010.

[48] E. Jovanov and A. Milenkovic, "Body area networks for ubiquitous healthcare applications: opportunities and challenges," Journal of Medical Systems, vol. 35, no. 5, pp. 1245-1254, 2011.

[49] A. Shamim, M. Arsalan, L. Roy, M. Shams, and G. Tarr, "Wireless dosimeter: System-on-chip versus system-in-package for biomedical and space applications," IEEE Transactions on Circuits and Systems II, vol. 55, no. 7, pp. 643-647, 2008.

[50] Y. Suzuki1, D. Miki, M. Edamoto and M. Honzumi, "A MEMS electret generator with electrostatic levitation for vibration-driven energy-harvesting applications," Journal of Micromechanics and Microengineering, vol. 20, no. 104002, 2010.

[51] R. J. M. Vullers, R. V. Schaijk, H. J. Visser, J. Penders and C. V. Hoof, "Energy harvesting for autonomous wireless sensor networks," IEEE Solid-State Circuits Magazine, vol. 2, no. 2, pp. 29-38, 2010.

[52] F. Cheli, E. Leo, S. Melzi and E. Sabbioni, "On the impact of 'smart tyres' on existing ABS/EBD control systems," Vehicle System Dynamics, vol. 48, no. S1, pp. 255-270, 2010.

[53] M. Löhndorf, T. Kvisterøy, E. Westby and E. Halvorsen, "Evaluation of energy harvesting concepts for tire pressure monitoring systems," Proceedings of Power MEMS 2007, pp. 331-334, Freiburg, Germany, 2007.

[54] R. Elfrink, S. Matova, C. de Nooijer, M. Jambunathan, M. Goedbloed, J. van de Molengraft, V. Pop, R. J. M. Vullers, M. Renaud and R. van Schaijk, "Shock induced energy harvesting with a MEMS harvester for automotive applications," Proceedings of IEEE/IEDM, pp. 677-680, 2011. 
[55] T. Sterken, P. Fiorini, G. Altena, C. Van Hoof and R. Puers, "Harvesting energy from vibrations by a micromachined electret generator," Proceedings of Transducers Conference, pp. 129-132. 2007.

[56] D. A. Wang, C. Y. Chiu and H. T. Pham, "Electromagnetic energy harvesting from vibrations induced by Kármán vortex street," Mechatronics, vol. 22, no. 6, pp. 746-756, 2012.

[57] P. Wang, X. Dai, X. Zhao and G. Ding, "A micro electromagnetic vibration energy harvester with sandwiched structure and air channel for high energy conversion efficiency," Proceedings of PowerMEMS 2009, pp. 296-299, Washington DC, U.S.A., 2009.

[58] S. P. Beeby, R. N. Torah, M. J. Tudor, P. Glynne-Jones, T. ODonnell, C. R. Saha and S. Roy, "A micro electromagnetic generator for vibration energy harvesting," Journal of Micromechanics and Microengineering, vol. 17, no. 071257, 2007.

[59] K. Ashraf, M. H. M. Khir, J. O. Dennis and Z. Baharudin, "A wideband, frequency up-converting bounded vibration energy harvester for a low-frequency environment," Smart Materials and Structures, vol. 22, no. 2, p. 025018, 2013.

[60] J. A. Paradiso and T. Starner, "Energy scavenging for mobile and wireless electronics," IEEE Pervasive Computing, vol.145-146, pp. 336-342, 2008.

[61] A. Khaligh, P. Zeng and C. Zheng, "Kinetic energy harvesting using piezoelectric and electromagnetic technologies-State of the art," IEEE Transactions on Industrial Electronics, vol. 57, no. 3, pp. 850-860, 2010.

[62] S. R. Anton and H. A. Sodano, "A review of power harvesting using piezoelectric materials (2003-2006)," Smart Materials and Structures, vol. 16, no. 3 pp. R1R21, 2007.

[63] R. Xu, A. Lei, T.L. Christiansen, K. Hansen, M. Guizzetti, K. Birkelund, E.V. Thomsen and O. Hansen, "Screen printed PZT/PZT thick film bimorph MEMS cantilever device for vibration energy harvesting," Proceedings of IEEE Tranducer'11, pp. 679-682, Beijing, China, 2011.

[64] A. Massaro, S. De Guido, I. Ingrosso, R. Cingolani, M. De Vittorio, M. Cori, A. Bertacchini, L. Larcher and A. Passaseo, "Freestanding piezoelectric rings for high efficiency energy harvesting at low frequency," Applied Physics Letters, vol. 98, no. 5, pp. 053502-053502-3, 2011. 
[65] S. Xu, K. D. T. Ngo, T. Nishida, G. B. Chung and A. Sharma, "Low frequency pulsed resonant converter for energy harvesting," IEEE Transactions on Power Electronics, vol. 22, no. 1, pp. 63-68, Jan. 2007.

[66] C. Xu, B. Ren, W. Di, Z. Liang, J. Jiao, "Cantilever driving low frequency piezoelectric energy harvester using single crystal material $0.71 \mathrm{~Pb}(\mathrm{Mg} 1 / 3 \mathrm{Nb} 2 / 3) \mathrm{O}_{3}-0.29 \mathrm{PbTiO}_{3}$," Applied Physics Letters, vol. 101, issue 3, p. 083502, 2012.

[67] D. F. Berdy, P. Srisungsitthisunti, B. Jung, X. Xu, J. F. Rhoads and D. Peroulis, "Low-frequency meandering piezoelectric vibration energy harvester," IEEE Transactions on Ultrasonics, Ferroelectrics and Frequency Control, vol. 59, issue 5, 2012.

[68] H. Liu, C. J. Tay, C. Quan, T. Kobayashi and C. Lee, "Piezoelectric MEMS energy harvester for low-frequency vibrations with wideband operation range and steadily increased output power," Journal of Microelectromechanical Systems, vol. 20, no. 5, pp. 1131-1142, 2011.

[69] A. Hajati and S. G. Kim, "Ultra-wide bandwidth piezoelectric energy harvesting," Applied Physics Letters, vol. 99, no. 8, pp. 083105-083105-3, 2011.

[70] S. Meninger, J. O. Mur-Miranda, R. Amirtharajah, A. P. Chandrakasan and J. H. Lang, "Vibration-to-electric energy conversion," IEEE Transactions on VLSI Systems, vol. 9, no. 1, pp. 64-76, 2001.

[71] S. Roundy, P. Wright and K. Pister, "Micro-electrostatic vibration-to-electricity converters," Proceedings of IMECE, pp. 1-10, 2002.

[72] S. D. Nguyen and E. Halvorsen, "Nonlinear springs for bandwidth-tolerant vibration energy harvesting," Journal of Microelectromechanical Systems, vol. 20, no. 6, pp. 1225-1227, 2011.

[73] L. G. W. Tvedt, D. S. Nguyen and E. Halvorsen, "Nonlinear behavior of an electrostatic energy harvester under wide-and narrowband excitation," Journal of Microelectromechanical Systems, vol. 19, no. 2, pp. 305-316, 2010.

[74] D. Hoffmann, B. Folkmer and Y. Manoli, "Fabrication, characterization and modelling of electrostatic micro-generators," Journal of Micromechanics and Microengineering, vol. 19, no. 094001, 2009. 
[75] E. O. Torres and G. A. Rincón-Mora, "Electrostatic energy-harvesting and batterycharging CMOS system prototype," IEEE Transactions on Circuits and Systems, vol. 56, no. 9, pp. 1938-1948, 2009.

[76] B. Andò, S. Baglio, G. L'Episcopo and C. Trigona, "Investigation on mechanically bistable MEMS devices for energy harvesting from vibrations," Journal of Microelectromechanical Systems, vol.21, no.4, pp. 779-790, 2012.

[77] P. Basset, D. Galayko, A. M. Paracha, F. Marty, A. Dudka and T. Bourouina, "A batch-fabricated and electret-free silicon electrostatic vibration energy harvester," Journal of Micromechanics and Microengineering, vol. 19, no. 115025, 2009.

[78] W. Ma, R. Q. Zhu, L. Rufer, Y. Zohar and M. Wong, "An integrated floatingelectrode electric microgenerator," Journal of Microelectromechanical Systems, vol.16, no. 1, pp. 29-37, 2007.

[79] Yong Zhu, S. O. Reza Moheimani and Mehmet Rasit Yuce, "A 2-DOF MEMS ultrasonic energy harvester," IEEE Sensor Journal, vol. 11, no. 1, 2011.

[80] S. D. Nguyen, E. Halvorsen and I. Paprotny, "Bistable springs for wideband microelectromechanical energy harvesters," Applied Physics Letters, vol. 102, 2013.

[81] P. D. Mitcheson, P. Miao, B. H. Stark, E. M. Yeatman, A. S. Holmes and T. C. Green, "MEMS electrostatic micropower generator for low frequency operation," Sensors and Actuators A: Physical, vol. 115, issue 2-3, pp. 523-529, 2004.

[82] P. Miao, P. D. Mitcheson, A. S. Holmes, E. M. Yeatman, T. C. Green and B. H. Stark, "MEMS inertial power generators for biomedical applications," Microsystem Technologies, vol. 12, Issue 10-11, pp. 1079-1083, 2006.

[83] M. Kranza, M.G. Allen and T. Hudson, "In situ wafer-level polarization of electret films in MEMS acoustic sensor arrays," Sensors and Actuators A: Physical, vol. 188, pp. 181-189, 2012

[84] Y. Arakawa, Y. Suzuki and Kasagi, "Micro seismic power generator using electret polymer film," Proceedings of PowerMEMS 2004, pp. 187-190, Kyoto, Japan, 2004.

[85] W. H. Hsieh, T. Y. Hsu and Y. C. Tai, "A Micromachined thin-film teflon electret microphone,” IEEE Transducer’97, pp. 425-428, Chicago, U.S.A., 1997. 
[86] K. Matsumoto, K. Saruwatari and Y. Suzuki, "Vibration-powered battery-less sensor node using MEMS electret generator," Proceedings of PowerMEMS 2011, pp. 134-137, Seoul, Korean, 2011.

[87] Y. Minakawa and Y. Suzuki "Low-resonant-frequency MEMS electret energy harvester with $\mathrm{x}$-shaped high-aspect-ratio parylene spring," Proceedings of PowerMEMS 2012, pp. 133-136, Atlanta, U.S.A., 2012.

[88] R. Chen and Y. Suzuki, "Metal-on-nothing electrodes for reducing parasitic capacitance in electret energy harvesting devices," Proceedings of PowerMEMS 2012, pp. 121-124, 2012.

[89] D. S. Nguyen, E. Halvorsen, G. U. Jensen and A. Vogl, "Fabrication and characterization of a wideband MEMS energy harvester utilizing nonlinear springs," Journal of Micromechanics and Microengineering, vol. 20, no. 125009, 2010.

[90] C. P. Le, E. Halvorsen, O. Søråsen and E. M. Yeatman, "Microscale electrostatic energy harvester using internal impacts," Journal of Intelligent Material Systems and Structures, vol. 23, no. 13, pp. 1409-1421, 2012.

[91] C. P. Le and E. Halvorsen, "MEMS electrostatic energy harvesters with end-stop effects," Journal of Micromechanics and Microengineering, vol. 22, no. 074013, 2012.

[92] Z. Stanimirović and I. Stanimirović, "Mechanical properties of MEMS materials," Micro Electronic and Mechanical Systems, chapter 11, pp. 572, INTECH, Croatia, ISBN 978-953-307-027-8, 2009.

[93] I. Sari, T. Balkan and H. Kulah, "An electromagnetic micro power generator for wideband environmental vibrations," Sensors and Actuators A: Physical, vol. 145146, pp.405-413, 2008.

[94] S. M. Shahruz, "Design of mechanical band-pass filters for energy scavenging," Journal of Sound and Vibration, vol. 292, pp.3-5, 2006.

[95] T. Petropoulos, E. M. Yeatman and P. D. Mitcheson, "MEMS coupled resonators for power generation and sensing," Micromechanics Europe, pp. 261-264, 2004.

[96] S. Roundy, "On the effectiveness of vibration-based energy harvesting," Journal of Intelligent Material Systems and Structures, vol. 16, no. 10, pp 809-823, 2005. 
[97] J. Lin, J. X. Zhu, M. Sonje, Y. S. Chang, Z. C. Feng, and M. Almasri, “Two-cavity MEMS Variable Capacitor for Power Harvesting," Journal of Micromechanics and Microengineering, vol. 22, no. 065003, 2012.

[98] J. F. Rhoads, S. W. Shaw and K. L. Turner, "Nonlinear dynamics and its applications in micro- and nanoresonators," Journal of Dynamic Systems, Measurement, and Control, vol. 132, no. 034001-2, 2010.

[99] E. K. Chan, K. Garikipati and R. W. Dutton, "Characterization of contact electromechanics through capacitance-voltage measurements and simulations," Journal of Microelectromechanical Systems, vol. 8, no. 2, pp. 208-217, 1999.

[100] P. B. Chu and K. S. J. Pister, "Analysis of closed-loop control of parallel plate electrostatic microgrippers," Proceedings of IEEE Robotics and Automation, pp. 820-825, 1994.

[101] D. Hoffmann, B. Folkmer and Y. Manoli, "Fabrication, characterization and modelling of electrostatic micro-generators," Journal of Micromechanics and Microengineering, vol. 19, no. 094001, 2009.

[102] J. X. Zhu, Z. C. Feng, J. Lin, N. Yusek and M. Almasri, "Dynamic study of MEMS variable capacitive device for power harvesting," Proceedings of ASME International IDETC and CIE, 2012.

[103] J. Lin, J. X. Zhu, Y. S. Chang, Z. C. Feng, and M. Almasri, "Surface micromachined MEMS capacitors with dual-cavity for energy harvesting," Journal of Microelectromechanical Systems, vol. pp, issue 99, 2013.

[104] W. J. Daughton and F. L. Givens, "An investigation of the thickness variation of spun-on thin films commonly associated with the semiconductor industry," Journal of The Electrochemical Society, vol. 129, p. 173, 1982.

[105] M. A. Spak, "High temperature post exposure bake for AZ 4000 photoresist," SPIE, vol. 539, p. 299, 1985.

[106] H. H. Lou and Y. Huang, "Encyclopedia of chemical processing," DOI: 10.1081/E-ECHP-120007747

[107] L. Mol, L. A. Rocha, E. Cretu and R. F. Wolffenbuttel, "Squeezed film damping measurements on a parallel-plate MEMS in the free molecule regime," Journal of Micromechanics and Microengineering, vol. 19, no. 074021, 2009. 
[108] M. H. Bao and H. Yang, "Squeeze film air damping in MEMS," Sensors and Actuators A: Physical, vol. 136, issue 1, 2007.

[109] M. H. Bao, H. Yang, Y.C. Sun and P. J. French, "Modified Reynolds' equation and analytical analysis of squeeze-film air damping of perforated structures," Journal of Micromechanics and Microengineering, vol. 13, no. 06-795, 2003. 


\section{LIST OF PUBLICATIONS}

\section{Journals:}

J. Lin, J. X. Zhu, Y. S. Chang, Z. C. Feng, and M. Almasri, "Surface Micromachined MEMS Capacitors with Dual-Cavity for Energy Harvesting," IEEE/ASME Journal of Microelectromechanical Systems, vol. 22, issue 6, Dec. 2013.

J. Lin, J. X. Zhu, M. Sonje, Y. S. Chang, Z. C. Feng, and M. Almasri, "Two-cavity MEMS Variable Capacitor for Power Harvesting", Journal of Micromechanics and Microengineering, vol. 22, no. 6, Mar. 2012.

\section{Conferences:}

J. Lin, J. X. Zhu, Z. C. Feng, and M. Almasri, "MEMS Electrostatic Power Harvesters Array with Two-Cavity”, IEEE Transducer 2013, abstract accepted, 2013.

J. Lin, J. X. Zhu, Z. C. Feng, and M. Almasri, "Electrostatic Micro Power Scavenger with Dual Cavities", Proc. PowerMEMS, Atlanta, U.S.A., Dec. 2012.

J.X. Zhu, Z.C. Feng, J. Lin, N. Yusek and M. Almasri, "Dynamic Study of MEMS Variable Capacitive Device for Power Harvesting", Proc. ASME International IDETC and CIE, 2012.

J. Lin, J. X. Zhu, M. Sonje, Z. C. Feng, M. Almasri, "Two-cavity MEMS capacitive power scavenger", Proc. SPIE Defense, Security and Sensing 2012, 8377-25, Apr. 2012.

J. Lin, J. X. Zhu, M. Sonje, N. Yusek, Z. C. Feng, and M. Almasri, "Multi-cavities MEMS Capacitive Plates for Power Harvesting", Proc. PowerMEMS, Seoul, Korean, Nov. 2011.

J. Lin, M. Sonje, S. Nanduri, N. Yusek, Z. C. Feng, and M. Almasri, "Fabrication and Modeling Analysis of the MEMS Capacitive Plates with Large Dynamic Range for Voltage Conversion and Power Harvesting", Proc. NSF Engineering Research and Innovation Conference, Atlanta, Georgia, Jan. 2011.

\section{Patent disclosure:}

J. Lin, N. Yusek, Z. Feng, M. Almasri, "MEMS Multi-Cavity Capacitive Power Harvester", U.S. Patent disclosure, in process, 2013. 
Jie Lin was born on April 20th, 1986 in Putian, Fujian province, China. He received the B.S. degree with double majors in Physics and Electronic Engineering from Pittsburg State University (PSU), Pittsburg, KS, in 2009, and received the Ph.D. degree in Electrical and Computer Engineering at the University of Missouri (MU), Columbia, MO, in 2013.

He was a research assistant with the Kansas Polymer Research Center at PSU working on polymer engineering and characterization. Since August 2009, he had been a graduate research assistant with the Microsystem Research Lab in the Department of Electrical and Computer Engineering at MU, focusing on his doctoral research in the area of MEMS. His research interests include electrostatic MEMS energy harvesting, 3D BioMEMS sensor for foodborne pathogens detection, uncooled microbolometers for infrared detection, and microfabrication process development. He is currently joining the Intel Cooperation as Process Technology Development Engineer focusing on semiconductor manufacturing and process technology development. 